\title{
An exploration of salutogenic factors promoting workforce health and well-being in small and medium-sized businesses in East-Westphalia Lippe/Germany
}

\section{Julia Anna Pönighaus}

\author{
A thesis submitted to \\ the University of Gloucestershire \\ in accordance with the requirements of the degree of \\ Doctor of Business Administration (DBA) \\ in the Faculty of Business and Technology
}

UNIVERSITY OF

GLOUCESTERSHIRE

September, 2019 


\section{Abstract}

This thesis explores the research gap in salutogenic factors promoting workforce health and well-being generated by top management of small and medium-sized enterprises (SMEs) in East-Westphalia Lippe (EWL), in Germany.

The workplace health promotion (WHP) is, and will be, a relevant area of research because the growing absenteeism, the reduced work performance and the increased turnover rates in businesses are believed to be some of the major challenges of the 21st-century. It is well documented that WHP has many advantages for SMEs but only a few recognise the necessity of WHP.

The thesis chooses a new way in WHP in SMEs while using salutogenesis as a theoretical framework in order to give the thesis a special focus on employees' well-being. In addition, salutogenesis is successfully used in various professional fields for example medicine and nursing. But there is a research gap in implementing salutogenesis in SMEs.

The originality consists of connecting the salutogenesis (SoC model) with a current study about health promoting factors by Bruch et al. (2013) which were used as salutogenic factors and tested for usability in SMEs. Consequently, the thesis implements the theoretical premise of salutogenesis to SMEs and explores how, or to what extent, applicable the concept of salutogenesis is in SMEs in EWL.

The primary question is: What salutogenic factors can be generated by top management to support the promotion of health and well-being of the workforce? The second research question meets the important role of the top manager as the decision maker of WHP: How can SMEs develop top management awareness to keep their staff healthy? The third key research question is: How can SMEs in EWL/Germany integrate health promoting and well-being factors? 
The research methodology is based on the literature review and the empirical research based on focus- group discussions of top managers and employees of SMEs in EWL. A critical realist prejudice was developed as a foundation for an Applied Thematic Analysis (ATA). A reflection group was used to compare the authors' interpretation.

\section{The core findings are:}

The findings from the small-scale research suggest: The salutogenic factors can be mainly found in the quality of the relationship of top managers with their employees.

The thesis delivers the new findings to WHP research: 'the importance of the quality of the relationship', and the 'importance of the capacity of older top managers to reflect'. The latter is at the same time the surprising finding of this thesis. Whether the higher age of top managers has significant influences on WHP has to be explored in future research.

For salutogenic WHP in SMEs, it seems to be optimal for the top managers to act in a relationship-oriented sense with the capacity to reflect themselves.

SMEs that implement salutogenic factors are headed in the right direction to be fit for the future, by taking into account the needs of employees in the sense of salutogenesis and thus keeping their most important resource, the workforce, healthy.

Further additional research is needed about experiences of implementation of the SoC in SMEs.

\section{Key words}

Salutogenesis, Sense of Coherence $(\mathrm{SoC})$, health and well-being of employees, workplace health promotion (WHP), corporate health management (CHM), top management, and SMEs. 


\section{Author's declaration}

I declare that the work in this thesis was carried out in accordance with the regulations of the University of Gloucestershire and is original except where indicated by specific reference in the text. No part of the thesis has been submitted as part of any other academic award. The thesis has not been presented to any other education institution in the United Kingdom or overseas. Any views expressed in the thesis are those of the author and in no way represent those of the university.

Signed:

Date: 23.09.2019 


\section{Acknowledgements}

First of all, I wish to thank my dear parents who give me a lot of love, supporting me and believing in me all the time. Especially, I want to thank my father, who has taught me never to give up. Many thanks to my mother for the many uplifting conversations and the everlasting interest in my thesis topic. I would particularly like to thank the most important person in my life, my son Maximilian. When I look into his eyes, I know who I am doing this project for. Thank you for sustaining me with love, Maximilian.

In my family there is another important person whom I would like to thank from my heart, my Uncle Peter. Only through your support could I fulfil my dream of a doctoral thesis. Thank you so much! I would also like to thank my godmother Margret, who is always there when I need her and who also supported me during this time.

A very special thanks goes to my supervisors, without them this thesis would have never been finished, Dr. Francisca Veale and Dr. Sören Petter whose support and comments have contributed enormously to this research and my own development - it was a pleasure to work with both of you.

Many thanks to the participants who participated in my research, for their time and openness in the group interviews without which my research would not have been possible.

This research was supported by my employer, the University of Applied Sciences (Fachhochschule des Mittelstands (FHM)) with its CEO and rector Prof. Dr. Anne Dreier, who enabled me the best conditions for research. Many thanks especially to Prof. Dr. Volker Wittberg, Prof. Dr. Philippa Ward and Dr. Metje Rocklage, who have always supported and encouraged me. I also thank Lawrie Kerr who has trained me in English and has taken over the English correction of my thesis. 
"The miracle

I have been seeking

for so long,

It is actually myself."

(Selma Lagerlöf) 


\section{Table of contents}

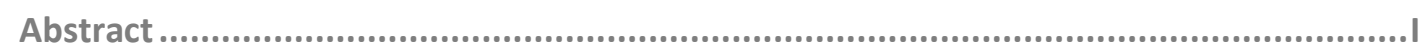

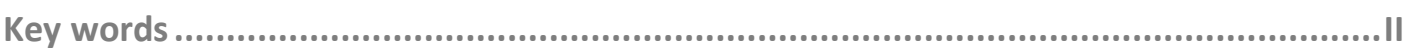

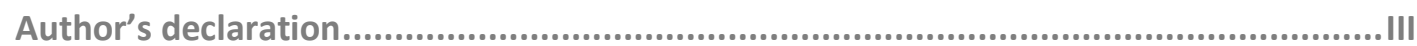

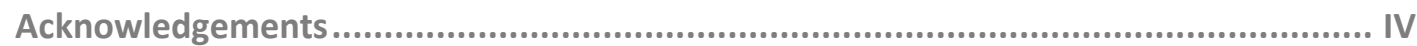

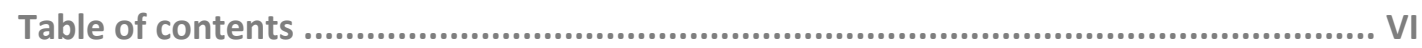

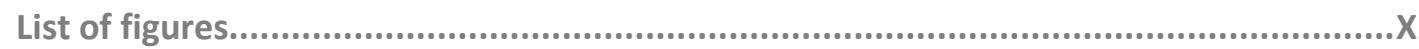

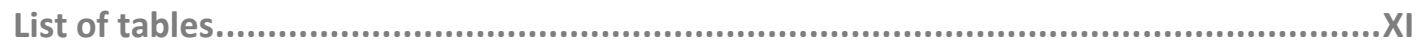

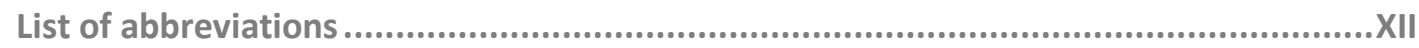

1. Introduction

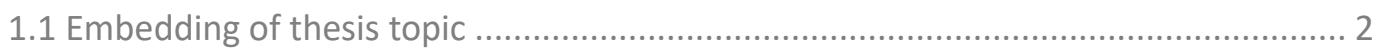

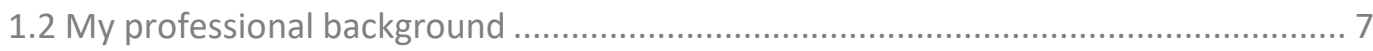

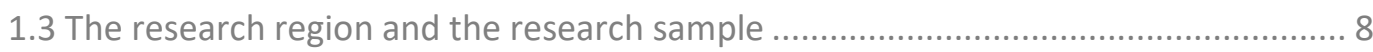

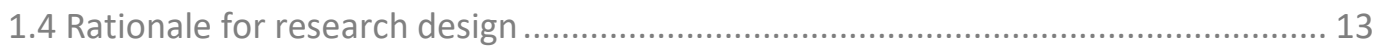

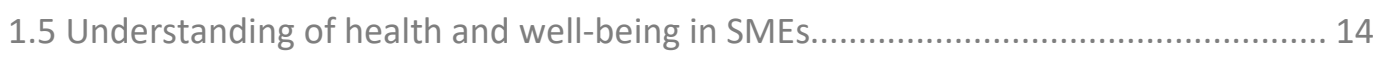

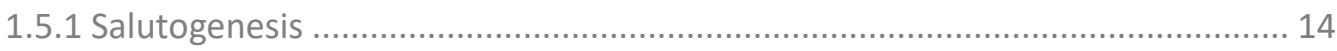

1.5.2 Research study about developing a healthy performance culture in SMEs by

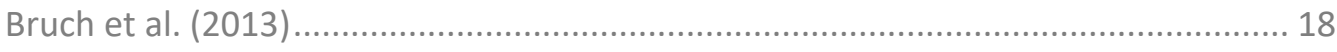

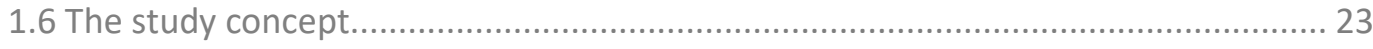

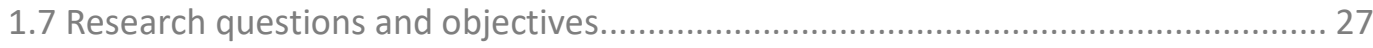

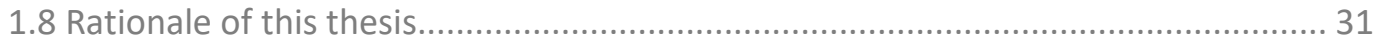

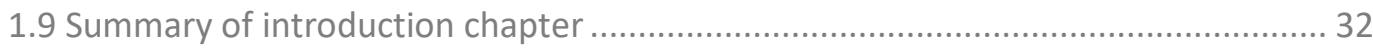

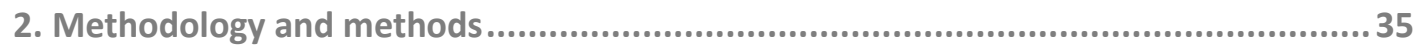

2.1 Study aim with its inclusion and exclusion criteria.................................................. 36

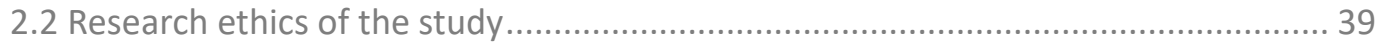

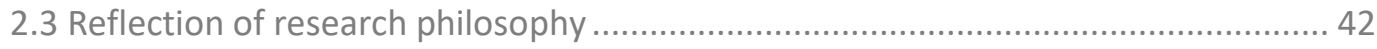

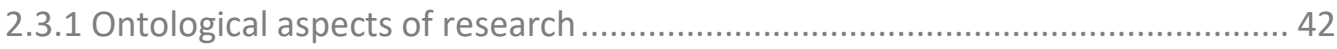

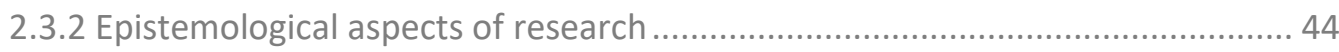

2.3.3 The use of critical realist paradigm for this study........................................... 47

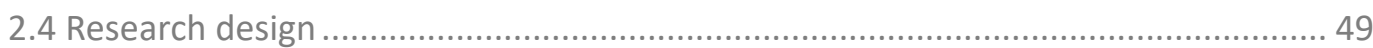

Julia Anna Pönighaus $\quad$ VI 


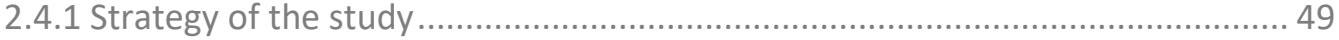

2.4.2 Qualitative character of the study design ........................................................ 50

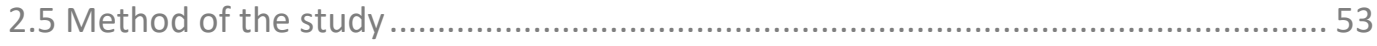

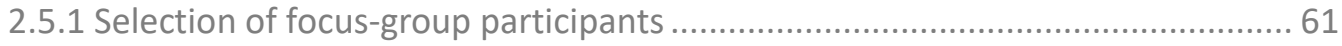

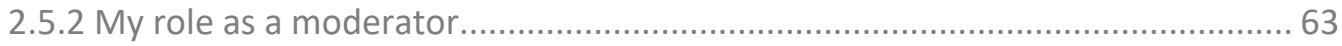

2.5.3 Duration and size of focus-group discussions ..................................................... 64

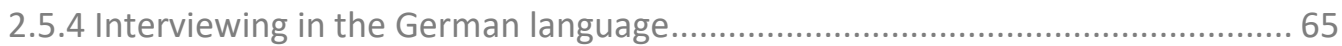

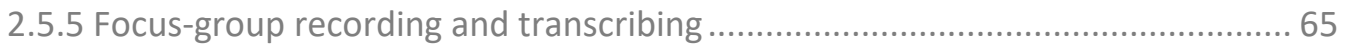

2.5.6 Consideration regarding reliability and validity ............................................... 66

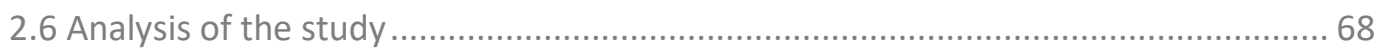

2.6.1 Applied Thematic Analysis selected for focus-group discussions analysis ......... 68

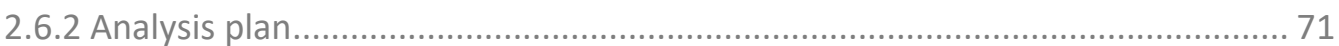

2.6.3 Process for extracting codes and themes from focus groups........................... 73

2.6.4 Qualitative Data Analysis Software - NVivo ...................................................... 75

2.7 Summary of methodology and method chapter .................................................... 76

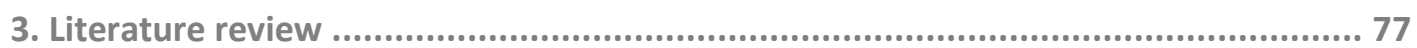

3.1 Origin and development of systematic literature review technique ........................ 77

3.2 Methodology of systematic literature review …………………............................... 79

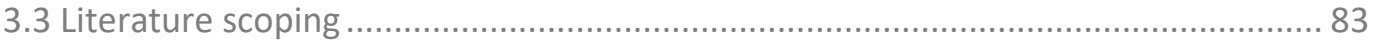

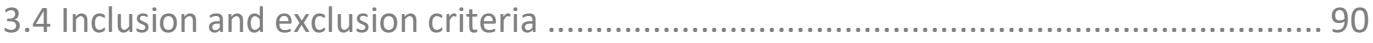

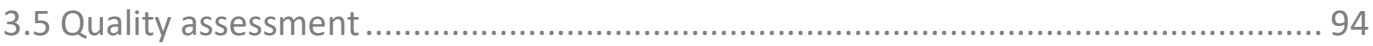

3.6 Integrating and synthesising qualitative research................................................... 100

3.6.1 Rationale of choosing synthesis method ...................................................... 100

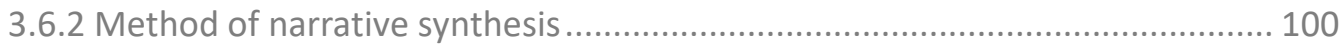

3.6.3 Synthesising qualitative research on health and well-being promotion of

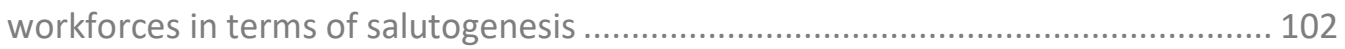

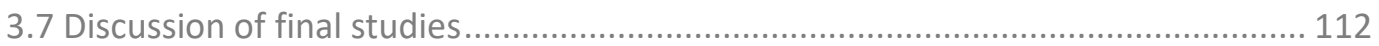

3.8 Review findings for answering the research questions ........................................ 114

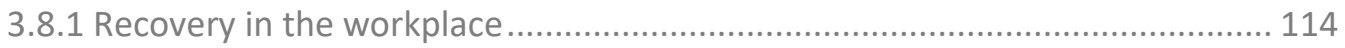

3.8.2 Providing a resourceful working environment ............................................... 115

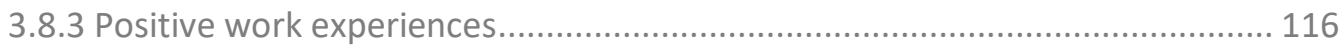

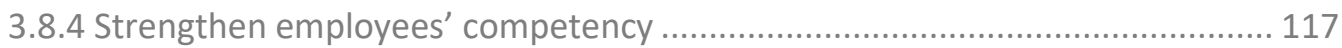

3.8.5 Usefulness of the SoC theory in workplace health promotion ......................... 117 
3.8.6 Leadership

4. Findings of the focus-group discussions using the Applied Thematic Analysis

4.1 Overview of general information about focus-group members 125

4.2 Reviewing and defining potential themes 125

4.2.1 Summary and presentation of themes with their contribution to SoC and the factors of the study by Bruch et al. (2013)....

4.3. Implementing a reflection focus group discussion....

4.3.1 Analyses and overview of findings of the reflection focus group..... 144

4.3.2 Summary and presentation of themes of the reflection group with their contribution to SoC and the factor of the study by Bruch et al. (2013)

4.4 Study findings which answer the study research questions.... 150

4.4.1 Research Question 1: 150

4.4.2 Research Question 2: 152

4.4.3 Research Question 3: 153

4.5 Summary of study findings

\section{Discussion of study findings in the context of review findings and study research} questions

6. Implications for further research

7. Conclusions of salutogenic WHP research in SMEs

7.1 Relevance of research

7.2 Implications and limitations of research findings.......................................... 176

7.2.1 Qualitative research design 176

7.2.2 Research answer 1: Exploration of salutogenic factors supporting the promotion of health and well-being of workforces....

7.2.3 Research answer 2: Exploration of how SMEs can develop top management awareness to keep their staff healthy

7.2.4 Research answer 3: Understanding of how SMEs in East-Westphalia

Lippe/Germany integrate health promoting and well-being factors....

7.2.5 Limitations of research study 180

7.2.6 Surprising outcome of this study 181

7.2.7 Contributions to knowledge 
7.2.8 Implications for theory, research and practice 183

7.3 Recommendations for further research and practice 186

7.4 Final remarks. 187

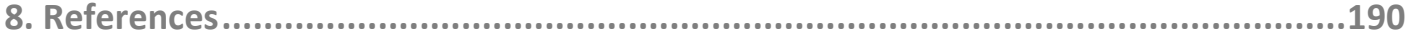

9. Appendix - table of content 199

9.1 Nine recommendations for health promotion within the study by Bruch et al. (2013) (translated by a translation agency). 200

9.2 Original of the guideline of the study by Bruch et al. (2013)... 204

9.3 Studies which met the quality assessment criteria. 205

9.4 Final studies with information after the first synthesis step 211

9.5 Final studies with information after the second synthesis step 213

9.6 Example of code table 217

9.7 Grading system assessing the robustness of the synthesis. 218

9.8 Example of 'Participant Information Sheet' (PIS) ................................................ 219

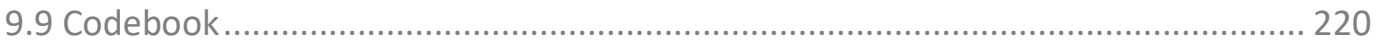

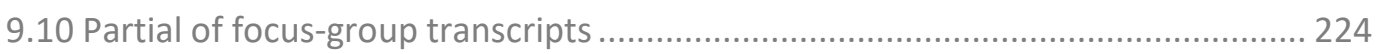

9.10.1 Focus-group discussion top managers ............................................................ 224

9.10.2 Focus-group discussion employees.............................................................. 226

9.10.3 Reflection focus-group discussion ............................................................. 228

9.11 Slides of the focus-group discussions ................................................................... 230

9.12 Summary of focus-group discussion data for the reflection group........................ 244 


\section{List of figures}

Figure 1: Health, stress and coping (Antonovsky, 1979).

Figure 2: Allocation of Antonovsky's SoC elements with the health promotion initiatives of the study by Bruch et al. (2013). .27

Figure 3: Structure of the three research questions (own figure) . ..............................................28

Figure 4: The critical realist research position (own figure).

Figure 5: The slide of the Power Point Presentation of focus-group discussion to introduce 'Ben

Müller'

Figure 6: Slide of the Power Point Presentation of focus-group discussion to introduce the topic 'top management as a pioneer'.

Figure 7: Example slide to introduce the kind of questions after representing each topic.

Figure 8: Slide of the Power Point Presentation of focus-group discussion to present the first open question.

Figure 9: Slide of the Power Point Presentation of focus-group discussion to present the second open question

Figure 10: Qualitative and Quantitative Data Analysis (adapted from Bernard, 1996). .68

Figure 11: Six phase process of thematic analysis (own figure, content adapted from Braun \& Clarke (2006)) .70

Figure 12: The coding process with basic terms in textual qualitative analysis (own figure)...........73

Figure 13: Screenshot of English terms.

Figure 14: Appraisal system of the quality assessment criteria and the topic foci..... .97

Figure 15: Phases of the process of thematic analysis (own figure, content adapted from Braun \& Clarke (2006)).

Figure 16: Overview of outstanding topics. 


\section{List of tables}

Table 1: Nine topics of the guideline (short version) within the study 'Healthy Leadership' (Bruch et

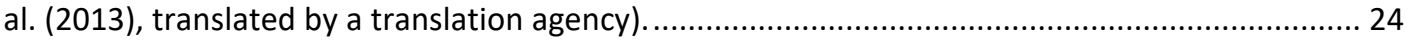

Table 2: Connection of research questions and research objectives.............................................. 30

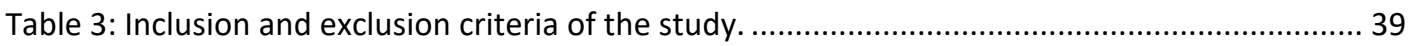

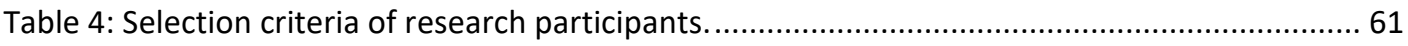

Table 5: Information about members of focus-group discussions............................................. 62

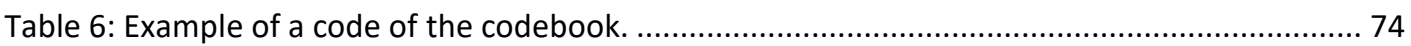

Table 7: Established NVivo project for this research project. ....................................................... 76

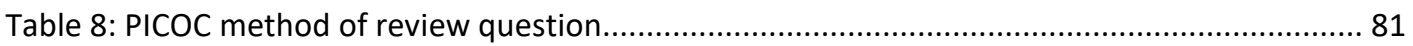

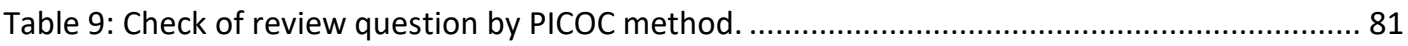

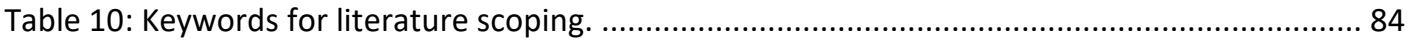

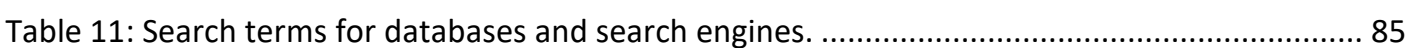

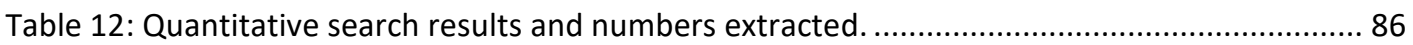

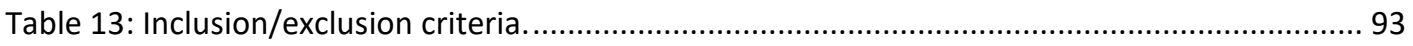

Table 14: Sources of the studies meeting the inclusion criteria.................................................. 94

Table 15: Quality assessment criteria, based on EPPI-Centre criteria (Shepherd et al., 2006)........ 96

Table 16: Studies which meet the quality assessment criteria. ..................................................... 97

Table 17: Example of table of the study of Ejlertsson et al. (2018)........................................... 108

Table 18: Tree of themes within this research project (a total of 11 participants)....................... 126

Table 19: Themes checked regarding relevance. ................................................................. 128

Table 20: Presentation of themes interpreted by me and by the reflection group....................... 145

Table 21: Themes of reflection group checked regarding relevance. ............................................ 146

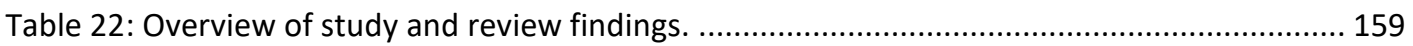

Table 23: Research questions and some research findings.................................................. 175 


\section{List of abbreviations}

ATA: Applied Thematic Analysis

BGM: Betriebliches Gesundheitsmanagement

CEO: Chief Executive Officer

CEBMa: Center for Evidence Based Management

CHM: Corporate Health Management

CRD: Centre for Reviews and Dissemination

DBA: Doctor of Business Administration

EWL: East-Westphalia Lippe

EPPI-Centre: The Evidence for Policy and Practice Information and Co-ordinating Centre

FGD: Focus-group discussion

FHM: Fachhochschule des Mittelstands

IFM: Institut für Mittelstandsforschung Bonn

IHK: Chamber of Commerce and Industry

JD-R model: Job-demands-resources model

OHM: Occupational Health Management

NHS: National Health Service (UK)

NVivo: Software package for qualitative data analysis

PICOC: Population, Intervention, Comparison, Outcome, Context

PwC: Pricewaterhouse Coopers

QDAS: Qualitative data analysis software

SLR: Systematic literature review

SMBs: Small and medium-sized businesses

SMEs: Small and medium-sized enterprises

SoC: Sense of Coherence

WHO: World Health Organization

WHP: Workplace health promotion 


\section{Introduction}

This practice-orientated 'Doctor of Business Administration' (DBA) thesis explores how small and medium-sized enterprises (SMEs) in East-Westphalia Lippe (EWL) in Germany can promote the health and well-being of their workforces. The aim of my study is to strengthen SMEs in EWL/Germany to meet their challenges by creating business conditions to keep their employees healthy. Therefore, it is important that health promotion activities of companies meet not only the physical side of health but also the mental health of workforces as many studies show (Badura et al., 2017; Matyssek, 2012; The National Association of Statutory Health Insurance Funds, 2017). In order to do justice to the importance of promoting mental health of employees, my study uses the synonymous term 'well-being' in addition to the term 'health'. Therewith, my study does justice to the current trend in health promotion focussing on the mental side of employees' health and understands health as a phenomenon that should be considered comprehensively. This holistic understanding of health and the comprehensive consideration of health as a unity of body and soul corresponds well with Antonovsky's salutogenesis and its 'Sense of Coherence' (SoC) model which is used as a theoretical framework for this study. Of course, there are other models or theories of health, but none is as influential as salutogenesis in health promotion (Franzkowiak et al., 2011). Moreover, as an interdisciplinary and integrative model salutogenesis is well suited to integrate it in different areas (Wydler et al., 2010). Therefore, it offers my study an ideal theoretical framework for investigating health and well-being promotion of workforces in SMEs in EWL/Germany.

Salutogenesis is already being considered in the social, medical and health sectors but not yet in business (Zimber, 2011). So, my study does justice to this research trend. Moreover, I fill the research gap in terms of health and well-being promotion of workforces in SMEs because enterprises are still pathogenesisoriented regarding health promotion of workforces (Kiesche, 2013). 
Salutogenesis as the basis of this study offers the opportunity to combine current health initiatives in companies with salutogenesis and its SoC criteria (comprehensibility, manageability and meaningfulness) and to investigate whether, or to what extent, current health promotion initiatives have the potential to promote the SoC, and therefore the well-being, of employees.

For the transfer of salutogenesis to SMEs in EWL/Germany, the study uses a practice-orientated research study by Bruch et al. (2013) called 'Healthy Leadership'. Bruch's study provides quantitative data for the topic of health and well-being promotion of workforces in the form of nine concrete initiatives for SMEs which are combined with the SoC factors by my study. Hence, my study aims to investigate whether, or to what extent, salutogenesis is applicable in SMEs in EWL/Germany and whether, or to what extent, current health promotion initiatives can promote the $\mathrm{SoC}$ of employees, and thus promote the well-being and health of employees.

The following sub chapter of the introduction provides a rationale regarding how and why health and well-being promotion is important in particular for SMEs in EWL in Germany. Antonovsky's theory of salutogenesis and the study by Bruch et al. (2013) about health promotion in companies are taken as the starting point for my research project into SMEs health and well-being promotion. Moreover, my background as a researcher is explained and the specific situation of SMEs in the region of East-Westphalia Lippe (EWL) is defined. This then provides the rationale for the research design.

\subsection{Embedding of thesis topic}

The challenges of the economic as well as the demographic development require companies even more than before to maintain and promote their employees as the most important resource of corporate success (Badura, 2010). In order to keep employees healthy and satisfied, promoting health in the workplace is a very 
important issue (World Health Organization (WHO), 2005). The Bertelsmann Foundation also emphasises the importance of innovative health promotion initiatives in companies in the future: "The promotion of health and the preservation of working and productive capacity will become an important task for the resource-poor location Germany in the future" (2011, p. 2 ,translated by myself).

Currently, German companies face many major challenges besides demographic development with their human resources strategies for example aging workforce, rising per-employee healthcare costs, and competition for a talented workforce (Bley et al., 2018; May - Strobl et al., 2015).

Human resources strategies of enterprises settled in the management belong to the field of 'Betriebliches Gesundheitsmanagement' (BGM) (Corporate Health Management) in Germany. The expert commission of the Bertelsmann Foundation (2011) in Germany claimed that in a modern approach of BGM or Corporate Health Management (CHM) sustainable processes and structures are developed with the help of management instruments to promote health in the workplace. Since the focus of my study is on top management and its awareness and valuations towards health and well-being promotion, I attribute the initiatives of my study to the field of BGM. To do justice to the English context of my study I use the international term of BGM 'Corporate Health Management' (CHM).

The idea of 'Corporate Health Management' (CHM) goes back to the Ottawa Charter of 1986 which aims to empower the population to deal with their own health as well as to shape the environment and health services. On the other hand, it is rooted in occupational safety and health, which has a long tradition and has been strengthened by European legislative initiatives in recent years and has a high level of professionalisation and institutionalisation (Badura et al., 1999). In addition to comprehensive health and safety regulations prescribed since 1996, CHM nowadays should also take into account workplace health promotion (WHP), the improvement of management culture, initiatives to reconcile private life and 
work, as well as tasks of age-appropriate work design into account. Thus, my study defines WHP as an important part of CHM.

CHM includes the systematic and sustained effort to design structures and processes to promote health and to empower employees (Bertelsmann Stiftung und Hans-Böckler-Stiftung, 2004). The aim is to create healthy conditions for workplaces and to encourage employees to behave in a healthy way.

Current studies show that investigations in WHP in Germany even show the monetary return on the investment of health promotion initiatives (Voermans, 2016). With effective WHP, employees' absences can be reduced by an average of $26 \%$. The cost-benefit ratio (return on investment, ROI) is extremely positive: with each Euro invested, 2.70 Euros can be saved through reduced absenteeism (Rau, 2015). Since 2009 occupational health promotion benefits amounting to $€ 500$ per employee and per year are exempt from tax in Germany. Businesses can implement initiatives such as exercise programmes, nutritional services, addiction prevention courses or stress management without tax assessment, provided they meet certain requirements (The National Association of Statutory Health Insurance Funds, 2017). In addition, companies with a strategical CHM are also rated more attractive by employees.

Summarised, CHM including WHP has many advantages for companies and can help to improve their employees' working conditions and could reduce disease rates (DAK, 2018). Further, companies are also competing for specialists and executives and want to increase their attractiveness by offering health promotion etc. Consequently, the promotion of health and well-being of the workforce in SMEs could be a support in the search for specialists and executives and an antidote to counter digitization, burn out etc. All these arguments have been confirmed by many studies and $80 \%$ of German companies recognise the necessity of CHM (Brinkmann, 2015). But why do only a third of them apply CHM? This is also confirmed by the Chartered Institute of Personnel and Development (CIPD) in the UK: "The concern is that the growing recognition of the importance 
of workplace health and well-being has not been matched by consistent and comprehensive action on the part of many employers" (2016, p.16). In addition, for $61.6 \%$ of SMEs in Germany CHM has a high priority but only $42.9 \%$ also pursue clearly defined goals (Lüerßen et al., 2015). Many SMEs do not have a clear strategy and a structured approach regarding health and well-being promotion as my professional consulting experiences shows.

Also, recent contributions from the government in Germany regarding occupational health and safety legislation which should take the psychological strain on employees into account, has not yet led to any rethinking in companies. The consideration of psychological stress in the risk assessment is a labour law obligation in Germany since 2013 but many companies are still at the beginning when it comes to taking account of the psychological stress in the risk assessment; even large companies with well-developed occupational safety and health structures and resources are often still in the development and testing stage (The Federal Institute for Occupational Safety and Health (BAuA), 2018).

It is noteworthy that it is difficult to win SMEs for the topic of health and wellbeing promotion of workforces. But it also raises the legitimate question which motivation (beyond the legal obligation or government funding) plays an important role for SMEs to promote the health and well-being of their workforces. Consequently, this thesis should be a contribution to the integration of workplace health promotion (WHP) as an important part of CHM in SMEs in EWL/Germany to promote the health and well-being of employees.

The question arise which WHP initiatives can specifically help SMEs in EWL/Germany or do SMEs need special WHP initiatives that meet the needs of SMEs? To answer this question one has to look first at the development of $\mathrm{CHM}$ initiatives since the 1970 s and 1980s when a strong focus was placed on occupational safety (Badura et al., 2003). The increasingly complex world of work (vast technologies, global trends etc.) nowadays brings with it new risks. Where only physical disorders concerned individuals in the 90s they "are now at risk from 
other organisational and wider environmental pressure" (Chartered Institute of Personnel and Development (CIPD), 2016 p. 11). More and more people seem to be affected by the consequences of permanent stress in Germany (Office for National Statistics 2015). A survey of the German insurance company 'Techniker Krankenkasse' (Health insurance for technical workforce) stated that employees feel stressed because of the work overload (Voermans, 2016). For decades the number of mental health related sick leave days has increased significantly: in the last 11 years by more than 97 \% (Initiative New Quality of Work (INQA), 2019). The German Labour Office (Arbeitsamt) released figures in 2012 stating that 60 million sickness related absence days are registered due to mental disorders (2014). Absenteeism and the following costs caused by stress and mental health conditions were a cause of concern also in the UK (Chartered Institute of Personnel and Development (CIPD), 2016 ; PricewaterhouseCoopers, 2014). Absenteeism statistics say a lot about the health status of a company but little about the true state of employee health because not every absent employee is sick and not everyone present is healthy (Badura, 2014). Therefore, absenteeism statistics do not show the reality of employees' health status and should be considered in a differentiated way.

In addition to absenteeism, there are other implications when it comes to ill mental health of employees. In Australia it has been recognised that mental illness is one of the main health related reasons for reduced work performance (Harvey et al., 2014). The increased turnover rates in the UK are also a reason for ill mental health of employees (Brand et al., 2017). In addition, mental illness is the leading cause of long term work incapacity in most developed countries today (Cattrell et al., 2011; Murray et al., 2012; Shiels et al., 2004). Of course, there might also be further influences contributing to staff sickness which are yet to be understood scientifically.

Nevertheless, the growing absenteeism, the reduced work performance and the increased turnover rates etc. in businesses should get more attention, and lead to 
a rethinking of health promotion of employees. It seems that it is less about offering back exercises in the company today but rather getting the support and understanding from the top manager (Matyssek, 2012). My study meets the changing needs of $\mathrm{CHM}$ by focusing on health and well-being of employees. According to the WHO (2013), well-being is a state of mental health in which an individual can realise his or her own potential, cope with the normal stresses of life, work productively and make a contribution to the community. The term 'wellbeing' means in German language 'Wohlbefinden' and refers to mental health (Psychische Gesundheit). 'Well-being' and 'mental health' are very similar terms that draws on usage in the German and English language literatures and that clarifies that the approach taken in this research follows a German-centric position. Moreover, I decided on the term 'well-being' because it is a term SMEs are more familiar with.

\subsection{My professional background}

I began my professional education in pedagogy at the University of Bielefeld and the Heinrich Heine University in Düsseldorf because I wanted to help and support humans with my further work. During my study I was on work placements in the local community for example women's shelters and youth welfare services. I found it sometimes emotionally challenging to work with some of the service users and their problems. I have learned to accept that humans cannot easily change or leave their often problematic environment (for example the family or partner), and my support often encountered limitations. Therefore, I decided to end my social work in the community after my graduation. Following an international trainee programme in economics, I decided to support and coach employers and employees in their working context as a consultant at the Centre for Competence of the University of Applied Sciences (Fachhochschule des Mittelstands (FHM)). Therefore, as a consultant and coach, I have found the matching area of consulting and coaching to optimise both, the company 
structures and, on the other hand, to support those peoples who work in the company structures. Professional support for me as a person makes sense if it is comprehensive. My study does not only consider the top manager side, but also the employee side, and takes also into account the business size of SMEs and their special structures as well as their employees' needs.

Since 2018 I have also been working also as a trainer for the University of Applied Sciences (FHM) and qualify business consultants as adviser of the support organisation 'Proactive SMEs' (called 'Offensive Mittelstand' in Germany). This support organisation is a national initiative in Germany. The partners have come together to promote a new quality of work in Germany for SMEs based on the guiding principles (www.offensive-mittelstand.de). As part of my working activities in this organisation, I am able to expand my knowledge of SMEs in Germany in addition to my work as a consultant and coach.

\subsection{The research region and the research sample}

My study takes place in a region in the east of North Rhine-Westphalia Germany called East-Westphalia Lippe which is characterised by many small and mediumsized enterprises (SMEs). It has been noticed as a region that produces success regarding health and well-being promotion in the economic fields (Gohs, 2005). 'The Association for the Promotion of Innovations in the Health Economy OWL e.V.' with their 'Center for innovation in the health industry East-Westphalia Lippe/Germany' is an initiative or network for health promotion. The aim of the network is to promote innovation in order to strengthen the economy and employment in the health sector and to promote the region of EWL/Germany as a location for the health industry. Hence, this region offers me a research arena for the study in selected SMEs to which I have access in a professional capacity.

Small and medium-sized businesses (called 'Mittelstand' in Germany) are often referred to as the heart of the Germany economy because they provide most of 
the economic output, train and employ the majority of employees, and contribute significantly to corporate tax revenue in Germany (Federal Association of German Industry e.V. (BDI), 2019). My study focusses on this type of enterprise in Germany and uses the international term 'SME' for this economic business size.

SMEs have a quantitative and qualitative character and meaning which is reflected in two different definitions. The quantitative definition is essentially based on statistical data (number of employees, annual sales etc.). The Institute for SME Research (IfM) (2019) defines companies with up to nine employees and/or less than $€ 1$ million annual turnover as small, and those with ten to 499 employees or with annual turnover of $€ 1$ million to $€ 50$ million as medium-sized companies. The totality of SMEs thus comprises all companies with less than 500 employees or $€ 50$ million annual turnover. There is a demand to continue to grasp SMEs because successful and fast-growing medium-sized companies will eventually cross the staff limit and be legally treated from this point on as large companies which does not do the company justice (Röhl, 2017).

However, following the qualitative definition of SMEs of the 'Institute of SME Research' (IFM), SMEs are usually family-owned business where the top manager exerts a significant personal influence, where the top manager bears the entrepreneurial risk and the company secures his personal income and livelihood. Accordingly, all companies belong to SM businesses (SMBs) in Germany which are family-run, no matter how small or big they are. As these characteristics are difficult to measure, quantitative characteristics are used so that SMEs can be distinguished from large companies as criteria of turnover, number of employees etc.

My research follows the quantitative definition of SMEs and involves companies with a small size, with a medium size and those which start to grow up to a big company (based on the number of employees and annual turnover) and thus have different top management structures. Therefore, the top managers of the chosen 
companies are made up of top managers who are owners, agents, masters or senior members of the executive board.

Each of the chosen companies is represented by at least one or two top managers of the management board. Hence, my study represents the economic region of EWL with its different sized SMEs and their different management board structures (Statistical yearbook North Rhine-Westphalia, 2014).

The SMEs and their top managers are selected based on companies to which I have access or connections in a professional capacity. Further, I chosse only SMEs where the top managers have leadership experience and those who have expressed that they are interested in health promotion of the workforce and are willing to discuss the theme with other SMEs. The type of company is here not of concern. It does not matter what type of business the company undertakes because health and safety regulations imposed by law are not considered in this study. What is of importance and relevance to the research study are top management awareness and attitudes towards health and well-being promotion of their workforce.

The economic existence of SMEs is often synonymous with the economic existence of the owner and his family. Although not all SMEs in Germany are family-run but there the intersection of family businesses and independent SMEs is very large (IFM, 2017).

In SMEs the direct influence of the top manager (if owner or not) in all areas of the company is noticeable. Due to the company size of SMEs it is possible for top managers to keep personal contact with the employees. An essential characteristic of SMEs is the strong influence of the owner or managing director (Voermans, 2016). This also applies to the health behaviour of the top managers. In SMEs, the health behaviour of the top management characterises the health culture in the company (Bruch et al., 2013). Furthermore, as owners and business leaders, they set the strategic direction of the company and shape the corporate culture. The direct influence of top managers in SMEs characterises the relationship between 
management and employees as well as between companies and their environment, and also influences the type of financing and choice of legal form.

SMEs typically have a flatter hierarchy because of their lower number of employees than large companies. But as soon as SMEs get bigger there are more decision makers (also at the top) besides the owner. "As the number of employees increases, the hierarchy levels increase: In companies with up to 20 employees, there is usually a management level, in companies with 21 to 99 employees, there are two levels, and in companies with 100 or more employees, there are three levels" (Sattes et al., 1998, p. 21 ,translated by myself). Therefore, it could also be assumed that the size of the number of employees in SMEs increases size of the management board. The patriarch who leads on his own does not match the reality in SMEs (Tödtmann, 2011). The management of a small business often consists solely of the owner and sometimes an experienced crafts man, a senior or a deputy belongs to the management (Initiative New Quality of Work (INQA), 2019). A management study indicates that only $33 \%$ of SME owners lead the company by themselves, $33 \%$ of SMEs were leaded by a duo, $17 \%$ by a trio and $16 \%$ of SMEs have four or more managers at the top (Tödtmann, 2011). My study accounts for these structures of SME management and involves two top managers of each SME (one top manager failed to attend).

There are different terminologies within German SMEs for managers who belong to the top management such as Chief Executive Officer (CEO), managing director or senior manager etc. regardless of whether they are owners of the companies or not. Within my study I use the term 'top manager' for all participating managers of the focus group because they are all decision makers in their company.

The participating SMEs of my study are family managed and two top managers of the focus group are also owners. The Institute for SME Research (IfM) classifies all companies as family businesses in which up to two natural persons or their family members hold at least $50 \%$ of the shares of a company and these natural persons belong to the management. 
Riess (2013) notices that family-owned businesses can think and act across generations because responsibility is anchored in their family tradition and values. The combination of risk and liability would lead to particularly responsible behaviour. Hence, an owner has internalised this principle because they want to pass on their heritage to their children and grandchildren. Owners of a family company invest their own money. Consequently, the long-term effects of their behaviour play a major role for them. Social involvement of family owned companies comes from family tradition and a grown corporate culture (Riess, 2013)

The sense of responsibility of family businesses is not reflected in the commitment of health promotion to employees and it cannot be confirmed that family-owned businesses are exemplary in their perceptions of economic, ecological and social responsibility (Riess, 2013). In addition, Gröben (2008) notices that ownermanaged companies spend significantly less on health initiatives than not ownermanagement companies. Further, it should also be noted that top managers of family-owned businesses often do not consider employee health and well-being as their responsibility or sole responsibility as my professional consultant experience shows.

Furthermore, it has to be taken into account that it is a challenge especially for owners of SMEs to take on additional tasks such as the implementation of CHM. Family relationships are closely linked to business relationships and roles are often not clearly defined. Especially for female owners often a problem arises from the multiple burden of family and work (Ammel et al., 2015).

Consequently, it cannot be assumed per se that owners are more open to health promotion than employed top managers. It is more useful to look at the resources and lower capacity of SMEs when it comes to promoting health of workforces in SMEs. Therefore, for my research it is not important if the top manager owns the company or comes from a family owned company. 


\subsection{Rationale for research design}

The appropriate method to receive the knowledge of a specific topic of health and well-being promotion and a specific group of participants of SMEs in EWL/Germany is focus-group discussions. In contrast to the methods of questionnaires or interviews, focus groups can be used to examine not only what people think but how they think and why they think this way (Kitzinger, 1995). This is especially important, because I want to know why health promotion initiatives can be easier, harder or impossible to implement in SMEs. Furthermore, group processes can help people to explore and clarify their views. In exchange with others they can simultaneously think about new topics of health promotion and reflect on them or critically discuss with others. Consequently, the method of focus-group discussions is more likely to suit the study research questions:

1st Research question: What salutogenic factors can be generated by top management to support the promotion of health and well-being of the workforce?

2nd Research question: How can SMEs develop top management awareness to keep their staff healthy?

3rd Research question: How can SMEs in East-Westphalia Lippe/Germany integrate salutogenic factors to promote the health and well-being of their workforces?

The question about who possesses the necessary knowledge to answer the research questions is imperative because to gain the required information it is mostly needed to ask various participants, which have different roles (employees or employer) and different experiences or perceptions about health promotion factors.

Consequently, I select participants from top management and employees from SMEs for the focus-group discussions. I anticipate getting additional information about health promotion factors from employees because they are likely to look at 
health promotion factors from a different perspective. They can supplement themes of their employers or discuss entirely new themes regarding WHP. However, in order to receive the required information for this research, the top managers are as important as their employees.

The sampling strategy and methods of data generation are further discussed and justified in more detail in chapter 2 'Methodology and methods'.

\subsection{Understanding of health and well-being in SMEs}

To support SMEs in EWL/Germany regarding health and well-being promotion of workforces my study takes the physical and mental side of health of employees into account. "[...] findings from the research literature suggest that developing a mentally healthy workplace is worth pursuing for its multiple benefits to individuals as well as organisations" (Harvey et. al, 2014, p. 12). Therewith, my study does justice to the current trend in health promotion focussing on the mental side of employees' health.

\subsubsection{Salutogenesis}

Salutogenesis has an influence on health promotion like no other model (Franzkowiak et al., 2011) and also as an interdisciplinary and integrative model salutogenesis is well suited to integrate it in different areas (Wydler et al., 2010). Therefore, the model of salutogenesis offers my study an ideal theoretical framework for the research field of health and well-being promotion in SMEs. Lindström et al. (2005) confirm that salutogenesis could create a solid theoretical framework for health promotion.

The SoC is the core component of salutogenesis, according to Antonovsky (1979, $1987,1996)$, and represents an important personal resource where it pays to take a closer look. "Salutogenesis was situated by Antonovsky as a question: what are the origins of health? His answer was the Sense of Coherence" (Mittelmark et al., 
2016, p. 10). Fundamental in the salutogenic framework is to consider health as a position on a health ease/dis-ease continuum and the movement in the direction towards health (Antonovsky, 1979). Therefore, the key elements of salutogenesis are the orientation towards problem solving and, secondly, the capacity to use the resources available (Lindström et al., 2005). Salutogenesis differs greatly from the pathogenesis that puts the search for individual pathogenic influences, the stressors, at the centre of their research. The coping resources play a special role here, ensuring that the person approaches the health pole as far as possible.

Antonovsky suggests that a strong SoC is the key determinant in the maintenance of health. He theorises that people with a strong SoC have the ability to (1) define life events as less stressful (comprehensibility), (2) mobilise resources to deal with stress-induced situations (manageability), and (3) possess the motivation to cope (meaningfulness) (Stickley et al., 2015; Wolff et al., 1999). Salutogenesis answers the question what kind of help humans need and raises the question of how humans despite the ubiquity of stressors (meaning adverse living conditions to which humans are permanently exposed) can remain healthy (Antonovsky, 1985, $1988,1997)$. In other words, it is about understanding health as a process where challenges and their stressors constantly appear and ideally meet appropriate resources.

In the context of appropriate resources of employees, the term 'resilience' appears again and again in titles of health promotion initiatives. The resilience concept and the model of salutogenesis have a lot in common and agree that they use resources or protective factors to emphasise positive-promoting conditions of health rather than rely exclusively on risk factors and pathogenic factors (Faltermaier, 2012). But salutogenesis is more health related than the resilience model, and it is more elaborate and theoretical (Lyssenko et al., 2011). In my view, salutogenesis offers the greater significance as a health science framework than the method-orientated resilience model. 
Moreover, salutogenesis fits well with my research field because WHP in SMEs should constantly adapt to new challenges and thus understand the integration of WHP as an ongoing process. As Lindström and Eriksson (2005, p. 440) perceive "salutogenesis has become an established concept in [...] health promotion." In addition, the WHO drew upon salutogenic theory in its benefits for the Health and Development Programme (European Union (EU), 2010; World Health Organization (WHO), 1986). The SoC model is successfully used in various professional fields for example medicine, nursing, sociology and psychology (Mittelmark et al., 2016). There are certainly several critical voices when it comes to the implementation of salutogenesis in practice. A key aspect is the changeability of SoC in adulthood. Antonovsky (1997) himself doubts the idea of influencing the SoC in adulthood. Nevertheless, Antonovsky sees the possibility of the SoC's temporary slight modification to avert harm and alleviate suffering (Wydler et al., 2010). Thus, it is about the strengthening of a weakened SoC of a human being and not about the general modification of the $\mathrm{SoC}$ of a human being. This study is based on the attitude that I do not fundamentally modify the SoC of employees in SMEs through the health promotion initiatives but do strengthen the SoC of workforces to stay healthy in the context of operational challenges in SMEs.

However, salutogenesis causes rethinking when it comes to health promotion of humans because it focusses on positive issues rather than on negative issues (as the pathogenesis does). As a researcher, I have also a personal reason to focus on the salutogenic perception. Focussing on positive issues fits well with my attitude as a person and as a coach or consultant in my business life. To promote health and well-being instead of developing a system for managing illness appears to be more successful than the other way around as the conventional system of treating patients has caused increasing numbers of absences from work in the last years (Voermans, 2016). Besides, Heyse et al. (2004) notice that reinforcing strengths merits more success than focusing on weaknesses, as has been evidenced by numerous behavioural research studies. 
With the theoretical framework of salutogenesis with focus on strength my study is associated with the science of positive psychology. The science of Positive Psychology is attributed to Abraham Maslow. He can rightly be described as the 'grandfather of Positive Psychology', as he has both coined the name Positive Psychology and postulated fundamental principles of positive human development (Blickhan, 2015). Positive Psychology deals in theory and research with the question of what that makes life worth. Further, it should help people to use their strengths and experience positive feelings. It contains topics such as experiencing the meaningfulness of one's own life, balanced contentment and situation-appropriate use of one's own talents (Harzer et al., 2017). No other area of psychology has made the transition from research to practice so quickly, and as Seligman (2017) claims that it is now time to make the knowledge available to those who can work with it.

Joseph et al. (2017, p. 83) compare Positive Psychology with the concept of salutogenesis and note "[...] it would seem that the relatively new field of Positive Psychology had much in common with the earlier approach of salutogenesis. Interestingly, however, the concept of salutogenesis has received relatively little attention within the Positive Psychology literature". However, my study does justice by integrating health and well-being initiatives in terms of salutogenesis and in terms of Positive Psychology.

A further reason to apply the SoC model in SMEs is the explanation about the origin and the preservation of health of humans. Knowing about the origin of health and also importance of well-being within can help SMEs to attach more importance to health and well-being promotion of employees (Pundt et al., 2016). In addition, salutogenesis identifies concrete variables (SoC factors) with which I can continue to work well to test them in SMEs. Hopkins et al. (2015) noticed that a change of perspective in health promotion in enterprises focussing on factors that support human health and well-being has not taken place yet. Although the health promoting effect of the SoC is empirically well documented (Wydler et al., 
2010), the role of the construct in the workplace is considered under-researched as Mette (2017) noticed.

The evidence that the promotion of health rather than the managing of illness is becoming a core consideration for successful enterprises grows stronger every day (Badura et al., 2017). In addition, the WHO agrees that the business sector becomes a key factor in promoting health in the future (2005). Thus, it is important for the largest and most important economy in Germany, the SMBs, to fundamentally re-examine the topic of health promotion of workforces. But there is a lack of using health and well-being promotion in SMEs (Lüerßen et al., 2015). My study fills that gap and aims to help SMEs with the knowledge of salutogenesis in their efforts to maintain and promote employees' health and well-being. Summarized, by using salutogenesis with its SoC model, my study could help SMEs to meet their future challenges by:

- knowing how health is created,

- knowing that well-being is an important part of health promotion,

- focussing on positive issues and resources instead of managing a system of illness.

\subsubsection{Research study about developing a healthy performance culture in} SMEs by Bruch et al. (2013)

In order to get an acceptance of the theoretical SoC model in SMEs in EWL/Germany it is necessary to present it in a practice-oriented, or applicationoriented, form. Top managers of SMEs are generally more open to practice-related topics than to theories (they often call studies impractical or abstract) as my coaching or consultant experience shows. Therefore, it is important to find a current study that provides quantitative data for the topic of health and well-being promotion of workforces in SMEs from which then recommendations for action for SMEs can be derived. 
On the one hand, the practical reference of study data is needed to implement it in SMEs. On the other hand, a concrete description of initiatives that are drawn from the study findings is needed to decide which of the SoC variables (meaningful, manageable or comprehensible) is promoted by the health promotion initiatives of the study. So, the description of health promotion initiatives within a study allows the link to salutogenesis in my study. Summarising, I need quantitative data from SMEs which are prepared in such a way that they can be directly tested for application in SMEs in EWL/Germany and bring findings that make it possible to connect to the SoC model of Antonovsky.

The study 'Healthy leadership: How do companies develop a healthy performance culture' by Bruch et al. (2013) fulfils the conditions and is therefore used as a basis for my research project. The study by Bruch et al. (2013) is developed explicitly for use in SMEs and is developed as part of the German Initiative 'TOP JOB'. TOP JOB has been measuring employer attractiveness of SMEs since 2002, helping them to further develop their qualities as employers in a targeted and measurable manner. The methodology is designed by the University of St. Gallen exclusively for TOP JOB. With the associated employer seal 'TOP JOB - Top Employer', TOP JOB supports the participants in employer branding, in building up a more attractive employer brand, and in recruiting. TOP JOB is a project of 'Zeag GmbH - the centre for employer attractiveness' at Lake Constance, Bavaria, which also organises the project" ETHICS IN BUSINESS", the value alliance of SMEs.

Since 2005 Prof. Dr. Heike Bruch has been the scientific head of TOP JOB and has also taken over the evaluation of the data of the study 'Healthy leadership' with her Institute for Leadership and Personnel Management at the University of St. Gallen.

Bruch et al. (2013) analyse the survey results of 15,544 employees, 264 managing directors and management board members as well as 96 personnel managers from 96 German companies. The average response rate is $72 \%$. The catchment area as the entire Federal Republic of Germany with the following geographical 
coverage: $26 \%$ of companies are from Baden-Württemberg, $24 \%$ from North Rhine-Westphalia, 18\% from Bavaria and 10\% from Lower Saxony.

The other federal states are represented in the single-digit percentage range. The companies are divided into three size categories: small companies (20-100 employees), medium-sized enterprises (101-500 employees) and large companies (501-5,000 employees). The average number of employees is small 57 employees, medium-sized companies 261 employees and large companies 1,297 employees. The sectors are as follows: $55 \%$ of the enterprises come from the service sector, $24 \%$ from the manufacturing industry, $12 \%$ from the trade and $9 \%$ from finance, insurance and real estate.

The 96 participating companies of the study by Bruch et al. (2013) participated in the employer-benchmarking TOP JOB. This could mean that companies who are interested in a quality seal of TOP JOB are perhaps more innovative in terms of health promotion than other companies who do not undergo this seal of approval. That could also explain the large response rate of the study by Bruch et al. (2013). Therefore, it must be considered for my research project whether or to what extent the recommendations of the study by Bruch et al. (2013) in SMEs are to be implemented in EWL/Germany.

Although the study surveys 15,808 people, only a few are decision-makers in companies. In other words, the results of the study do not reflect the top management and employee opinions in the same way. My research project wants to create this balance and integrate the top management more. It should also be noted that the study results are also based on surveys of large companies (up to 5,000 employees). According to the quantitative definition of SMEs in Germany, some participating companies could not be counted among the SME class. In addition, the study gives no information about the company size of the 96 participating companies. So, it could be that the participating SMEs in my study cannot, or only partially, identify with the health promotion initiatives of the study by Bruch et al. (2013) because they do not have any additional resources and/or staff for the health promotion of their employees. This means for my study that I 
pay attention to whether my chosen SMEs in EWL/Germany can identify with the initiatives of the study by Bruch et al. (2013).

The results of the study by Bruch et al. (2013) show the extent to which healthy leadership by management influences well-being and health in the company. For Bruch et al. (2013) healthy leadership is given when top managers feel responsible for the health of their employees, be mindful of them and be good role models with their own health (2013). According to the study, the behaviour of the top management and executives regarding their own health also has great importance for the mental health of their employees within the company.

The study published a guideline for enterprises on how to promote health and well-being of workforces (see appendix 9.2, 9.3). The guideline includes nine recommendations for health promotion of workforces in enterprises. No other representative study focusing on health and well-being promotion in SMEs in Germany provides such a concrete action plan that can be used directly in SMEs. The reason for this application orientation of the study by Bruch et al. (2013) is that this study has been explicitly created for implementation in SMEs. The required prerequisite for my research project is given with the concrete recommendations of the study how German companies manage to promote the health and well-being of employees,

The data of the study by Bruch et al. (2013) is gathered with a questionnaire consisting of a combination of multiple-choice and open questions. Statistical mean comparisons and median splits are used as analytical methods. Thus, the research method and the results of the study by Bruch et al. (2013) indicate what companies can do to promote employee well-being but do not say how they can do it or if it is possible to integrate it in SMEs. Furthermore, the quantitative method of the study leaves only little opportunity to explore which ideas the companies have to promote health and well-being of the employees. Hence, the aim of my research study is also to explore quantitative data through focus-group 
discussions to gain a deeper insight and understanding on how SMEs can integrate health and well-being promotion initiatives in EWL/Germany.

The concept of the study by Bruch et al. (2013) is also based on the analysis of leadership behaviour or leadership styles regarding health and well-being of employees but does not pay enough attention to the needs and values of top managers in SMEs. As mentioned before the top manager should receive a greater value in my research project than in the study by Bruch et al. (2013). Since top managers are the decision makers, it is of great importance to explore their assessment and awareness of this topic. Consequently, my study research questions explore how they rate or value initiatives and why do they rate them? With the help of my qualitative research, I contribute to the value of top managers of SMEs regarding health and well-being promotion. It is important to know what values and beliefs top managers have in SMEs in order to facilitate the implementation of health promotion initiatives. Top managers are increasingly leading companies in their old age and health promotion should therefore address the age of the top managers or decision makers. "Those who are still there are tenacious, preferred by family businesses [...], in positions where they can do a lot. Therefore, it is worth taking a look at the values and characteristics of this generation" (Bund, 2014, p. 7 ,translated by myself). The generation of older top managers in SMEs with their values could have an impact on the promotion of well-being and health of employees. In late wartime or post-war generations, the top managers relinquished their claims and were primarily concerned with the business. They do not know, or are not concerned, about well-being or mental health of their employees, and thus could be the reason why this topic has not been addressed by them before. However, my study explores the values top manager hold and the origins of their values and put them into the context of the promotion of health and well-being by discussing it in relation to current WHP research. 
In summary, the study by Bruch et al. (2013) offers many important findings and recommendations regarding health and well-being promotion in SMEs. But the exploration of the applications in SMEs is still missing. My study fills the knowledge gap in the field of SMEs in EWL/Germany. In addition, I do justice to the trend of health promotion in the sense of salutogenesis by linking to the recommendations of the study by Bruch to the components of the SoC model by Antonovsky. Consequently, with the results of the study by Bruch et al. (2013) and its link to the SoC model, my study can explore whether, or to what extent, salutogenesis is applicable to businesses, especially to SMEs, today.

\subsection{The study concept}

The study by Bruch et al. (2013) is used as a transformation of salutogenesis into SMEs practice in EWL/Germany. With this link salutogenesis could maintain its current status and could also get an acceptance in SMEs. The connection is used to investigate which of today's WHP investigations correspond to the criterions of the SoC model, and thus promote the SoC of employees or the well-being and health of employees.

Therefore, my study complies with the request of health sociology to provide salutogenesis in an innovative way for practice (Wydler et al., 2010). Because the SoC model is a complex model, I also fulfil the research gap for a better practicability or applicability of the SoC (Grabert, 2011; Wydler et al., 2010).

The study by Bruch et al. (2013) announces nine concrete initiatives to develop a healthy corporate culture in SMEs (see appendix $9.1 \&$ 9.2) which focus on human needs like self-esteem, trust, appreciation and self-responsibility. The following table shows the short version of the guideline within the study by Bruch et al. (2013) and gives an overview (the original see appendix 9.2). 
Table 1: Nine topics of the guideline (short version) within the study 'Healthy Leadership' (Bruch et al. (2013), translated by a translation agency).

1. Top management as pioneers of a healthy performance culture: A company's top management is central to a healthy performance culture. Managing directors are internal trendsetters, who can use their role to initiate, promote and consolidate a healthy performance culture.

2. Healthy self-management: Executives have a classic dual role, especially when it comes to health. However, the majority of managers do not see 'self-care' as a key issue. Instead of a "I take care of myself", managers are more likely to say "there is more asked of me than of my coworkers".

3. Holistic health management: In many companies, workplace health management is still in its infancy. The various measures, which are brought together in companies under the heading of 'occupational health management', are more effective at promoting health when they are interlinked and tailored specifically to the needs of the company and its employees. However, holistic health management still boils down to two major steps.

4. Freedom with a safety net: The delegation and acceptance of responsibilities are both crucial for management and employees to meet increased requirements. The transfer of responsibility means management letting go of a responsibility and the employee taking it over, so the management needs to be able to trust them and have confidence in them.

5. Meaningful work: It is important that employees know the importance of their contribution to the achievement of company goals. Particularly in highly specialized companies, employees can find little to no connection between their tasks and the end product. This will raise major questions about the meaningfulness of one's own work.

6. Value: According to the WHO (World Health Organization), "health is a state of complete physical, mental and social well-being and not just the absence of disease or infirmity". Recognition and appreciation of one's own person are important for spiritual and social well-being and this goes beyond praise for very good achievements

7. Challenging and supporting: The roots of occupational health management lie in occupational health and safety and occupational safety. The realisation that working conditions are important to the health of employees and companies have a duty to work in the field of occupational safety has been an important milestone in the development of occupational health management.

8. 'Health consumption': Management, HR and executives have a duty to provide care for the employees, in particular for their well-being and health. However, every employee is responsible for his or her own health and must not be relieved of this responsibility due to misunderstood understanding of consumption in the sense of "I'm waiting to see what my management offers me".

9. Psychological 'immune system': Executives should shape the work environment of their 
employees so that requirements are in proportion to resources. Employees can, however, do a lot to improve their personal resilience and immunity to stress triggers. Exercises which build and promote resilience, which is to say resistance, are particularly helpful here.

The human needs that emerge in the nine initiatives within the study by Bruch et al. (2013) are also important prerequisites for strengthening a human's Sense of Coherence $(\mathrm{SoC})$. Thereby, the nine initiatives correspond well to the SoC elements, as health and well-being promotion needs to incorporate the importance of 'comprehensible', 'manageable', and 'meaningful' (see figure 1).

Figure 1: Health, stress and coping (Antonovsky, 1979).

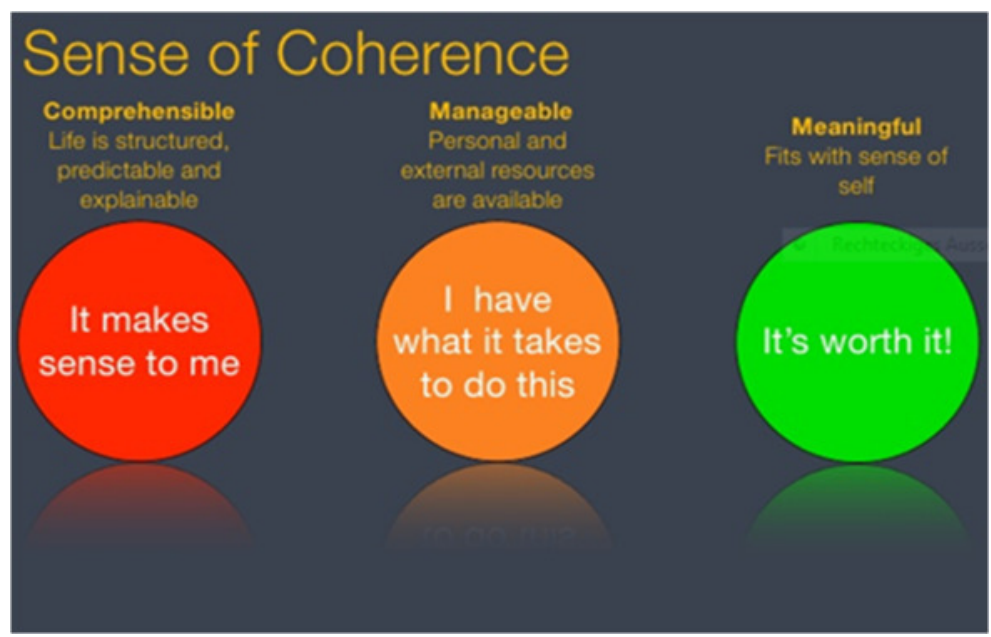

"SoC reflects a person's view of life and capacity to respond to stressful situations. It is a global orientation to view the life as structured, manageable, and meaningful or coherent" (Lindström et al., 2005, p. 441). Employees in SMEs therefore need to be able to understand the work contexts in their work, to feel the urge to do the work and to recognise a meaning and purpose in their work. The nine initiatives of the study by Bruch et al. (2013) seem to take into account the components of the SoC.

In the next step, the nine initiatives are sorted to the SoC element as defined by Antonovsky. Because of that, I check whether the nine initiatives primarily 
strengthen the feeling of comprehensibility, manageability or the feeling of meaningfulness of workforces. In other words, I examine which of the following points primarily are served by the nine initiatives by Bruch et al. (2013):

1. The ability to understand the connections of life (the feeling of comprehensibility), or

2. the conviction to be able to shape one's own life (the feeling of manageability or controllability) or

3. the belief in the meaning of life (the sense of meaningfulness).

For example, the measure 'top management as pioneers of a healthy performance culture' could promote primarily the comprehensibility of employees. If the top manager is a pioneer regarding health promotion, the employee could better understand why health promotion is also important to him.

The following figure shows the matching of the topics referring to the study by Bruch et al. (2013) to the SoC factors of Antonovsky's SoC model. Some topics can be assigned to several SoC criteria. 
Figure 2: Allocation of Antonovsky's SoC elements with the health promotion initiatives of the study by Bruch et al. (2013).
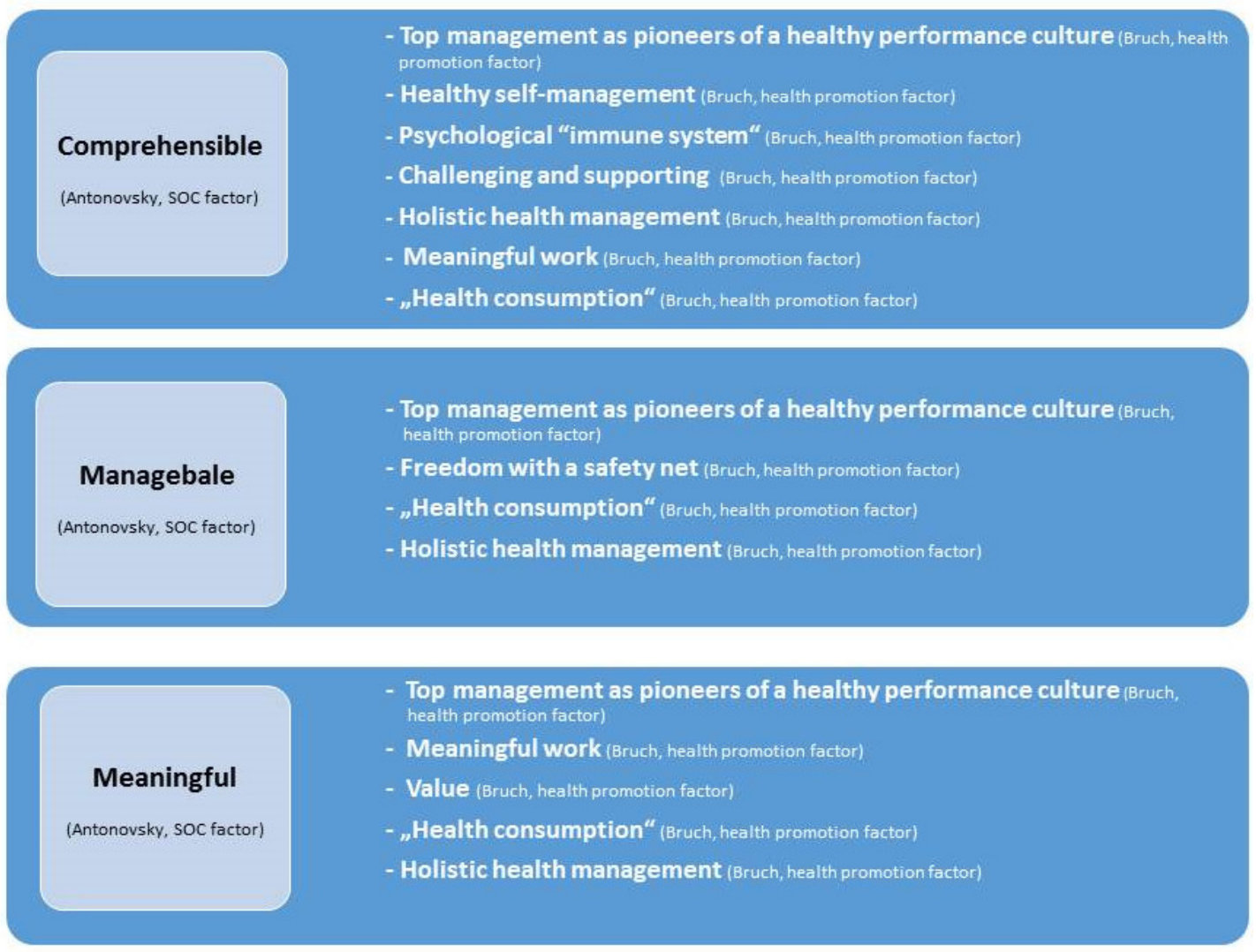

The combination of the SoC model by Antonovsky and the study by Bruch et al. (2013) makes it possible to meet the current trend in salutogenic health promotion of employees and makes up the originality of my study. Therewith, my study pursues a turnaround of health and well-being promotion away from the cause of disease research to the cause of what makes employees healthy. The testing of application into practice of salutogenesis in this form in SMEs in Germany is completely new and no study has yet been conducted in this form.

\subsection{Research questions and objectives}

An important and necessary base of business research is the identification of welldefined study research questions. The research questions guide the research project and assist in the construction of a logical argument. Further, research 
questions and objectives must be accompanied by the theoretical position of the researcher, his or her philosophies (see chapter 2.3), and the methodologies and methods employed (see chapter 2) (Bryman et al., 2011; Flick, 2012; Jankowicz, 2005; Polonsky et al., 2010; Saunders et al., 2011).

The theoretical framework of my study is based on the SoC model by Antonovsky (1979, 1996). In this way, well-being has a special significance within the framework of health promotion in my study because it has been proven that there are correlations between the SoC and various indicators of well-being (Antonovsky, 1997; Singer et al., 2007). Consequently, the research questions of my study relate to salutogenesis and focus on strengthening the SoC of workforces in SMEs in EWL/Germany. Therefore, it is important to explore first what salutogenic initiatives can be used by top management of SMEs in EWL/Germany to promote the health and well-being of their workforce. Further, my study wants to explore how top managers evaluate health and well-being promoting factors, and to understand how useful health and well-being promoting factors are for the top managers.

Figure 3: Structure of the three research questions (own figure).
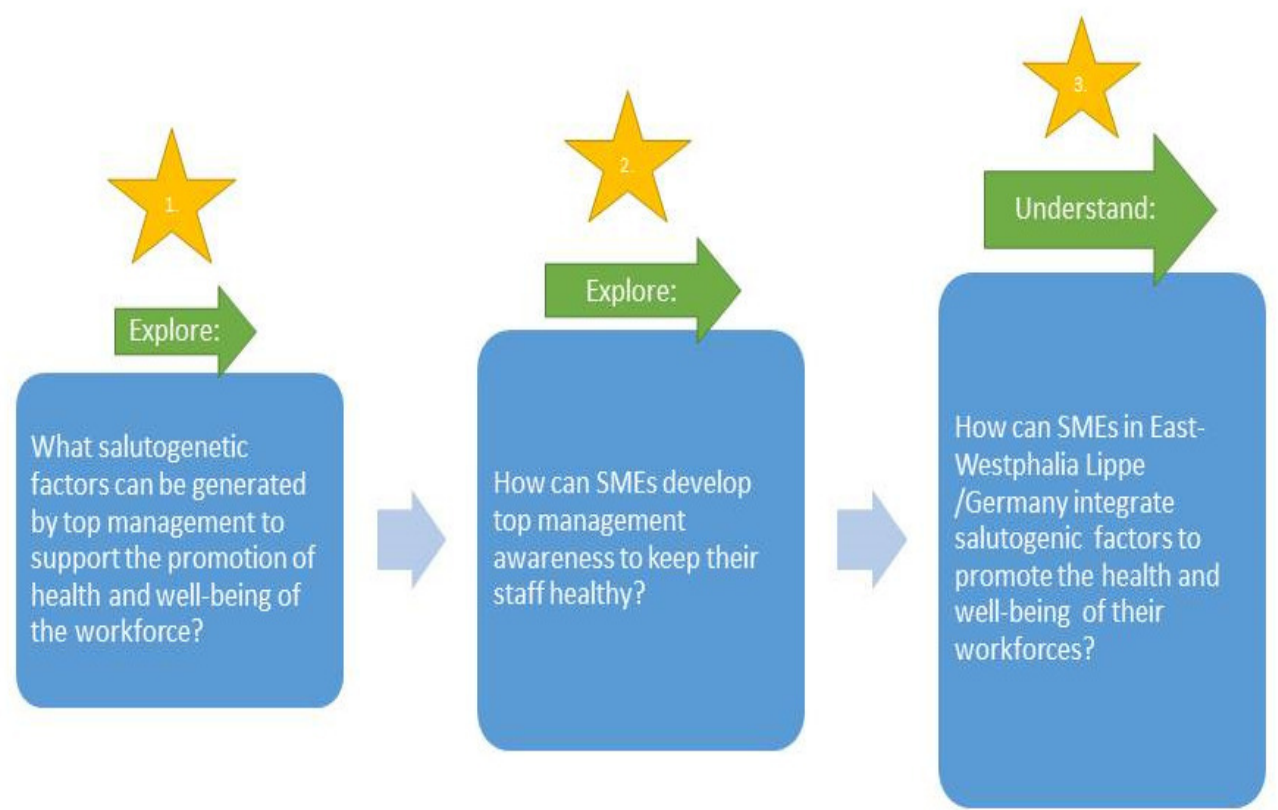
This leads to the following research question of my study:

\section{Research question 1:}

What salutogenic factors can be generated by top management to support the promotion of health and well-being of the workforce?

To achieve the aim of understanding the coherence between top management awareness and the support of health and well-being promotion of the workforce, two further research questions have to be answered.

\section{Research question 2:}

How can SMEs develop top management awareness to keep their staff healthy?

The following research question encapsulates the primary aim of this project.

\section{Research question 3:}

How can SMEs in East-Westphalia Lippe/Germany integrate salutogenic factors to promote the health and well-being of their workforces?

By showing the importance of top management awareness regarding WHP, it is possible to add something new to the already existing knowledge base. However, I would like to consider three issues: what salutogenic initiatives can SMEs apply, how can SMEs make top managers aware of the topic, and how SMEs can integrate salutogenic initiatives. So, it is important to think about how I get helpful information to answer my research questions.

I present the first research question with the help of the nine recommendations from the study by Bruch et al. (2013) that I have previously assigned to the SoC elements (see figure 2). Therefore, I present the current salutogenic initiatives to SMEs in EWL/Germany and let them evaluate their value and usability. Furthermore, I try to answer the second and third questions with the help of open questions which allows discussion of the participants' own ideas. There is plenty of room for participants to share their opinions and ideas on how to make top 
managers aware of health promotion in SMEs or how to integrate health promotion factors into SMEs.

The following table summarises how the study research question and research objectives are connected.

Table 2: Connection of research questions and research objectives.

\begin{tabular}{|c|c|c|}
\hline Research Questions & Research Objectives & Research strategy \\
\hline $\begin{array}{l}\text { What salutogenic factors can be } \\
\text { generated by top management } \\
\text { to support the promotion of } \\
\text { health and well-being of the } \\
\text { workforce? }\end{array}$ & $\begin{array}{l}\text { Nine prompts are presented } \\
\text { with regard to value and } \\
\text { applicability of health } \\
\text { promoting factors in form of a } \\
\text { scenario }\end{array}$ & $\begin{array}{ll}\checkmark & \text { Qualitative research design } \\
\checkmark & 2 \text { Focus group discussions (FGD). 1. FGD: top managers of } \\
\text { the three SMEs . 2. FGD: employees of the three SMEs. } \\
\checkmark & \text { Three to four hours in total will be collected } \\
\checkmark & \text { Recorded by an audio player } \\
\checkmark & \text { Data are dissected by the Thematic Analysis and are coded } \\
\checkmark & \text { by software NVIVO } \\
\checkmark & \text { Induction }\end{array}$ \\
\hline $\begin{array}{l}\text { How can SMEs develop top } \\
\text { management awareness to keep } \\
\text { their staff healthy? }\end{array}$ & $\begin{array}{l}\text { Open questions are asked } \\
\text { about developing top } \\
\text { management awareness } \\
\text { regarding workforce health } \\
\text { and well-being }\end{array}$ & $\checkmark \quad$ FGD (see above) \\
\hline $\begin{array}{l}\text { How can SMEs in East- } \\
\text { Westphalia Lippe/Germany } \\
\text { integrate salutogenic factors to } \\
\text { promote the health and well- } \\
\text { being of their workforces? }\end{array}$ & $\begin{array}{l}\text { Open questions are asked } \\
\text { about integrating health and } \\
\text { well-being promotion factors }\end{array}$ & $\begin{array}{ll}\checkmark & \text { FGD (see above) } \\
\checkmark & \text { Transferability based on the reliability of the data collected } \\
\text { are discussed in order to formulate recommendations }\end{array}$ \\
\hline
\end{tabular}

The objects of my study help me to explore which salutogenic initiatives SMEs can use to keep their employees healthy and how they can be integrated. The study objectives insist on surveying the nine initiatives of the study by Bruch et al. (2013) in the first step, and the open questions about how SMEs create the integration of health and well-being promoting initiatives and what they need for it. Moreover, the study objectives enable me to generate qualitative and detailed data to help SMEs in EWL/Germany in keeping their employees healthy. 


\subsection{Rationale of this thesis}

This study aims to help SMEs in EWL/Germany to help keep their employees healthy because they are the most important resource for businesses in the future (Bertelsmann Stiftung, 2011). Although SMEs provide most of the economic output and train and employ the majority of employees in Germany, they often do not have the resources for $\mathrm{CHM}$ as big companies do. Hence, the study confirms the conditions of this company size and prepare the study findings in such a way that they lead to WHP initiatives that do not require many company resources and are easy to implement.

As there is already a lot of quantitative data on WHP initiatives in SMEs, I have decided to generate qualitative data on salutogenic factors to supplement understanding derived from quantitative data evident in other work. For this, I choose a new way to support SMEs in promoting the health and well-being of workforces while using salutogenesis as a theoretical model in order to pursue a turnaround in health promotion away from the cause of disease research (pathogenesis) to the cause of what makes employees healthy (salutogenesis). Salutogenesis explains how health develops and explains what humans need to feel healthy and comfortable, and therefore salutogenesis could help top managers in SMEs to look at employee needs differently. They could understand that it pays to invest in humans.

In my view, it is important to support SMEs to focus on what could strengthen and support workforces (salutogenic way of thinking). It is just about focussing on positive issues and resources instead of managing a system of illness.

Moreover, salutogenesis is applicable interdisciplinarily and is successfully used in various professional fields for example medicine, nursing, sociology and psychology. But there is a research gap in implementing salutogenesis in the economy and above all in SMEs. 
To test salutogenesis for applicability or usability in SMEs, it takes a bridge, or a kind of transformation, to be accepted in SMEs. For this I use the guideline within a current research study called 'Healthy Leadership' by Bruch et al. (2013) which focuses on the promotion of health and well-being of employees in SMEs. The guideline of the study by Bruch et al. (2013) (which includes nine concrete WHP initiatives) can therefore be attributed to the SoC components. With the help of the study by Bruch et al. (2013) I explore which salutogenic initiatives SMEs can integrate and how they can be integrated to promote employee health and wellbeing. Finally, my research project aims to find out whether it is advisable for SMEs in the future to focus their efforts on strengthening the SoC of their employees.

\subsection{Summary of introduction chapter}

This introduction shows the urgent need for a new thinking about the health promotion of workforces in SMEs. The idea is to use the salutogenesis by Antonovsky as an interdisciplinarily and successfully used model (in various professional fields for example nursing). There is a research gap in implementing salutogenesis in the economy and above all in SMEs. To date, no data exits how to apply health and well-being promotion in terms of salutogenesis in SMEs. Therefore, it is necessary to explore an understanding of health and well-being promotion regarding salutogenesis in SMEs.

For the transfer of salutogenesis to SMEs in EWL/Germany the study uses a research study by Bruch et al. (2013) which provides quantitative data for the topic of health and well-being promotion of workforces in the form of nine concrete initiatives. These initiatives are combined with the SoC factors (Antonovsky, 1987) in my study. Hence, my study aims to investigate whether, or to what extent, salutogenesis is applicable in SMEs in EWL/Germany and whether, or to what extent, current health promotion initiatives can promote the SoC of employees, and thus promote the well-being and the health of employees. 
Summarising, the first chapter provides a rationale how and why health and wellbeing promotion is important in particular for SMEs in EWL in Germany. I take Antonovsky's theory of salutogenesis and the study by Bruch et al. (2013) about health promotion in companies as the starting point for this research project into SMEs health and well-being promotion. Moreover, I explain the background of the researcher and I explain the specific situation of SMEs in the region of EWL is defined. This then provides the rationale for the research design.

The introduction section provides a sound basis for the second chapter which presents an explanation of the study's methodology and the study research method. The following chapter explores the literature and research extant in this field and examines the literature available in this area and attempts to find 'Which salutogenic factors support the promotion of health and well-being of the workforce in SMEs?' The literature review and the following empirical research form the methodological base of my study. The last part of my study is the comparison and discussion of current research findings of the literature review with my study findings in order to derive recommendations for SMEs regarding health and well-being promotion of workforces.

There is a lack of using health and well-being promotion in SMEs (Lüerßen et al., 2015) which my study wants to fill. In addition, my study aims to help SMEs with the knowledge of salutogenesis in their efforts to maintain and promote employees' health and well-being to meet their future challenges. This raises the question 'Which salutogenic factors support the promotion of health and wellbeing of the workforce in SMEs?' which I want to answer with my study. The following chapter 'Methodology and methods' provides the research method that helps me to answer my study research question.

For the sake of easier readability, my thesis uses the familiar male form of personal nouns and pronouns, but this does not imply discrimination against the female gender but should be understood as gender-neutral in the sense of 
linguistic simplification. In addition, for the sake of easier readability I use the shorter term WHP (workplace health promotion) instead of promotion of health and well-being of employees. 


\section{Methodology and methods}

I choose a new way to support SMEs in promoting the health and well-being of workforces by using salutogenesis as a theoretical model in order to pursue a turnaround in workplace health promotion (WHP) away from the cause of disease research (pathogenesis) to the cause of what is, or makes, employees healthy (salutogenesis). That leads to the following review question which I want to answer with the literature review of my thesis: 'Which salutogenic factors support the promotion of health and well-being of the workforce in SMEs?' The review question has a connection to the study research questions, (especially to the first research question (see figure 3 )), which in turn are based on the rationale of my study.

This chapter begins with a description of the study aim to set the inclusion and exclusion criteria of my research project in a timely manner. Further, I will explain the research ethics which the study considers will be explained. Afterwards, the reflection of research philosophy on which this study is based, follows. Research philosophy authors such as Grix (2002, p. 180) suggest that it is best to proceed by iterative development in logical order starting from

$\checkmark$ Ontology (What is out there to know) via

$\checkmark$ Epistemology (What and how can we know about it?) to

$\checkmark$ Methodology (How can we go about acquiring the knowledge?) for

$\checkmark$ Collecting and analysing data and finally sources (Which data can we collect?).

At the end of this chapter I will display the research design with its methods and the analysis plan will be displayed. Finally, I will justify the systematic way of the literature review. 


\subsection{Study aim with its inclusion and exclusion criteria}

The model of salutogenesis offers my study an ideal theoretical framework for the research field of health and well-being promotion in SMEs and can be transferred by connecting the model with a current WHP study by Bruch et al. (2013) into small and medium-sized businesses in EWL/Germany. Accordingly, my study uses existing quantitative data from the study by Bruch et al. (2013) to test them for applicability in SMEs in EWL/Germany. Since the data of the Bruch' study results in concrete initiatives for SMEs, these can be assigned to the components of the SoC. With this it is possible to find out if, and to what extent, today's WHP initiatives strengthen the SoC among employees or if it helps SMEs to create or shape their WHP initiatives in the sense of salutogenesis. In other words, I am checking salutogenic WHP initiatives for applicability in SMEs in EWL/Germany to support SMEs to maintain or promote the health and well-being of their employees with my study findings.

The physical and mental aspects of health often coincide and play together (Bruecker et al., 2012), yet the study places a special focus on the well-being of employees because of its connection to salutogenesis. As stated in the first chapter, it takes a rethinking of $\mathrm{CHM}$ in companies from purely physical such as fitness or back exercises, to the promotion of mental health or well-being, as evidenced by the growing absenteeism caused by mental disorders of employees in Germany in recent years.

My study does not explore only leadership behaviour and its impact on the health and well-being of employees, since there are already enough studies available. Of course, the leadership styles of top managers of SMEs are part of my research but only keeping employees' health only in line with leadership styles would be wrong (Bruch et al., 2013). It is about more than just establishing the right leadership style in SMEs. To promote employees' health and well-being, SMEs should think holistically. My study explores more aspects than just leadership styles: e.g. the 
health behaviour of top managers, corporate culture in terms of communication, participation of employees, and appreciation of employees. Furthermore, I explore the personal responsibility of employees regarding health and well-being.

It should be noted that the success of a CHM system depends on the support of the top management in SMEs. If the need and understanding of top management is lacking, the success of healthy employees seems to be missing (Zimber et al., 2011). Therefore, my study involves decision makers in SMEs as they are the key when it comes to the health and well-being of employees.

The theory of salutogenesis has an influence on the concepts and on the public health scene, especially in the USA, Scandinavia and Australia (Blättner, 2015). It also has an impact on health promotion in the work context in Europe (Mittelmark et al., 2016), and is used mainly in the social, psychology and medical areas in Europe (Homfeldt et al., 1997). However, the idea of the salutogenic model has its beginning in the USA. Aaron Antonovsky published the main and most important books in the USA. Because of these points I limit the location of the literature in Europe, USA and Australia. Furthermore, I use studies written in English and German language because I am speaking these two languages.

The German Occupational Safety and Health law which came into force in 1996 sets the European framework directive 'on the implementation of initiatives to improve safety and health protection of employees' from 1989 into national law (Bruder et al., 2010). In the area of occupational health and safety legislation, it functions as a kind of 'Basic Law'. Esslinger et al. (2010) notice the legally prescribed occupational health and safety is an important part of occupational health management but a holistic $\mathrm{CHM}$ involves more and must be created by the company through its own initiative and commitment as a supplement to the statutory minimum requirements" (translated by the myself). Nevertheless, the German Occupational Safety and Health law of 1996 plays an important 
foundation for the implementation of health promotion in companies in Germany. With the Luxembourg Declaration, a Declaration on Workplace Health Promotion (WHP) was written in 1997 by the European Union, thus laying down a uniform idea of what health care criteria means in the workplace. The time frame of my literature research is based on a German legal adjustment and secondly on a declaration that is aimed at improving the quality of WHP in Europe. Both initiatives can lead to more attention in the research and practice of $\mathrm{CHM}$ from 1996 onwards, which explains the timeframe (1996-2019) of my study.

My research focuses on employees of SMEs, and I only include studies reporting on $\mathrm{CHM}$ or WHP are included. In addition, the model of salutogenesis offers my study an ideal theoretical framework for the research field of health and wellbeing promotion in SMEs. The physical and mental aspects of health often coincide and play together (Bruecker et al., 2012), yet the study places a special focus on the well-being of employees because of its connection to salutogenesis. As stated in the first chapter, it takes a rethinking of $\mathrm{CHM}$ in companies from purely physical WHP such as fitness or back exercises to the promotion of mental health or well-being, as evidenced by the growing absenteeism caused by mental disorders of employees in Germany in recent years. Further, the study takes place in small- and medium sized businesses (SMBs) in EWL and focuses on this special size of businesses.

In order to create a theoretical framework of WHP it is necessary to include studies which report on processes and results of employee health and well-being. I exclude studies which only focus on pathogenic factors. In other words, the focus is on the causes of health and well-being of employees and not on eliminating symptoms. The study thus has a positive and resource-oriented focus in the field of CHM in SMEs in EWL/Germany. Therefore, I search for studies with enablers of successful WHP via thematic analysis.

Based on Clarke and Oxman's study (as cited in Tranfield et al., 2003) only primary studies can contribute to synthesising evidence from various studies in the SME 
field. My study includes both qualitative and quantitative studies to get extensive knowledge about implementing health and well-being initiatives in SMEs in EWL/Germany. I do not include studies which do not report on methods applied (unclear methodology) for data collection and analysis.

The following table summarises the inclusion and exclusion criteria of my study and also forms the basis for the systematic literature review (see chapter 3 ).

Table 3: Inclusion and exclusion criteria of the study.

\begin{tabular}{|c|c|c|}
\hline Parameters & Inclusion criteria & Exclusion criteria \\
\hline Locations & Europe, USA and Australia & Non-Europe, non-USA, non-Australia \\
\hline Language & $\begin{array}{l}\text { Literature written in English and } \\
\text { German }\end{array}$ & $\begin{array}{l}\text { Literature NOT written in English and } \\
\text { German }\end{array}$ \\
\hline Time frame & Literature published after 1996 & Literature published before 1996 \\
\hline Subjects & $\begin{array}{l}\text { Workplace health promotion (WHP) } \\
\text { focusing also on mental health }\end{array}$ & $\begin{array}{l}\text { Workplace health promotion (WHP) } \\
\text { focusing only on physical side }\end{array}$ \\
\hline Relevance & Salutogenesis & Pathogenic or deficit perspective \\
\hline $\begin{array}{l}\text { Context and } \\
\text { population }\end{array}$ & SMEs in Germany and their employees & Non SMEs, non SMEs in Germany \\
\hline Outcome & $\begin{array}{l}\text { Studies which do report on health- } \\
\text { oriented interventions }\end{array}$ & $\begin{array}{l}\text { Studies which do not report on health- } \\
\text { oriented interventions }\end{array}$ \\
\hline Study type & Primary research & $\begin{array}{l}\text { Book reviews, literature reviews, } \\
\text { opinion pieces, policy documents }\end{array}$ \\
\hline Methodology & $\begin{array}{l}\text { Studies containing findings which are } \\
\text { based on quantitative and/or } \\
\text { qualitative methods (data collection } \\
\text { and analysis) }\end{array}$ & $\begin{array}{l}\text { Studies which do not report on } \\
\text { methods applied (unclear } \\
\text { methodology) for data collection and } \\
\text { analysis }\end{array}$ \\
\hline
\end{tabular}

\subsection{Research ethics of the study}

An essential part of the research strategy is the consideration of its ethical aspects. I conducted the research ethics of my study following the University of 
Gloucestershire Research Ethics Handbook 'Research Ethics: A Handbook of Principles and Procedures' (University of Gloucestershire, 2008, p. 4).

In April 2018 my research project went through the 'Approval of Examination Arrangements' at the University of Gloucestershire. The University of Gloucestershire does not require an ethical approval of my research project.

However, since I include humans in my research, it is especially important to explain the ethical treatments as Gläser and Laudel (2009, p. 48) confirm "The treatment of research ethics within the area of social science research is indispensable." Thereby, ethics means "the application of values on concrete human behaviour" (Gordon, 1975, p.138). Further, I must take into account that research on humans creates a "plethora of possible ethical dilemmas", so that "researchers need to be cognisant of several ethical considerations" (Dantzker et al., 2016, p. 29). In view of these circumstances this study shows awareness regarding research ethic in the following points (Orb et al., 2001):

\section{$\checkmark$ Researcher and participant relationships \\ $\checkmark$ Interpretation of topic and data \\ $\checkmark$ Group conflicts \\ $\checkmark$ Finance of the study \\ $\checkmark$ Aim of the study}

Regarding the first point 'Researcher and participant relationship,' I choose top managers I know and choose top managers with leader experience and interest in the promotion of health. The employers choose the group of employees and they select them according to the same criteria 'open and interested in health promotion'. I explain to all participants how the selection comes about. The participants of the three SMEs do not know each other. The participants of the third focus group consist of family members and friends. All members of the third focus group have experience as employees, managing directors and department 
heads in the field of WHP and are interested in interpreting the data of my research from their point of view and discussing it in the group.

A further bias could be the interpretation of topic and interpretation of participants. That means I find matching answers in statements of participants or the participants which give answers which I want to hear. For preventing this bias, I try to ask for advice with the background of my coaching experience, so that statements cannot be misinterpreted. Besides, I behave as neutrally as possible, so that the participants answer honestly and do not simply give the desired answers.

Regarding the third point 'Group conflict' I have experience as a coach to deal with group conflicts such as 'hostility' or any other problems in the group. With the leading role as a moderator, I aim to prevent that no conflicts occur. All individuals have a right to a 'safe' environment in which they are allowed to speak and be listened to. Therefore, I create the conditions that several people do not speak at the same time but after each other. I leave no room in the discussion for gossip and step in if participants speak badly about individuals. However, as an experienced coach I counter balance any form of bias with convincing communication style and knowing about behavioural theories.

My research study is self-funded and has no third-party interest which might influence the research. The University of Applied Sciences (Fachhochschule des Mittelstands (FHM)) as my employer has no influence on my research and is not involved in it. The FHM provides only a room for the group discussions. The third focus discussion on the data takes place in private rooms. Therefore, it is a free research whose findings I would like to transfer into SMEs in EWL/Germany as a consultant.

This study interacts with its empirical investigations with top managers, their employees and with family and friends (third focus group) in their role as nonacademics and has to take the rights and interests of these participants seriously.

The significant principle for this research is to maintain the privacy, confidentiality, and anonymity of the research participants. The anonymity and privacy of 
research participants should be respected and personal information relating to participants should be kept confidential and secure (University of Gloucestershire, 2008, p. 5). In advance, with the help of a 'Participant Information Sheet' (PIS) I inform the focus- group participants about the expiration of the group discussion, about the possibility to leave the discussion at any time, about the use of the data (I store digital recordings are stored safely on a password-protected computer), about the anonymisation of the data and about the obligation to privacy as a participant. The PIS requires the participants to agree and sign (see example of PIS in appendix 9.8).

\subsection{Reflection of research philosophy}

When understanding research about well-being and health promotion of workforces in SMEs, it is important to consider different research paradigms and matters of ontology and epistemology. Because these parameters describe perceptions, beliefs, assumptions, the nature of reality, and veritable truth of that reality, they can influence the way in which the research is undertaken (Flowers, 2009). Therefore, it is necessary to examine the grounding of my research in these fundamentals of methodology and the research method. I give consideration to the personal development of a research philosophy that result in an appropriate research method and in productive outcomes.

\subsubsection{Ontological aspects of research}

Ontology is the philosophical study of the nature of existence or reality. "Key ontological questions concern whether or not there is a social reality that exists independently of human conceptions and interpretations and, closely related to this, whether there is a shared social reality or only multiple, context-specific ones." (Ormston et al., 2014, p. 4). 
The basic positions of ontology are objectivism and constructivism. Objectivism means having the characteristics of an object and therefore also an objective reality (Bryman et al., 2007). Hence objective truth has nothing to do with truth of individuals or subjects, and strictest objectivity is possible because the real world exists. An alternative ontological position is constructivism where knowledge and truth have to be constructed by individual subjects based on their individual experience of the world and their existence within it. Jonassen (1991) believes that reality is constructed based upon mental activity and on our unique set of experiences with the world and our belief about them. Transferred to business management, which is the research topic in this study, all aspects of a business process are socially constructed, because the promotion of health and well-being is a very individual experience as the individual subject has varying influencing factors on their health and well-being (age, illness, family, workload, resilience factors, etc.).

The topic of health promotion in SMEs should consider the structures, mechanisms, culture etc. of a company. It is about understanding and explaining the underlying mechanisms that are not normally observable (Bhaskar, 1998). For example, to explore how SMEs can empower employees, I include corporate value or corporate culture, for example. For this reason, the ontological position of critical realism fits well with my study because critical realism considers the reality in parts as socially constructed (and, also as independent of human beings) but observes also the mechanism and structure of organisations rather than experience of an individual. It can be considered as a fusion of objectivity and subjectivity.

The basic assumption of critical realism is the existence of a real world independent of our knowledge of it (Bhaskar 1998). Reality is conceived as being stratified in three domains; the real, the actual and the empirical. The real domain consists of structures of objects, both physical and social, with capacities for behaviour called mechanisms. These mechanisms may (or may not) trigger events 
in the domain of the actual. In the third layer these events may (or may not) be observed, in the empirical domain. Thus, structures are not deterministic; they enable and constrain events (Archer 1995; Sayer 2010).

A critical realist explores a phenomenon past the superficial appearance at the surface level of reality by asking more in-depth questions in order to gain a profounder understanding and meaning of reality: What works, for whom, in what respects, to what extent, in what contexts, and how (Marshall et al., 2014)? A claim of critical realism is to understand the world in all its complexity. With the ontological view as a critical realist, my study does justice to the underlying mechanism in SMEs. In addition, the critical realist view aims to bring about improvements to a system or structure, and therefore fits well with my practiceorientated DBA-study which seeks to provide practical tips on WHP for SMEs with its study findings. As Pawson (2004, p. 3) notices "the cornerstone of the realist project is a distinctive viewpoint on how intervention bring about change".

\subsubsection{Epistemological aspects of research}

Closely coupled with ontology and its consideration of what constitutes reality, epistemology considers views about what knowledge is and the sources and limits of knowledge. "A particularly central issue in this philosophical context is the question of whether or not the social world can and should be studied according to the same principles, procedures, and ethos as the natural science" (Bryman et al., 2007, p. 15). At large, epistemology defines how knowledge can be produced and argued for. It describes the criteria by which knowledge is possible and gives structure for what kind of knowledge is available and its limits (Eriksson et al., 2015, p. 14).

Historically, natural science develops a tradition with a neutral attempt to describe an un-biased picture of phenomena under consideration. In natural science, positivism is an epistemological position gathering value-free facts to generate 
hypotheses. Constructivist epistemology, on the other hand, is based on an existing reality as multiple mental constructions, in order to generate knowledge, gained by understanding the perceptions of people involved in the process. Interpretivism is the contrast of a positivistic epistemology (Bryman A. \& Bell E., 2011, p.16) also known as antipositivism in social science including Weber's 'Verstehen'-theory (Bryman et al., 2007, p. 16). Its focus is the study of the social world and of peoples' understanding of it. Critical realism is another philosophical position that considers human and natural science, and is therefore located between positivism and interpretivism, as the following figure shows:

Figure 4: The critical realist research position (own figure).

positivism

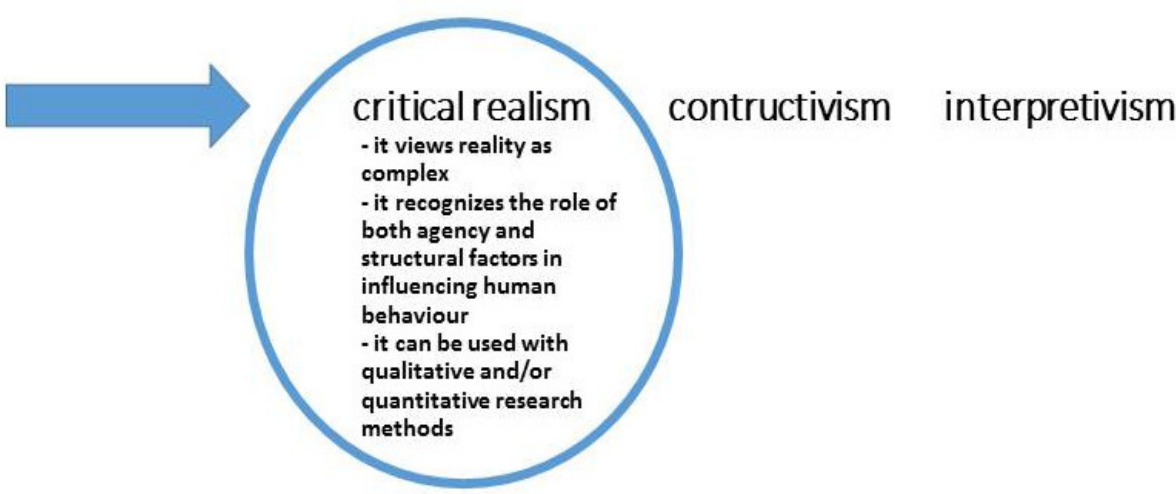

After classification of critical realism, it is interesting to look at the definition of critical realism. But defining critical realism is not an easy task because there is a pool of scholars that critical realists often draw upon (e.g. Archer 1995; Bhaskar 1975, 1979; Elder-Vass 2010; Gorski 2008, Lawson 1997; Porpora 2015; Sayer 2010). Critical realism is most closely associated with the early works of the philosopher Roy Bhaskar. It has been developed mostly in the social and health sciences and economics assumes that "social phenomena are made possible by the presence of humans but are deemed to be external to individuals and have existence and the power to constrain whether this is recognised by individuals or not." (Given, 2008, p. 168). Critical realism argues that such an individualism fails to recognise what is everywhere the reality - it is social relationships, whether 
enacted at a person-level (such as in social roles) or enacted at a collective level (such as enterprises), that comprise the categories of social science (Archer, 1995; Bhaskar, 1989).

"For the critical realist, social reality consists of much more than the linguistic, symbolic, and discursive resources and practice through which we come to describe, understand, and reflect on it; it also consists of entities, processes, relations and mechanism that are constituted and structured in particular ways [...]" (Buchanan et al., 2009, p. 434). The critical realist believes that phenomena create sensations which are open to misinterpretation because individual or subjective views vary in their interpretation of reality and the observer might misinterpret the actions (Bhaskar, 1975). To prevent misinterpretation critical realism focusses on explaining phenomena within a context. Related to research in businesses, it is about exploring dynamics and relationships between and in social groups to find the potential for change or improvement.

Further, Saunders (2011) argues that the critical realist's position - the social world is constantly changing - is much more in line with the purpose of business and management research which is often to understand the reasons for phenomena as a precursor to recommending change. This aspect also applies to my research which seeks to examine the phenomena 'health and well-being promotion of employees' in the context of SMEs, the region, etc. and to give recommendations that are sustainable and responsive to the ever-changing conditions of small and medium-sized businesses (SMBs).

Consequently, my study is based on the following definition of critical realism: Critical realists are seekers of practical methods for dealing with the complexities of social explanation (Pawson, 2004). 


\subsubsection{The use of critical realist paradigm for this study}

Using the paradigm of a critical realist corresponds to my ontological view that people experience their truths through social action and therefore truth is subjective. The paradigm of a critical realist considers the different mechanism and structures in SMEs, and therefore it fits with my research project in epistemological terms. To understand health and well-being promotion in SMEs it is necessary to consider the context of this kind of organisation and their features (company size, leadership level etc.) because the features can have an influence regarding health and well-being promotion of workforces.

The application of the critical realist approach shapes the research design, the study research questions, research method and the analysis and interpretation of the data. I could define the research strategy as a plan of how I will go about answering the study research questions and indicates the general orientation of how to conduct the research. Consequently, the research strategy is the methodological link between the philosophical background of the researcher and the subsequent choice of research methods to produce and analyse the necessary data (Denzin et al., 2011). The strategy of my study by collecting data via focusgroup discussions corresponds to the approach of critical realists. Critical realists tend to choose rigorous qualitative techniques (Bryman et al., 2007). That means no testing of hypotheses, but it allows knowledge to emerge. A critical realist explores a phenomenon by asking more in-depth questions in order to gain a deeper understanding and meaning of reality. A claim of critical realism is to understand the world in all its complexity. With the study method of focus-group discussions with top managers and employees, I can ask deep or further questions in order to get a comprehensive understanding of WHP in SMEs. This method also allows me to collect new insights and new knowledge to be collected which corresponds to the philosophical approach of critical realists.

Another argument in conducting a critical realist paradigm for my study is the formulation of my study research questions. As Reed (2009) recommends, 
research questions of the critical realist should be context specific (SMEs) and should also focus on underlying generative mechanism in SMEs. In this context, my study asks top managers and employees from one company (separated in two focus groups), so that my study is able to gain knowledge from different perspectives and therefore maybe gain knowledge about underlying mechanisms in SME. My study research questions (see chapter 1.7) aim to discover which initiatives promote workforce health and well-being and how initiatives can be implemented in SMEs. With that, I follow the critical realist goal to understand the complex interplay between structure (SMEs) and agency (top managers and employees). In other words, the critical realist-thinking researcher approaching reality as a social and contextual mental construction fits well with this study.

With the strong argument of an understanding of a deeper structure in SMEs I have selected the critical realist approach to be a good ontological foundation for Applied Thematic Analysis (ATA) (Guest et al., 2012). The way of using a mainstream technique like textual analysis, the data analysis is in line with the critical realist tradition (Reed, 2009). For the text analysis I conduct the ATA which seems to be one of the most established approaches to qualitative data analysis. ATA is frequently cited as the chosen approach in qualitative research studies. The enormous advantage of ATA is the wide scope of application as it provides theoretical flexibility (Guest et al., 2012, p. 18). This kind of text analysis aims to understand a specific phenomenon and can formalise the identification of themes and underlying mechanisms in this study (Guest et al., 2012).

The interpretation of the study findings also takes the underlying mechanisms or deeper structure in SMEs into account. As Pawson (2004) notices, realist evaluation tries to find out whether specific expected programme footprints can be identified in light of available data and data types. My study tries to find important mechanisms in SMEs with the study findings and take these into account in the data interpretation. 
Consequently, a critical realist position, in the context of this study, considers the research aim of this study to explore salutogenic health and well-being promotion of workforces comprehensively regarding the structures and mechanism of SMES in EWL/Germany.

\subsection{Research design}

This sub chapter sets out the concrete strategy of my research and displays the qualitative character of the study design.

\subsubsection{Strategy of the study}

I based my study approach on two processes to generate relevant data regarding health and well-being promotion of workforces: the systematic literature review (SLR) and the empirical research. I researched the relevant literature for my topic with clearly defined inclusion and exclusion criteria (see chapter 2.1.). It is about finding out what is out there and could be important for the search for initiatives to promote the health and well-being of workforces in SMEs in EWL/Germany. In order to find and apply relevant studies for my research, the literature search is based on a review question which is connected to the study research question, especially to the first research question. The review question serves as a focus and a framework in the phase of literature research. Regarding my practice-oriented DBA thesis and the aim to support SMEs regarding employees' health promotion in terms of salutogenesis, the following review question arises for the literature review: Which salutogenic factors support the promotion of health and well-being of the workforce in SMEs?

In the second step, I gather data through the focus-group discussion method. I select three SMEs from EWL/Germany with which I have capacity in a professional matter for the focus-group discussions. I form two groups in which one group consists of top management of the SMEs and the other group consists of employees of the top managers. Each focus group, guided by me as a moderator, 
discusses about 1.5 hours on the applicability of the nine WHP initiatives of the study by Bruch et al. (2013). I represent and explain each of the nine initiatives of the study by Bruch et al. (2013) are each represented and explained with a Power Point slide (see chapter 2.5). In addition, open questions regarding optimising of top manager awareness and about integrating of health promotion factors allow own ideas of participants.

As mentioned in chapter 1.7 , by consulting or rating the nine initiatives of the study by Bruch and the discussion of own ideas of participants triggered by the open questions I achieve relevant answers for the three study research questions through the focus groups. Therefore, the nine initiatives of the study by Bruch and the open questions are the research objects of my study which have a connection to the study research questions (see table 2). With this clear strategy of my study the focus-group method aims to get answers to the three study research questions (see chapter 1.7).

With the two processes of my study (literature review and empirical research), I can compare already existing knowledge with my empirical research data of the focus-group discussions in order to provide new insights to SMEs in EWL/Germany.

\subsubsection{Qualitative character of the study design}

Quantitative research requires that the researcher has a clear idea of what is being studied. It requires a process to be defined before beginning the research and for that process to be followed while gathering and analysing (Saunders, 2011). This study does the same for qualitative research. It involves the planning of the research project and the definition of what is being researched, because this study has a clear idea of who is going to be involved and of methods of data generation and data analysing. 
A host of definitions exist when searching for the definition of 'qualitative research'. Nkwi et al. (2001, p. 1) describe qualitative research with the exclusion of ordinal values: "Qualitative research involves any research that uses data that does not indicate ordinal values". While Gläser and Laudel (2009, p.27) characterise qualitative methods like those based on the interpretation of social issues, which result in a verbal description of these issues. A very simple, functional but appropriate definition is offered by Brosius, et al. (2001, p.21): "Qualitative methods describe a complex phenomenon in all its range." This study aims to understand the phenomenon of health and well-being promotion in SMEs. Thereby qualitative analysis, following Brosius et al. (2001) is preferred. Ordinal values are not to the fore but rather the context of social issues - the top managers' attitude to health and well-being promotion resulting in a verbal description of the research findings.

The main difference between qualitative and quantitative research is 'generating data' versus 'testing hypothesis'. Two different approaches to research exist which can be distinguished between the deductive and the inductive approach. Often inductive approaches are associated with qualitative research and deductive approaches are often associated with quantitative research, but rather the purpose of research is important. The deductive approach is aimed at testing a theory. Predictions have to be verified empirically. In philosophy, deduction is the science of conclusion. The inductive approach generates new theories based on evidence emerging from the data. The researcher tries to form scientific statements out of individual observations (Bryman et al., 2007, p. 26). Exceptions and creative combinations exist. The purpose of the research is to listen, to record, and to report about individual and social group experiences rather than scientific data evidence and to define what factors promote health and well-being of the workforce and to understand how SMEs in EWL/Germany can integrate health promoting factors. This approach is a descriptive objective of research and closer to the inductive approach, although the development of a theory is not the 
aim of this research. The limitation is the problem of generalisation. A scientific statement should be based on several but individually qualitative, inductive approaches. There is only a possibility to establish a useful base frame to understand the influence of the attitudes of top managers in SMEs in EWL/Germany as a contribution to health and well-being of the workforces. Because every participant of the focus-group discussions is an individual case, the research can only present a situated truth (Bryman et al., 2007). Therefore, my study requires a cross-sectional research design, because using this design entails the collection of data on more than one case. A study with a cross-sectional research design collects data at a single point in time in order to combine the data with two or more variables, which are then examined to detect patterns of association (Bryman et al., 2007). This research design is most suitable to explore salutogenic factors to support the promotion of health and well-being of the workforces in SMEs and to understand how SMEs can develop management awareness to keep their staff healthy.

Regarding the choice of words of the study research questions, I make important preliminary decisions regarding the choice of research method for data generation. Gläser and Laudel (2009) mention how the explanatory phenomenon described will have consequences to the method and how to attain the target explanation. With the method of focus groups this study produces a complete description of the phenomenon of health and well-being promotion in SMEs. The analysis goal is therefore exploratory; a theory-building aim is not planned because of the small sample, but it generates recommendations based on insider knowledge. On the other hand, it generates insider knowledge regarding WHP in SMEs in EWL/Germany.

To produce qualitative data effectively, focus-group discussions with several top managers and employees of SMEs in EWL/Germany have to be conducted to build up a concrete proposal and possible answers for each research question of this study. In choosing the method of focus-group discussions, the anticipated findings 
and their presentation (themes), the qualitative character of this study aims for clarity and transparency and wants to generate new practice-orientated knowledge of health and well-being promotion in SMEs. To answer the study research questions a mechanism orientated explanation strategy is required and the resultant understanding is the main emphasis for the research.

\subsection{Method of the study}

The focus- group method is a form of group interview in which:

- there are several participants,

- an emphasis in the questioning on a particular topic and

- the accent is upon interaction within the group and the joint construction of meaning (Bryman et al., 2007).

This method contains elements of two methods: the group interview, in which several people discuss a number of topics, and the focused interview in which interviewees are selected because they 'are known to have been involved in a particular situation' (administered to individual or groups) (Merton, 1967) and are asked about that involvement (Bryman et al., 2007). Hence, the focus-group method is more in line with the focussed interview because of the elements of interaction within groups as an area of interest. So, the focus interview is more focused than the group interview (Kitzinger, 1995).

Focus-group discussion is a technique that collects data through group interaction on a topic determined by the researcher (Morgan, 1996). This type of group interview is a way to gather together people from similar backgrounds or experiences to discuss a specific topic of interest (Krueger, 2014; Morgan, 1988). If the participants of focus groups have similar backgrounds, the researcher will more quickly find discussion topics based on common experiences. In recent years, SMEs in Germany have learned through various initiatives such as the 
'Proactive SMEs' (called 'Offensive Mittelstand' in Germany) (initiated i.e. by the German Federal Government) that there are many advantages in networking and exchanging ideas, experiences etc. within SMEs. Thus, the participating SMEs of my study could see benefits in exchanging WHP topics with other SMEs in the group discussions.

As Bryman (2007) notices focus-group discussions emphasize a specific topic that is explored in depth. Therefore, this method is suitable for my research because I would like to get a deeper insight into SMEs regarding health and well-being promotion of workforces. Besides, I also saw the early use of the focus-group technique as a way of helping individuals to define problems and work together to identify potential solutions (Hutt, 1979). As the participating SMEs have little or no experience in health promotion, especially with a focus on well-being, the group dynamics could lead to joint concepts and solutions within the group.

The strength of this method relies on allowing the participants to agree or disagree, with each other so that it provides an insight into how a group thinks about an issue, about the range of opinion and ideas, and the inconsistencies and variations that exist in a particular community in terms of beliefs and their experiences and practices (Stewart et al., 1990). The possibility of top managers and employees to agree and disagree within the group, offers a greater range of opinions and views of participants so that I can gain deeper insight and information about health promoting factors in SMEs in EWL/Germany. On the other hand, there is the potential risk that the group could make potentially wrong or unwise decisions because of group pressures which then could lead to a deterioration of "mental efficiency, reality testing, and moral judgement" (Janis, 1972, p. 9). Focus groups can be affected by 'groupthink' (Janis, 1972) which is a phenomenon that occurs in groups and the members of the group could ignore alternatives and tend to take irrational actions. A focus group is especially 
vulnerable to groupthink when its members are similar in background, but the moderator has the role and responsibility to manage this risk if it occurs.

In contrast to the methods of questionnaires or interviews, focus groups can be used to examine not only what people think, but how they think and why they think this way (Kitzinger, 1995). Group processes can help people to explore and clarify their views. In addition, the participants of my study can learn from other SMEs through the exchange in focus groups. By incorporating the opinion of SMEs through focus groups the participants have the feeling of taken seriously and have the feeling to play an active part in WHP in SMEs. Further, the sense of togetherness which is important attribute for SMEs is strengthened by the focus group method in my study. Some researchers have noted that group discussions can generate more critical comments than interviews (Watts et al., 1987), and group conflicts may also arise e.g. hostility or group pressure as mentioned before. I prevent critical comments and group conflicts by informing the participants well about the purpose, exploration etc. of the focus-group discussion. With my experiences as a business coach I learned how to deal with group conflicts. For example, with the method of 'solution-oriented coaching' (Freeman et al., 2007) I am confident in asking questions about conflict solution. Further, with my convincing communication style and experiences as a moderator of groups I tried to counteract conflicts early.

It is important to use group discussions for data production in this study because with the attitude of a critical realist I explore the deeper mechanisms in SMEs although this method is not typical for critical realist's approaches. It is necessary to ask top managers why they evaluate and assess the presented WHP initiatives in this way. The deeper questioning with the focus-group method about attitudes, values etc. help to collect data that serve my research goal to find out: What salutogenic factors can be generated by top management to support the promotion of health and well-being of the workforce? Consequently, the method of group discussions is more likely to suit the study research questions and it is 
hoped to be answered successfully this way rather than other methods of data production.

However, I have to prepare carefully the focus-group discussions through identifying the main objectives of the group, developing key questions, developing an agenda, and planning how to record the session. Also I have to note finding suitable discussion participants and getting the ideal number of participants (between six and eight) has to be noted (Stewart et al., 1990). Krueger et al. (2014) summarises more important points to bear in mind when facilitating focus groups: Careful wording of the key questions, maintaining a neutral attitude and appearance and summarising the session to reflect the opinions evenly and fairly. The following sections of this chapter cover the topics of Krueger et al. (2014) and Stewart (1990) and explain and justify the method of focus-group discussions for my study in more detail.

Three SMEs of EWL/Germany meeting the selection criteria (see chapter 2.5.1) are chosen in order to receive the required information for this research study. Out of 11 potential participants, five are top managers and six are employees taking part in the focus-group discussions. The study conducts two focus groups. The first one is the group of top managers of SMEs and the second one consists of the employees of the top managers of the first focus group. In conducting the same focus-group discussions for top managers and employees, I want to make sure that I receive important information from both sides given under identical conditions. Furthermore, I conduct two focus groups with their two perspectives (top managers and employees) in order to generate comparable data and to gain a more holistic understanding.

For the focus groups I develop a scenario is developed in order to create and discuss opinions in a relaxed and open atmosphere for the research participants. The scenario method helps me as a researcher to make sense of, and address, complex and uncertain contexts (Ramirez et al., 2015). That means for my study 
that I can explore the complex theme of health and well-being promotion in SMEs regarding the complex health promotion context like the influence of top managers and due to structures in SMEs. This way of data gathering can generate 'interesting research' because it is both rigorous and actionable. I prepare a scenario with nine health promotion topics and open questions in the German language, the mother tongue of all participants of the two focus-group discussions. I guide the focus groups by myself as the moderator. I introduce the nine health topics of the study by Bruch et al. (2013), and I also help the group to participate in a lively and natural discussion amongst themselves (see chapter 2.5.2).

Before introducing the scenario to the focus groups, I give a brief introduction which includes an overview of the background and purpose of the study, personal introductions as well as confidentiality and anonymity, etc. I explain my role as moderator of the discussion (see chapter 2.5.2) and I inform the group that I am responsible for the frame and the time of the focus group setting. I present each of the nine topics of the study by Bruch et al. (2013) on a Power Point slide (see appendix 9.11). To introduce the nine topics in a way which is interesting and open for discussions, I create a fictional top manager named 'Ben Müller' and a fictional enterprise named 'WE GO':

Figure 5: The slide of the Power Point Presentation of focus-group discussion to introduce 'Ben Müller'.

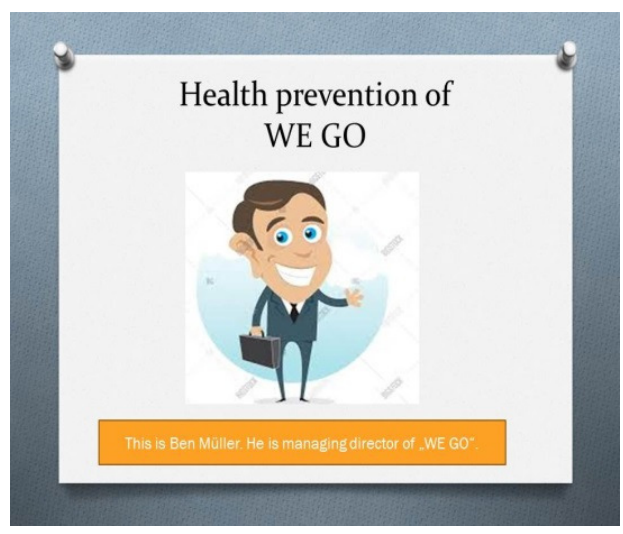


The following figure shows one topic of the study by Bruch et al. (2013) 'Top management should be a pioneer' (see appendix 9.11) with examples explaining the topic.

Figure 6: Slide of the Power Point Presentation of focus-group discussion to introduce the topic 'top management as a pioneer'.

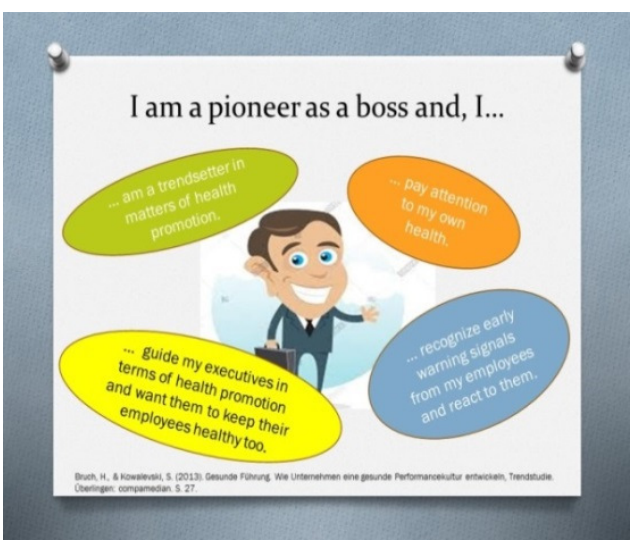

With the help of the creative or animating Power Point slides, the focus-group participants learn about the topics or initiatives and can find out what it means to implement them in companies. After presenting a topic or measure, I ask open questions about the practicability of the introduced measure and about experiences that the participants already may have regarding the presented measure. After each measure, I ask the same three questions which are intended to ascertain the participants' experiences, views and attitudes towards health and well-being promotion (see figure 7):

Figure 7: Example slide to introduce the kind of questions after representing each topic.

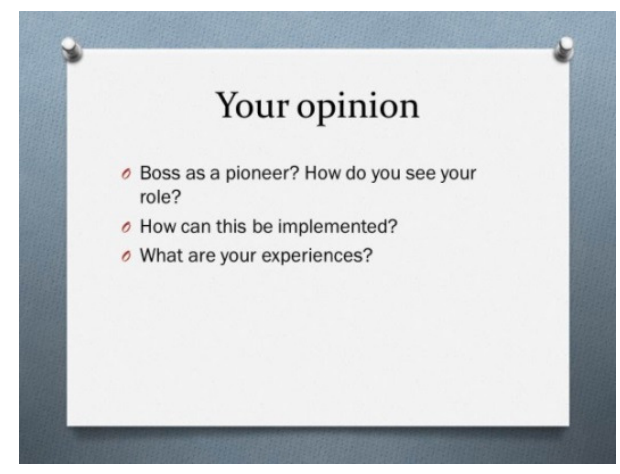


In summary, I present nine Power Point slides with the nine topics of the study by Bruch et al. (2013) in the focus groups. As already outlined in chapter 2, I link the nine topics of the study by Bruch et al. (2013) to the SoC factors of the salutogenesis (see figure 2) and are therefore I present them in the focus groups as salutogenic WHP initiatives. The aim is to interrogate the initiatives on practicability in SMEs and at the same time find out, whether the nine initiatives primarily strengthen the feeling of comprehensibility, manageability or the feeling of meaningfulness of workforces. As mentioned before, the combination of the SoC theory by Antonovsky with the study by Bruch et al. (2013) make it possible to meet the current trend in salutogenic WHP of employees.

At the end of each focus group two additional open questions are asked because I want to know which of the nine topics are rated of high value, importance or relevance by the focus-group participants (see figure 8 ).

Figure 8: Slide of the Power Point Presentation of focus-group discussion to present the first open question.

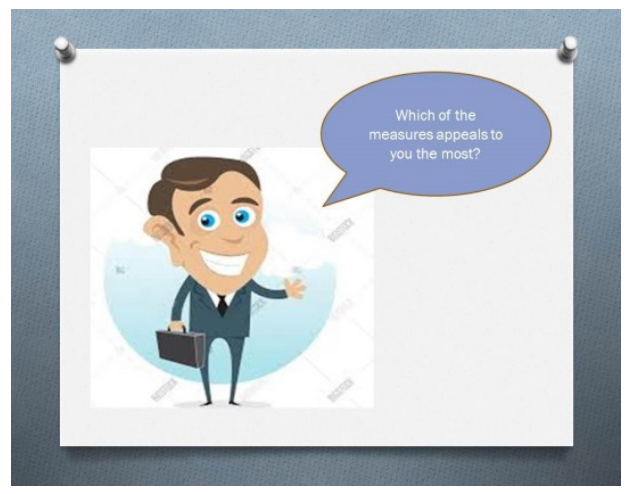

With the first open question 'Which of the measures appeals to you the most?' I get a final assessment of the participants' views and attitudes and can be sure that nothing is forgotten during the focus-group discussion. By formulating the second open question ('I would like to continually expand and improve the health promotion of my employees. Are there any ideas?) I get more ideas of health and well-being promotion in SMEs. 
Figure 9: Slide of the Power Point Presentation of focus-group discussion to present the second open question.

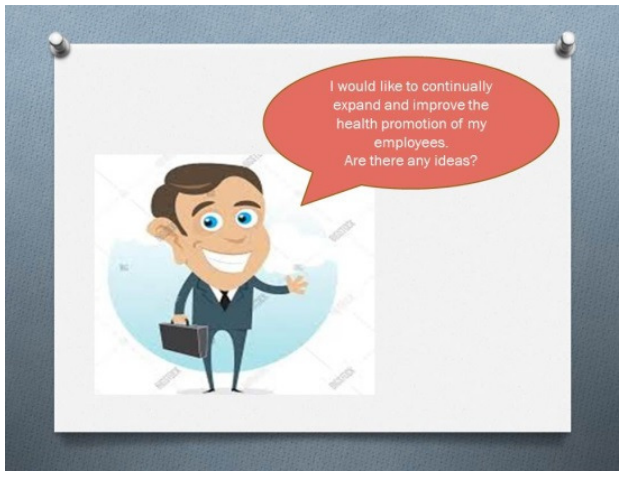

As mentioned before, I take responsibility for the frame and the time of the focus groups (Morgan, 1996). I should also follow up on statements to receive profound information and opinions of the participants. The qualifications of an experienced business coach help me with regard to asking the right questions to get important answers or information from the study. As well as being a professional business coach, observing and reacting at the right time is what matters in focus-group discussions (Krueger, 2014).

Summarising, I prefer the focus-group discussions with scenario methodology moderated by myself for this study because this method enables exploring attitudes, values etc. towards promoting health and well-being of workforces in SMEs. Thus, I bring together findings from the focus-group discussions to result in answering the three study research questions. I monitor the quality of the focusgroup discussions concerning the validity and reliability. I select the ATA for focusgroup analysis, as being important to the description of a phenomenon (see chapter 2.6). A detailed analysis plan is developed and the focus-group logistic and recording is planned. As a necessary basis I identify the themes for textual qualitative analysis and describe them in a codebook (see appendix 9.9) to prepare the analysis with NVivo as the selected Qualitative Date Analysis Software for this research project (see chapter 2.6). 


\subsubsection{Selection of focus-group participants}

The selection of participants impacts on the quality of information the researcher obtains (Bryman et al., 2007). In this study the selection of participants is purposive because the participants have particular features or characteristics which enable detailed exploration and understanding of the central themes and questions which I wish to study (Easterby-Smith et al., 2012). I summarise the characteristics of participants of the study as criteria in the following table:

Table 4: Selection criteria of research participants.

\begin{tabular}{|l|l|}
\hline Topic relevant criteria & Selection criteria \\
\hline 1 Type of top manager & $\begin{array}{l}\text { Top manager with leadership experience and interested in the } \\
\text { topic of health and well-being promotion }\end{array}$ \\
\hline 2 Type of enterprise size & SME \\
\hline 3 Region of SMEs & $\begin{array}{l}\text { East-Westphalia Lippe (EWL), North Rhine Westphalia, } \\
\text { Germany }\end{array}$ \\
\hline 4 Type of employee & $\begin{array}{l}\text { Long-term employees of participating SMEs and interested in } \\
\text { the topic of health and well-being promotion }\end{array}$ \\
\hline
\end{tabular}

I select the SMEs and their top managers based on companies to which I have access or connections in a professional capacity. I chose only enterprises where the top managers have leadership experience and those who have expressed that they are interested in WHP and are willing to discuss the theme with other SMEs. The type of company is not of concern here. It does not matter what type of business the company undertakes, because Health and Safety regulations imposed by law are not considered in this study. What is of importance and relevance to the research study is their attitude towards health and well-being promotion. As Marczak et al. (1991) recommend I select enterprises with their participants for the focus groups who are unfamiliar with each other.

The question about who possesses the necessary knowledge to answer the study research question is imperative, because to gain the required information I need 
to ask various participants (Bryman et al., 2007) about health and well-being promotion factors which have different roles (employees or employer) and different experiences. Consequently, I select participants from top management and employees from each SME for the focus-group discussions. I anticipate getting additional information about WHP initiatives from employees because they are likely to look at health and well-being promotion factors from a different perspective. For example, top managers may look differently on the topic as they are the ones who need to provide money for WHP. Employees, on the other hand, may not consider the company's cost for health promotion. Employees can supplement themes of their top manager or discuss entirely new themes regarding health and well-being promotion. In order to receive the required information for this research, the top managers are important as well as their employees.

Out of 12 potential focus-group members who fitted the selection criteria (see table 5), I accept 12 requests for group discussions and I conduct 11 (one failed to attend).

Table 5: Information about members of focus-group discussions.

\begin{tabular}{|l|l|l|}
\hline Description & & Quantity \\
\hline Potential participants of the focus groups & & 12 \\
\hline Group discussion request accepted (one failed to attend) & & 11 \\
\hline$\rightarrow$ Focus-group discussions in summary & & 2 \\
\hline Top managers & & 5 \\
\hline Employees & & 6 \\
\hline$\rightarrow$ Recorded and transcribed & & app. 3.5 hours \\
\hline
\end{tabular}

The gender of identified focus-group members is unequally distributed. Three of the 11 participants are female, while eight are male. They are between 28 and 65 years of age and are all native German speakers.

All the chosen top managers have long-term experience in leading businesses of SMEs. Another reason to choose these leaders is that 1 know them in a 
professional capacity and therefore I have access to their companies. In addition, they are open minded generally and interested in WHP for SMEs.

Two of the five top managers own and lead SMEs in EWL/Germany. Three are salaried general or senior managers. Two SMEs are placed in the manufacturing industry and the other is placed in the tradesman business. The participating SMES have from 12 to 220 employees. They have no integrated health management system, but some have a little experience in WHP of employees.

The second group consists of six employees (two employees from each SME), which are chosen by the three top managers. I left the selection to the top managers because I am aiming for voluntary participation of those who are willing to participate and therefore hopefully to contribute the most helpful information. In addition, the top managers themselves are better able to assess which of their employees are open for group discussions. Nonetheless, this aspect of selfselection is taken into consideration by making sure in the group discussions that the employees are really interested in the topic and thus fulfilling the requirements.

\subsubsection{My role as a moderator}

As the moderator I do not interfere or intervene in the discussions so not to influence the participants too much. My role as moderator is to keep the discussion flowing and to clarify any questions. I have to negotiate the time frame and ensure the participants stay on topic and do not veer off to discussions of other topics which are not relevant to the study. Importantly, I have to check the voluntary participation and consensus to take part in the research and focus-group discussions, as well as information about confidentiality and their options to withdraw from the research study at any time. I am careful regarding my influence during the focus-group discussion and try to be natural in the body language (not giving value to what participants say i.e. by smiling too much or agreeing too much). I have to pay attention to be independent and neutral as a moderator. My 
coaching degree and coaching-experiences as a coach helps me in this, in which I have learned not to act in an evaluative way and to be non-influencing in my questioning. Besides, I have to take care to stay out of the discussion, because I am not a participant and should only guide the discussions. I also need to pay attention to group dynamics and processes (i.e. talking too much and being over powering in the group, or people getting hostile, etc.) and be responsible for balance in the group. I must be prepared for the phenomenon of 'group thinking' and be able to deal with it because of my coaching-experiences in business. I need to ensure it does not end up in a 'blaming' and 'moaning' session by being a positive example with an optimistic and hopeful behaviour and attitude. I should also ensure a pleasant and confidential situation.

\subsubsection{Duration and size of focus-group discussions}

I plan for the focus-group discussions to last between 60 and 120 minutes. The reason for that duration is not to tire the participants out and overstretch their concentration span or enthusiasm and willingness to participate freely in the research study. The optimum time per focus-group discussions is not longer than two hours but often top managers asked for a shorter time slot (Krueger et al., 2014). I need to consider and accept these limitations or requests. Therefore, I decide to conduct the focus-group discussions within a time frame of approximately 60 to 80 minutes in a breakfast or brunch type set up.

Little agreement is found in the literature on optimal focus-group size. Groups of eight members generate more ideas than groups of four members, eight member groups do not produce twice as many ideas as two four member groups did (Tang et al., 1995). Whereas, Slater (1958) recommends five members to be optimum for discussing a human relations problems. This study plans for conducting group sizes between five or six members, depending on availability of the participants. 


\subsubsection{Interviewing in the German language}

The focus group language is German because all participants are German speakers. The German transcripts are not translated to English, because the problem of misunderstanding and distortion is too great. The option of translation is not an ideal solution because of the risk to misinterpret the information. As a compromise, I extract the key information in German and I summarise and partially translate only the German citations. Relevant passages are translated into English as closely as possible to the original.

\subsubsection{Focus-group recording and transcribing}

The analysis of focus-group discussions is an important part within the research method because the researcher on his or her own affects the data in significant ways. Within qualitative approaches the gathering of information is influenced by the researcher's context and bias so the analysis cannot be disconnected from the gathering of information (Myers, 2013; Silverman, 2013).

One of the key challenges of the analysis and interpretation of qualitative data in a research project is the huge amount of data that can emerge (Bryman et al., 2011; Flick, 2012; Myers, 2013). As mentioned in subchapter 3.5.3 the duration of the focus-group discussions in this research project last from 80 minutes (for the first group) and up to 75 minutes (for the second group). I audio-record and transcribe all focus groups. I transcribe the interviews within the week following each focusgroup discussion. The audio data end up in around two-and-a-half to three hours of audio data, 62 DIN A4 pages of printed text and 23,704 words.

I answer the question how to transcribe the focus groups is answered by the suggestion of Saunders et al. (2011) and Bryman et al. (2011), who recommend to transcribe the audio data entirely and shortly after each interview. I decide to employ an external service provider to type up the transcripts from both focus groups (and the reflection group). The same person fully transcribes all the 
interviews. Saunders et al. (2011, p.486) point out two main problems when paying a touch typist:

- 'You will not be familiarising yourself with the data as you are not transcribing it yourself.'

- 'The transcription will still require careful checking as errors can creep in.'

I solve these potential problems through a detailed control of the transcript with the original audio data, but I expect the quality of the transcripts to be very good and only need a few changes with typing mistakes and unfamiliar names and terms. This procedure allows me the control of the transcripts shortly within a week after each focus group or reflection group.

\subsubsection{Consideration regarding reliability and validity}

There is a debate within qualitative research circles that researchers do not aim to achieve the same reliability or validity as in quantitative research (Lavrakas, 2008). Due to these problems I decided to provide the outcomes of this study by the means of Guba and Lincoln (1994) based on their theory of trustworthiness and authenticity in order to meet the validity and reliability as far as it goes.

Trustworthiness means in qualitative research to take care about credibility (do the researchers study at a reputable university etc.?), transferability (do the findings have an application elsewhere etc.?), dependability (has all the data been properly recorded etc.?) and rigour (are the research steps as objective as possible etc.?). To fulfil these conditions the study by Bruch et al. (2013) could help as the basis of group discussion. But I must consider the special region in Germany of EWL, and the special size of the companies (SMEs), in which my research takes place must be considered regarding transfer to other businesses sizes and businesses regions.

I carry out both discussions in the same way. Further, I pay attention to recording technology and test the technology in advance. In addition, I am supported by an 
assistant who works well in technical matters and looks after the technical things during the discussions. I adapt a rigorous research process (see chapter 2.5 ) and plan and implement the focus-group discussions according to the same schema. I am aware that these are two different groups (employees and employers) and therefore it can never be identical. For example, the time pressure of top managers could play a role, so that they cannot muster as much patience for the discussion as their employees.

According to Bryman (2007) rigorous research is research that applies the appropriate tools to meet the stated objectives of the investigation. Consequently, I make sure that the study research questions, the research objectives and the research strategy (see chapter 2.4.1, table 2 ) precisely match to one another.

In addition, I check the data with the suggestion of the Theory of Authenticity by Guba and Lincoln (1994). Authenticity means in qualitative research to take care regarding fairness (does the data represent all opinions? etc.), ontological authenticity (do the findings help participants to better understand their situation? etc.), educative authenticity (do the findings help participants to better understand the perspectives of others? etc.), catalytic authenticity (has the research caused participants to try to change their situation? etc.) and tactical authenticity (has the research empowered participants to change their situation?). Since I am working as a business coach, I aim to ensure in my role as a moderator that the discussions of the participants are fair, gain new knowledge and help to understand the subject for the participants. I can support the participants to change or revise opinions during the discussions.

With this alternative approach by Guba and Lincoln (1994) I consider reliability and validity of qualitative methods. Therefore, the data of my study result in trustworthy findings. 


\subsection{Analysis of the study}

The focus-group discussion analysis of my study is a qualitative data analysis because it interprets text out of audio recordings and is placed in the upper left square of Bernard's (1996) figure:

Figure 10: Qualitative and Quantitative Data Analysis (adapted from Bernard, 1996).

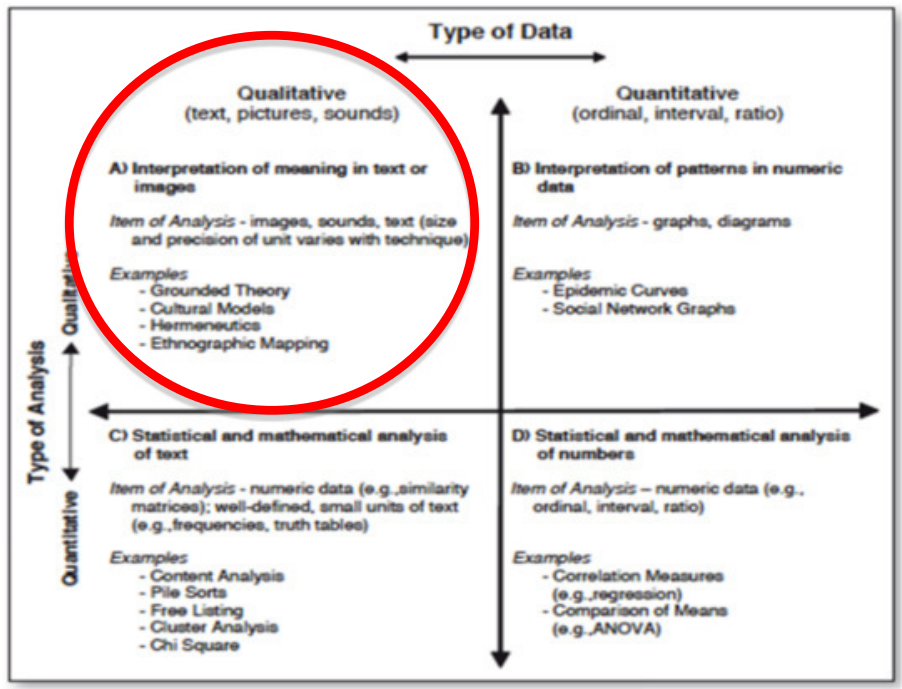

In this research, I intend to define which salutogenic factors support the promotion of health and well-being of workforces in SMEs. As discussed later, I conduct the investigation of this definition by focus-group discussions. The analytic purpose is exploratory and content driven. I will describe and argue the decision of ATA in the following subchapter.

\subsubsection{Applied Thematic Analysis selected for focus-group discussions analysis}

After reviewing the literature about qualitative research or focus-group analysis for example (Berg et al., 2004; Braun et al., 2006; Morgan, 1996; Ryan et al., 2010; Stewart et al., 1990) it became clear that the ATA (Guest et al. 2012) best describes the method necessary in this research project. The ATA can involve 
multiple analytical techniques and it seems to be one of the most established approaches to qualitative data analysis. It provides theoretical flexibility (Guest et al., 2012) and therefore it could explain the wide scope of application.

The ATA is not new. It is based on commonly employed inductive thematic analysis and shares many features with Grounded Theory and phenomenology. Grounded Theory for example uses a systematic comparative technique, aimed at building theory, whereas phenomenology focuses on subjective human experience (Guest, et al., 2012, p. 17). While Qualitative Content Analysis arises from the analysis of huge amounts of text originally, the information is categorised and frequency analysed, and often the comparison of cases is to the fore (Gläser \& Laudel, 2009, p. 197ff).

With the help of the ATA, key themes can be identified which can be transformed or transcribed into codes and aggregated in a codebook (Guest et al., 2012, p. 17). Further, ATA analysis is a search for themes that emerge as being important to the description of the phenomenon (Daly et al., 1997) and provides an answer to the research questions which I address. The theoretic flexibility allows different ways in which thematic analysis can be approached:

- An inductive way - coding and theme development are directed by the content of the data;

- A deductive way - coding and theme development are directed by existing concepts or ideas;

- A semantic way - coding and theme development reflect the explicit content of the data;

- A latent way - coding and theme development report concepts and assumptions underpinning the data;

- A realist or essentialist way - focuses on reporting an assumed reality evident in the data;

- A constructionist way - focuses on looking at how a certain reality is created by the data (Madill et al., 2000).

The separation of the different ways is not always found that rigidly in reality. I base this research project on critical realist ontology. I implement the critical 
realist way, which focusses on how reality looks and is determined by my perception, is implemented. The research project prefers the inductive way, coding and theme development directed by the content of the data. The inductive way allows me as a researcher to stay open for new health promotion themes.

Within ATA the researcher has the choice between three analytic approaches, the exploratory, explanatory and confirmatory analysis. The decision to choose the exploratory analysis for this project is because the "emphasis is on what emerges from the interaction between researcher and respondent" and within the focusgroup participants (Guest, et al., 2012, p.35-39). These interactions are important and drive the development of codes and the identification of themes. An explanatory analysis, which uses a combination of deductive and inductive methods, is helpful in the analysis of question number ten 'Which of the measures appeals to you the most?'

After considering the questions to start the analysis of the research project, it is important to give the analysis structure. Braun and Clarke (2006) describe a sixphase process of analysing, which follows the approach to ATA:

Figure 11: Six phase process of thematic analysis (own figure, content adapted from Braun \& Clarke (2006)).

Familiarisation with the data
Coding

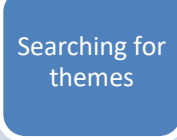
Reviewing
themes

Defining

and

naming themes
Writing up

The process of the analysis begins with the 'familiarisation with the data', which means reading and re-reading the data to become familiar with its content. This is followed by the 'Coding' phase which means to identify important information of 
the entire dataset that might be relevant to answering the study research questions. The topics that are discussed again and again at different points in the group discussion, or measures that are discussed intensively, are coded. In addition, I include topics as codes that are rated as important by the participants. I turn the substantial information which is extracted into a suitable format and process it separately from the original text with the help of the software NVivo.

The next phase starts with the identification and 'Searching for themes' and involves collating data relevant to each of the nine themes by Bruch et al. (2013), so that I can work with the data. The 'Reviewing themes' phase involves checking the themes against the dataset to determine that they include necessary information to answer the study research questions. Further, I conduct a detailed analysis of each theme in the phase 'Defining and naming themes'. In this phase I write down the name for each theme. The final phase 'Writing up' means to define the most significant themes and weave together the analysis of each theme by contextualising the analysis to the study research questions and to existing literature.

Although these six phases are sequential, and each build on the previous, analysis is typically a recursive process. It moves back and forth between different phases. However, it is not rigid, and with more experience and smaller datasets, the analytic process can blur some of these phases together (Braun \& Clarke, 2006).

After the detailed overview of the ATA as the chosen analysis method, I develop an analysis plan for my research project.

\subsubsection{Analysis plan}

Developing an effective analysis plan is the key to answering the study research questions which are based on the study rationale. This can be reached by the 
identification of necessary activities that need to take place in order to reach the desired research outcome (Guest et al., 2012, p.46).

Four key elements of the analysis of qualitative data provide an overview of the process (MacQueen et al., 1999, p. 27-39).

I. Characteristics of the sources where answers to research question are sought.

The sources of assessment are the results gained from the two focus-group discussions with knowledge about WHP. The participants have to be assessed by nine factors (see chapter 2.5.1) to gain answers to the research questions.

II. Primary information collected from the sources (for example how to form the questions of the focus groups).

The primary information is collected from the transcript of personally conducted focus-group discussions (the manner of transcription is described in the chapter 2.5.5).

III. Information generated to assist in the interpretation of the primary information (e.g. codes, code definitions, coding notes, descriptive summaries of source information such as word frequencies, code frequencies, code co-occurrence matrices).

Codes are generated to assist in the interpretation of the primary information (see chapter 2.6.3). The study produces fourteen themes (gathered from the focus groups), which are classed with codes and described in the codebook (see appendix 9.9).

\section{Information about the characteristics of the coder.}

The coder in this research project is myself, Julia Pönighaus, in the role of a doctoral student and a research assistant at a University of Applied Sciences (FHM) with the background of an educational scientist and as well a corporate consultant and a business coach for SMEs (see chapter 1.2). It can be assumed that the 
scholarly interest is to the fore, because the research project is not based on the university's projects or interests but rather based on personal interest. My research project is self-funded, and the outcome of findings has no influence on the interests of third parties. The interest of a high-quality doctoral thesis, with contribution to the knowledge base, is a good basis for efficient and reliable findings.

In addition to the point 'Information generated to assist in the interpretation of the primary information' of MacQueen et al. (1999), I illustrate unexpected topics and evaluate them using the ATA.

\subsubsection{Process for extracting codes and themes from focus groups}

In this research project I make use of ATA whilst using text segmentation as a tool by coding relevant data extracts. Guest et al. (2012) recommend a small data set consisting of a few focus groups, and that developing an explicit segmentation is not necessary. However, in this research project the use of text segmentation as a tool is applied in order not to lose a theme and to generate an overview for me within this project. The data are the transcripts, as the textual representation of the focus groups, while the themes are the unit of meaning that is to be observed.

Figure 12: The coding process with basic terms in textual qualitative analysis (own figure).

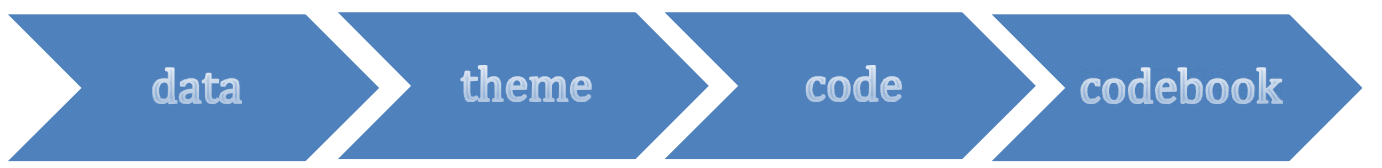

The coding process is "obviously one of the key phases in the whole process of qualitative data analysis" (Bryman et al., 2007, p. 608). It is a fundamental task in most qualitative projects and an essential method within ATA. It involves gathering all the material about a particular theme or topic into nodes, or knots, 
for further exploration. Within this research project I allocate the references of 62 pages of transcription to different codes.

Within ATA an effective qualitative codebook is helpful, or absolutely necessary, if the dataset is huge. "Developing a codebook is a discrete analysis step where the observed meaning in the text is systematically sorted into categories [...]". (Guest et al., 2012, p.52). In my study the codebook (see appendix 9.9) is supportive because it gives me, as well as the reader, a clear overview to enable them to follow the way analysis is conducted. The following figure shows an example of a code (see all codes in the codebook in the appendix 9.9):

Table 6: Example of a code of the codebook.

Code: 1 Boss_Pion Ini

Brief Definition: Boss as pioneer and initiator

Definition \& representation of the scenario (opinion of Mr. Müller): I am trendsetter in the matter of health promotion. I pay attention to my own health. I would like to influence others to promote leading also healthy lives. I recognize early warning signals from my employees and react to them. Question within focus-group guideline: Q1. Mr. Müller is a pioneer as a boss: How is that with you in your enterprise? How can this be implemented? What are your experiences?

RQ 1 \& 3: Contributes to define salutogenic factors that can be generated by top management to support the promotion of health and well-being of workforce and contributes to understand how SMEs in EWL/Germany integrate health promoting and well-being factors.

Example: „So assuming a certain role model in the subject of health is certainly advantageous in my opinion [...]."

I often cannot match the information from the focus groups clearly to one code; sometimes crossing with other codes occurs. In case of double informationl assign two codes.

Nevertheless, I can match the codes principally to one or two of the three study research questions and I can also check for relevance to salutogenesis, relevance to factors of the study by Bruch et al. (2013), and to the relevance to the review 
findings (see chapter 4.2.1).

\subsubsection{Qualitative Data Analysis Software - NVivo}

The number and features of software programmes for qualitative data analysis grows continuously and offers several capabilities, "they have converged to the point of being superb text base managers with text- and code-retrieve functions that facilitate theory and conceptual network building - and much more."(Guest et al., 2012, p. 218).

The study size and complexity of the research project decides about the necessity of a Qualitative Data Analysis Software (QDAS). I have to take account several factors like the size of data, the complexity, the analysis goal and the size of study team to decide upon the use of a QDAS (Guest et al., 2012, p. 223, 224). Guest et al. (2012) mention that projects including more than 20 in-depth interviews, more than two analysts, moving from descriptive to explanatory goals and moderate complexity may require a Qualitative Data Analysis Software.

Software use within this research project is optional considering the relatively small size of the study team and the analysis goal. I work on my own and not within a study team of two or more analysts. I have two supervisors supporting me in technical and methodological terms.

The analysis goal is exploratory because the aim is to understand how SMEs in EWL/Germany integrate health and well-being factors. On the other hand, the complexity of study is complex, because it is not easy to keep track of two focusgroup discussions, with 62 pages of transcribed material, 3.5 hours audio material and 14 codes identified. The size of data sets is, with two focus groups, not as much as 20 interviews, but much material comes together so that a QDAS is organisationally useful. NVivo is the selected qualitative data analysis software for analysing the transcripts within this research project (see table 7). 
Table 7: Established NVivo project for this research project.

\begin{tabular}{|c|c|c|c|c|c|}
\hline 'noten & Suche nach & Suc & Suche in & $\cdot$ & Q6 Chr \\
\hline Knoten & \multicolumn{5}{|c|}{ Q6 Challenging and supporting } \\
\hline Q41 Top management as pioneers & Name & & Quellen & Refere & renzen \\
\hline Q14 Weitere Ideen \& Maßnahmen & Abwech & & 1 & & 1 \\
\hline (4) Q11 Was nehmen Manager für die Praxi & Älteren & & 1 & & 3 \\
\hline 14. Q2 Healthy Self Management & Arbeitsi & & 1 & & 2 \\
\hline 14. Q3 Freedom with a safety net & Gesund & & 1 & & 1 \\
\hline (1) Q4 Meaningful Work & Unterne & & 1 & & 5 \\
\hline \multicolumn{6}{|l|}{ 145 Value } \\
\hline \multicolumn{6}{|l|}{ (2) Q6 Challenging and supporting } \\
\hline \multicolumn{6}{|l|}{ 18. Q7 Holistic health management } \\
\hline \multicolumn{6}{|l|}{ 18. Q8 Health consumption } \\
\hline 14. Q9 Psychological immune system & & & & & \\
\hline
\end{tabular}

\subsection{Summary of methodology and method chapter}

The methodology of this research is based on the critical realist attitude because that fits well with my own view as a researcher and it also fits with my complex research field of SMEs with their different mechanisms and structures. As adapted for critical realist research, the data is qualitative in order to generate opinions or suggestions based on individual experiences. The chosen methods follow qualitative focus-group discussions as the ideal type of methodological approach. The purpose of this methodology is to explore in the first step relevant data for my topic with help of the literature review, and in the second step to gather data with the focus-group method in order to syndicate the data with existing knowledge, and further to generate new knowledge regarding health and well-being promotion in SMEs. Further, I represent ethical issues. As preparation for the systematic literature review, I clearly define the research rationale and research aim with the inclusion and exclusion criteria of the study. This provides the prerequisite for a systematic literature review of the next chapter. With the decision of reviewing the literature in a systematic way, the study aims to minimise bias through exhaustive literature searches (Tranfield et al., 2003). The next chapter 'Literature Review' aims to find salutogenic factors that promote the health and the well-being of workforces in SMEs. 


\section{Literature review}

As mentioned before, my study aims to help SMEs with the knowledge of salutogenesis in their efforts to maintain and promote employees' health and well-being to meet their future challenges. With a concrete review question, I would like to find measures that enable SMEs to keep their employees healthy or to promote their health and well-being. This chapter introduces at first the origin and development of systematic literature review (SLR) and its significance for organisational and management research. In order to present a comprehensive overview of the research environment, a definition and a classification of the term 'health and well-being promotion in respect of salutogenesis' follows. Moreover, I will define the term 'small and medium-sized enterprise' (SME). Afterwards, the chapter 3.2 'Methodology of systematic literature review' introduces the process and the method and explains why the SLR is expedient for my research project. It introduces the review question that underlies the SLR. After that I will present the literature scoping (chapter 3.3) and searching of different sources to find relevant data and knowledge. Based on the inclusion and exclusion criteria (see chapter 2.1) the critical examination of the inclusion criteria follows (chapter 3.4). The chapter 'Quality Assessment' (chapter 3.5) completes the view of external studies. The focus then turns to the 'Integrating and synthesising qualitative research' (chapter 3.6) to gain an overview of the studies focussing on well-being and health of workforces in SMEs and to bring research closer to the use of health and wellbeing promotion initiatives for SMEs. The last section explores the health and wellbeing promoting initiatives in terms of salutogenesis through the strongest themes emerging from the synthesis and shows the findings.

\subsection{Origin and development of systematic literature review technique}

Since the late 1980s, the interest in evidence-based approaches focussed primarily in the medical and health research area, because practice was based on poor- 
quality evaluations of the literature, that sometimes caused inappropriate medical recommendations (Tranfield et al., 2003). Researchers were required by government agencies and other institutions to apply a more systematic and scientific approach to the practice of reviewing the literature. In other words, it needed a more systematic and reproducible method for identifying, evaluating and interpreting the existing body of recorded work produced by researchers, scholars and practitioners (Fink, 2019). This corresponds to Centre for Reviews and Dissemination (CRD) observation which notice that a systematic review has been argued to bring replicable, scientific, and transparent approach, which seeks to minimise bias through exhaustive literature searches (Khan et al., 2001). In addition, "major improvements in review techniques over the last fifteen years or so have helped to raise the profile of systematic reviews as an important research methodology" (Denyer et al., 2009, p. 672).

Systematic literature reviews (SLR) differ from narrative reviews by adopting a more rigorous and well-defined review process (Cronin et al., 2008), by following protocols that include comprehensive searches for all potentially significant studies (Cook et al., 1997), and by its replicability, offering an audit trail of all the reviewers research steps in a scientific and transparent manner (Tranfield et al., 2003). Booth et al. (2011) claim that the systematic review technique requires reviewers to summarise all existing information about a phenomenon, and with a thorough critical evaluation of existing research, new insights can be found by synthesising previously unconnected ideas (Hart, 2001). Petticrew (2008) noticed that SLR has value in collecting and synthesising existing evidence across a wide range of settings (including the social sciences) and empirical methods. With the different methods and their application in different sciences, criticism is raised that SLR in social science does not often meet the quality criteria (validity, reliability) such as SLR in medicine or health sciences. The critique of SLR in social sciences focuses on how such evidence is used to inform policy, practice, and future research (Buchanan et al., 2009). Denyer et al. (2009) recommend that researchers in social science (including management and organisational research) 
have to be explain how and in what ways the notion of evidence based management might benefit the field through improved quality and rigour, by defining a bespoke and fit-for-purpose format for review. It is possible for researchers in management or organisational studies to have a significant opportunity to evaluate and learn from other areas that have developed an evidence-based approach that uses systematic reviews as the key method (Denyer et al., 2009).

\subsection{Methodology of systematic literature review}

Traditionally, systematic reviews pre-describe that studies are expected to confirm to a set of principles as well as adopting specific methods to identify, select, and critically appraise relevant primary research (Denyer et al., 2009). But Tranfield et al. (2003) voice concern, that the adoption of traditional principles of SLR coming from medicine research is undesirable for use in the field of management and organisation studies. Therefore, my study follows the recommendations of Denyer et al. (2009) who introduced four SLR principles which can be applied in management and organisation studies:

- Transparency regarding the processes and methods employed in the review

- Inclusivity regarding the quality of information sources

- Explanatory reviewing, or narrative reviewing, as an alternative approach to metaanalysis

- Heuristic outputs or designing solutions to problems instead of presenting the truth

The SLR of my study does justice to the first key principles of Denyer et al. (2009) 'Transparency' by providing the reader with information about the scope and boundaries of the review in the first step. I review the most important decisions (creating the review question, inclusion and exclusion criteria, quality appraisal) and findings regarding the processes and methods of my literature review (instead of an extra protocol chapter). Further, the study findings are presented in such a way that there are clear links between the evidence found and my conclusion and 
recommendations. The SLR takes also the quality of sources into account by using a quality assessment (see chapter 3.5). The third key principles 'Explanatory' also fulfil my SLR by using a narrative review synthesis approach instead of metaanalysis because I aim to find practical solutions for SMEs instead of finding the truth. The SLR methodology fits with the exploratory design of my thesis and corresponds well with the critical realist perspective of my study because my study does justice to the underlying mechanism in SMEs.

The SLR of this study can be divided into five main steps applying the principles for management and organisational studies by Denyer et al. (2009). The first step is to formulate a well-formulated review question which gives the review a concrete focus and guides the review by defining which studies are included (Counsell, 1997). My study complies with the recommendation of Tranfield et al. (2003) to check or refine the review question after scoping study if necessary.

It is important to clarify the purpose and the aim of the study before formulating a question for the SLR. There are different frameworks for review methodology coming from, e.g. health science or social science called SPIDER, PICOC, PICO or $\mathrm{CIMO}$ etc. With the help of this frameworks the researcher can check the review question whether the question sufficiently takes into account, e.g. the context of the research. For my study I choose the PICOC method because it considers the population (workforces), the context (SMEs) and the focus on well-being promotion in comparison to only physical health promotion (comparison). The CIMO method was developed specifically for critical realist approaches by Denyer and Tranfield (2009), drawing on Pawson's work on evidence-based practice from a realist perspective (Briner et al., 2009). Nevertheless, the CIMO model does not focus on population (workforces) and on comparison (promotion of well-being versus only physical health promotion) and therefore CIMO is not used in this study.

However, PICOC helps me to determine whether the findings of a study are generalisable and applicable to the organisational context of SMEs even when the population, outcome of interest and SMEs characteristics are so different from 
those in the study that its results may be difficult to apply (CEBMa, 2019). The following table shows the PICOC method for my review question:

Table 8: PICOC method of review question.

\begin{tabular}{|c|c|c|}
\hline Population & Who? & Workforce \\
\hline Intervention & What or how? & $\begin{array}{l}\text { Health and well-being promotion in } \\
\text { terms of salutogenesis }\end{array}$ \\
\hline Comparison & Compared to what? & $\begin{array}{l}\text { - Health promotion addressing only } \\
\text { physical side } \\
\text { - Cause elimination by health promotion }\end{array}$ \\
\hline Outcome & $\begin{array}{l}\text { What are you trying to } \\
\text { accomplish/improve/change? }\end{array}$ & Find out what employees get healthy \\
\hline Context & In what kind of organisation? & SMES \\
\hline
\end{tabular}

In the next step I check whether the terms used for the PICOC method (see table 9) are used as components of the review question. The review question is already presented in the previous chapter are checked with the PICOC method in the following table:

Table 9: Check of review question by PICOC method.

\begin{tabular}{|l|l|}
\hline Terms identified by PICOC method & Marked components of review question \\
\hline Workforce & $\begin{array}{l}\text { Which salutogenic factors support the promotion } \\
\text { of health and well-being of the workforce in SMEs? }\end{array}$ \\
\hline $\begin{array}{l}\text { Health and well-being promotion in terms } \\
\text { of salutogenesis }\end{array}$ & $\begin{array}{l}\text { Which salutogenic factors support the promotion } \\
\text { of health and well-being of the workforce in SMEs? }\end{array}$ \\
\hline $\begin{array}{l}\text { - Health promotion addressing only physical } \\
\text { - Cause elimination by health promotion }\end{array}$ & $\begin{array}{l}\text { Which salutogenic factors support the promotion } \\
\text { of health and well-being of the workforce in SMEs? }\end{array}$ \\
\hline Find out what workforces get healthy & $\begin{array}{l}\text { Which salutogenic factors support the promotion } \\
\text { of health and well-being of the workforce in SMEs? }\end{array}$ \\
\hline SMEs & $\begin{array}{l}\text { Which salutogenic factors support the promotion } \\
\text { of health and well-being of the workforce in SMEs? }\end{array}$ \\
\hline
\end{tabular}


The second step is named 'Locating studies' and aims to locate, select, and appraise as much as possible of the research relevant to the particular review question (Buchanan et al., 2009). The review of my study uses several methods, including searching electronic databases, hand searching known journals, recommendations from experts, and cross-referencing, as recommended by Buchanan et al. (2009). The third step focusses on 'study selection and evaluation'. To fulfil the requirement for transparency of review processes, my SLR uses explicit selection criteria to assess the relevance of each study to see if it gives answers to my review question. I use a 'quality assessment check' (see chapter 3.5) to evaluate studies because the different subfields of management have different perspectives on research quality (Buchanan et al., 2009). The SLR reports on the study and its limitations and justifies also the exclusion of studies which do not fulfil the quality. The fourth step is called 'analysis and synthesis'. "The aim of the analysis is to break down individual studies into constituent parts and describes how each relates to each other" (Denyer et al., 2009, p. 685). This step allows me to make an association between the themes identified in individual studies and create new concepts from that (Hart, 2001). Denyer et al. (2009) recommend as the last step to report on empirical research and to provide a statement of the problem and the review question. This step also specifies precisely what is known and unknown about questions addressed in the review. My SLR represents in the conclusion chapter (3.9) a summary of the review, the limitations of the study, recommendations for SMEs in EWL/Germany, and future research needs.

In the following chapters I perform the five review steps (presented above) recommended by Denyer et al. (2009) and give detailed explanations and justifications. 


\subsection{Literature scoping}

For the starting of a literature review Tranfield et al. (2003) proposes a scoping study to assess the size of the literature and evidence sources or research and to delimit the subject area by finding studies of different subject areas. The scoping study included the search for existing reviews and primary studies suitable to the review's objectives. This scoping includes searching for existing research, undertaken reviews, or primary studies about initiatives of SMEs to promote employee's health and well-being. The Center for Evidence Based Management (CEBMa) (2019) recommends to conduct in the two most relevant databases for the field of management: ABI/INFORM Global and Business Source Elite or Premier from EBSCO. Depending on my review question I also need to search in databases that are aimed at neighbouring disciplines such as psychology (PsyclNFO), healthcare (PubMed), or nursing (CHINAHL). In addition, research articles can be found through Google Scholar or Google Books. To gain an initial overview for my topic I choose electronic databases e.g. Business Source Complete, PyscARTICLES (see table 13). By examining several databases, I want to check in which databases the relevant studies are located.

I carried out the search on 31st May 2019 using the relevant German and English search keywords (see table 10). I did not accomplish a hand-searching. As recommended by the CEBMa, I have read various studies and systematic reviews about my topic and become familiar with research terms in my field of research. My keywords should reflect the different aspects of my thesis, such as health promotion at the workplace, promotion of the physical and mental health of employees, SME as a company size, and salutogenesis as a health theory. The keywords of my scoping study should also be a good basis to be able to answer the review question. I define keywords in order to carry out a pre-testing to check whether keywords generate relevant studies (CEBMa, 2019). 
Table 10: Keywords for literature scoping.

\begin{tabular}{|l|l|l|}
\hline No. & Keyword German & Keyword English \\
\hline 1 & Betriebliche Gesundheitsförderung & Workplace health promotion (WHP) \\
\hline 2 & Maßnahmen der Gesundheitsförderung & Health-promotion programmes \\
\hline 3 & Mitarbeiterwohlbefinden & Employee well-being \\
\hline 4 & Salutogenese & Salutogenesis \\
\hline 5 & $\begin{array}{l}\text { Kleine- und mittelständische Unternehmen } \\
\text { (KMUs) }\end{array}$ & $\begin{array}{l}\text { Small- and medium-sized enterprises } \\
\text { (SMEs) }\end{array}$ \\
\hline
\end{tabular}

The pre-test is a helpful exercise to test if the keywords (see table 10) provided relevant results for WHP in SMEs. I have learned that the keywords are best to search in abstracts to get suitable results for answering the review question. The pre-test revealed that the combination of the keyword 'salutogenesis' with the keyword 'SMEs' yielded no returns. I decide to choose the keyword 'salutogenesis' as a term option in abstracts in the next step.

\section{Search outline}

For the systematic literature review I search different sources to find relevant data and knowledge. I exclude non research-based literature. The research question and search terms are orientated by the Centre for Evidence Based Management (CEBMa) (2019) as mentioned before. I base the search strategy of the study on the question 'Which salutogenic factors support the promotion of health and wellbeing of the workforce in SMEs?' and I include the following sources:

\section{Databases incl. search engines}

As mentioned before, I conduct the search on core bibliographic databases via WordCat and EBSCO and search engines called Business Source Complete, PyscARTICLES, Psychology and Behavioral Sciences Collection, PsycINFO, SocINDEX, CINAHL, WorldCat.org, Emerald Group Publishing Limited, SpringerLink, 
Wiley Online Library, Nexis Uni, ProceedingFirst, WorldCat, ArticelFirst, Electronic Collections Online, ABI/INFORM Global, Electronic Books, OAlster, Medline, Science Direct, SAGE Journals, Open Access to Theses and Dissertations, Google Scholar and Google Books. The five keywords (see table 11) are the basis for search terms for databases and search engines. I convert them in terms that reflect both the German and English language. Furthermore, I use synonyms (employees or workforce) of search terms to find relevant studies. The databases provide the researcher with several options to specify the search (Center for Evidence Based Management (CEBMa), 2019). I make use of so-called Boolean operators (AND, OR and NOT), which allow me to combine search terms in different combinations.

Table 11: Search terms for databases and search engines.

\begin{tabular}{|c|c|}
\hline 1 & Betriebliche Gesundheitsförderung* OR Workplace health promotion*OR WHP \\
\hline & OR \\
\hline \multirow[t]{2}{*}{2} & $\begin{array}{l}\text { Maßnahmen der Gesundheitsförderung* OR Health-promotion initiatives* OR Health- } \\
\text { promotion activities OR Health-promotion initiatives* OR Health-promotion programmes }\end{array}$ \\
\hline & OR \\
\hline \multirow[t]{2}{*}{3} & $\begin{array}{l}\text { Mitarbeiterwohlbefinden* OR Employee well-being * OR Employee wellbeing* OR employee } \\
\text { well-being OR workforce well-being * OR Workforce wellbeing* OR workforce well-being }\end{array}$ \\
\hline & OR \\
\hline \multirow[t]{2}{*}{4} & Salutogenesis* OR Sense of Coherence* \\
\hline & OR \\
\hline 5 & $\begin{array}{l}\text { Kleine- und mittelständische Unternehmen* OR KMUs* OR Small and medium-sized } \\
\text { enterprises* OR SMEs* }\end{array}$ \\
\hline
\end{tabular}

In the first step, I find the sources by using one of the five terms (see table 11 above) via abstract. I also set the term 'workplace health promotion' (WHP) as a term for a keyword in data base search. 
Figure 13: Screenshot of English terms.

\begin{tabular}{|c|c|c|c|c|}
\hline & & \multicolumn{2}{|c|}{ Index durchsuchen } & \multirow{2}{*}{$\begin{array}{l}\text { Suchbegriff } \\
\text { workplace health promotion* }\end{array}$} \\
\hline & & Stichwort & v & \\
\hline \multicolumn{2}{|l|}{ Operator } & \multicolumn{2}{|c|}{ Index durchsuchen } & Suchbegriff \\
\hline UND & v & Abstract & v & workplace health promotion* \\
\hline \multicolumn{2}{|l|}{ Operator } & \multicolumn{2}{|c|}{ Index durchsuchen } & Suchbegriff \\
\hline ODER & 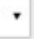 & Abstract & v & health promotion programmes* \\
\hline \multicolumn{2}{|l|}{ Operator } & \multicolumn{2}{|c|}{ Index durchsuchen } & Suchbegriff \\
\hline ODER & • & Abstract & v & employee well-being* \\
\hline \multicolumn{2}{|l|}{ Operator } & \multicolumn{2}{|c|}{ Index durchsuchen } & Suchbegriff \\
\hline ODER & v & Abstract & v & salutogenesis* \\
\hline \multicolumn{2}{|l|}{ Operator } & \multicolumn{2}{|c|}{ Index durchsuchen } & Suchbegriff \\
\hline ODER & . & Abstract & • & SMEs* \\
\hline
\end{tabular}

In the second step I change the search for the term 'SMEs'. The term 'SMEs' should appear as a condition in the abstract now. With this second search method I filter out many non-relevant studies focusing only on big companies etc. This reduces the hits of 120,688 returns to 72 returns (see table 12 below). Then, I read the titles and abstracts of the 72 studies to see if they really show relevance to my review question. The examination showes that I can continue to include the 72 studies because they show relevance as the following table shows:

Table 12: Quantitative search results and numbers extracted.

\begin{tabular}{|c|c|c|c|c|}
\hline Databases/engines & $\begin{array}{l}\text { Date } \\
\text { searched }\end{array}$ & Hits & Title & $\begin{array}{l}\text { Extract } \\
\text { No. }\end{array}$ \\
\hline PubMed & 31.05.2019 & 10 & 7 & 3 \\
\hline $\begin{array}{l}\text { Business Source Complete, PyscARTICLES, } \\
\text { Psychology and Behavioural Sciences Collection, } \\
\text { PsycINFO, SocINDEX, CINAHL } \\
\text { (all via EBSCO) }\end{array}$ & 31.05 .2019 & 1,007 & 26 & 1,411 \\
\hline
\end{tabular}




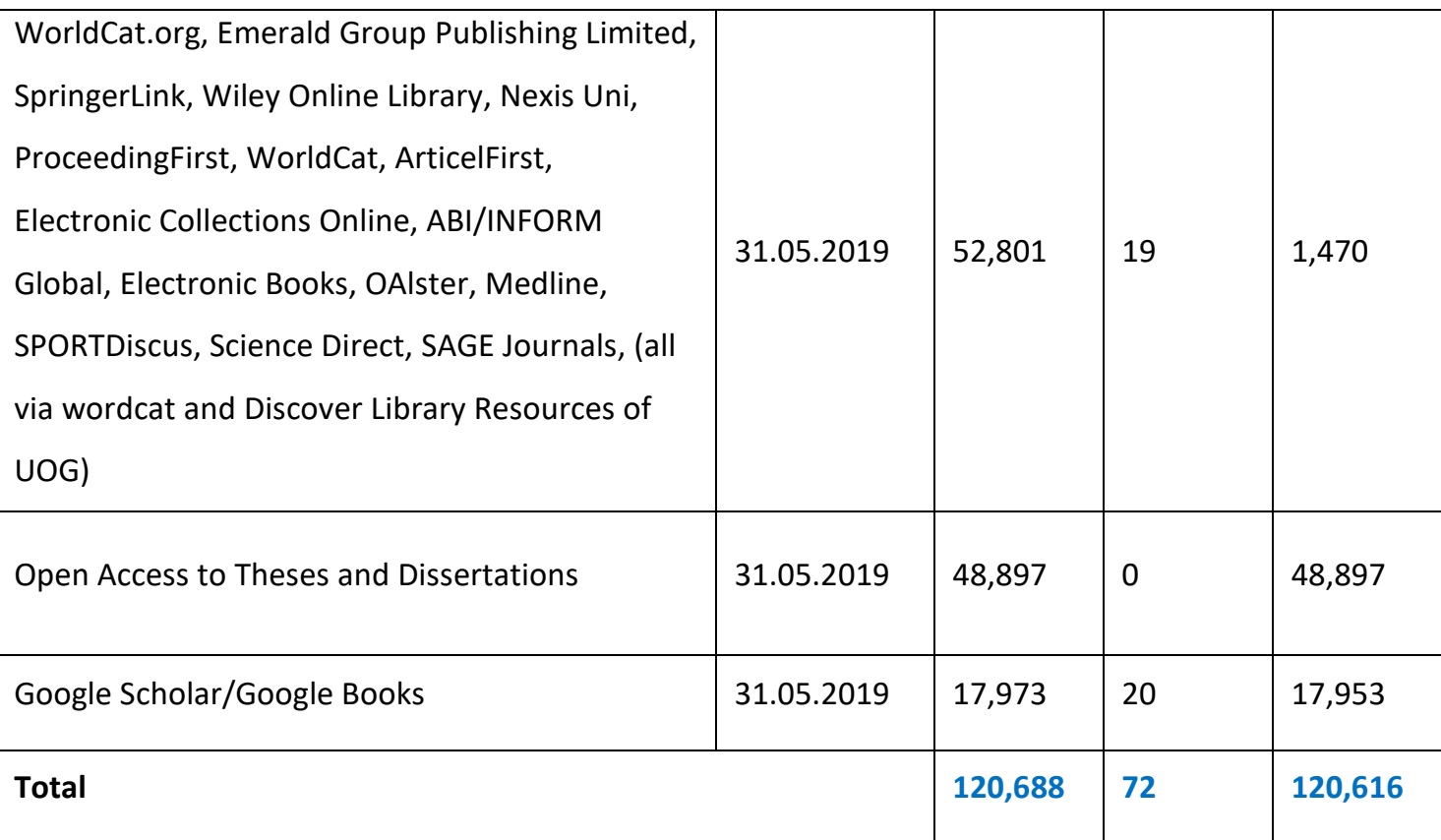

This first scoping study demonstrates that there are many sources that deal with WHP but only a few studies focusing on SMEs. There is also quantitative research on WHP initiatives, but qualitative data on how SMEs can integrate them, and which initiatives are suitable for SMEs are rare. Further, I consider the psychological or mental aspects of WHP initiatives in SMEs below average. The first scoping study shows that the theory of salutogenesis and the health and wellbeing promotion in SMEs are unrelated.

A primary result of the initial examination of literature revealed a lack of a systematic review addressing health and the well-being promotion of workforces in terms of salutogenesis in SMEs. Therefore, the scoping exercise demonstrated that a systematic review of qualitative research of salutogenic initiatives of SMEs to promote the health and well-being of their employees had not been conducted. The following systematic review collects the relevant sources of WHP initiatives in terms of salutogenesis for SMEs to promote the well-being and the health of employees.

As suggested by Tranfield et al. (2003), this scoping study in public internet databases accomplishes a systematic review by searching in published and 
unpublished literature. Such grey literature is any published and unpublished material that is not normally identifiable through conventional searches and includes for example: conference proceedings, newspaper, research in progress, reports, sources of institutions or working groups (Hart, 2001). Examples are: internet sources of institutions and initiatives, key journals and books which provide me with a current and practice-oriented knowledge about health and well-being promotion in the workplace and which give me an overview of the research field, the practical experiences of businesses, use of salutogenesis in the economy, and future needs of businesses regarding employees' health and wellbeing. The following sources are considered in the SLR:

\section{$\underline{\text { Internet sources }}$}

I conduct internet searches of websites of institutions and initiatives which provide practice-oriented knowledge about health and well-being promotion in the workplace. The following institutions provide current data of WHP and also guidelines or instructions for businesses regarding implementation of WHP measures: University of Bielefeld - Health Science (https://www.unibielefeld.de/gesundhw/ forschungsprofil/ index.html), CENTER FOR INNOVATION IN THE HEALTH INDUSTRY East-Westphalia Lippe (ZIG) (https://www.zig-owl.de), Bertelsmann Foundation (www.bertelsmann-stiftung.de), Federal Institute for Occupational Safety and Health (BAuA) (https://www.baua.de), Federal Ministry of Health (https://www.bundesgesundheitsministerium.de/ themen/praevention /betriebliche-gesundheitsfoerderung/weiterfuehrende-informationen.html),

World Health Organization Regional Office for Europe (http://www.euro.who.int/de), European Health Management Association (http://www.ehma.org), Initiative of the Federal Ministry of Health 'Business Enterprise Health' (www.unternehmen-unternehmengesundheit.de), Top Job Trendstudy (www.topjob.de), Initiative New quality of work (INQA) (https://www.inqa.de/DE/Startseite/start_node.html), Statutory health insurance (GKV) (www.gkv-spitzenverband.de), Initiative Health and Work (iga) 
(https://www.iga-info.de), Chartered Institute of Personnel and Development (CIPD) (https://www.cipd.co.uk), CORPORATE HEALTH INITIATIVE (https://www.corporate-health-netzwerk.de), Great Place to Work ${ }^{\circledR}$ (https://www.greatplacetowork.de), Future Institute GmbH (https://www.zukunftsinstitut.de), Scientific Institute of the AOK (WIdO) (https://www.wido.de), Institute for Employment and Employability (IBE) (http://www.ibe-ludwigshafen.de), Research institute for the future (IZA) (https://www.iza.org/de), Institute for Health Prevention (IFGP) (https://www.ifgp.de), European Agency for Safety and Health at Work (EU-OSHA) (https://osha.europa.eu/de), The German Network for Workplace Health Promotion (DNBGF) (http://www.dnbgf.de).

\section{$\underline{\text { Keyjournals }}$}

I electronically search the following journals: Oxford Journal of Medicine \& Health, Social Sciences, Health Promotion International, American Journal of Health Promotion, ASR - American Sociological Review, AJS - American Journal of Sociology, International Journal of well-being, International Journal of Qualitative Studies on health \& well-being, Qualitative Health Research, Sociology Journal, LSE - British Journal of Sociology, Absenteeism Report (Fehlzeiten-Report), Journal of Management, Journal of Health Psychology, Zeitschrift für Personalführung, Journal of Health Sciences, European Health Management Association (2005 Annual Conference), Work \& Stress, International Journal of Human Resource Management, International Journal of Stress Management, Journal of Business and Psychology, Journal of Psychosomatic Research and Business psychology, Journal of Occupational Health Psychology, Journal of Small Business and Enterprise Development.

\section{Books}

I search in title and abstract relevant edited books which reported on health and well-being promotion in terms of salutogenesis in SMEs. I include only evidencebased evaluation of WHP initiatives in terms of salutogenesis in SMEs focusing on health and well-being of employees after full text reading. 


\section{Citations}

I follow up references citations (via reading titles and abstracts) from relevant journal articles, literature reviews, books and reports for further potential research studies.

\section{Search results}

I search the data base search and engines search after reading titles and abstracts elicited in summary 72 returns (see table 12 ). By searching in titles and abstracts in journals, books, reports I still have found 35 additional returns. By checking references citations within reports, SLR and books I have found 91 further relevant studies after title and abstract reading. With the help of the 'snowball method' in google scholar (by clicking on the 'related articles' link I can snowball to other articles that may be relevant and search for related terms) I still find 135 relevant returns after title and abstract reading. Overall, the scoping study has yielded 333 returns which I fully read and analyse with the help of the inclusion and exclusion criteria (see chapter 3.4).

\subsection{Inclusion and exclusion criteria}

Following the methods of systematic reviewing I check the 333 returns of the scoping study if they give answers to the review question. Hence, I define clear inclusion and exclusion criteria for the further search of relevant studies of the 333 returns which I explain and justify in the following section.

\section{Locations}

The theory of salutogenesis has an influence on the concepts and on the public health scene, especially in the USA, Scandinavia, Australia (Blättner, 2015). It also has an impact on health promotion in the work context in Europe (Mittelmark et al., 2016). The model of the salutogenesis is applicable mainly in the social, psychology and medical areas in Europe (Homfeldt et al., 1997). The idea of the salutogenic model has its beginning in the USA. Aaron Antonovsky publishes the 
main and most important books in the USA. Because of these points I limit the location of the literature in Europe, USA and Australia.

\section{Language}

I use studies in German and English language as I speak these languages. Research results with other languages are not considered.

\section{Time frame}

The German Occupational Safety and Health law of 1996 plays an important foundation for the implementation of health promotion in companies in Germany (Bruder et al., 2010) because it functions as a kind of 'Basic Law'. With the Luxembourg Declaration, a declaration on WHP was written in 1997 by the European Union, thus laying down a uniform idea of what health care criteria means in the workplace. Hence, the time frame of my literature research is based on a German legal adjustment and secondly on a declaration that is aimed at improving the quality of $\mathrm{CHM}$ in Europe. Both measures can lead to more attention in the research and practice of CHM from 1996 onwards, which explains the timeframe (1996-2019) of my study.

\section{$\underline{\text { Subjects }}$}

In order to clearly express the dissertation's focus, I include only studies reporting on workplace health and well-being promotion.

\section{$\underline{\text { Relevance }}$}

The model of salutogenesis offers my study an ideal theoretical framework for the research field of health and well-being promotion in SMEs. The physical and mental aspects of health often coincide and play together (Bruecker et al., 2012), yet the study places a special focus on the well-being of employees because of its connection to salutogenesis. As stated in the first chapter, it takes a rethinking of $\mathrm{CHM}$ in companies from purely physical WHP such as fitness and back exercises to the promotion of mental health. The framework of salutogenesis gives my study a resource-orientated view on WHP, and therefore follows a trend in workplace health promotion (WHP). 


\section{Context and population}

The study takes place in SMEs in EWL/Germany and focus on employees of this special businesses size. My research follows the quantitative definition of SMEs of the 'Institute for SME Research' (IfM) (see chapter 1.3) because this definition takes into account different company sizes. It involves companies with a small size, with a medium size and those which start to grow up to a big company (based on the number of employees and annual turnover). The Institute for SME Research (IfM) (2019) defines companies with up to nine employees and/or less than $€ 1$ million annual turnover as small and those with ten to 499 employees or an annual turnover of $€ 1$ million to less than $€ 50$ million as medium-sized companies. The totality of SMEs thus comprises all companies with less than 500 employees or $€ 50$ million annual turnover.

\section{Outcome}

In order to create a theoretical framework of WHP it is necessary to include studies which report on processes and results of employee health and well-being. I exclude studies which only focus on pathogenic factors are excluded. The study thus has a positive and resource-oriented focus in the field of CHM in SMEs in EWL as mentioned before. Therefore, I search for studies with enablers of successful WHP via thematic analysis.

\section{Study type}

Based on Clarke and Oxman's study (as cited in Tranfield, et al., 2003) only primary studies can contribute to synthesizing evidence from various studies in the SMEs sector. As a logical consequence of this SLR I exclude book reviews and literature reviews.

\section{Methodology}

I do not include studies which do not report on methods applied (unclear methodology) for data collection and analysis. I have subjectively, and not objectively, taken the criteria and limitations presented by me as a researcher. Nonetheless, I try to make decisions based on evidence. For example, I have established the timeframe based on the German Occupational Safety and Health 
employment protection law. My thesis is still a qualitative research project and cannot consistently maintain objectivity.

The following table summarised the inclusion and exclusion criteria of my study (see also chapter 2.1):

Table 13: Inclusion/exclusion criteria.

\begin{tabular}{|c|c|c|}
\hline Parameters & Inclusion criteria & Exclusion criteria \\
\hline Locations & Europe, Australia and USA & Non-Europe, non-Australia and non-USA \\
\hline Language & $\begin{array}{l}\text { Literature written in English and } \\
\text { German }\end{array}$ & $\begin{array}{l}\text { Literature NOT written in English and } \\
\text { German }\end{array}$ \\
\hline Time frame & Literature published after 1996 & Literature published before 1996 \\
\hline Subjects & $\begin{array}{l}\text { Workplace health and well-being } \\
\text { promotion }\end{array}$ & $\begin{array}{l}\text { Workplace health promotion (WHP) } \\
\text { focusing only on physical side }\end{array}$ \\
\hline Relevance & Salutogenesis & Pathogenic or deficit perspective \\
\hline $\begin{array}{l}\text { Context and } \\
\text { population }\end{array}$ & SMEs in Germany and their employees & $\begin{array}{l}\text { Non SMEs, non SMEs Germany, non } \\
\text { employees }\end{array}$ \\
\hline Outcome & $\begin{array}{l}\text { Studies which do report on health- } \\
\text { oriented interventions }\end{array}$ & $\begin{array}{l}\text { Studies which do not report on health- } \\
\text { oriented interventions }\end{array}$ \\
\hline Study type & Primary research & $\begin{array}{l}\text { Book reviews, literature reviews, opinion } \\
\text { pieces, policy documents }\end{array}$ \\
\hline Methodology & $\begin{array}{l}\text { Studies containing findings which are } \\
\text { based on quantitative and/or } \\
\text { qualitative methods (data collection } \\
\text { and analysis) }\end{array}$ & $\begin{array}{l}\text { Studies which do not report on methods } \\
\text { applied (unclear methodology) for data } \\
\text { collection and analysis }\end{array}$ \\
\hline
\end{tabular}

The criteria of including and excluding parameters leads to problems due to their application to quantitative and qualitative research in relation to identifying the year of data collection (parameter: time frame) and the applied methods which are often not exactly described (parameter: study type). The problem of locating qualitative research, and then determining the relevance of studies to be included within reviews, has been highlighted by the work on the systematic review of qualitative research (Campbell et al., 2003). I have based finding strategies for conventional (quantitative) systematic reviews are based on achieving statistical 
representativeness which is not a feature of qualitative research. Nevertheless, I chose this systematic way of reviewing because I want to make sure that I do not miss important topics and studies. The application of the above explained parameters results in 40 studies which fit the inclusion criteria for this review.

Table 14: Sources of the studies meeting the inclusion criteria.

\begin{tabular}{|c|c|}
\hline Sources & $\begin{array}{l}\text { Numbers of studies meeting } \\
\text { the inclusion criteria }\end{array}$ \\
\hline PubMed & 0 \\
\hline $\begin{array}{l}\text { Business Source Complete, PyscARTICLES, Psychology and Behavioural } \\
\text { Sciences Collection, PsycINFO, SocINDEX, CINAHL } \\
\text { (all via EBSCO) }\end{array}$ & 23 \\
\hline $\begin{array}{l}\text { WorldCat.org, Emerald Group Publishing Limited, SpringerLink, Wiley } \\
\text { Online Library, Nexis Uni, ProceedingFirst, WorldCat, ArticelFirst, } \\
\text { Electronic Collections Online, ABI/INFORM Global, Electronic Books, } \\
\text { OAlster, Medline, SPORTDiscus, Science Direct, SAGE Journals (all via } \\
\text { wordcat and Discover Library Resources of University of } \\
\text { Gloucestershire) }\end{array}$ & 11 \\
\hline Open Access to Theses and Dissertations & 0 \\
\hline Google Books/Google Scholar & 0 \\
\hline Websites (institutions, foundations, organizations) & 6 \\
\hline Total & 40 \\
\hline
\end{tabular}

\subsection{Quality assessment}

The aim of the qualitative assessment of qualitative studies is to exclude studies that do not provide reliable answers to the review question of the literature review. I check the quality of the studies according to a specific principle recommended by the EPPI-Centre (The Evidence for Policy and Practice Information and Co-ordinating Centre). Dixon-Woods et al. (2001) emphasize the need for a structured approach to quality assessment for qualitative studies. They 
also recognize the difficulties of achieving consent on the criteria that might establish quality standards. Tranfield (2003) adds that in the management field it may be possible to implement a quality assessment stage, but management researchers usually rely on the implicit quality rating of a particular journal rather than formally applying any quality assessment method. Until the debate on quality appraisal moves closer to resolution, this is likely to be an area of continuing contention and therefore of differing practice (Jensen et al., 1996; Sandelowski et al., 1997).

With the different methods and their application in different sciences criticism arises that SLR in social science does not often meet the quality criteria (validity, reliability) such as SLR in medicine or health sciences. In situations where a full systematic review is not feasible for example for doctoral students the proposed approach to more systematic verification of evidence can significantly improve the reliability of the verification results (Haddaway et al., 2015). Reviewing evidence in a more systematic way can substantially improve the reliability of my review findings. Besides, the literature review profits from the quality assessment criteria developed by the EPPI-Centre (The Evidence for Policy and Practice Information and Co-ordinating Centre) (Oliver et al., 2005) as mentioned before and which are listed as questions (see table 15). 
Table 15: Quality assessment criteria, based on EPPI-Centre criteria (Shepherd et al., 2006).

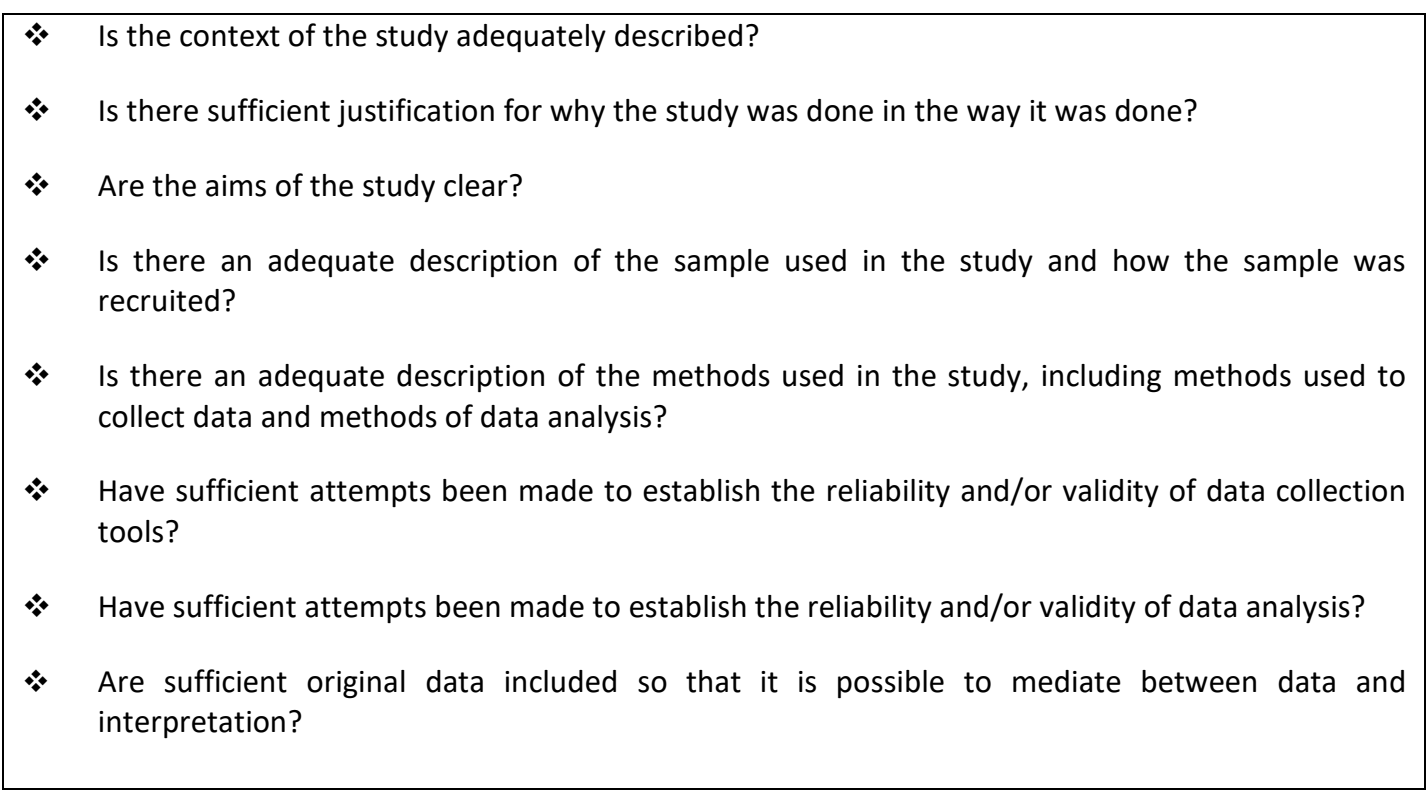

In the following figure 14 I develop an appraisal system in order to make transparent the application of the quality criteria, and to make transparent the foci of my study. I base the foci on the review question of the literature review and they represent the context of my thesis (WHP etc.). In this step I systematically check the quality of the 40 studies and check if they deliver results that are relevant to my study. For that I create an appraisal system that clearly illustrates the relevant criteria (marked with A, B, C, D and E) of the 40 studies (see figure 14). The quality assessment criteria are marked with ' $A$ ' in the appraisal system. The foci 'health and well-being of employees' marked with ' $\mathrm{B}$ ', the foci 'workplace health promotion' get ' $C$ ', the foci 'salutogenesis (SoC)' get ' $D$ ', the foci 'enterprises' get ' $E$ '. Since the scoping study has shown that there is a research gap of salutogenic WHP in the context of SMEs, I focus on enterprises of different sizes in the appraisal system. I choose studies that are embedded in businesses context. The following figure summarises the relevant criteria of the appraisal system: 
Figure 14: Appraisal system of the quality assessment criteria and the topic foci.

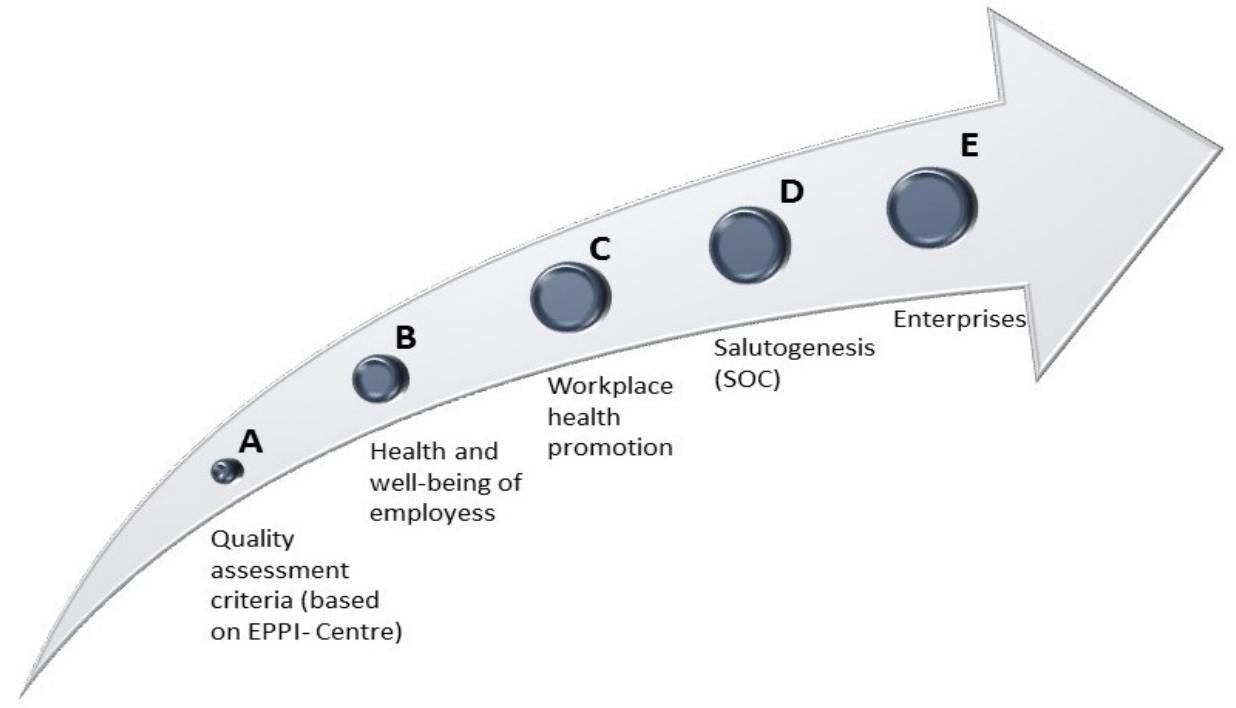

The 40 studies are again full text read and I check which of the criteria (A, B, C, D and $E$ ) of the appraisal system they fulfil. If a study meets all the criteria, it means that it can provide important answers to my review question. I only use studies with grade $A, B, C, D$ and $E$ in the final synthesis (see table 16). The table in appendix 9.3 shows the grading status of all 40 studies and gives information if, or to what extent, the studies fulfil the criteria of the appraisal system.

Table 16: Studies which meet the quality assessment criteria.

\begin{tabular}{|c|c|c|c|c|c|c|}
\hline No. & Authors & Place, time & Title & Rate & $\begin{array}{l}\text { Samples' } \\
\text { environment }\end{array}$ & Methodology \\
\hline 1 & $\begin{array}{l}\text { Nilsson, P. } \\
\text { Andersson, I.H. } \\
\text { Ejlertsson, G. } \\
\text { Troein, M. }\end{array}$ & $\begin{array}{l}\text { 2012, } \\
\text { Sweden }\end{array}$ & $\begin{array}{l}\text { Workplace health } \\
\text { resources based on sense } \\
\text { of coherence theory }\end{array}$ & ABCDE & University & $\begin{array}{l}\text { Salutogenic } \\
\text { approach, } \\
\text { focus group } \\
\text { interviews, } \\
\text { deductive analysis }\end{array}$ \\
\hline 2 & $\begin{array}{l}\text { Bauer, G.F. } \\
\text { Jenny, G. F. }\end{array}$ & $\begin{array}{l}2007, \\
\text { Switzerland }\end{array}$ & $\begin{array}{l}\text { Development, } \\
\text { Implementation and } \\
\text { Dissemination of } \\
\text { Occupational Health } \\
\text { Management (OHM): } \\
\text { Putting salutogenesis into } \\
\text { practice }\end{array}$ & ABCDE & University & $\begin{array}{l}\text { preliminary } \\
\text { dissemination } \\
\text { study, the } \\
\text { longitudinal } \\
\text { reflection-group } \\
\text { design, quantitative } \\
\text { approach, } \\
\text { questionnaire }\end{array}$ \\
\hline
\end{tabular}




\begin{tabular}{|c|c|c|c|c|c|c|}
\hline 3 & $\begin{array}{l}\text { Feldt, T. } \\
\text { Kinnunen, U. } \\
\text { Mauno, S. }\end{array}$ & $\begin{array}{l}\text { Finland, } \\
2000\end{array}$ & $\begin{array}{l}\text { A mediational model of } \\
\text { sense of coherence in the } \\
\text { work context: a one-year } \\
\text { follow-up study }\end{array}$ & ABCDE & University & $\begin{array}{l}\text { One-year follow-up } \\
\text { study, quantitative } \\
\text { approach, } \\
\text { questionnaires, } \\
\text { statistical analysis }\end{array}$ \\
\hline 4 & $\begin{array}{l}\text { Bringsén, A. } \\
\text { Andersson, H. I. } \\
\text { Ejlertsson, G. } \\
\text { Troein, M. }\end{array}$ & $\begin{array}{l}\text { Sweden, } \\
2012\end{array}$ & $\begin{array}{l}\text { Exploring workplace } \\
\text { related health resources } \\
\text { from a salutogenic } \\
\text { perspective: Results from } \\
\text { a focus group study } \\
\text { among healthcare } \\
\text { workers in Sweden }\end{array}$ & ABCDE & University & $\begin{array}{l}\text { Qualitative } \\
\text { approach, focus } \\
\text { group interviews, } \\
\text { qualitative content } \\
\text { analysis }\end{array}$ \\
\hline 5 & $\begin{array}{l}\text { Vogt, K. } \\
\text { Hakanen, J. J. } \\
\text { Jenny, G. J. } \\
\text { Bauer, G.F. }\end{array}$ & $\begin{array}{l}\text { Finland } \\
\text { Switzerland, } \\
2016\end{array}$ & $\begin{array}{l}\text { Sense of Coherence and } \\
\text { the Motivational Process } \\
\text { of the Job-Demands- } \\
\text { Resources Model }\end{array}$ & $A B C D E$ & University & $\begin{array}{l}\text { Longitudinal study, } \\
\text { quantitative } \\
\text { approach, } \\
\text { questionnaire, } \\
\text { statistical analysis }\end{array}$ \\
\hline 6 & Nilsson, P. & $\begin{array}{l}\text { Sweden, } \\
2010\end{array}$ & $\begin{array}{l}\text { Enhance your workplace!: } \\
\text { A dialogue tool for } \\
\text { workplace health } \\
\text { promotion with } \\
\text { salutogenic approach }\end{array}$ & ABCDE & University & $\begin{array}{l}\text { Quantitative and } \\
\text { qualitative } \\
\text { approach, } \\
\text { questionnaires }\end{array}$ \\
\hline 7 & $\begin{array}{l}\text { Vinje, H. F., } \\
\text { Ausland, L.H. }\end{array}$ & $\begin{array}{l}\text { Sweden, } \\
2013\end{array}$ & $\begin{array}{l}\text { Salutogenic presence } \\
\text { supports a health } \\
\text { promoting } \\
\text { work life }\end{array}$ & ABCDE & University & $\begin{array}{l}\text { Qualitative } \\
\text { approach, } \\
\text { explorative } \\
\text { design, interviews, } \\
\text { thematic analysis }\end{array}$ \\
\hline 8 & $\begin{array}{l}\text { Saksvik, P. } \varnothing . \\
\text { Olaniyan, O. S. } \\
\text { Lysklett, K. } \\
\text { Lien, M. } \\
\text { Bjerke, L. }\end{array}$ & $\begin{array}{l}\text { Norway, } \\
2015\end{array}$ & $\begin{array}{l}\text { A process evaluation of a } \\
\text { salutogenic intervention }\end{array}$ & ABCDE & University & $\begin{array}{l}\text { Quantitative and } \\
\text { qualitative } \\
\text { approach, } \\
\text { questionnaires, } \\
\text { interviews, } \\
\text { thematic analysis }\end{array}$ \\
\hline 9 & $\begin{array}{l}\text { Vogt, K. } \\
\text { Jenny, G. J. } \\
\text { Bauer, G. F. }\end{array}$ & $\begin{array}{l}\text { Switzerland, } \\
2013\end{array}$ & $\begin{array}{l}\text { Comprehensibility, } \\
\text { manageability and } \\
\text { meaningfulness at work: } \\
\text { Construct validity of a } \\
\text { scale measuring work- } \\
\text { related sense of } \\
\text { coherence }\end{array}$ & ABCDE & University & $\begin{array}{l}\text { Quantitative } \\
\text { approach, } \\
\text { questionnaires, } \\
\text { statistical analysis }\end{array}$ \\
\hline
\end{tabular}




\begin{tabular}{|c|c|c|c|c|c|c|}
\hline 10 & $\begin{array}{l}\text { Ejlertsson, G. } \\
\text { Heijbel, B. } \\
\text { Andersson, H. I. }\end{array}$ & $\begin{array}{l}\text { Sweden, } \\
2018\end{array}$ & $\begin{array}{l}\text { Recovery, work-life } \\
\text { balance and work } \\
\text { experiences important to } \\
\text { self-rated health: A } \\
\text { questionnaire study on } \\
\text { salutogenic work factors } \\
\text { among Swedish primary } \\
\text { health care employees }\end{array}$ & ABCDE & University & $\begin{array}{l}\text { Qualitative } \\
\text { approach, } \\
\text { questionnaires }\end{array}$ \\
\hline
\end{tabular}

After application of the appraisal system, 10 studies remained which meet all the criteria ( $A$ to $E$ ) of the appraisal system (see grading system of all studies in the appendix 9.3). The final ten studies, which are selected with the help of the appraisal system, should provide the highest possible response to the review question and, of course, ensure the quality of the final studies. It has to be noticed that I define the criteria for the study search, and therefore the selection of studies is not objective. I try to introduce as much objectivity as possible through the transparency of the review process and the strict method (PICOC, review question, inclusion criteria etc.) used for the SLR.

By excluding the studies which do not have the desired grades from $A$ to $E$, the quality assessment enabled a high degree of reliability and validity by the findings of the studies to be synthesised. In other words, the outsourcing of thirty studies with the help of the appraisal system still enabled the synthesis to continue with a broad range and depth of findings on health and well-being promotion of employees in terms of salutogenesis. At this point the studies included for the final synthesis covered a range of different subject fields such as salutogenic work factors, workplace health resources, WHP tool with a salutogenic approach, measurement of Work-SoC, usability of SoC in the workplace, evaluation of salutogenic interventions etc.

Four of the final studies have a qualitative approach. Four have a quantitative approach, and two studies use a quantitative and qualitative approach (see chapter 2 , table 16$)$. The final studies with a quantitative approach try to test the SoC in the working context, the qualitative studies involved aims to explore 
experiences or meanings of workforces regarding WHP in terms of salutogenesis. The next chapter 3.6 takes a closer look at the final studies by looking more closely at their concepts or themes and their results to then work out relationships of studies in the next step.

\subsection{Integrating and synthesising qualitative research}

\subsubsection{Rationale of choosing synthesis method}

The review question serves as a focus in the phase of literature research as mentioned before and sets also a framework for choosing an analysis method to synthesise the study findings. With the question of this review 'Which salutogenic factors support the promotion of health and well-being of the workforce in SMEs?' I want to address factors that might influence successful implementation of health and well-being promotion into SMEs. Ridely (2012) suggests a narrative synthesis instead of meta-analysis when systematic reviews are not about effectiveness of interventions. In addition, final studies of this review report also on qualitative research, therefore narrative synthesis involves using text and words to present findings of the study being reported (Popay et al., 2006). Narrative synthesis is also helpful when studies being reviewed are very diverse in nature as are the final studies of my review (Ridley, 2012). Nevertheless, the challenge for this review is to hold the balance between developing practical methods for synthesising the findings and to preserve the integrity of individual studies. Narrative synthesis, as an appropriate method, is drawn upon to synthesise qualitative findings on health and well-being promotion factors of workforces.

\subsubsection{Method of narrative synthesis}

„Unlike meta-analysis, narrative synthesis does not rest on an authoritative body of knowledge or on reliable and rigorous techniques developed and tested over time" (Popay et al., 2006, p. 6). Because of the absence of such body of 
knowledge, the Cochrane handbook recommends the need for the methodological foundation of narrative synthesis, if systematic reviews produced to inform the choice and implementation of interventions are to be credible (Higgins et al., 2011). For this reason the narrative synthesis of this review follows the 'guidance on the conduct of narrative synthesis in systematic reviews' by Popay et al. (2006), the guideline of Ridley (2012) and the EPPI Centre. By using the guidance of Popay et al. (2006) my review focuses on the synthesis of evidence on factors influencing the implementation of interventions rather than effectiveness because it follows the rationale of my study (see chapter 3.6.1). I would like to make sure that the synthesis of my review follows proven and accepted recommendations like the guideline of the EPPI-Centre. Hence, the synthesis of this review follows four steps:

1) Developing a theory of how the intervention works, why and for whom This can be derived from the tool 'vote counting', "[...] which essentially tallies the number of each type of result in relation to an intervention or implementation factor, giving different weightings where appropriate" (Ridley, 2012, p.196). In other words, it is about simply comparing the number of positive studies (studies showing benefit of WHP in terms of salutogenesis) with the number of negative studies (studies showing harm regarding health promotion in terms of salutogenesis). Therefore, I use a table to display the results.

2) Developing a preliminary synthesis

This can be derived from a textual summary of the ten studies. Therefore, I use again the table and add information about participants, interventions and significant factors of each final study to it.

3) Exploring relationships within and between studies

This can be derived from the techniques of 'cross-case thematic analysis' for drawing out the main themes of the studies and technique "'Lines-of-argument synthesis'.

4) Assessing the robustness of the synthesis

This can be derived for my review based on three criteria: methodological soundness, appropriateness of study design, and the relevance of the study review question. Therefore, I make a composite assessment of the overall weight that a study carries (Ridley, 2012). This aims in a well-described grading system 
which gives information about how the weightings are applied to the overall results of the synthesis.

The next chapter 3.6.3 describes each step of narrative synthesis of my review in detail and presents also an overview of the results.

\subsubsection{Synthesising qualitative research on health and well-being promotion} of workforces in terms of salutogenesis

With the first step of synthesis I aim to develop a theory of how the health and well-being intervention works, why and for whom. I compare the ten final studies and examine which study lead to positive and constructive outcomes regarding application of salutogenesis in WHP of workforces or which studies do not show positive effects regarding salutogenesis in WHP. Each study then casts a 'vote' in support of the relationship of health and well-being promotion of workforces and the theory of salutogenesis. 'Vote-counting' is the method which is recommended when the studies have used incompatible measures or the information reported from the studies is very limited (Mohagheghi et al., 2004).

To review the relationship between WHP and salutogenesis, I re-read the ten studies in this regard. By reading and checking the ten studies if they present findings which show benefit of WHP in terms of salutogenesis it is obvious that there is positive resonance to use salutogenesis in WHP, to positive work experience and to resource-orientation of WHP (see table in the appendix 9.4). Of course, the ten studies used salutogenesis differently (some as the basis of a questionnaire or as the basis of a tool) and explore employee health in different contexts (hospital, care settings, consulting agencies etc.) but all ten studies rated the use of salutogenesis in WHP, positive work experiences and the resource orientation as constructive and positive regarding employee health and employee well-being. The ten studies regarding salutogenesis will be discussed later in this chapter. The preliminary theory after the first step of the synthesis can be formulated: The consideration of salutogenesis, positive working experiences and 
the resource view in WHP shows positive effects on the health and well-being of employees.

With the second step of synthesis I want to add information about participants, interventions and significant factors of each final study. In order to get information of, for example, significant factors, I make a textual summary of the final studies. The aim of this synthesis step is to develop a preliminary synthesis before I explore relationships within and between studies. The results of the second step are displayed in the table in the appendix 9.5 .

As Ridley (2012) recommends, a consistent format like the table in the appendix (9.5) helps me to compare useful information of the synthesis of final studies. Since the studies give very little information about participating companies, company size etc., it is difficult to make a comparison. As already mentioned, the research gap in salutogenic health and well-being promotion in SMEs is to be closed with my study. For the preliminary synthesis it means that I focus on:

1. What are the interventions of WHP in terms of salutogenesis and

2. What are the significant factors related to implementation of WHP in terms of salutogenesis?

The first comparison of the interventions of the final studies shows that they use the theory of salutogenesis to develop, to test or evaluate a health and well-being programme, model or questionnaire regarding workforce health and well-being for example a systemic-salutogenic model of Occupational Health Management (OHM) by Bauer et al. (2007). Bauer et al. (2007) create a survey on the use of OHM interventions based on SoC by questionnaire. The study of Nilsson et al. (2012) has a salutogenic approach to WHP because they conducted a deductive analysis made with the SoC theory as a framework. Work-related specific enhancing resources (SER) were identified and analysed into the three components of SoC: comprehensibility, manageability, and meaningfulness. Feldt 
et al. (2000) test a mediational model appropriate for explaining the effects of psychosocial work characteristics on general well-being, and on occupational wellbeing via SoC in a one-year follow-up study. The methodology of the study by Bringsén (2012) shows that SoC is used to analyse the study findings in order to interpret the needs of workers. Another study of this review used SoC to combine it with another model called 'job-demands-resources model' by Vogt et al. (2016). It examines the various roles played by $\mathrm{SoC}$ in the motivational process described by the job-demands-resources model (JD-R model). The study brings together two fields: Antonovsky's salutogenic approach, and the JD-R model, which has been highly influential in occupational health psychology research. Vinje et al. (2013) investigate with their study in salutogenic factors with the intention to keep their employees present at work. They explore the qualities of presence and the dynamics of health in the workplace, both individually and in groups. Vogt et al. (2016) modify the SoC for the workplace context (Work-SoC) which might be used as a screening instrument to detect the need for further investigation and to derive interventions that enhance the health-promoting quality of working environments. Ejlertsson et al. (2016) create a questionnaire, which has a salutogenic perspective and combine it with other instruments (Salutogenic Health Indicator Scale and Work Experience Measurement Scale). The variety of interventions by the $\mathrm{SoC}$ of final studies shows that $\mathrm{SoC}$ is flexible for implementation in WHP and can be used both as a basis for analysis and as an instrument for data gathering and can be combined with other models.

The significant factors of the final studies (see table in appendix 9.5) for example 'providing a resourceful working environment' show that it is important to evaluate the needs of employees regularly and to create a work environment that is imaginative. 'Providing a resourceful working environment' can help to build employee SoC and the creation of additional job resources, leading to positive outcomes such as work engagement (Vogt et. al., 2013). The interventions of the final studies demonstrate that they explore and test the SoC (in addition to the 
goal of promoting health) also regarding the motivation and commitment of employees (e.g. Vinje et al. 2013, Vogt et al., 2016). The study by Vinje et al. (2013) is the only study to investigate salutogenic factors regarding senior leaders and co-workers, which links up to my study, as I also include top managers and employees in my study. The other final studies focus only on employees with their interventions. As decision makers on WHP interventions are top managers, it is essential to include them in all WHP steps and to include their opinions, wishes and concerns, as my study does.

The importance of a good work climate and the importance of work conditions in order to make good psychosocial experiences were noticed (Vogt, et. al.2016; Nilsson et. al., 2010). A further significant factor of the final studies demonstrates the importance of a resource-oriented view on WHP (Vogt et. al., 2016). The theme 'recovering at work' also seems to be an important criterion when it comes to WHP in terms of salutogenesis (see all significant factors of the final studies in the appendix 9.5). It can be deduced at this synthesis step that the current research on the promotion of health and well-being of employees in the workplace uses salutogenesis in order to promote workforces in a strengthoriented and resource-oriented way and leaves the ones of the pathogenic way behind. Current research is no longer focusing on the elimination of disease symptoms but on strengthening mental and physical health of workforces. SoC seems to be a very well-accepted theory in health promotion, and can be incorporated differently into WHP processes, as the final studies of my review show. With the theory of salutogenesis as its foundation, WHP interventions have been given a new focus which could be seen as investing in the needs of employees'. Salutogenesis has not really taken place yet in businesses (outside the health care sector) for the promotion of employees' health and well-being, as the final study settings show. 
With the next step of synthesis, I want to explore the relationships within and between studies. This step can be derived from different techniques that help me to synthesise the final studies. The synthesis makes use of these three techniques:

1. Reciprocal translation analysis - this entails examining the key concepts across each study. An attempt is made to translate the concepts into each other. Judgements about the ability of the concept of one study to capture concepts of others are based on the attributes of themes themselves, including cogency, economy and scope. The concept which is 'most adequate' is chosen;

2. Refutational synthesis. The key concepts and themes in each study are identified and contradictions between the reports are characterised. The 'refutations' are examined, and an attempt made to explain them;

3. Lines of argument synthesis - involves building a general interpretation grounded in the findings of the separate studies (similar to comparative analysis of Grounded Theory).

The findings of the final studies may be about the same subject, but they are at different conceptual levels as the second step of the synthesis showed (see table in the appendix 9.5). Because of this reason, the reciprocal translation synthesis and refutational synthesis are not attempted. As compensation, the cross-case analysis from Miles and Huberman (1994) is utilised to address the problem of synthesizing the variety of the research findings. Also Grounded Theory's comparative techniques are applied (Glaser et al., 1998). 'Lines-of-argument synthesis', as the third point of the Meta-ethnographic method, is utilised.

For synthesizing research on health and well-being promoting factors of workforces the remaining ten studies cover a broad range of topic areas as mentioned before. Because of the trouble with a broad range of subject areas, the synthesis is conducted in two parts:

1. Cross-case thematic analysis for drawing out the main themes of the studies.

2. Lines-of-argument synthesis (Noblit et al., 1988), where findings and concepts of the individual studies are compared to generate a new interpretative context.

The following sections display these two techniques in more detail. 


\section{Cross-case thematic analysis}

Miles and Huberman (1994) argue that the method aims to 'deepen understanding and explanation' and refer therewith to the Meta-ethnography viewpoint of Noblit and Hare (1988) and also Glaser and Strauss' Grounded Theory (1998). The technique to synthesise by Miles and Huberman (1994) is to array matrices, tables and models to aid cross-case analysis that are not used specifically in this synthesis. The principles behind cross-case analysis do confirm the techniques utilized (McDermott et al., 2004). In particular, Miles and Huberman (1991) emphasise a thorough understanding of each case to avoid superficiality; to avoid aggregation, that is, cases should not be 'idly lumped' on the basis of similarities and differences; the preservation of case specifics during analysis; inquiry into deviant cases; and importantly, to avoid forcing the analysis too quickly. Researchers must recognise that inductive grounding takes time.

For the review a cross-case thematic analysis is accomplished where each study is treated as a single case with multiple findings. Studies with all grades $(A, B, C, D$ and $\mathrm{E}$ ) in the quality appraisal are read repeatedly. The aim of this assessment is to sensitise the main findings of the research (McDermott et al., 2004). The following themes are identified from interpretations, explanations and descriptions of the ten studies:

- Recovery in the workplace

- Sense of security or stability in the work role

- Knowledge about implementation and evaluation of WHP interventions

- A good organisational climate at work

- Providing a resourceful working environment or a resource-oriented view on workplace health promotion

- Usefulness of the SoC theory in workplace health promotion (WHP)

- Strengthen employees' competency (competency consists of the elements introspection, sensibility, reflection, and action/active mastery of the situation)

- Learning of employers and employees to explore their own health

- Leadership (e.g. leadership action and attitude, employees' involvement, made sure that the employees enjoy their work in a pleasant atmosphere) 
- Communication (e.g. come together outside of daily work to learn how to communicate better with colleagues, and to get to know each other better)

- Resistance of employees to change

- Involvement of employees

- Relationship between work-life balance and health

- Positive work experiences (to be able to come to work with joy and to experience work as meaningful and challenging)

After identification of the main themes of the final studies, I make notes about general categories under which these findings would be suitably grouped and coded. I decide to check the final studies regarding practicability of findings. This categorisation helps me (in the last synthesis step) to set the review findings in relation to my review question. Therefore, I use the fourteen themes in the next step as codes and I also categorise them regarding practicality for each study. I read each of the ten studies again closely and I code and categorise the research interpretations, explanations and descriptions using the fourteen themes. For a better clarification for each study I develop a table which has the findings from the study aggregated under each code (McDermott et al., 2004), and also gives information about practicality of study findings. The following table shows an example:

Table 17: Example of table of the study of Ejlertsson et al. (2018).

\begin{tabular}{|l|l|l|l|}
\hline Codes & Themes & $\begin{array}{l}\text { Practical findings about } \\
\text { initiatives which promote the } \\
\text { health and well-being of } \\
\text { employees }\end{array}$ & study \\
\hline 1 & Recovery in the workplace & Yes & Yes \\
\hline 2 & Sense of security or stability in the work role & No \\
\hline 3 & $\begin{array}{l}\text { Knowledge about implementation and evaluation of WHP } \\
\text { interventions }\end{array}$ & No \\
\hline 4 & A good organizational climate at work & Yes & No \\
\hline 5 & $\begin{array}{l}\text { Providing a resourceful working environment or a resource- } \\
\text { oriented view on workplace health promotion }\end{array}$ & Yes & Yes \\
\hline 7 & $\begin{array}{l}\text { Usefulness of the SoC theory in workplace health } \\
\text { promotion (WHP) }\end{array}$ & Strengthen employees' competency (competency consists & Yes \\
\hline
\end{tabular}




\begin{tabular}{|l|l|c|c|}
\hline & $\begin{array}{l}\text { of the elements introspection, sensibility, reflection, and } \\
\text { action/active mastery of the situation) }\end{array}$ & & \\
\hline 8 & $\begin{array}{l}\text { Learning of employers and employees to explore their own } \\
\text { health }\end{array}$ & Yes & No \\
\hline 9 & $\begin{array}{l}\text { Leadership (e.g. leadership action and attitude, employees' } \\
\text { involvement etc.) }\end{array}$ & & Yes \\
\hline 10 & $\begin{array}{l}\text { Communication (e.g. come together outside of daily work } \\
\text { to learn how to communicate better with colleagues, and } \\
\text { to get to know each other better) }\end{array}$ & & No \\
\hline 11 & Resistance of employees to change & - & Yes \\
\hline 12 & Involvement of employees & Yes & Yes \\
\hline 13 & Relationship between work-life balance and health & Yes & Yes \\
\hline 14 & $\begin{array}{l}\text { Positive work experiences (to be able to come to work with } \\
\text { joy and to experience work as meaningful and challenging) }\end{array}$ & & Yes \\
\hline
\end{tabular}

While moving between the ten studies and the tables with coded findings, I compare the interpretations, descriptions and findings of the research. By analysing the differences and commonalities an emphasis on preserving the context of individual interpretations and explanations take place (McDermott et al., 2004). I even record discussions with experts of the University of Applied Sciences (Fachhochschule des Mittelstands (FHM)) to debate and evaluate the fourteen themes. Result of the interim synthesis are the following themes which are emerging as important interpretive context for understanding health and wellbeing promoting factors of workforces in terms of salutogenesis:

- $\quad$ Recovery in the workplace

- $\quad$ Providing a resourceful working environment

- Positive work experiences (to be able to come to work with joy and to experience work as meaningful and challenging)

- Strengthen employees' competency (introspection, sensibility, reflection, and action/active mastery of the situation)

- Usefulness of the SoC theory in workplace health promotion

- Leadership (e.g. leadership action and attitude, employees' involvement, made sure that the employees enjoy their work in a pleasant atmosphere) 


\section{'Lines-of-argument synthesis'}

The technique 'lines-of-argument synthesis' was utilised by Noblit and Hare (1988) and is a means to compare findings or concepts of the individual studies to generate a new interpretative context. It is analogous to Grounded Theory's general method of comparative analysis method by Corbin and Strauss (2014). The comparative analysis used for the 'lines-of-argument synthesis' compare the ten primary studies and the tables with codes and categorised developed in the phase of the synthesis. For promoting the comparative analysis for this synthesis, I develop a table for each of the identified codes (outlined in the previous section). The code table displays the main findings of the studies under each code. With the help of the table I can compare more easily the concepts, interpretations and findings for a particular code for example 'Usefulness of the SoC theory in workplace health promotion' across the ten studies. The analysis can move away from the particular to the general (McDermott et al., 2004) and opens the area of abstract thinking and creativity.

Using comparisons, the features of the data consider the relationship between the studies' concepts to produce new interpretative possibilities and applied explicit qualitative data-analysis techniques of Grounded Theory (Corbin et al., 2014). Strauss and Corbin (2014) explain that theoretical comparisons are tools (a list of properties) for looking at something somewhat objectively, rather than naming or classifying without a thorough examination of the object at the property and dimensional levels. Another qualitative data analysis technique, using memos and visual devices, is derived from Grounded Theory. As McDermott et al. (2004) note both techniques are analytical and conceptual, rather than descriptive. This technique is helpful because I am able to gain analytical distance (Corbin et al., 2014). Helpful also is to utilise memos and visual devices because it makes possible to move from the findings to concepts and statements. "Analysis across studies in one sense is partially bringing together [...] findings, which in the light of the whole, have greater significance." (McDermott et al., 2004, p. 25). As Noblit 
and Hare (1988) argue, the aim of the lines-of-argument synthesis is to discover a whole among a set of studies.

After this step, it can be noticed that promoting health and the well-being of employees requires a concept that is geared towards the resources and needs of employees. The workplace has meanwhile become an important part of health promotion and has a great influence on the health and well-being of humans. The workplace should be designed in such a way that employees can make good experiences and their skills are strengthened. The resource and needs oriented approach in WHP should be lived by top management and their leaders.

The last step of this synthesis is to 'assessing the robustness of the synthesis' as Ridley (2012) recommends. As mentioned before, this can be derived based on three criteria: methodological soundness, appropriateness of study design checked with relevance to the research objectives (factors of the study by Bruch and open questions (see table 2)), and the relevance of the study review question or study research questions. Therefore, I make a composite assessment of the overall weight that a study carries (Ridley, 2012). The aim is a well-described grading system which gives information about how the weightings are applied to the overall results of the synthesis. I examine the final studies for relevance regarding review question, relevance regarding study research questions, for validity shown by methodological soundness and for appropriateness of study design (relevance regarding research objectives) (see table in appendix 9.7). If a study fulfils a condition (see the conditions displayed in four columns of the table in appendix 9.7) it is provided with a checkmark in the table. This step demonstrates that the final studies give important and relevant information regarding the review question and the study research questions. Moreover, they show validity by their methodology checked by the appraisal system of the quality assessment criteria (see chapter 3.5). Eight of ten studies are related to the research objectives of this study and show similarities with the nine factors of the 
study by Bruch et al. (2013) (see table 1) in their themes (e.g. 'challenging and supporting' has a connection to the review finding 'strengthen employees' competency').

In chapter 5 'Discussion of study findings in the context of review findings' I compare the review findings with my study findings and I also make references to the nine factors of the study by Bruch et al. (2013), and the theory of salutogenesis. After the last synthesis step of assessing the robustness of synthesis, I represent the common concept of final studies in relation to my study concept in the next chapter.

\subsection{Discussion of final studies}

This section presents the common concepts of final studies that have been found through the four synthesising steps of the study. Even if the studies in this thesis are not investigating the complex concept of health and well-being promotion in working context, but with WHP interventions based on salutogenesis, this study can provide important impulses for the health and well-being of employees. Previous research of health and well-being promotion in the working context shows the benefit and usefulness of the theory of salutogenesis based in WHP (Nilsson et al., 2010; Feldt et al., 2000, Ejlertsson et al. (2016). Nevertheless, SMEs are not given enough attention as a field of research in salutogenic health promotion, as the final studies show. The research settings of the final studies are mostly conducted in organisations in the health care sector. It seems that the commitment and understanding of health promotion is higher among employees in the health sector than, for example, in manufacturing companies.

Further, the synthesis shows that there is common concept in using the theory of salutogenesis as a new way of thinking in WHP, away from pathogenesis view to positive or resource orientated promotion of employees' health and well-being (Saksvik et al., 2015; Vinje et al., 2013). In the sense of Positive Psychology, the 
studies provide results which give answers to the question 'what keeps employees healthy' and not 'what makes them sick'. Moreover, the final studies with their interventions establish the importance of promotion mental health or well-being of employees (Nilsson et al., 2010; Bauer et al., 2007), and it was also checked that the SoC of employees is related to well-being (Vogt et al. 2016, Vinje et al., 2013). The synthesis checked that there is a common concept in using salutogenesis as a theory particularly to promote the mental health of employees. This fits with my study concept to find WHP interventions based on SoC to promote the well-being (in addition to health) of employees. The intention to grasp or fulfil the physical and mental needs of employees in terms of the theory of salutogenesis is a common concept in all studies. This is evidenced by the theme 'providing a resourceful working environment for employees', which appears in various final studies (Nilsson et al., 2010, Vinje et al., 2013). In addition to the theme 'providing a resourceful working environment', the other themes of the interim synthesis follow the same intention to meet the employees' needs (e.g. 'positive work experiences'). Even though the studies apply the theory of salutogenesis differently (e.g. in qualitative and/or quantitative approach, in data collection and/or data analysis), but it can be summarised as a helpful theory in WHP that focuses on humans, takes their needs into account and therefore shows positive effects in WHP. This review shows the trend in WHP towards more humanity with the implementation of the theory of salutogenesis and demonstrates it has not arrived in SMEs yet. Further discussions of the review findings and study findings in respect of salutogenic WHP in SMEs take place in the last chapter 'Conclusions of salutogenic WHP research in SMEs'.

The following chapter displays the review findings in detail to answer the three study research questions. 


\subsection{Review findings for answering the research questions}

This review evaluated the literature since 1996 and is based on a concrete review question. The synthesis follows the narrative way and is of quantitative and qualitative studies using the cross-thematic analyses and lines-of-argument synthesis' described in chapter 3.6.2. It indicates health and well-being promoting factors in terms of salutogenesis that can be implemented by businesses in the form of providing a resourceful working environment, strengthen employees' competency, usefulness of the SoC theory in workplace health promotion, leadership, positive work experiences and possibility of recovering requirements. This section explores the health and well-being promoting factors of the workforce through the strongest themes emerging from the synthesis (acknowledging that in doing so, other aspects of health promoting of workforces may not be represented).

\subsubsection{Recovery in the workplace}

The following citations demonstrate the need for recovery of employees in the workplace:

\footnotetext{
"The ability to distance from work minimized the thoughts on work in the spare time, which was also important for being re-energized." (Nilsson et al., 2012).

"Recovery, measured as a combination of recovery during working hours and outside work, had the highest relationship to respondents' perceived health status." (Ejlertsson et al., 2018).
}

The studies of the review demonstrate that the possibility of employees to recover in the workplace is an important factor regarding health and well-being promotion of workforces. "Even employees with long working hours have been shown to have more need for recovery from work." (Ejlertsson et al., 2018, p. 159). It has also been shown that when there is insufficient recovery during working hours, the need for post-employment recovery is particularly important. Therefore, the 
synthesis suggests that enterprises should offer the possibility to relax in the workplace. As Nilsson et al. (2012) perceive that the time to chat about work, the workplace and family matters with fellow co-workers is important in order to savour positive experiences for use in times of adversity. Therefore, the synthesis suggests that employees should have the possibility to distance themselves from emotionally trying situations in the workplace and not only after work.

\subsubsection{Providing a resourceful working environment}

The second factor which the synthesis suggests, dominates the health and wellbeing promoting factors of workforces in the context of resources providing in the workplace. The research synthesis indicates that health and well-being of the workforces is more given if employees are provided with job resources because job resources build employees' SoC over time.

\footnotetext{
"When employees are provided with resources, a gain cycle may be initiated. The presence of job resources builds employees' SoC over time, making it more likely that those employees will activate and create even more resources in their environment". (Vogt et al., 2016, p. 11).

"Resources such as physical, mental, and social conditions of the workplace served a purpose of central nature to the employees". (Nilsson et al., 2012; p.163).
}

These finding are also important for salutogenic theory because it shows how SoC can be influenced and developed by working conditions (Vogt et al., 2016). Discussion about workplace health promotion, so the synthesis recommends, often takes place in correspondence with resource-orientation of WHP.

"In line with a call for positive health the construct of Work-SoC emphasises a resourceoriented view on workplace health promotion". (Vogt et al., 2013, p. 7).

Current WHP research suggests focus on resources within the workplace rather focusing on disease symptoms. The positive and no longer the negative side of 
health promotion is researched which corresponds to the character and approach of my study and correspond to the field of 'Positive Psychology'. More attention should be centered on developing and increasing positive psychosocial experiences while simultaneously indirectly reducing the negative (Saksvik et al., 2015). A resourceful working environment can be helpful in mastering difficult working conditions with resources 'resistance to change' and 'involvement' (Saksvik et al., 2015).

\subsubsection{Positive work experiences}

The synthesis suggests the factor 'Positive work experiences' as for example to be able to come to work with joy and to experience work as meaningful and challenging. The following citations of final studies display the importance of positive experience of employees within their workplace:

\footnotetext{
"Sense of usefulness; Relational quality: wanting the best for each other; Mastery; Zest for work. Experiences of being useful are identified as the most significant, however, all four experiences seem to stimulate each". (Vinje et al., 2013, p. 890).

"In the current study, the third significant variable connected to self-rated health was work experiences". (Ejlertsson et al., 2018, p.160). Therefore, "a good work environment has positive effects on the individual, their feelings about being useful, competent and enthusiastic [...]." (Ejlertsson et al., 2018, p. 160).
}

Employees can collect the positive experiences when they feel that they are needed, as the synthesis confirms. In other words, employees should have a feeling to be competent and to be useful. The requirements within the workplace should not exceed the competencies, as the final studies show. However, there are overlaps and similarities in the second and third factors of synthesis (providing a resourceful working environment and positive work experiences) that have been detected by the synthesis. Thus, resource-orientation in WHP seems to be related 
to positive experiences of employees, and therefore promote the health and wellbeing of employees.

\subsubsection{Strengthen employees' competency}

The synthesis suggests that health and well-being promotion in the workplace has to pay attention if they strengthen employees' competency.

"The competency to explore, note and understand the characteristics is yet another prominent finding in our study. The competency consists of the elements introspection, sensibility, reflection, and action/active mastery of the situation". (Vinje et al., 2013, p. 894).

In terms of strengthening competencies of employees, the synthesis shows that capacity in terms of participation and the willingness to change are important competencies for employees to stay healthy in the workplace which has to be strengthened by companies.

"[...] the content of the questions suggested that factors like 'resistance to change' and 'involvement' can be developed as more robust factors". (Saksvik et al., 2015, p.12-13).

The synthesis clearly highlights that employees stay healthy, if they strengthen their competencies and have the ability to shape and decide within their workplaces.

"Employees should act as creators of upcoming situations. Participation in questionnaire processes could bring positive qualities, such as empowerment, motivation, and team spirit, for the employees in workplace health promotion". (Nilsson, 2010, p. 11).

\subsubsection{Usefulness of the SoC theory in workplace health promotion}

The following citations demonstrate the usefulness of the SoC theory in WHP: 


\begin{abstract}
"In workplace health promotion is it therefore important to be attentive to work factors and processes that could contribute to employees' SoC, and to work with the experience of stressors in a positive sense rather than just a negative one". (Nilsson et al., 2012, p.166).

"At a more general level, the present results underlined the existence of a strong link between SoC and indicators of well-being in accordance with the central hypothesis of SoC theory [...]". (Feldt et al., 2000, p.472).

"Our stated hypothesis, that the SoC theory is useful in workplace health promotion, has been underpinned, first by the study findings that show the usefulness of SoC components when exploring context-specific resources considerable to the employees' health. Thus, SoC could also be useful for the employer when giving cause for promoting workplace health. Second, the SoC components could probably also be useful as a framework or starting point for discussing concrete work-related factors and processes, to increase the employees' understanding of what could contribute to their increased SoC". (Nilsson et al., 2012, p.164).
\end{abstract}

The synthesis of the final studies shows that the SoC theory presents an opportunity to explore, understand, and foster workplace resources (Nilsson et al., 2010). Salutogenesis works in current WHP research as an assistance by focusing on positive and resource-orientated factors rather on negative or pathogenic factors. With the basis of salutogenesis in WHP, health promotion factors also do justice to the well-being of employees (Nilsson, et al., 2010) and lead to a new way of thinking in terms of WHP. Salutogenesis, as the review shows, is flexible and can be implemented at different places. It seems that salutogenesis is the most important theory of health promotion so that models of WHP connect with it and/or use it as a basis regarding WHP. According to the final studies the use of salutogenesis in WHP shows success when it comes to the health and well-being of employees. For further requirements, research needs to apply the SoC theory into health and well-being promoting interventions of SMEs. 


\title{
3.8.6 Leadership
}

The theme 'leadership' is a present factor in the final studies and, therefore, the synthesis suggests that leaders should pay attention to their action and attitude, the involvement of employees and the creation of a pleasant work atmosphere (Saksvik et al., 2015). The following citations demonstrate the important factor of leadership regarding promotion of employee health and well-being:

\footnotetext{
"In this study, we found that good implementation of the intervention involving leadership role and communication also predicted levels of participants' satisfaction with this kind of salutogenic intervention program". (Saksvik et al., 2015, p.16-17).
}

\footnotetext{
"One conclusion is that it is essential for managers to support a work-life balance among the employees to create healthy work environments in a health care organization". (Ejlertsson et al., 2018, p.160).
}

\begin{abstract}
"An essential factor when creating a healthy work environment was that the manager made sure that the employees enjoy their work in a pleasant (fun) atmosphere". (Ejlertsson et al., 2018, p.160).
\end{abstract}

On the one hand, leadership plays an important role in fulfilling the needs of employees at the workplace and, on the other hand, they should play an active role in implementing WHP initiatives.

\footnotetext{
"The leader's role is to lead such a process and, by scrutinising, discovering and understanding, to improve her own practice as a leader". (Vinje et al., 2013, p. 899-900).
}

Successful WHP programmes need, besides other aspects, 'organizational actors' (Saksvik et al., 2015) and reflection leaders. Vinje et al. (2013) suggest structures be put into place that would enable the possibility for mutual reflection of leaders and their employees. Leadership is accorded an important role in synthesis when it comes to the successful implementation of WHP. 


\subsection{Summary}

The management field is seeking to tackle the complexity caused by the implementation of health and well-being promotion strategies of workforces, through a reliable basis of evidence and is helping to develop appropriate factors or initiatives and to take action. One reason for complexity is the increased requirements for workplace conditions with respect to employee health and employee well-being. Key sources of evidence, to identify health and well-being factors in SMEs include research on health and well-being promoting initiatives and the perspective for strategies for SMEs to promote employee health and wellbeing. Quality research supports access to these sources of evidence, through studies for health and well-being promoting factors of workforces, and their needs and perspective for SMEs' top management to implement health and well-being orientated factors. Until today, qualitative studies on how employee health and well-being in terms of salutogenesis can be promoted, occupy only a small area in the evidence base. It is quantitative studies assessing the effects of health orientated factors of employees which provide the main source of evidence. Research is also often done in nursing or hospital settings. Research on SMEs in relation to WHP in terms of salutogenesis cannot be found yet. No systematic review has yet been conducted on health and well-being promoting factors for workforces of SMEs in terms of salutogenesis and this systematic review is developed to fill this gap. It provides the first systematic review of qualitative research, in order to identify enablers to successfully implement health and wellbeing factors of workforces of SMEs. The review included studies from Europe, USA and Australia published from 1996, identified through comprehensive search strategies and quality assessed using established quality criteria. It showed that there is little evidence on how SMEs became successful in implementing WHP in terms of salutogenesis to promote employee health and well-being. Furthermore, it expressed the need for a qualitative study focusing on well-being or mental 
health and physical health with a positive and resource-oriented view like the theory of salutogenesis does.

The synthesis of this review followed four steps. First, I developed a theory of how the intervention works, why and for whom with the tool 'vote counting'. I created a preliminary synthesis with the help of a textual summary of the final studies which called 'The consideration of salutogenesis, positive working experiences and the resource view in WHP shows positive effects on the health and well-being of employees'. The preliminary synthesis of this review is close to the final synthesis. For the next step 'Exploring relationships within and between studies' I used the techniques of 'cross-case thematic analysis' for drawing out the main themes of the studies and technique 'Lines-of-argument synthesis'. The first stage of the techniques of 'cross-case thematic analysis' involved the identification of issues which are repeatedly highlighted in the studies (recovery in the workplace, providing a resourceful working environment, positive work experiences, strengthen employees' competency, usefulness of the SoC theory in WHP and leadership). While of potential theoretical interest, the review focused on the second stage of the synthesis which provides greater insight into health and wellbeing orientated factors in terms of salutogenesis. This is because the second stage of the synthesis enabled me to identify a broader and underlying set of experience, within and across the studies. An important underlying set of final studies show that WHP is geared towards the resources and needs of employees. Therefore, the workplace should be designed in such a way that employees can make good experiences and where their skills could be strengthened. The resource and needs oriented approach in WHP should be lived by top management and their leaders as the synthesis suggests at this stage. The last step of synthesis ('assessing the robustness of the synthesis') demonstrates that the final studies give important and relevant information regarding the review question and the study research questions. Moreover, they show validity by their methodology checked by the appraisal system of the quality assessment criteria 
(see chapter 3.5). Eight of ten studies are related to the research objectives of this study and show similarities with the nine factors of the study by Bruch et al. (2013) (see table 1) in their themes.

The chapter 3.7 'Discussion of final studies' presents the common concepts of final studies that have been found through the four synthesising steps of the study. Even though the studies apply the theory of salutogenesis differently it can be summarised as a helpful theory in WHP that focuses on humans, takes their needs into account and therefore shows positive effects in WHP. Hence, this review shows the trend in WHP towards more humanity with the implementation of the theory of salutogenesis and demonstrates it has not arrived in SMEs yet. The main goal of this review is to find answers for the review research question called: 'Which salutogenic factors support the promotion of health and well-being of the workforce in SMEs?' because it is necessary to know what are the interventions of WHP in terms of salutogenesis and to know which significant factors relate to implementation of WHP in terms of salutogenesis to support SMEs for future challenges. Some studies did not collect their data in the context of SMEs or did not inform about company size. They have taken place in nursing or hospital settings (as mentioned before) they can nevertheless provide important information for the health and well-being promotion in SMEs. Nevertheless, the review findings with respect to my research findings must consider this aspect of different settings. The synthesis of this review suggests the following salutogenic factors which support the promotion of health and well-being of workforces in SMEs: 


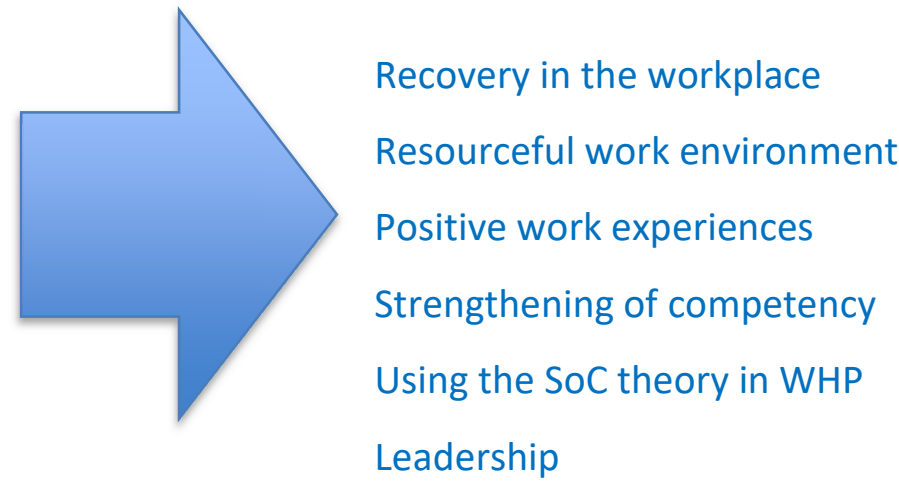

For further requirements research needs to concretely apply salutogenic criteria of Antonovsky's theory of SoC into SMEs practice. Qualitative research based on salutogenesis can search for deeper findings e.g. feelings, opinions and bias of manager and their employees in this field. Building on the issues highlighted in this review, it makes sense to note the need for qualitative studies on successful health and well-being orientated factors for workforces in SMEs as a specific contribution to qualitative studies on employee health and well-being promotion. Experiences of practical implementation of the SoC theory in SMEs are necessary. The next chapter displays the findings of the focus-group discussions using the ATA. 


\section{Findings of the focus-group discussions using the Applied Thematic}

\section{Analysis}

This chapter begins with a description of the research population and specific characteristics of the participants followed by the presentation of the data and discussion of the findings from the data generated. The results of the ATA provided categories for themes that led to answering the study research questions (see chapter 1.7). The aim of this chapter is the presentation and explanation of the study findings which I will discuss and analyse in connection with the factors of the study by Bruch et al. (2013), the SoC criterion of Antonovkys' salutogenesis and the three study research questions.

The following figure shows the chosen phases of the ATA recommended by Braun and Clark (2006) and Guest et al. (2012) where I orient myself. The different steps of 'Familiarisation with the data', the 'Coding' in NVivo, the 'Searching for themes' with the development of a codebook, 'Process for extracting codes and themes from focus-group discussions' is already done in the background.

Figure 15: Phases of the process of thematic analysis (own figure, content adapted from Braun \& Clarke (2006)).

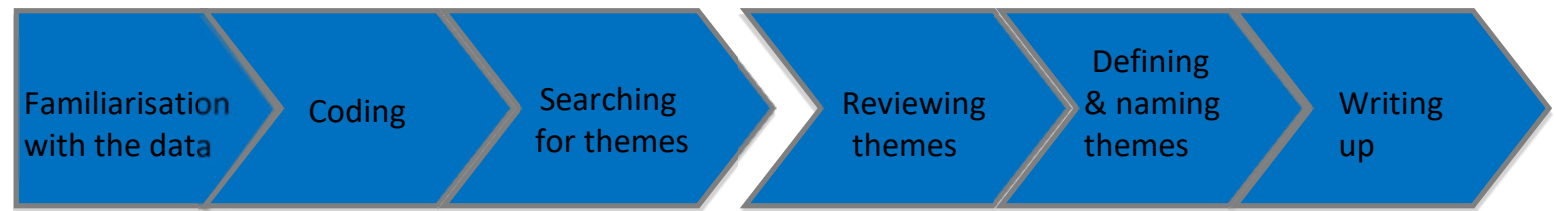

$\perp$

'Reviewing themes', the phase 'Defining and naming themes' and the 'Writing up' in the subchapter 4.4 'Findings answering the Research Questions' follows. At the end of this chapter, I assign the study findings to the three study research questions (see chapter 1.7 ) because they are used to provide relevant answers in order to support SMEs in EWL/Germany in salutogenic WHP. 


\subsection{Overview of general information about focus-group members}

This subchapter gives a short overview of the research population, the number, and structure of focus-group members chosen since the participants of the focus groups were already presented in chapter 2.5.1.

Out of 12 potential focus-group members who fitted the selection criteria (see chapter 2.5.1), I accept 12 requests for group discussion and I conduct 11 (one failed to attend) as mentioned in chapter 2.5.1 (see table 5).

The gender of identified focus-group members is unequally distributed. Three of the 11 participants are female, while eight are male. They are between 28 and 65 years of age and are all native German speakers.

All the chosen top managers have long-term experience in leading businesses of SMEs. Another reason to choose these leaders is that I know that they are open minded generally and interested in health promoting factors for SMEs.

Two of the five top managers own and lead SMEs in EWL/Germany. Three are salaried general or senior managers. Two SMEs are placed in the manufacturing industry and the other is placed in the tradesman business. The participating SMEs have from twelve to 220 employees. They have no integrated CHM system, but some have a little experience in WHP. The second group consists of six employees (two employees from each SME).

\subsection{Reviewing and defining potential themes}

I base the ATA on the themes identified in the focus-group analysis (see chapter 2.6). The following table is as well the basis of focus-group discussion analysis within NVivo and shows the themes identified in the focus groups. 
An exploration of salutogenic factors promoting workforce health and well-being in small and medium-sized businesses in East-Westphalia Lippe/Germany

Table 18: Tree of themes within this research project (a total of 11 participants).

\begin{tabular}{|c|c|c|}
\hline & Range of subjects/questions of the FGD & Themes as codes \\
\hline RQ 1 & $\begin{array}{l}\text { Q1 } \\
\text { Top management as pioneers } \\
\text { of a healthy performance culture }\end{array}$ & $\begin{array}{l}1 \text { Boss as pioneer } \\
\text { Boss_Pion } \\
2 \text { Boss should push the things } \\
\text { Boss_push }\end{array}$ \\
\hline RQ 1 & $\begin{array}{l}\text { Q2 } \\
\text { Healthy self-management }\end{array}$ & $\begin{array}{l}3 \text { Break } \\
\text { Bre }\end{array}$ \\
\hline RQ 1 & $\begin{array}{l}\text { Q3 } \\
\text { Freedom with a safety net }\end{array}$ & $\begin{array}{l}4 \text { Employee health vs. customer request } \\
\text { Empl_health_cust } \\
5 \text { Self-responsibility of employees } \\
\text { Self_resp_empl }\end{array}$ \\
\hline RQ 1 & $\begin{array}{l}\text { Q4 } \\
\text { Meaningful work }\end{array}$ & $\begin{array}{l}6 \text { Possibility of employees to learn about other areas } \\
\text { Pos_oth_areas } \\
7 \text { Possibility to place their own work into the bigger } \\
\text { context Pos_big_con }\end{array}$ \\
\hline RQ 1 & $\begin{array}{l}\text { Q5 } \\
\text { Value }\end{array}$ & $\begin{array}{l}8 \text { Importance of appreciation } \\
\text { Get_appre } \\
9 \text { Importance of praise } \\
\text { Prais_imp }\end{array}$ \\
\hline RQ $1 \& 3$ & $\begin{array}{l}\text { Q6 } \\
\text { Challenging and supporting }\end{array}$ & $\begin{array}{l}10 \text { Corporate culture } \\
\text { Coop_cult }\end{array}$ \\
\hline RQ 1 & $\begin{array}{l}\text { Q7 } \\
\text { Holistic health management }\end{array}$ & $\begin{array}{l}11 \text { Executive Training } \\
\text { Exec_train }\end{array}$ \\
\hline RQ 1 & $\begin{array}{l}\text { Q8 } \\
\text { "Health consumption" }\end{array}$ & \\
\hline RQ 1 & $\begin{array}{l}\text { Q9 } \\
\text { Psychological “immune system” }\end{array}$ & \\
\hline RQ $2 \& 3$ & $\begin{array}{l}\text { Q10 } \\
\text { Further measures \& ideas }\end{array}$ & $\begin{array}{l}12 \text { Recommendations: SMEs strengthen the sense of } \\
\text { togetherness } \\
\text { Sen_together } \\
13 \text { Recommendations: Measures more individual } \\
\text { Measure_indiv }\end{array}$ \\
\hline RQ $2 \& 3$ & $\begin{array}{l}\text { Q11 } \\
\text { What the FGD members take into the practice? }\end{array}$ & $\begin{array}{l}14 \text { Recommendations: Human being more important } \\
\text { than numbers } \\
\text { Human_impo }\end{array}$ \\
\hline
\end{tabular}

In the next step I summarise most of the themes into a range of topics displayed in the following figure: 
Figure 16: Overview of outstanding topics.
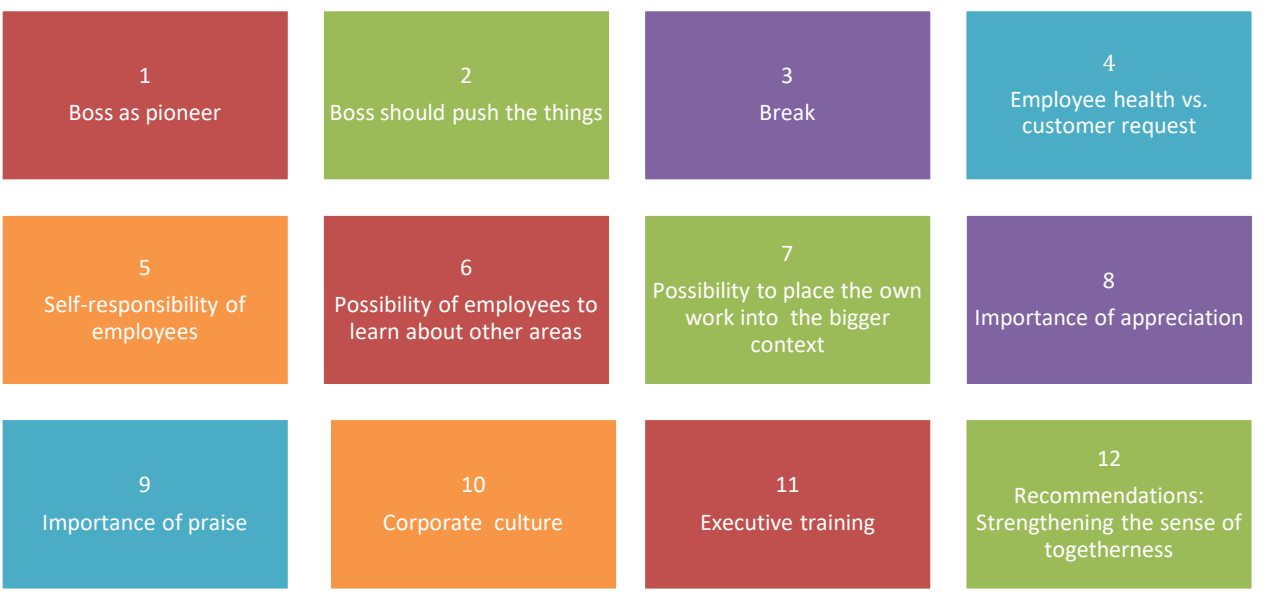

13

Recommendations:

Measures more individual

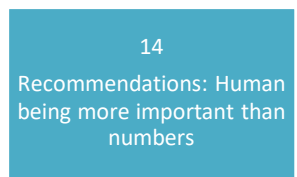

In the next step, I check the outstanding topics if they have potential to give important information for answering the three study research questions (see also chapter 1.7):

1. What salutogenic factors can be generated by top management to support the promotion of health and well-being of the workforce?

2. How can SMEs develop top management awareness to keep their staff healthy?

3. How can SMEs in East-Westphalia Lippe/Germany integrate salutogenic factors to promote the health and well-being of their workforces?

In addition to the relevance of the study research questions, I also ask whether the topics provide information on how to strengthen the $\mathrm{SoC}$ among employees. In other words, I link the 14 themes to the relevance of the SoC theory and check whether the themes contribute to the SoC criteria (comprehensibility, manageability and meaningfulness). It is also important that I consider which factors of the study by Bruch et al. (2013) are found or which are not found in the 14 themes. Finally, it must examine whether the themes are relevant in relation to 
the review findings. The following table assists me in reviewing the 14 themes on the above mentioned relevances:

Table 19: Themes checked regarding relevance.

\begin{tabular}{|c|c|c|c|c|c|c|c|}
\hline Theme & $\begin{array}{l}\text { Link to } \\
\text { SoC }\end{array}$ & $\begin{array}{l}\text { Promote which } \\
\text { SoC criteria }\end{array}$ & $\begin{array}{l}\text { Relevance } \\
\text { to } 1 . \mathrm{RQ}\end{array}$ & $\begin{array}{l}\text { Relevance } \\
\text { to 2. RQ }\end{array}$ & $\begin{array}{l}\text { Relevance } \\
\text { to } 3 . \mathrm{RQ}\end{array}$ & $\begin{array}{l}\text { Relevance } \\
\text { to the } \\
\text { study by } \\
\text { Bruch }\end{array}$ & $\begin{array}{l}\text { Relevance } \\
\text { to review } \\
\text { findings }\end{array}$ \\
\hline Boss as pioneer & $\checkmark$ & meaningfulness & $\checkmark$ & $\mathrm{x}$ & $x$ & $\checkmark$ & $\checkmark$ \\
\hline $\begin{array}{l}\text { Boss should push } \\
\text { the things }\end{array}$ & $\checkmark$ & manageability & $\checkmark$ & $x$ & $\mathrm{X}$ & $\checkmark$ & $\checkmark$ \\
\hline Break & $\checkmark$ & meaningfulness & $\checkmark$ & $x$ & $X$ & $x$ & $\checkmark$ \\
\hline $\begin{array}{l}\text { Employee health } \\
\text { vs. customer } \\
\text { request }\end{array}$ & $\mathrm{X}$ & $x$ & $\checkmark$ & $\mathrm{X}$ & $X$ & $\checkmark$ & $\checkmark$ \\
\hline $\begin{array}{l}\text { Self-responsibility } \\
\text { of employees }\end{array}$ & $\checkmark$ & $\begin{array}{l}\text { comprehensibility, } \\
\text { manageability, } \\
\text { meaningfulness }\end{array}$ & $\checkmark$ & $x$ & $x$ & $\checkmark$ & $\checkmark$ \\
\hline $\begin{array}{l}\text { Possibility of } \\
\text { employees to } \\
\text { learn about other } \\
\text { areas }\end{array}$ & $\checkmark$ & comprehensibility & $\checkmark$ & $\mathrm{X}$ & $x$ & $x$ & $\checkmark$ \\
\hline $\begin{array}{l}\text { Possibility to } \\
\text { place the own } \\
\text { work into the } \\
\text { bigger context }\end{array}$ & $\checkmark$ & $\begin{array}{l}\text { comprehensibility, } \\
\text { meaningfulness }\end{array}$ & $\checkmark$ & $\mathrm{X}$ & $x$ & $\checkmark$ & $\checkmark$ \\
\hline $\begin{array}{l}\text { Importance of } \\
\text { appreciation }\end{array}$ & $\checkmark$ & meaningfulness & $\checkmark$ & $x$ & $\mathrm{X}$ & $\checkmark$ & $x$ \\
\hline $\begin{array}{l}\text { Importance of } \\
\text { praise }\end{array}$ & $\checkmark$ & meaningfulness & $\checkmark$ & $\mathrm{x}$ & $x$ & $\checkmark$ & $x$ \\
\hline Corporate culture & $\checkmark$ & $\begin{array}{l}\text { comprehensibility, } \\
\text { manageability, } \\
\text { meaningfulness }\end{array}$ & $\checkmark$ & $x$ & $\checkmark$ & $\checkmark$ & $x$ \\
\hline Executive training & $x$ & $x$ & $\checkmark$ & $x$ & $x$ & $x$ & $\mathrm{X}$ \\
\hline $\begin{array}{l}\text { Strengthening } \\
\text { the sense of } \\
\text { togetherness }\end{array}$ & $\checkmark$ & $\begin{array}{l}\text { comprehensibility, } \\
\text { manageability } \\
\text { meaningfulness }\end{array}$ & $x$ & $\checkmark$ & $\checkmark$ & $x$ & $x$ \\
\hline $\begin{array}{l}\text { Measures more } \\
\text { individual }\end{array}$ & $\checkmark$ & $\begin{array}{l}\text { comprehensibility, } \\
\text { manageability, } \\
\text { meaningfulness }\end{array}$ & $x$ & $\checkmark$ & $\checkmark$ & $\checkmark$ & $x$ \\
\hline $\begin{array}{l}\text { Human being } \\
\text { more important } \\
\text { than numbers }\end{array}$ & $\checkmark$ & $\begin{array}{l}\text { comprehensibility, } \\
\text { meaningfulness }\end{array}$ & $x$ & $\checkmark$ & $\checkmark$ & $\checkmark$ & $x$ \\
\hline
\end{tabular}


The examination revealed that most of the 14 themes have the potential to give important information regarding health and well-being promotion of workforces in terms of salutogenesis in SMEs. 'Employee health vs. customer request' and 'Executive training' show no direct link to SoC.

Of the remaining 12 themes, nine themes are similar or identical to the factors of the study by Bruch et al. (2013). This could mean that the focus groups rated the factors of the study by Bruch et al. (2013) as positive with respect to employees' health promotion and considered them to be practical for health promotion in SMEs. The focus group participants have generated new ideas for WHP in SMEs, such as 'Possibility of employees to learn about other areas' or 'Strengthening the sense of togetherness', which help to answer the three research questions of my study. Furthermore, the comparison reveals that some potential themes gathered from focus groups (e.g. 'Boss as pioneer') deal with the role of the leader and thus can be related to the review finding 'Leadership'. The themes 'Boss as pioneer' and 'Boss should push the things' are further used as potential themes in this study. But since there are two similar themes, I summarise them in one theme named 'Boss as pioneer and initiator'. The comparison also shows that there are further themes which are similar. So, the themes 'Importance of appreciation', 'Importance of praise' and 'Human being more important than numbers' is summarised to the theme 'Appreciation by praise'. The theme 'Possibility of employees to learn about other areas' and the theme 'Possibility to place the own work into the bigger context' are also summarised to one theme: 'Possibility to learn about other areas and to place the own work into the bigger context'. The following potential themes emerge after checking for relevance and summarising: 


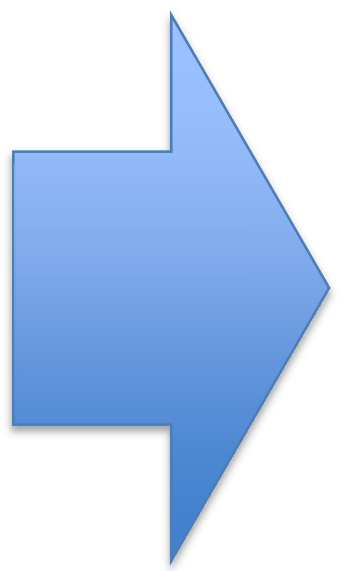

Boss as pioneer and initiator

Appreciation by praise

Break

Possibility to learn about other areas and to place the own work into the bigger context

Self-responsibility of employees

Corporate culture

Strengthening the sense of togetherness

Measures more individual

The eight themes gathered from the two focus groups are classed with codes and described in the codebook (see appendix 9.9) for analysis. The following section shows the importance of the theme 'Boss as pioneer and initiator' and the theme 'Appreciation by praise' which is discussed most intensively and several times in the focus groups by using citations of participants.

\section{Boss as pioneer and initiator}

The first slide (see appendix 9.11) 'I am a pioneer as a boss', which represented the first measures of Mr. Müller, is discussed extensively by both focus groups, especially from the top managers. During the focus-group discussion the theme 'Boss should push things' also took up space by the group of the employees. The role of a trendsetter brought a discussion point: the proximity of employee and top manager in SMEs:

"[...] but I think what we have in common is the role model function, which is in both areas, large and small - the proximity makes the perception, I think, different."

In the discussion of the top managers on this topic, the importance of the special closeness that top managers in SMEs have to their employees is discussed. Also, 
the top managers added that top manager need multipliers which also play the pioneer role:

"[...] that's actually derived a bit from the fact that we say, 'not just the owner leads by example', but he also needs multipliers."

"[...] the boss at the top should not have the pioneering role [...], but that would really

[....] be broken down on the executives."

With the topic of the trendsetting role, the top managers also discussed the topic of responsibility. They emphasised that responsibility lies also with the other executives of the company. Two top managers mentioned the limit of their responsibility:

"[...] there is a limit, and the limit is that everyone is responsible for themselves at the end of the day, to get well, to do sports, to eat healthily, and so on, we quickly come in the direction: The company is responsible for the health."

"Whereby I find the viewpoint [...] of this double image, exciting [...] on the one hand role, yes, we have a role, but on the other hand self-responsibility."

In the employee group the topic 'boss as a pioneer' is given more importance than in the group of top managers. Three employees emphasised the importance of the role model and trendsetter role of a top manager regarding health promotion:

"[...] to take over a certain role model on the topic of health is certainly beneficial in my view, only the crux lies in convincing the staff [...]."

"So, the supervisor as a pioneer, clear, [...] maybe even one with the most important points."

"[...] it is also the case that the boss is actually operational around the clock, ready for action, and I find that this already transfers [...] to the employees."

On the other hand, some employees have expressed their opinions critically and are not sure whether the top manager' role model image is perceived by the employees:

"I do not think so in terms of themselves, so it's like that with us, our two top manager are coming to work by bike most of the time, but it's the top manager who come by bike to work, but to transfer that to itself, I do not think so automatically takes place." 
"I also do not think many employees would perceive what the boss is doing because they are way too far away - whether they come by bike or had salad for lunch. [...] because probably there is such a distance between them."

One employee has pointed out that the employer should give stimulus regarding health promotion:

"You would have to somehow give the employees a suggestion or something that they just have to deal with, even during working hours [...]."

All focus-group employees recommended SMEs to show possibilities to the employees regarding WHP during the working hours. They emphasised the employer should push WHP initiatives in the first step.

\section{Appreciation by praise}

The theme 'Get appreciation' of the fifth slide ('Value', see appendix 9.11) is discussed extensively by the focus group of employees. The group of the top managers has discussed the theme ,Praise is important' which is very similar in theme.

Two employees have noted:

"I think that one is appreciated, but it does not come over from above. There is more from the customers."

"Must be easy in between to say, "Man, have you done well," or "Great, I' am happy." It just sometimes comes too little."

Employees have repeatedly stated at various points in the focus discussion that they receive too little appreciation. The recognition of their work and the positive feedback come too short. Motivation in the work by appreciation of the boss is also discussed in this context. Both topics seem to go hand in hand with the staff:

\footnotetext{
"[...] at the end of the day you are always just a number in the company. And if then always my commitment is ultimately valued in the sense? [...] I always motivate myself very much. So that I have fun at work, and that I am committed to it and now not so very high, that also an appreciation, because I know, in the big sense, there is not that, but then I prefer to go in myself and motivate myself. "Would be good if there's a bit more
} 
from the top, if the customer company has called, we are successfull and everything is fine."

An employee with top managerial responsibility has mentioned:

"I can motivate myself and I also feel valued, but I also noticed as a leader, not every employee can deal with an appreciation [...]. Then there are sometimes questions such as: "The praise that you have given me now, did you mean that seriously now or are you kidding me now?"

The other employees have noticed:

\footnotetext{
"Yes, one rarely hears this so much that one does not even know how to handle it."

"I still think appreciation is one of the most important points [...]. "

"So, I would rather say that I create a sensible culture of appreciation and then build around it the story of the holistic health system."
}

The problem that employees find appreciation alien is discussed but every one of the employee group agreed that a culture of appreciation (as part of a comprehensive health management $\mathrm{CHM})$ ) must be lived so that employees can accept positive feedback and are not be critical. It should be a matter of course in SMEs to regularly praise the staff according to the opinion of the employee focus group.

The following chapter presents the eight themes in summary with interpretations and their contribution to SoC and the factors of the study by Bruch et al. (2013).

\subsubsection{Summary and presentation of themes with their contribution to SoC and the factors of the study by Bruch et al. (2013)}

The theme 'Boss as pioneer and initiator' is discussed extensively by both focus groups, especially from the top managers. The role of a pioneer brought another discussion point regarding the proximity of employee and top manager in SMEs. In this regard, a top manager has mentioned "[...] I think what we have in common is the role model function, which is in both areas, large or small - the proximity 
makes the perception I think different". At this point, the difference between small and large companies is emphasised. In concrete terms, this could mean that employees of SMEs have advantages regarding health promoting, because the top manager is closer to the employees than in big companies. The topic of 'closeness between the boss and employees', which is also a subject of discussion elsewhere, showed the importance of the topic at this point. The top manager added that top managers need multipliers which play also the pioneer role "[...] that's actually derived a bit by the fact that we say, I have such a cascade, not just the owner leads by example, but he also needs multipliers". The top managers of the group seem to be generally aware of the role as responsible (even if they want to share responsibility with their managers), but the role as a trendsetter is foreign to them, as they do little to address that aspect. The top managers of the focus group know that their influence on the health of their employees is limited only if the employees themselves do not feel responsible. On the other hand, two employees have undertaken and have seen the responsibility also of the top manager or enterprise. They have emphasised that the role model as top manager is beneficial but only the crux lies in training the staff. It seems as if the role model of the boss is important for the health promotion of the employees because if the boss realises that it is important to take care of his health and, for example take care of work life balance, the employees dare to do so too. Otherwise employees have the feeling that it is not desired to care about their work life- balance and they have disadvantages. Some employees of the focus group are critical regarding the theme of role model of the boss. It seems that employees who are not close to the boss themselves may not perceive this aspect as important. Another assumption is that employees only identify with the boss' behaviour (riding a bike to work) if it seems manageable and meaningful for them.

During the focus-group discussion the theme 'Boss should push things' also took up space by the group of the employees. One employee pointed out that the employer should give stimulus regarding health promotion "You would have to somehow give the employees a suggestion or something that they just have to 
deal with, even during working hours [...]". Responsibility is thus projected also onto the company. Another employee added everyone is responsible for their own health but 'a support from the boss' should also be there. It seems important for a successful implementation of WHP initiatives that SMEs show a lot of commitment and interest in the health of employees, especially the boss should be engaged in this regard.

However, the theme 'Boss as pioneer and initiator' has to be seen in the context of small businesses where the top manager works close with his employees and communicate directly with them. In bigger companies all managers should be pioneers and initiators regarding WHP. In other words, this theme relates to a specific form and size of organisations which will I discuss in the last chapter.

Theme contribution to SoC

Theme contribution to Bruch et al.

\begin{abstract}
The theme 'Boss as pioneer and initiator' could strengthen the SoC factors 'meaningfulness', 'comprehensible' and 'manageability' of employees.
\end{abstract}

The theme 'Boss as pioneer and initiator' complies with a recommendation of the study by Bruch et al. (2013) ('Top management as pioneers of a healthy performance culture', see table 1) and is confirmed with my study for integration in SMEs in EWL/Germany to promote the health and well-being of employees.

The theme 'Get appreciation' is discussed extensively by the focus group of employees. The group of the top managers have discussed the theme ,Praise is important' which is very similar in theme (see appendix 9.11). One of the employees has noted that 'appreciation' does not take place. He said that there is more appreciation from the customer. Another employee confirmed the statement: "Would be good if there's a bit more from the top, if the customer company has called, we are successful, and everything is fine". An employee expresses the wish to get more appreciation like this: "Man, have you done well," or "Great, I am happy". One employee is aware that at the end of the day, he is 
only a number. Everyone has agreed that the appreciation by the top manager is important and one employee emphasised the importance of this measure again with the following assessment: "I still think appreciation is one of the most important points." As this topic has been discussed in many places of focus groups of employees, it is an important aspect in the promotion of health and the wellbeing of employees when they are valued by their boss. They need appreciation, as they do not feel like just a 'number' in the company. It seems strange for top managers of the focus group to praise employees on a regular basis. A notable experience of one top manager in this context convinced him of the importance of the praise of employees: "I had a guy with tears in front of me who had been with us for eight or nine years and I said," Man, your job is good, [...] we appreciate your work, stay cool, you make everything good and right, carry on." He said at that moment with tears in his eyes, that this was the first time that someone told him that he did something well. That's important for the people. I became very aware of this at that moment". Another top manager mentioned that it is hard for him hard to praise the one who just annoyed him.

Theme contribution to SoC

\section{The theme 'Appreciation by praise' could strengthen the SoC factor 'meaningfulness' of employees.}

The theme 'Appreciation by praise' complies with a recommendation of the study by Bruch et al. (2013) ('Value', see table 1) and is confirmed with my study for integration in SMEs in EWL/Germany to promote the health and well-being of employees.

The second slide (see appendix 9.11) which represented the measures of Mr. Müller regarding 'Healthy self-management' is discussed extensively by the top managers, especially the theme 'Break'. One top manager is open to innovative break design as he already experienced it in his professional environment: "An owner of a friendly electrical company takes a nap for half an hour - and he also 
pauses. I say, "What, you sleep", yes, he lies down. So that's how it is". Another top manager commented that the break can be individually designed: "Of course, everyone can use the break as he wants. One smokes, the other sleeps. And when you would have to provide beds, a dorm, because there is the demand, I think we would not mind". This statement showed that the employees themselves can make their breaks, but the company does not actively support design. This rather passive attitude of the top manager on this topic is discussed by another two top managers with the following statement: "[...] I find that unrealistic". One top manager added that the lunch time of 30 minutes is pretty tight already. So, the employees have to add the extended break on the working time. Discussions on this topic show that top managers do not feel responsible for making their employees' breaks healthy, although it is important for employees to have space and time for regeneration during work as the review of this study shows (Nilsson et al., 2012, Ejlertsson et al., 2018).

Theme contribution to SoC

Theme contribution to Bruch et al.
The theme 'Break' could strengthen the SoC factor 'meaningfulness' of employees.

The theme 'Break' does not meet the recommendations of the study by Bruch et al. (2013).

The fourth slide named 'Meaningful work' is discussed intensively by the employees regarding 'Possibility to learn about other areas' (see appendix 9.11). The importance became clear in the scope and statements on this subject also with this quote of an employee: "I think it's good. I've already done my teaching at [...], and that's why you just walked around the company, through all the departments. You know the people, you know what's being done here, what's being done, how is being produced [...]". Another employee confirmed the statement: "When I started at [...] I was urged to get to know the company, and then I was also given the opportunity to spend a week in the company because it 
was very important to me to get to know all the processes, yes which of course helps me a lot further in my daily work". An employee emphasised the importance of changing perspectives: "[...] only when he is over there and sees the problems that they have, then he also has a completely different perception of this story". It seems to be important for employees regarding their health and well-being that they know what impact their work has on other businesses of the company. Knowing how other areas of the company work, helps to understand the importance of their work and that seems to be important for the health and wellbeing of employees. In this context, the topic 'Possibility to place their own work into the bigger context' is discussed by the employees. In this regard one employee mentioned: "Yes, I think that's very important. So, if you do not see any meaning in your work, that would be the worst, the worst starting point. And if the top manager also communicates what they do for the company, where the contribution I make, where to put it, that would definitely be a good thing". Knowing the importance of their in the company and what it does to make the company a success seems to be important to employees.

Theme contribution to SoC

Theme contribution to Bruch et al.
The theme 'Possibility to learn about other areas and to place the own work into the bigger context' could strengthen the SoC factors 'comprehensibility' and 'meaningfulness' of employees.

\footnotetext{
The theme 'Possibility to learn about other areas and to place the own work into the bigger context' complies with a recommendation of the study by Bruch et al. (2013) ('Meaningful work', see table 1) and is confirmed with my study for integration in SMEs in EWL/Germany to promote the health and well-being of employees.
}

The focus group of the top managers discussed the point 'self-responsibility of employees'. One top manager noticed that his company offers WHP measures, 
but employees do not accept them: "[...] you can offer exercise programmes [...]. They still do not use exercise programmes, but they have at least a guilty conscience [...]". The issue of 'self-responsibility of employees' is discussed at other points in the group discussion and therefore made clear that it is important for the health and well-being of employees that they feel first and foremost responsible for their health. This is the only way SMEs can get their employees through WHP initiatives, as the findings of the focus groups show.

Theme contribution to SoC

Theme contribution to Bruch et al.
The theme 'Self-responsibility of employees' could strengthen the SoC factors 'comprehensibility', 'manageability' and 'meaningfulness' of employees.

The theme 'Self-responsibility of employees' complies with a recommendation of the study by Bruch et al. (2013) ('Health consumption', see table 1) and is confirmed with my study for integration in SMEs in EWL/Germany to promote the health and well-being of employees.

The sixth slide (see appendix 9.11) 'Challenging and supporting', is discussed extensively by top managers regarding the topic of 'corporate culture'. All top managers agreed that it is an important issue, which makes up the SMEs. A top manager noted that 'corporate culture' is always strongly shaped by the top manager, his personality and by a top managerial family. One top manager noted: "[...] I say to all employees, that's all of us, that is. So, everyone is a building block, but the imprint, that DNA, is of course also lived by the top manager, the manager family [...], I have to see that". A top manager has also added related to the mediation of corporate culture: "[...] that starts with the supporter and not just with the processing, where just [...] corporate culture is taught". The importance of 'Corporate Culture' is further highlighted by another top manager: "We also have to take the people with us, and that's one of the issues of health. And if you then put it into such an instrument and there is a role in health, then I think you 
have the routine, where that can be a kind of component of the corporate culture at the end". The intensive discussion on the topic shows that individual initiatives to promote employees' health fail but that they must be embedded in a CHM system of the company. For example, it makes more sense for employees to look after their health in a work context because it counts as a corporate spirit.

Theme contribution to SoC

Theme contribution to Bruch et al.
The theme 'Corporate culture' could strengthen the SoC factors 'comprehensibility', 'manageability' and 'meaningfulness' of employees.

The theme 'Corporate culture' complies with a recommendation of the study by Bruch et al. (2013) ('Holistic health management', see table 1) and was confirmed with my study for integration in SMEs in EWL/Germany to promote the health and well-being of employees.

The theme 'Strengthening the sense of togetherness' (see figure 9, 'further ideas and measures') is recommended by a top manager. One top manager mentioned it that way: "What we have determined, what we do - that is a bit 'feel good', but then also in the direction of movement and the transfer of various possibilities, there is also what needs to be done: to make joint events. I'm not really a fan of mixing work and private life. But what we have noticed is that we have a better mood in the company if we do something together on a regular basis". This theme shows that the top managers have learned that WHP is more than just "back training' but that events are also important where employees and managers can exchange views and gain positive experiences as a team outside of work. Strengthening employees' sense of belonging seems to be an important aspect in health and well-being promotion of employees in SMEs in EWL/Germany. 
Theme contribution to SoC

Theme contribution to Bruch et al.
The theme 'Strengthening the sense of togetherness' could strengthen the SoC factors 'comprehensibility', 'manageability' and 'meaningfulness' of employees.

The theme 'Strengthening the sense of togetherness' does not meet the recommendations of the study by Bruch et al. (2013).

The recommendation 'Measures more individual' (see figure 9 'further ideas and measures') was mentioned in the employee focus group. One employee has mentioned that the ideas and the points that Mr. Müller implemented are all good, but they have just to fit in with the company, so it has to be an individual solution. He has noted that measures tailored to the company and to the teams and where everyone can participate. WHP initiatives seem to be important for the individual needs of the staff or teams. For this purpose, it is necessary for SMEs in $\mathrm{EWL} /$ Germany to query the employees' assessments at regular intervals in order to be able to develop suitable initiatives or to adapt initiatives.

Theme contribution to SoC

\begin{abstract}
The theme 'Measures more individual' could strengthen the SoC factors 'comprehensibility', 'manageability' and 'meaningfulness' of employees.
\end{abstract}

The theme 'Measures more individual' complies with a recommendation of the study by Bruch et al. (2013) ('Holistic health management', see table 1) and is confirmed with my study for integration in SMEs in EWL/Germany to promote the health and well-being of employees.

This chapter shows that the eight themes based on my interpretations of the focus group data can strengthen the SoC of employees by responding to the SoC criteria (comprehensibility, manageability and meaningfulness). In other words, they bring 
the conditions to promote the SoC of employees in SMEs in EWL/Germany. Each theme has at least a connection to one SoC criterion, but most topics take two or even all SoC criteria into account.

In addition, five themes show that they have a connection to the recommendations within the study by Bruch et al. (2013). This means that the participants of the focus groups consider the recommendations within the study by Bruch et al. (2013) to be important for the health and well-being in SMEs in EWL/Germany. Five themes of the study by Bruch et al. (2013) have proven to be valuable in my focus groups and have already been discussed in chapter 1.6. (see figure 2) associated with the SoC criteria.

Three of the eight themes are recommendation of the focus-group participants and find no connection to the study by Bruch et al. (2013) but as mentioned before show connection to the SoC.

The introduced themes of this chapter and their contribution to SoC and their contribution to the study by Bruch et al. (2013) will be further discussed in the last chapter regarding contributions, limitations and further research. However, the current phase 'Writing up' (see figure 15) is not yet complete because I put the eight themes presented in this chapter in comparison to the themes that a reflection group has drawn from the focus-group data. The next chapter 4.3 presents further themes gathered by a group of experienced employees and employers which belongs to family and friends of mine. I named the group 'reflection focus group' because they maybe reflect and interpret the data of the two focus groups differently than I do, and either confirm or reject my interpretation of the data (see further explanation in the following chapter).

\subsection{Implementing a reflection focus group discussion}

One of the big challenges in evaluation of data generated is how to engage with the findings. 'How other people would analyse the data or are their conclusions 
more valid? etc. are some of the questions which arise before or during data analysis (B. Williams, 2017). Several methods are adopted to enhance validity, including triangulation, well-documented audit trail and multidimensional analysis etc. (Leung, 2015).

I choose a kind of multidimensional analysis by giving the research another dimension to analyse the data: a reflection focus-group discussion. It is mostly based on the model of Bob Williams (2017) who puzzled about this model for a few years. He came across some colleagues using ideas drawn from Vygotskyian (Wertsch, 1986) based on psychology and Activity Theory (Engeström, 1999). The model of Williams postulates that we learn from two different practices. Patterning (i.e. fitting current events into past events), and puzzling (i.e. seeking explanations why the current event doesn't fit into past events, or even other current events). Williams (2017) encourages to puzzle over the data rather pattern the data. The analysing process by Williams (2017) is based on identifying and discussing four aspects of the data: Generalisations and Exceptions (usually, but), Contradiction (on the one hand, on the other hand), Surprises (I'd expected, but) (I didn't expect, but) and Puzzle (Why?). I have followed this analysing process in the most points and have performed a reflection focus group discussion to achieve more objectivity in the data analysis process. Aim of the reflection-group discussion is how the members interpret the statements of the focus groups or do they interpret statements differently than I do. Do they find generalisations or contradictions?

Three members participate in the reflection focus group discussion. They are family and friends of mine, experienced employees or employers in SMEs, interested in the topic of WHP in SMEs and open-minded. The average age of the reflection focus group is 56 years and they are all female. This group is used to compare my interpretation of the data of the focus groups with those of the reflection group. 
I give the members a summary (see appendix 9.12) of the findings from the two focus-group discussions a few days before. So the members have time to read the summary in their own time and at their own pace.

The discussion is carried out in a private venue organised by me and snacks and drinks are provided. The reflection group is moderated by me and I lead through the charts representing the measures of the fictional top manager character Mr. Müller (see appendix 9.11).

The training as a coach, and the background of coaching experience, helps me to focus on the essential, so that there are no conflicts in the role as moderator and in presenting the results (as mentioned before). I behave as neutrally as possible, so that the members are encouraged to answer honestly and not simply give the desired answers. The discussion is recorded by an audio recording player and is transcribed by an agency. The audio data accumulate to nearly 2 hours of audio data, 29 DIN A4 pages of printed text and 12,837 words.

\subsubsection{Analyses and overview of findings of the reflection focus group}

I analyse the findings of the reflection discussion group manually because the amount of data is not as extensive as the data of the focus groups of top managers and their employees. There are only a few criteria of interest by analysing the data so that I do not need the software NVIVO. I search specifically for the following points in the text analysis:

- interpretation of focus-group statements different from mine

- if generalisations can be drawn from the statements of the focus groups

- $\quad$ if there are any contradictions.

In the first step, I gather the evaluated themes of the reflection group in the following table. Afterwards, I compare the themes of the reflection group with my themes and decide if they provide important new information or confirm my 
analysed themes. The following table shows the themes interpreted by the reflection focus group (see fourth column) in connection with my interpretation of themes:

Table 20: Presentation of themes interpreted by me and by the reflection group.

\begin{tabular}{|c|c|c|c|}
\hline & Range of subjects/questions & Topics interpreted by me & Topics interpreted by reflection group \\
\hline RQ 1 & $\begin{array}{l}\text { Q1 } \\
\text { Top management as pioneers } \\
\text { of a healthy performance culture }\end{array}$ & Boss as pioneer and initiator & $\begin{array}{l}\text { Boss alone is not enough } \\
\text { Boss_not_enough } \\
\text { Proximity of the boss } \\
\text { Proximity_boss } \\
\text { Combination of professional \& private } \\
\text { not wanted } \\
\text { Comb_not want } \\
\text { Boss' authenticity } \\
\text { Boss_auth }\end{array}$ \\
\hline RQ 1 & $\begin{array}{l}\text { Q2 } \\
\text { Healthy self-management }\end{array}$ & Break & $\begin{array}{l}\text { Healthy self-management is unfamiliar } \\
\text { and foreign for the focus group's top } \\
\text { managers } \\
\text { Healthy self ma_unfamiliar }\end{array}$ \\
\hline RQ 1 & $\begin{array}{l}\text { Q3 } \\
\text { Freedom with a safety net }\end{array}$ & $\begin{array}{l}\text { Self-responsibility of } \\
\text { employees }\end{array}$ & $\begin{array}{l}\text { Top managers of focus group try to } \\
\text { make a good impression } \\
\text { Try_good_imp }\end{array}$ \\
\hline RQ 1 & $\begin{array}{l}\text { Q4 } \\
\text { Meaningful work }\end{array}$ & $\begin{array}{l}\text { Possibility to learn about } \\
\text { other areas and to place the } \\
\text { own work into the bigger } \\
\text { context }\end{array}$ & $\begin{array}{l}\text { Trust in the boss } \\
\text { Trust_to_boss }\end{array}$ \\
\hline RQ 1 & $\begin{array}{l}\text { Q5 } \\
\text { Value }\end{array}$ & Appreciation by praise & $\begin{array}{l}\text { Fear of costs of top manager of the focus } \\
\text { group } \\
\text { Fear_costs }\end{array}$ \\
\hline \begin{tabular}{lll|}
$R Q$ & 1 & $\&$ \\
3 & & \\
\end{tabular} & $\begin{array}{l}\text { Q6 } \\
\text { Challenging and supporting }\end{array}$ & Corporate culture & $\begin{array}{l}\text { Good contact with the boss } \\
\text { Good_cont }\end{array}$ \\
\hline \multirow[t]{3}{*}{ RQ 1} & $\begin{array}{l}\text { Q7 } \\
\text { Holistic health management }\end{array}$ & & $\begin{array}{l}\text { The big picture has not arrived by the } \\
\text { top managers yet } \\
\text { Not_arriv }\end{array}$ \\
\hline & $\begin{array}{l}\text { Q8 } \\
\text { ‘Health consumption’ }\end{array}$ & & $\begin{array}{l}\text { Criticism of data usage } \\
\text { Critic_data }\end{array}$ \\
\hline & $\begin{array}{l}\text { Q9 } \\
\text { Psychological 'immune system' }\end{array}$ & & $\begin{array}{l}\text { Longing for good old days } \\
\text { Long_old_days } \\
\text { Uncertainty because topic is too abstract } \\
\text { Uncert_too_abstr }\end{array}$ \\
\hline $\begin{array}{lll}\text { RQ } & 2 & \& \\
3 & & \\
\end{array}$ & $\begin{array}{l}\text { Q10 } \\
\text { Further measures \& ideas }\end{array}$ & $\begin{array}{l}\text { Strengthen the sense of } \\
\text { togetherness } \\
\text { Measures more individual }\end{array}$ & $\begin{array}{l}\text { Will is there } \\
\text { Will_there }\end{array}$ \\
\hline $\begin{array}{lll}\text { RQ } & 2 & \& \\
3 & & \\
\end{array}$ & $\begin{array}{l}\text { Q11 } \\
\text { What the FGD members take into } \\
\text { the practice? }\end{array}$ & & $\begin{array}{l}\text { Knowledge is missing } \\
\text { Know_miss } \\
\text { Concept of health promotion is } \\
\text { misleading } \\
\text { Conc mislead }\end{array}$ \\
\hline
\end{tabular}


In summary 16 themes are found by the members of the reflection group and are compared with my themes in the next step. For that I check (as well as for my themes, see table 21) whether the 16 themes provide relevant information regarding salutogenesis, the three study research questions and the nine factors of the study by Bruch et al. (2013).

Table 21: Themes of reflection group checked regarding relevance.

\begin{tabular}{|c|c|c|c|c|c|c|c|}
\hline Theme & $\begin{array}{l}\text { Link to } \\
\text { SoC }\end{array}$ & $\begin{array}{l}\text { Promote which } \\
\text { SoC criteria }\end{array}$ & $\begin{array}{l}\text { Relevance } \\
\text { to 1. RQ }\end{array}$ & $\begin{array}{l}\text { Relevance } \\
\text { to } 2 . \mathrm{RQ}\end{array}$ & $\begin{array}{l}\text { Relevance } \\
\text { to } 3 . \mathrm{RQ}\end{array}$ & $\begin{array}{l}\text { Relevance } \\
\text { to the } \\
\text { study by } \\
\text { Bruch et } \\
\text { al. (2013) }\end{array}$ & $\begin{array}{l}\text { Relevance } \\
\text { to review } \\
\text { findings }\end{array}$ \\
\hline $\begin{array}{l}\text { Boss alone is not } \\
\text { enough }\end{array}$ & $\mathrm{x}$ & $x$ & $x$ & $x$ & $x$ & $x$ & $x$ \\
\hline $\begin{array}{l}\text { Proximity of the } \\
\text { boss }\end{array}$ & $\checkmark$ & comprehensibility & $\checkmark$ & $x$ & $x$ & $x$ & $x$ \\
\hline $\begin{array}{l}\text { Combination of } \\
\text { professional \& } \\
\text { private not } \\
\text { wanted }\end{array}$ & $x$ & $x$ & $x$ & $x$ & $x$ & $x$ & $x$ \\
\hline $\begin{array}{l}\text { Boss' } \\
\text { authenticity }\end{array}$ & $\checkmark$ & meaningfulness & $\checkmark$ & $x$ & $x$ & $\checkmark$ & $x$ \\
\hline $\begin{array}{l}\text { Healthy self- } \\
\text { management is } \\
\text { unfamiliar and } \\
\text { foreign for the } \\
\text { focus group's } \\
\text { top managers }\end{array}$ & $x$ & $\mathrm{X}$ & $x$ & $x$ & $x$ & $x$ & $x$ \\
\hline $\begin{array}{l}\text { Top managers of } \\
\text { focus group try } \\
\text { to make a good } \\
\text { impression }\end{array}$ & $x$ & $x$ & $x$ & $x$ & $x$ & $x$ & $x$ \\
\hline Trust in the boss & $\checkmark$ & meaningfulness & $\checkmark$ & $x$ & $x$ & $\checkmark$ & $x$ \\
\hline $\begin{array}{l}\text { Fear of costs of } \\
\text { top manager of } \\
\text { the focus group }\end{array}$ & $x$ & $x$ & $x$ & $x$ & $x$ & $x$ & $x$ \\
\hline $\begin{array}{l}\text { Good contact } \\
\text { with the boss }\end{array}$ & $\checkmark$ & $\begin{array}{l}\text { comprehensibility, } \\
\text { meaningfulness }\end{array}$ & $\checkmark$ & $x$ & $x$ & $\checkmark$ & $x$ \\
\hline $\begin{array}{l}\text { The big picture } \\
\text { has not arrived } \\
\text { by the top } \\
\text { managers yet }\end{array}$ & $x$ & $x$ & $x$ & $x$ & $x$ & $x$ & $x$ \\
\hline $\begin{array}{l}\text { Criticism of data } \\
\text { usage }\end{array}$ & $x$ & $x$ & $x$ & $x$ & $x$ & $x$ & $x$ \\
\hline $\begin{array}{l}\text { Longing for } \\
\text { good old days }\end{array}$ & $\checkmark$ & meaningfulness & $\checkmark$ & $\checkmark$ & $x$ & $x$ & $x$ \\
\hline $\begin{array}{l}\text { Uncertainty } \\
\text { because topic is }\end{array}$ & $x$ & $x$ & $x$ & $x$ & $x$ & $x$ & $x$ \\
\hline
\end{tabular}




\begin{tabular}{|l|c|c|c|c|c|c|c|}
\hline too abstract & & $\mathrm{X}$ & $\mathrm{X}$ & $\mathrm{X}$ & $\mathrm{X}$ \\
\hline Will is there & $\mathrm{X}$ & $\mathrm{X}$ & $\mathrm{X}$ & $\mathrm{X}$ & $\mathrm{X}$ & $\mathrm{X}$ \\
\hline $\begin{array}{l}\text { Knowledge is } \\
\text { missing }\end{array}$ & $\mathrm{X}$ & $\mathrm{X}$ & $\mathrm{X}$ & $\mathrm{X}$ & $\mathrm{X}$ & $\mathrm{X}$ & $\mathrm{X}$ \\
\hline $\begin{array}{l}\text { Concept of } \\
\text { health.... }\end{array}$ & $\mathrm{X}$ & $\mathrm{X}$ & $\mathrm{X}$ & $\mathrm{X}$ & $\mathrm{X}$ & $\mathrm{X}$ \\
\hline
\end{tabular}

The check shows that five themes (see gray marked themes in the table above) remained because they have a link to the SoC criteria and promote comprehensibility and/or meaningfulness and/or manageability of employees. In addition, the selected five themes show relevance to at least one of the research questions and three themes can be connected to the study by Bruch et al. (2013). The other themes are not followed up by the study. The themes 'Proximity of the boss' and 'Good contact with the boss' are summarised to one theme: 'closeness and good contact with the boss'. Also, the themes 'Boss' authenticity' and 'Trust in the boss' are summarised to the following topic 'trusting and authentic behaviour of the boss'. Summarised, the following themes emerge from the data interpretation of the reflection focus group and are added to 8 findings (represented in chapter 4.2.1) themes:

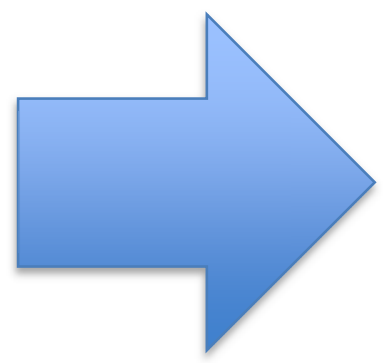

Closeness and good contact with the boss

Trusting and authentic behaviour of the boss

Longing for good old days

4.3.2 Summary and presentation of themes of the reflection group with their contribution to SoC and the factor of the study by Bruch et al. (2013)

This study is based on focus-group discussions and their data interpretation by me as an independent researcher and on a reflection group of three SMEs experts. 
With the reflection group, which does consist of two top managers of SMEs and one employee of SME, they may discover and/or interpret other themes in the data as I may do. I am aware that the interpretations of the reflection group reflect subjective opinions and assessments. Nevertheless, the reflection group can provide helpful supplements to my interpreted themes that should be considered in future research.

The topic 'Closeness and good contact with the boss' regarding the sixth slide (see appendix 9.11) is regarded as relevant in the interpretation of data by the reflection group. This topic is considered by all members. One group member determined: "So, I think for most here, you just need a direct contact with the boss to change something." The topic 'Proximity of the boss' is also found important by the reflection group. A member noted that the focus-group participants presented the topic as important and has noticed that you must have that closeness to the top manager first. That could be a problem if the companies are big and the top manager could therefore not be as present as wished.

Theme contribution to SoC

\begin{abstract}
The theme 'Closeness and good contact with the boss' could strengthen the SoC factors 'comprehensibility' and 'meaningfulness' of employees.
\end{abstract}

The theme 'Closeness and good contact with the boss' does not meet the recommendations of the study by Bruch et al. (2013).

The topic 'Trusting and authentic behaviour of the boss' is found important in the analysis of the statements of employees of the first slide (see appendix 9.11) by the reflection group. It is found that in the statements of the employees the credibility of the top manager plays an important role. His behaviour should match what he communicates. One group member noted: "[...] it would not be credible if he suddenly preached to the staff, "Go to the gym or whatever," because that's not what you know about him, [...] this mentality [...]." The topic 'Trust the boss' is 
regarded as relevant in the analysis of the statements of employees of the fourth slide. The reflection focus group agreed that the basic requirement of a 'meaningful work' is to trust the boss. One member noted: "[...] there is a relationship of trust with his immediate boss, and that is actually the most important for me". boss' could strengthen the SoC factor 'meaningfulness' of employees.

Theme contribution to Bruch et al.
The theme 'Trusting and authentic behaviour of the boss' does not meet the recommendations of the study by Bruch et al. (2013).

The theme 'Longing for good old days' is discussed in the analysis of the statements of the top managers by the reflection group. It is discovered by the group that in the statements of the top managers could be seen that they crave for former times. The interpretation of the reflection group that top managers could crave for the good old times cannot be checked with this study. There could be other or another reason behind the statements of the top managers. Future research would be recommended. One member noticed in this regard: "So there you can see what uncertainty is there and what fears were there, that you may not be able to satisfy." Another member added that there is still an antiquated understanding of leadership in the part of the group like: "earlier the boss was still the patriarch", "I am the top manager, I lead that, and here's how I imagine that, I dictate rules". 
The theme 'Longing for good old days' could strengthen the SoC factor 'meaningfulness' of employees.

Theme contribution to Bruch et al.
The theme 'Longing for good old days' does not meet the recommendations of the study by Bruch et al. (2013).

\subsection{Study findings which answer the study research questions}

The following subchapters present a detailed view of the themes that answer the study research questions and provide a general understanding. It also shows that the themes used to answer the research questions of the study fully promote the SoC of employees because the three SoC criteria are considered sufficiently (the themes fulfil at least one SoC criterion, but mostly 2 to 3 criterions) by the final themes (see chapter 4.2.1 and 4.3.2).

\subsubsection{Research Question 1:}

'What salutogenic factors can be generated by top management to support the promotion of health and well-being of the workforce?' This answer can be given with the help of the themes that are considered important or that are discussed intensively by the focus-group members. As an addition, the themes found by the reflection group are also used to answer the first research questions. I selected nine themes of the total 11 which are suitable as WHP initiatives and also can be implemented by top management: Boss as pioneer and initiator, appreciation by praise, break, Possibility to learn about other areas and to place the own work into the bigger context, corporate culture, trusting and authentic behaviour of the boss, closeness and good contact with the boss, measure more individual and strengthening the sense of togetherness. 
The theme 'Boss as pioneer and initiator' can be generated by top management to support the promotion of health and well-being of workforces. The focus-group members (top management and employees) nonetheless see the responsibility of WHP primarily with the employees. The theme 'Appreciation by praise' is very important according to the statements of the employees of the focus group. Everyone agreed that the appreciation coming from the top manager is important and one of the employees did emphasise the importance of this measure again with the following assessment: "I still think appreciation is one of the most important points". The third theme 'Break' stated that the designing of breaks is an important issue for employees. How employees take a break does not matter to the top managers, they would create the base conditions for it, but they did not want extended break time. The following theme which supports the promotion of workforce health is called 'Possibility of employees to learn about other areas and to place their own work into the bigger context' as another important salutogenic measure, presents the importance of the meaning in employees work. It is important that top managers tell their employees what they contribute to the big picture. 'Corporate Culture' is always strongly shaped by the top manager, his personality and by top managerial family, which makes up the SMEs. The salutogenic measure 'Trusting and authentic behaviour of the boss' could also promote the workforce health and well-being. It is found that in the statements of the employees that the credibility of the top manager plays an important role. His behaviour should match what he communicates. 'Trusting behaviour of the boss' is the basic for understanding the meaning of the workforces according to the reflection group. The factor 'Closeness and good contact with the boss' is also an important theme according to the reflection group. That could be a problem if the companies are big and the top manager could therefore not be as visible or present as desired by the employees. Having a good contact with the boss is an important salutogenic factor in SMEs. The theme 'Strengthen the sense of togetherness' can promote the health and well-being of employees in SMEs. By 
this factor, the top manager and employees get to know each other better and can develop understanding for each other.

In the next step the presented salutogenic factors are stated shortly and precisely so that they can be used directly as WHP initiatives for SMEs in EWL/Germany. I used exploratory analysis to answer the first research question. The approach is based on classic methodology driven by an inductive approach.

\section{Interpretation out of the analysis answering the RQ 1 - the salutogenic factors:}

The top manager should...

- be a pioneer and initiator regarding health promotion and be authentic and be trusting.

- create the framework for breaks.

- $\quad$ enable employees to get to know other company areas \& enable them to place their own work into the bigger context.

- ensure that employees live the spirit and purpose of the top manager family.

- regularly praise their employees and express their appreciation.

- $\quad$ enable the employees to get a good contact with them.

- $\quad$ strengthen the sense of togetherness by offering joint activities outside the work context.

- $\quad$ know the needs of their employees to create individual WHP measures which meet employees' needs.

\subsubsection{Research Question 2:}

'How can SMEs develop top management awareness to keep their staff healthy?' This answer can be given with the help of the themes that are considered important or that are discussed intensively by the focus-group members. As an addition, the themes found (from the statements of the focus groups) by the reflection group are also used to answer the second research questions. I selected three themes of the total 11 which are usable (regarding SoC criterion 'meaningfulness') to promote the top management awareness regarding workforce health and well-being. In summary three salutogenic factors are found and are used to answer the second research question: 'Measures more individual', 'self-responsibility of employees' and 'longing for good old days'. 
The theme 'Measures more individual' should be geared towards employee needs and towards team and company needs. 'Measure should be individual' in order to be effective and therefore successful. Therefore, the regular collection of the needs is necessary to be able to develop suitable WHP initiatives for SMEs. The theme 'Self-Responsibility of employees', made clear that employees feel primarily responsible for their own health and in addition might accept offers from the employer. The theme 'Longing for good old days' is discovered by the reflection group. They have noticed that in the statements of the top managers could be seen that some top managers crave for good old days. It could be a desire for the 'old patriarch that leads his company'.

As above, the three presented salutogenic factors to answer the second question are formulated in a short and precise way so that they can be used directly as WHP initiatives for SMEs in EWL/Germany.

\section{Interpretation out of the analysis answering the RQ 2 - factors to develop top}

\section{management awareness:}

SMEs should provide knowledge for the top management...

- about the importance of their behaviour and attitude in relation to the health and wellbeing of their employees.

- to gain understanding of the importance of promotion of their own health and well-being in relation to employees' health and well-being.

- about the importance of reflecting own aspirations, attitudes and needs (e.g. from generational view) as an older top manager and reflect them in the leadership role.

\subsubsection{Research Question 3:}

'How can SMEs in East-Westphalia Lippe/Germany integrate salutogenic factors to promote the health and well-being of their workforces? The answer can be given with the help of the themes that are already presented in this chapter. The following salutogenic factors found to answer the last research question should present directly in the following diagram: 


\section{Interpretation out of the analysis answering the RQ 3 - how to integrate factors:}

By ...

- strengthening the sense of togetherness by offering joint activities outside the work context.

- motivating the top managers to be pioneers and initiators regarding health promotion and to be authentic and be trusting.

- motivating the top managers to create the framework for breaks.

- enabling employees to get to know other areas of the company and enabling them to place their own work into the bigger context.

- regularly praising their employees and expressing their appreciation.

- enabling the employees to get a good contact with the top management.

- ensure that employees live the spirit and purpose of the top manager family.

- communicating that employees are primarily responsible for their health.

- offering WHP measures that meet the needs of employees and company.

- encouraging older top managers to reflect on their aspiration, attitudes and needs (e.g. from a generational view) and reflect them in the leadership role.

\subsection{Summary of study findings}

This study is based on focus-group discussions conducted by me as an independent researcher in December 2016. Some of the views expressed are personal opinions from those involved and are not necessarily representative of all employers and employees. Nevertheless, it is important to understand perceptions which can provide insights into behaviour.

This chapter gives an overview of the two focus-group discussions of top managers and employees and the reflection-group discussion of family/friends of mine which are also top managers or employees in SMEs. As mentioned previously, I accept twelve requests for group discussion are accepted and 11 are conducted. All the chosen top managers have long-term experience in leading businesses of SMEs. They are open minded generally and interested in health promoting factors for SMEs and currently have no integrated health management system. The second group consists of six employees of the participating SMEs. The type of group members included in this research is described in table 5.

I conducted the ATA based on 11 theme analysis, for example about the role of a top manager 'Boss as a pioneer and initiator' or the behaviour of top manager 
'Appreciation by praising'. The eight themes gathered by my interpretation of the statements top managers and their employees are combined with three themes discussed and interpreted by the reflection group members to answer the three study research questions:

RQ 1 - The following salutogenic factors can be generated to promote the workforce health in SMEs in EWL/Germany: Top managers should be pioneers and initiators regarding health promotion, be authentic and be trusting; should clarify that the main responsibility lies with the employees; should create the framework for a break, should enable employees to get to know other company areas and enable employees to place their own work into the bigger context, should regularly praise their employees and express their appreciation, should enable the employees to get a good contact with them, should ensure that employees live the spirit and purpose of the manager family, should know the needs of their employees to create individual WHP initiatives which meet employees' needs, should enable the employees to get a good contact with them and should strengthen the sense of togetherness by offering joint activities outside the work context.

RQ 2-The following initiatives can be used by SMEs in EWL/Germany to develop top management awareness to keep their staff healthy: The SMEs should provide knowledge for the top management about the importance of their behaviour and attitude in relation to the health and well-being of their employees, should provide knowledge for the top management to gain understanding of the importance of promotion of their own health and well-being in relation to employees' health and well-being and should provide knowledge for the older top management about the importance of reflecting own aspirations, attitudes and needs as a top manager and reflect them in their leadership role.

RQ 3 - The following initiatives can integrate health promoting and well-being factors in EWL/Germany: By strengthening the sense of togetherness by offering joint activities outside the work context, by motivating the top managers to be 
pioneers and initiators regarding WHP and to be authentic and be trusting, by motivating the top managers to create the framework for breaks, by enabling employees to get to know other areas of the company and enabling them to place their own work into the bigger context, by regularly praising their employees and expressing their appreciation, by enabling the employees to get a good contact with the top management, by ensuring that employees live the spirit and purpose of the top manager family, by communicating that employees are primarily responsible for their health, by offering WHP initiatives that meet the needs of employees, by encouraging older top managers to reflect on their aspiration, attitudes and needs (e.g. from a generational view) and reflect on themselves in their leadership role.

The 11 main findings from the study indicate that the quality of the relationships within SME play a major role when it comes to salutogenic WHP. It appears that it is not about the frequency of the contact with the top manager, but about having a good relationship with him/her (e.g. knowing and trusting him). The importance of relationship quality is also evident among the employees in SMEs (strengthen the sense of togetherness by offering joint activities). The finding 'longing for good old days' is a surprising theme which I did not expect. The following chapters discuss the surprising theme in more detail and discuss 'relationship quality' as a contribution of my study for future salutogenic WHP in SMEs in EWL/Germany. 


\section{Discussion of study findings in the context of review findings and}

\section{study research questions}

This chapter discusses the study findings in relation to the study research questions and the literature review findings. I continue to relate the study findings to the SoC theory of Antonovsky and to the nine recommendations of the study by Bruch et al. (2013). It is the opportunity to highlight my research findings which differs from and extends current knowledge of the area in which I have chosen to carry out research. I demonstrate what I found about WHP in terms of salutogenesis in SMEs by interpreting my findings and outlining what relevance the findings may have on employees' health and well-being promotion in a working context. At the end of the chapter I discuss all the findings that I found from my research study and provide and an explanation and interpretation of the research findings in relation to SMEs.

Firstly, I discuss the study findings and their relevance related to salutogenesis. Secondly, I present my research findings (marked with blue colour in the text) and the findings from the literature review (marked with green colour in the text) in a short summary (see table 22). I consider how my findings extend knowledge about the WHP in terms of salutogenesis to connect to the next chapter.

On the basis of two focus-group discussions of top managers and their employees from three SMEs in EWL/Germany 11 themes are considered important and are analysed in order to give important information on how SMEs in this special German region can promote the health and well-being of their employees in the sense of salutogenesis. The research project is based on the assumption that SMEs in Germany do not invest enough in the health and well-being of their employees. As a consultant in SMEs, I realised that SMEs in WHP are lagging behind big companies. The study findings confirm my hypothesis at the beginning of my thesis that it is particularly important that WHP initiatives take a special look at the well-being of employees. It appears that my approach to utilise salutogenesis 
as the theoretical framework is justified. The salutogenesis has another advantage by relating it to the study findings because it allows top managers to understand how health develops and what employees need to stay healthy at work. In other words, I hope through enhancing top manager's knowledge that health and wellbeing of employees are given more importance in SMEs in the future. I also recommend long-term studies to investigate this. Current WHP research also emphasises advantages of salutogenesis in WHP as my literature review shows (see chapter 3 ). It seems to be the most important model in current WHP, as new models in WHP (see Work-SoC by Vogt et al. (2013) or jobs-demands-resources model (JD-R) by Vogt et al. (2016)) build on or link to salutogenesis. Nilsson et al. (2012) note to explore, understand, and foster workplace resources through assistance from the SoC theory. Bauer et al. (2007) integrated salutogenesis as a base in questionnaires which shows benefit in OHM practice by concentrating on salutogenic factors. The advantage of using salutogenesis in WHP is also emphasised by Feldt et al. (2000) by recommending that well-being in the workplace can be increased if there is an understanding of the ways in which work characteristics shape individuals' SoC, and thus their health and well-being at work. Bringsén et al. (2012) and Vogt et al. (2016) use salutogenesis to investigate the job requirements and/or resources of employees, or both, to align them with one another. It seems that current research uses salutogenesis because it can display the needs of employees in the work context more clearly or in more detail. I have noticed that salutogenesis is differently involved in research as already mentioned. Feldt et al. (2000) explore the SoC of employees in connection with employee well-being and Bringsén et al. (2012) bring together the study findings with the SoC criteria. By linking WHP initiatives with the SoC criteria in the study by Bringsén et al. (2012), it was analysed which SoC criteria (meaningfulness, manageability and comprehensibility) are considered by means of WHP initiatives. With this type of involvement of salutogenesis it shows similarities to my study design, because I also had associated the study findings with the SoC criteria (see 
chapter 4). As well as Bringsén et al. (2012) I have expressed the importance of promoting the $\mathrm{SoC}$ in the working context with my study.

The similarity of current research and my study is reflected in the salutogenic intention to research, analyse and present employees' needs and resources in the work context with the help of the SoC. My study confirms that salutogenesis in WHP shows potential to promote employees' health and well-being, and thus confirm the review finding 'Using the SoC theory in WHP' as important. Nevertheless, the results of the review studies are based mainly on employee opinions as I have already mentioned in previous chapters. In order to be able to integrate WHP into terms of salutogenesis in SMEs, the understanding and the willingness of top management are needed first and foremost. For this reason, for a successful implementation in SMEs, it is essential to include the ideas, opinions, reservations or fears of decision-makers and to take these into account. As my data takes the employee side and the top manager side into account my data can supplement the previous WHP research results. There is currently little salutogenic WHP research focusing on SMEs. For this reason, my findings contribute to increasing knowledge in WHP research in SMEs in terms of salutogenesis.

Before I discuss the study findings in connection to the review findings, the study research questions, and the factors of the study by Bruch et al. (2013) I present the 11 study findings and the six review findings in the following table:

Table 22: Overview of study and review findings.

\begin{tabular}{|l|l|}
\hline Study findings & Review findings \\
\hline Boss as pioneer and initiator & Recovery in the workplace \\
\hline Appreciation by praise & Resourceful work environment \\
\hline Break & Positive work experiences \\
\hline Possibility to learn about other areas and to place the & Strengthening of competency \\
\hline Self-responsibility of employees & \\
\hline Corporate culture & Using the SoC theory in WHP \\
\hline Strengthening the sense of togetherness & Leadership \\
\hline
\end{tabular}




\begin{tabular}{|l|l|}
\hline Measures more individual & \\
\hline Closeness and good contact with the boss & \\
\hline Trusting and authentic behaviour of the boss & \\
\hline Longing for good old days & \\
\hline
\end{tabular}

The role as a pioneer and initiator of top managers of SMEs plays a relevant role in WHP as my study has explored. My study confirms the recommendation of the study by Bruch et al. (2013) (see table 1, 'Top management as pioneers of a healthy performance culture') for WHP in SMEs in EWL/Germany (see also chapter 4.2.1). Vinje et al. (2013) emphasise also the importance of the top manager role by stating that leaders must lead WHP processes by scrutinising, discovering and understanding, to improve their own practice as a leader. My data from the focus group of employees shows how important it is for employees that the top manager demonstrates healthy behaviour, and thus confirms to his employees that it is important to look after their own health at the workplaces as the following statement of an employee shows: "So the top manager as a pioneer, clear, [...] maybe even one with the most important points." The top manager should also offer and promote WHP initiatives, as my findings show. Also, Saksvik et al. (2015) confirm that top manager should be initiators of the intervention programme but also should be guides who lead the employees through the intervention process. This factor confirms the current research field of WHP with the review finding 'Leadership' and, on the other hand, contributes to the expansion of WHP knowledge regarding integration of WHP in SMEs. Because my study does not only identify themes as relevant regarding health and well-being promotion of employees, but also gives suggestions on how it can be integrated into SMEs. Therefore, the study finding 'Boss as pioneer and initiator' helps to answer the first and third research questions of my study and could strengthen the SoC factors 'meaningfulness', 'comprehensible' and 'manageability' of employees. As mentioned before, this finding has to be seen in the context of small businesses where the top manager works close with his employees and 
communicate directly with them. In bigger companies all managers should be pioneers and initiators regarding WHP. Nonetheless, it is important that the top manager is the first to take over so that the other managers can follow him. So, this finding relates to a specific form and size of organisations.

My study finds that the 'Appreciation by praise' is important regarding health and well-being promotion in SMEs. The employees of the focus group emphasise that the praise from the top manager is one of the most important factors regarding health and well-being of employees in the working context. One employee in the focus group emphasised the importance with the following statement: "I still think appreciation is one of the most important points." But top managers largely underestimate the importance of praise and have expressed their insecurity regarding praising as the focus group discussion of top manager shows. Looking at this theme in current salutogenic WHP research it is obviously not exposed as a factor that related to employees' health and well-being. But this theme is recommended as an important factor in the study by Bruch et al. (2013) (see table 1, 'Value') and therefore presented in the focus groups. This theme of the study Bruch et al. (2013) is confirmed by my research an important theme regarding WHP in SMEs in EWL/Germany. It could complement existing knowledge in salutogenic WHP research because of deeper information of the focus group participants on how to implement this factor in SMEs (e.g. short praising via feedback after employee successes). This theme is used to answer the first and second research question of my study. The finding 'Appreciation by praise' could strengthen the SoC factor 'meaningfulness' of employees.

Promotion of health and well-being of employees seems to be connected with the opportunity to recover within the workplace as current WHP research shows (Ejlertsson et al., 2018, Nilsson et al., 2012). Ejlertsson et al. (2018) define recovery as a process of psychophysiological unwinding after effort expenditure. Recovery of employees outside the workplace has been studied previously, but since recovery during work is shown to be of great importance in relation to higher self- 
rated health. This factor shows similarity to the study finding 'Break' which shows the employees' wish that the top manager has to design rooms in such a way that employees can rest or occupy themselves e.g. with table football soccer according to their needs. Hence, my study confirms the topic 'recovering' and gives additional suggestions on how SMEs can implement this. The theme 'Break' is used to answer not only the first research question but also the second research question of my study. Moreover, the finding 'Break' could strengthen the SoC factor 'meaningfulness' of employees

The study theme 'Possibility to learn about other areas and to place the own work into the bigger context' is based on the recommendation of the study by Bruch et al. (2013) (see table 1, 'Meaningful work') and is rated by my study as an important theme regarding health and well-being promotion in SMEs in EWL/Germany. The assessments of focus group top managers and employees have shown that it is important for employees to recognise a connection between their work assignment and the success of the company. Recognising the importance of employees' work contributes to their health and well-being. By giving employees the opportunity to get to know other areas of the company the employees' capacity could be strengthened and employees could achieve an understanding of other company areas (e.g. production area) which have a connection with their own workspace. This theme agrees with the following three findings of the literature review: 'Strengthening of competency', 'Resourceful work environment' and 'Positive work experiences' which have a lot in common. All three review findings aim to create optimal conditions for employees in the company so that they can strengthen their skills and can make positive experiences. These literature review findings are also important for salutogenic theory in the workplace because it shows how employees' SoC can be influenced and developed by working conditions (Vogt et al., 2016). Discussion about WHP, so the synthesis recommends, often takes place in correspondence with resourceorientation and strengthen-orientation of workplaces. Vinje et al. (2013) agree and emphasise the importance of co-designing for employees in the working context 
and suggest that employees should are active masters of the work situation. As well as Nilsson (2010) who emphasises that employees should act as creators of upcoming situations. The findings from my research confirm Nilsson's findings about the possibility for employees to act like creators of upcoming situation. SMEs should involve employees in company processes by giving them the capacity to understand the context in which they are working and give also them e.g. the capacity to realise their contribution to the company success, as the literature review findings and my study findings indicate. So, the positive and no longer the negative side of WHP is researched which corresponds to the salutogenic approach of my study and corresponds to the field of 'Positive Psychology'. Consequently, more attention should be centred on developing and increasing positive psychosocial experiences while simultaneously indirectly reducing the negative (Saksvik et al., 2015). This theme contributes to answer the first and third research question of my thesis. In addition, the finding 'Possibility to learn about other areas and to place the own work into the bigger context' could strengthen the SoC factors and 'meaningfulness' of employees.

Recent studies on salutogenic WHP research show that 'Self-responsibility of employees' is an important issue in terms of health and well-being in working context. This theme is closely linked to the empowerment or capacity of employees (discussed in the previous section 'Strengthening of competency') so that employees recognise their own responsibility. Saksvik et al. (2015) emphasise that self-responsibility of employees by increasing employees' ability to take responsibility for their everyday work and their working environment. The top managers in my study have emphasised the employees' personal responsibility as an important prerequisite for their WHP initiatives to also be of interest. However, SMEs need to ensure that employees can become self-responsible for their health at work, as recent studies have shown (Nilsson, 2010, Saksvik et al. (2015). On the other hand, Bruch et al. (2013) notice every employee is responsible for his own health and must not be relieved of this responsibility in the sense of "I'm waiting 
to see what my management offers me" (see table 1 'Health consumption'). The theme is an important one when it comes to health and well-being promotion at the workplace, as my study and current research shows, but care should be taken to ensure that employees could take self-responsibility in this regard. SMEs should not assume that employees have their own responsibility in this regard. This theme contributes to answer the third research question of my study and could strengthen the SoC factors 'comprehensibility', 'manageability' and 'meaningfulness' of employees.

The study finding 'Corporate culture' in terms of WHP is important for SMEs, since WHP initiatives only make sense if they are embedded in a culture enterprise concept which meets the expectations of employees and top management as the following statement of a top manager demonstrates: "We also have to take the people with us, and that's one of the issues of health. And if you then put it into such an instrument and there is a role in health, then I think you have the routine, where that can be a kind of component of the corporate culture at the end". For SMEs in EWL/Germany it is important regarding health and well-being promotion that employees live the spirit and purpose of the top manager. Thus, WHP initiatives that do not fit the corporate culture promise no success in SMEs in $\mathrm{EWL} /$ Germany, as my study shows. This is in line with the recommendation of the study by Bruch et al. (2013) (see table 1, 'Holistic health management'). The various initiatives, which are brought together in companies are more effective when they are interlinked and tailored specifically to the needs of the company and its employees (Bruch et al., 2013). Recent studies do not explicitly discuss this aspect, which might suggest that this is a specific topic in SMEs. This theme contributes to answer the first and third research question of my study and could strengthen the SoC factors 'comprehensibility', 'manageability' and 'meaningfulness' of employees.

Findings from my research study show that the theme 'Strengthening the sense of togetherness' is relevant to health and well-being promotion in SMEs. The top managers of the focus group have made good experiences in meeting outside the 
working hours with their employees. One top manager stated: "[...] what we have noticed is that we have a better atmosphere in the company if we do something together on a regular basis". This theme shows that events are important where employees and managers can exchange views and gain positive experiences as a team outside of work. Strengthening employees' sense of belonging seems to be an important aspect in health and well-being promotion of employees in SMEs. Perhaps the theme of togetherness is typical of SMEs, as this company size is often working under the motto 'Together we can do it' as this statement of a top manager showed: "[...] I say to all employees, that's all of us, that is. So, everyone is a building block [...]". The sense of togetherness is an important prerequisite for employees in SMEs in EWL/Germany to feel healthy and comfortable. Only the study of Saksvik et al. (2015) confirm the study finding and found that it is important to come together outside of the daily work to get to know each other better. It would be of interest if the study by Saksvik et al. (2015) gathers their data also in SMEs. This finding has no connection to the topics of the study by Bruch et al. (2013) and can therefore complement the guideline of Bruch's study. My research finding 'Strengthening the sense of togetherness' is used to answer the first and third research questions. Further, it could strengthen the SoC factors 'comprehensibility', 'manageability' and 'meaningfulness'.

The study finding 'Measures more individual' has been widely discussed in current WHP research (see review findings 'Resourceful work environment', 'Positive work experiences', 'Strengthening of competency') by e.g. Bringsén et al. (2012), Vinje et al. (2013) or Ejlertsson et al. (2016). For example, Feldt et al. (2000) suggest promoting employees' well-being focussing on their individual SoC. Both, in my study and in current research, it is recommended that WHP initiatives should not only be appropriate for the individual but also for the team (e.g. Vinje et al. 2013). My study goes a step further and has determined that WHP initiatives should also suit the company. For this purpose, it is necessary for SMEs in EWL/Germany to review the employees' needs at regular intervals in order to be able to develop suitable WHP initiatives which should also meet the company character. By 
involving the top management, the WHP initiatives can be designed to suit the corporate culture in the company. On the one hand, the theme 'Measures more individual' confirms current WHP research and adds important information on alignment with corporate culture of companies. This insight is confirmed by the study of Bruch et al. (2013) with the factor 'Holistic health management' which is similar in the intention to focus on individual and company needs regarding WHP initiatives. The finding 'Measures more individual' contribute to answer my third research question and further, could strengthen the SoC factors 'comprehensibility', 'manageability' and 'meaningfulness' of employees.

A further important finding of this study is the theme 'Closeness and good contact with the boss'. The quality of the relationship with the top manager seems to be an important factor in SMEs in EWL/Germany and is not found in current WHP research. Since this topic in this form is not discussed in current WHP research, I assume that it is related to the type of SMBs. Especially in SMEs, it goes without saying that you know the top manager and even work directly with him. The need to have good contact with the top manager may therefore be higher in SMEs than in big companies. The quality of relationship to the top manager could be an important factor in broadening current WHP research in SMEs. Top managers should therefore look for a good relationship with their employees. The important topic of relationship quality in my study is underpinned by another of my study's finding, named 'Trusting and authentic behaviour of the boss'. It is found that in the statements of the focus group participants the credibility of the top manager plays an important role. His behaviour should match what he communicates. Top managers in SMEs should only introduce WHP initiatives that they can represent themselves and which they consider to be important. When I compare the current WHP research with my findings of 'Closeness and good contact with the boss' and 'Trusting and authentic behaviour of the boss', I notice that the relationship quality from the top manager to employees in this form is not found. Current WHP research shows, that the leadership plays an important role for the health and well-being of employees (e.g. Feldt et al. 2000, Bruch et al. (2013), Vinje et al. 
2013). In this context, the role of top manager as an initiator and pioneer of WHP initiatives takes place in current research but the importance of trust towards top managers, authenticity of top managers and good and direct contact of employees with top managers could not be found. No recommendation of the study by Bruch et al. (2013) regarding 'Closeness and good contact with the boss' and 'Trusting and authentic behaviour of the boss' can be found either. Therefore, my study can contribute to expand existing knowledge in the field of WHP research in order to promote the health and well-being of employees in SMEs. Further, this finding contributes to answer my first and second research question. Moreover, the finding 'Closeness and good contact with the boss' could strengthen the SoC factors 'comprehensibility' and 'meaningfulness' of employees. The finding 'Trusting and authentic behaviour of the boss' could strengthen the SoC factor 'meaningfulness' of employees.

The finding 'Longing for good old days' 'is a further finding of my study. The top managers repeatedly made comparisons to earlier times (post- war periods etc.) at various points in the focus-group discussion (see chapter 4.3.2). The interpretation of the reflection group that top managers could crave for the good old times cannot be checked with this study. Of course, there could be other or another reason behind the statements of the top managers. Further research would be recommended. However, current WHP research considered older workers and their needs regarding WHP as my literature review shows (e.g. Vinje et al., 2013) but top managers are not analysed so far. The needs of the employees are therefore in the foreground of current WHP research. Nevertheless, the needs of employees and top managers could be analysed through the theory of generation types. Generations can be influenced by generational experiences, formative experiences in childhood or adolescence, which have an influence on the whole birth year. Examples of this are wars or post-war periods, which have strongly influenced the population in their daily activities (Wiedmer, 2015). From the birth year 1922 until now five different generations are divided according to the period of their birth: 
- Traditionalists (1922-1945)

- Baby boomer (1946-1964)

- Generation X (1965-1983)

- Generation Y (1984-2002)

- Generation Z (from 2003)

Assigning generations to models as an analytic category is becoming more and more critical as Boatin (2008) noticed. But at the same time parts of the research still advocate Karl Mannheim's conviction that 'real social and spiritual contents' create a 'real connection' between individuals, which can be seen as a 'generational context' or as 'generational units' and have them examined (Mannheim, 1970). I choose the generational type model because it is very descriptive and summarises important information into generations, even if it is not comprehensive and accurate.

The traditionalists, born between 1922 and 1945, witnessed the end of the 'First World War' and 'World War II' in their childhood and youth. The baby boomers, born between 1945 and 1965, were the first post-war generation after 'World War II', which have experienced the 'economic miracle' (called 'Wirtschaftswunder' in Germany) in post war Germany and belong to the birth-richest years. My study finding 'longing for good old days' is based on the study participants (focus group top manager and reflection group) which are even older than 55 . Therefore, the generation of 'traditionalists' (1922-1945)' or 'baby boomer' (1945-1965) should be explored more closely in further research. Furthermore, SMEs have problems to find successors, so top manager are leading their companies into old age. In 2016, 39\% of all business owners of SMEs are 55 years or older (Schwartz et al., 2018). The upcoming generational change in German SMEs is increasingly becoming a problem (Schwartz et al., 2018). Because of this condition it is important to take a closer look at the generation types of the top manager and his values or needs, for example, to choose WHP initiatives to suite the top managerial point of view. It is also important to consider the employees' needs 
through generation types to have the possibility to compare the needs and values of both. I named the finding 'longing for good old days' to 'encouraging older top managers to reflect on their aspiration, attitudes and needs' (e.g. from generational view) and reflect them in their leadership role because it represents a WHP initiative, which not only aims to the feeling of longing for old times. The considerations of the top manager generation type and their special needs is an important insight of this study and have implications of further research, which will be discussed further in the next chapter. This theme is not discussed by the study of Bruch et al. (2013) and could therefore expand the guideline of the study by Bruch. My study finding contributes to answer the second and third research question. In addition, the finding 'encouraging older top managers to reflect on their aspiration, attitudes and needs' could strengthen the SoC factor 'meaningfulness' of employees.

The factors of the study by Bruch et al. (2013) 'Healthy self-management', 'Freedom with a safety net', 'Challenging and supporting' and 'Psychological immune system' (see table1) could not be confirmed by my study. In other words, the focus-group participants did not at all, or intensively, discuss these topics and did not consider them important. So, when it comes to implementing the nine recommendations of the study by Bruch et al. (2013) in SMEs in EWL/Germany, my study gives relevant information which WHP initiatives are suitable and which tend to be less significant for SMEs.

My findings show potential to strengthen the SoC of employees as mentioned in chapter 4. Viewed in a differentiated way, all findings from my research study fulfil the SoC criterion 'meaningfulness'. Seven findings strengthen the SoC criterion 'comprehensibility' and four study findings strengthen the SoC criterion of 'manageability'. Therefore, my study recommends WHP initiatives which are meaningful for employees and top managers of SMEs in EWL/Germany. The question remains unclear whether it is particularly important for SMEs, especially 
in their WHP to make sure that initiatives meet the SoC criterion 'meaningfulness'. Thus, I recommend exploring this in future research.

Summarised, eight of my study findings confirm the current state of WHP research and provide important information on how WHP initiatives can be integrated into SMEs. My study adds recommendations for salutogenic WHP implementation in SMEs. In addition, three study findings provide also new insights into the meaning of relationship quality in SMEs and the importance of encouraging older top managers to reflect on their aspiration, attitudes and needs (e.g. from a generational view) as mentioned in the previous chapter. The importance of relationship quality focuses on the relationship of top managers with their employees (and vice versa) and among the employees in SMEs. My study finding 'encouraging older top managers to reflect on their aspiration, attitudes and needs' is a surprising theme, which I did not expect.

Summarised, my study provides important information how to integrate salutogenic WHP into SMEs in EWL/Germany as a contribution to practice. My study delivers new findings to current WHP research, namely the importance of the quality of the relationship between top managers and employees, and the importance of the capacity of older top managers to reflect. Both study findings will be discussed in the following chapter as a contribution for future salutogenic WHP practice in SMEs in EWL/Germany and have also as a contribution to new knowledge in theory. 


\section{Implications for further research}

The study about salutogenic factors promoting workforce health and well-being in SMEs in EWL/Germany leaves space for further and more detailed research. The most potential areas for further research are the phenomena 'importance of the quality of the relationship' between top managers and employees, and the 'importance of the capacity of older top managers to reflect'.

Top managers in SMEs in Germany are increasingly leading companies in their old age because SMEs in Germany have difficulties finding successors for years as mentioned before (Schwartz et al., 2018). It is important to consider older top managers regarding WHP because they are the ones who decide whether WHP will be integrated into the company and those who act as role models in matters of health and well-being. WHP in SMEs should address the age of top managers as Bund (2014, pp.7, translated by myself) notices: "Those who are still there are tenacious [...] in positions where they can do a lot". Therefore, it is worth to look at the values and characteristics of this generation." In order to understand the needs of the older generation of the 'traditionalists (1922-1945)' and 'baby boomer' (1945-1965), it is important to look at the circumstances that prevailed in the World War II or post-war period. The 'traditionalists' experienced the privations and horrors of World War II. Their youth was influenced by the post-war emergency situations. Everyone had to help with the reconstruction and to secure their own existence. Then this generation experienced the rapid economic upswing, the so-called economic miracle (Bund, 2014). In a professional context, the traditionalists are characterised as very dutiful and their life motto is 'first the work, then the pleasure'. They are humble, loyal and do not consider themselves 'the centre of the universe' (Bund, 2014).

Following World War II, the average age of marriage dropped, and the number of children increased dramatically, making the 'baby boomer' generation substantially larger than the 'traditionalists' (Kane, 2018). In the early years of the boom, schools were overcrowded, colleges did not have enough seats, and 
competition for starting jobs was intense. The generation of the baby boomer learned to compete for resources and success (Kane, 2018). 'Baby boomers' are confident, independent and self-reliant. This generation grew up in an era of reform and believe they can change the world. They questioned established authority systems and challenged the status quo. In the workplace, "baby boomers' are not afraid of confrontation and will not hesitate to challenge established practices. They are also excellent mentors (Wong et al., 2008). "In general, older workers (both the Traditionalist generation and the Baby Boom generation) are very positive about themselves and the companies they work for. They see themselves as more reliable than younger workers, more productive, and as having great loyalty to the company. Indeed they have the highest employee engagement scores." (James et al., 2007, p. 7).

Even though the analysis of the needs, attitudes etc. of older top managers is carried out according to generational models by my study (even though the generation model is critical, as I mentioned earlier), it nevertheless helps to understand some level of conflict that may exist among top managers of the oldest generation and the young employee generations in today's workplace. An important aspect here is to make the top managers aware of their needs of their generation and to name the values of young generations to which their employees count. The generational models could help to understand why older top managers of my study long for good old times. One assumption is that they no longer fully meet the rising demands of the working world today (e.g. digitization) and therefore long for good old times in which their competencies coincide with the requirements. Further investigation would be needed to substantiate this claim. It is important to consider the background of the older generation of top managers when it comes to implementing WHP in SMEs. Consultants who help SMEs to implement WHP should consider that older top managers should identify with WHP. On the other hand, it is important for salutogenic WHP in SMEs that top managers reflect their needs, attitudes etc. in their trend setter role regarding 
health promotion towards their employees. In other words, older top managers should reflect their values, views and aspirations and not equate them with those of their employees. For further WHP research in SMEs it would be important to investigate, whether strengthening the capacity of older top managers to reflect and the possibility of older top managers to identify with WHP, promote the health and well-being of employees in SMEs.

Furthermore, the study finding 'importance of the quality of the relationship' between top managers and employees has also potential for further research. The gathered salutogenic factors of the study supporting the promotion of health and well-being of workforces in SMEs in EWL/Germany are closely linked to the relationship between top managers and employees. Of course, the leadership style of top managers in SMEs also plays a role here, but not only. The study finding 'importance of the quality of the relationship' goes beyond leadership. It is not the frequency or quantity of the contact with the top manager but the quality of the contact between top manager and employees is of importance, as my study shows. Especially in SMEs top managers play a very important role because they are more approachable unlike in big companies and the top manager is often associated directly with the product. The personal or corporate spirit prevalent in SMEs (as mentioned in chapter 4) may result in employees wanting closer contact with the top manager than in large companies. Employees want to know the top manager well and vice versa. Further investigation would be needed to substantiate whether the health and well-being of employees in SMEs depends on the quality of the relationship between the top managers and their employees. An interesting consideration for future research can be summed up in the following questions: 'Do SMEs need a special WHP system that addresses the generation of older top manager? And 'How can be increased the relationship quality between top managers and employees in SMEs?'

Summarised, the study findings 'relationship' and 'capacity to reflect' could have implications for further WHP research in SMEs as they are more intensively discussed by the participants of the focus groups than other topics. 


\section{Conclusions of salutogenic WHP research in SMEs}

The following conclusion is structured into three main parts. The first part is an introduction to the conclusion chapter and the explanation of why it is necessary to write this thesis. The second part displays the implications and limitations of research findings. It synthesises the research findings of my study according to the three research questions. The conclusion to the answers of the study research questions are based on current knowledge and academic science from the literature review and the results of the research analysis. Further, I display the surprising outcome of my study. A contribution to knowledge can be given based on the implications for theory, research and practice as proposed by Jankowics (2005), Ghauri (2005), Saunders (2011), and Whetten (1989). The third part reflects on the introduction of my thesis, and whether the study contributes effectively to the significant problems outlined in the beginning.

\subsection{Relevance of research}

This research has importance and relevance for SMEs because it provides concrete WHP initiatives suitable for the needs of this special business size based on the theory of salutogenesis (SoC). With the framework of salutogenesis my study takes the physical and mental side of health of employees in SMEs into account. Therewith, my study does justice to the current trend in WHP focussing on the mental side of employees' health. There is a knowledge gap in the field of salutogenic influences in the context of the economic field (Zimber, 2011). For the first time, the SoC model of Antonovsky (1979) is combined with a current study by Bruch et al. (2013) to investigate whether, or to what extent, salutogenesis is applicable in SMEs in EWL/Germany and whether, or to what extent, current WHP can promote the SoC of employees, and thus promote the well-being and health of employees.

I design this study to explore how top managers in SMEs evaluate salutogenic 
WHP initiatives and to understand how useful they are for the top managers. Another goal of my research is to provide an understanding of how SMEs in EWL/Germany integrate salutogenic WHP. This topic in the field of SMEs is completely new and no study has yet been conducted in this form.

To focus the research according to these conceptual aspects my study answers the following research questions:

Table 23: Research questions and some research findings.

\begin{tabular}{|c|c|c|}
\hline & Research questions & Some of the research findings \\
\hline RQ 1 & $\begin{array}{l}\text { What salutogenic factors can be } \\
\text { generated by top management to } \\
\text { support the promotion of health and } \\
\text { well-being of the workforce? }\end{array}$ & $\begin{array}{l}\text { The top manager should... } \\
\text {...be a pioneer and initiator regarding health promotion and be } \\
\text { authentic and be trusting. } \\
\text {...create the framework for breaks. }\end{array}$ \\
\hline RQ 2 & $\begin{array}{l}\text { How can SMEs develop top } \\
\text { management awareness to keep their } \\
\text { staff healthy? }\end{array}$ & $\begin{array}{l}\text { SMEs should provide knowledge for the top management... } \\
\text {...about the importance of their behaviour and attitude in relation to } \\
\text { the health and well-being of their employees. } \\
\text {...to gain understanding of the importance of promotion of their } \\
\text { own health and well-being in relation to employees' health and } \\
\text { well-being. }\end{array}$ \\
\hline RQ 3 & $\begin{array}{l}\text { How can SMEs in East-Westphalia } \\
\text { Lippe/Germany integrate salutogenic } \\
\text { factors to promote the health and } \\
\text { well-being of their workforces? }\end{array}$ & $\begin{array}{l}\text { By ... } \\
\text {...strengthening the sense of togetherness by offering joint activities } \\
\text { outside the work context. } \\
\text {...motivating the top managers to be pioneers and initiators } \\
\text { regarding health promotion and to be authentic and be trusting. }\end{array}$ \\
\hline
\end{tabular}

In conclusion, relevance of my study is briefly summarised in the following points:

First, SMEs are often referred to as the heart of the German economy because they provide most of the economic output, train and employ most employees, and contribute significantly to corporate tax revenue in Germany. Consequently, WHP should become more and more important to SMEs to keep their most valuable asset, the workforce healthy, and enable them to work.

Second, my study is important because it is practice-orientated and explains how SMEs can promote their employees' health and well-being. Very concrete initiatives are presented with my study that can be applied across all sectors in SMBs. 
Thirdly, my study pursues a turnaround of WHP away from the cause of disease research to the cause of what makes employees healthy. The new perspective in WHP is already being considered e.g. in social and health sectors but not in business yet.

Fourth, my study supports SMEs in their search for specialists and executives by offering innovative WHP. Hence, SMEs can compete with large companies that have already discovered WHP as a trend.

Fifth, my study supports future WHP research by highlighting the needs, resources, and concerns etc. of WHP of SMEs.

Sixth, my study supports future WHP research in promoting health, especially mental health or well-being, using the theory of salutogenesis in WHP.

\subsection{Implications and limitations of research findings}

\subsubsection{Qualitative research design}

I included existing research on salutogenic WHP in the field of businesses with the scope of the literature review. I considered scientific and practical perspectives, which serve as a foundation for the design and subsequent analysis of the focusgroup discussions. Regarding the methodological approach for the qualitative text analysis, I choose the ATA (see chapter 2.6.1). That approach focuses on the themes and aspects that emerge from focus-group discussions and reflection group content, which assists in exploring how SMEs can develop top management awareness to keep their staff healthy and in understanding how SMEs integrate salutogenic WHP.

In this study, understanding is generated from two focus-group discussions and one reflection-group discussion. The validity should be understood in terms of the researcher's critical realist paradigm. This view considers that the social world, so also in SMEs, is constantly changing. The critical realist view, as Saunder (2011) noticed, is in line with the purpose of business and management research which is 
often to understand the reasons for phenomena as a precursor to recommending change. A critical realist position in the context of my study considers the aim to explore and develop salutogenic WHP initiatives in SMEs. The analytic purpose has a descriptive and mainly exploratory orientation that is content driven. The research goal is a description of a specific phenomenon supported by an interpretation of meaning in available text. The information gained from the literature review, the analysis of the focus-group discussions and the reflectiongroup discussion, and the research design enables me to answer the three study research questions. Moreover, I choose a qualitative approach, ATA, to gain meaning, understanding, and recommendations from the three study research questions to achieve the selected research aim. In answering the three study research questions the rationale of my thesis (to help SMEs keep employees healthy because they are the most important resource for businesses in the future) becomes clear again.

\subsubsection{Research answer 1: Exploration of salutogenic factors supporting the promotion of health and well-being of workforces.}

\section{What are the salutogenic factors promoting workforce health and well-being generated by top management in East-Westphalia Lippe/Germany?}

This answer can be given with the help of the themes that are considered important by the focus-group members. The themes found by the reflection focus group and the review findings are also used to answer the first research question. I selected the following nine study findings of the total 11 which are suitable as salutogenic WHP initiatives and also can be implemented by top management: 'Boss as pioneer and initiator', 'appreciation by praise', 'break', 'possibility to learn about other areas and to place the own work into the bigger context', 'corporate culture', 'trusting and authentic behaviour of the boss', 'closeness and good contact with the boss', 'measure more individual' and 'strengthening the sense of togetherness'. I will no longer describe the salutogenic factors in detail that I have 
already discussed in chapter 4.4., and chapter 5 . In order to answer the first question, I use the review finding 'leadership', because it compliments my study findings. My study findings have some interfaces on the topic of leadership, especially when it comes to the behaviour of top managers (e.g. appreciation by praise). I would like to use it as a complementary factor to answer the first research question, as it is an important factor in current WHP research to promote the health and well-being of workforces. For answering the first research question in a few words it can be summarised:

The salutogenic factors promoting workforce health and well-being can be mainly found in the quality of relationship of top managers with their employees that are characterised by trust, appreciation, empathy and authenticity.

\subsubsection{Research answer 2: Exploration of how SMEs can develop top management awareness to keep their staff healthy}

\section{How can SMEs develop top management awareness to keep their staff healthy?}

This answer can be given with the help of the themes that are considered important or that are discussed intensively by the focus-group members. As an addition, the themes found by the reflection group are also used to answer the second research questions. In summary three salutogenic factors are found and are used to answer the second research question: 'Measures more individual', 'self-responsibility of employees' and 'encouraging older top managers to reflect on their aspiration, attitudes and needs' (e.g. from generational view). The review findings confirm my study findings 'measures more individual' and 'selfresponsibility of employees' and my study finding 'encouraging older top managers to reflect on their aspiration, attitudes and needs' is not found in current salutogenic WHP as mentioned before. Three salutogenic factors are 
found to answer the second research question. For answering the second research question in a few words it can be summarised:

SMEs in EWL/Germany develop top management awareness to keep their staff healthy by strengthening the capacity of top managers to reflect and informing and sensitising the top managers about the importance of promoting workforce health and wellbeing.

\subsubsection{Research answer 3: Understanding of how SMEs in East-Westphalia} Lippe/Germany integrate health promoting and well-being factors.

\section{How can SMEs in East-Westphalia Lippe/Germany integrate salutogenic factors to promote the health and well-being of their workforces?}

This answer can be given with the help of the themes that are considered important or that are discussed intensively by the focus-group members. As an addition, the themes found by the reflection group are also used to answer the third research questions. The answer can be given with the help of the themes that are already presented in chapter 4.4. All 11 study findings can help SMEs in $\mathrm{EWL} /$ Germany to integrate salutogenic factors: By strengthening the sense of togetherness by offering joint activities outside the work context, by motivating the top managers to be pioneers and initiators regarding WHP and to be authentic and be trusting, by motivating the top managers to create the framework for breaks, by enabling employees to get to know other areas of the company and enabling them to place their own work into the bigger context, by regularly praising their employees and expressing their appreciation, by enabling the employees to get a good contact with the top management, by ensuring that employees live the spirit and purpose of the top manager family, by communicating that employees are primarily responsible for their health, by offering WHP initiatives that meet the needs of employees and by encouraging 
older top managers to reflect on their aspiration, attitudes and needs (e.g. from a generational view) and reflect on them in their leadership role.

The 11 study findings contain four out of five review findings (see chapter 5) and therefore they are not mentioned again in order to answer the third question. I use the review finding 'leadership' in addition to my 11 study findings to answer the third question as it is an important topic in current WHP research as mentioned before. As with the first and second research question, I will no longer describe the factors in detail. For answering the third research question in a few words it can be summarised:

SMEs in EWL/Germany can integrate health promoting and wellbeing factors in which they develop their top management who value a good relationship with their employees and who see themselves as experts in matters of health and well-being promotion. SMEs should know and understand the needs of their employees to create structures that meet the needs of employees.

\subsubsection{Limitations of research study}

The principal findings of my study are based on the personal beliefs of the focus groups and reflection group participants who viewed WHP as a contribution to successful small and medium-sized businesses (SMBs). Because every participant of my study is an individual case, the research can only present a situated truth (Bryman et al., 2007). The basic tenet of qualitative research states that no absolute truth exists.

A further limitation of my study is the result of research based on a specific group of employees and top managers in SMBs and family and friends of mine. My research is based on a small sample size. Even though it is about a few participants in my study, the experiences and testimonies of these participants are valuable, and I respect their different views. Because of the specific focus on German SMEs, 
and their attitude and use of salutogenic WHP initiatives there is a certain limitation regarding the English language. It is more difficult to present a German topic in a foreign language. Moreover, it should be noted that the study has collected its data from a specific region of German that is characterised by wellknown SMEs. Hence, the study findings should be considered in the context of this region and are unlikely to be generalised to other parts of Germany with different business-structures. It needs to be acknowledged that a generalisation of my research study's findings and contribution to practice for SMEs could not be claimed. Nevertheless, my study findings at an abstract level represent salutogenic variables that salutogenesis as a model has researched as relevant to WHP within micro and macro systems. It is thereby applicable generically to organisations and their sub-systems to an extent. In other words, my research findings cannot be globally transferred as such, but the topics may well be trialled in other settings for research and outcome testing.

I plan to collect qualitative data from SMEs from another region of Germany and I want to explore whether the older top managers also discuss about past times in relation to WHP in my future research. With the findings of my study I would pay attention to the distribution of the age within the focus groups and let several older top managers and several younger top managers discuss about salutogenic factors (as well as with the employees) in my future research.

These are the main contributing limitations of the study.

\subsubsection{Surprising outcome of this study}

A surprising outcome of this research is the theme 'encouraging older top managers to reflect on their aspiration, attitudes and needs' (e.g. from generational view) and it is discussed by the focus group of top managers. The reflection group justifies this discussion with the feeling of 'longing', and with the fear of loss after familiarity. The study finding is based on the study participants (focus group of top managers and reflection group) which are older than 55 . 
Whether the higher age of participants explains the 'longing of the past' requires future research to be clarified. The considerations of aspiration, attitudes and needs of older top managers are an important insight of my study.

\subsubsection{Contributions to knowledge}

The contributions to knowledge of my study are based on connections between the theory of salutogenesis, the practical guideline within the study by Bruch et al. (2013), the review findings and my study findings. The connection between the theory of salutogenesis and the study by Bruch, and the link of current salutogenic WHP research with my data on the applicability of salutogenic WHP in EWL/Germany are based on the rationale of my thesis (see chapter 1.8).

My study provides as a contribution to the theory salutogenic factors that have been explored for applicability in SMEs in a special region in Germany. It supplements quantitative WHP research with important insights into which salutogenic factors can be used in SMEs. My study provides an important contribution to knowledge by providing information to understand the phenomenon of health and well-being promotion in SMEs. Ordinal values are not to the fore but rather the context of social issues - the top managers' attitude to WHP resulting in a verbal description of the research findings. In doing so, I am providing extensive quantitative WHP research information from the SME field and this allows future research to get a comprehensive know how in WHP in SMEs. In addition, my study represents two new salutogenic factors that have not yet been found in current WHP research: 'importance of the quality of the relationship between top managers and employees', and the 'importance of the capacity of older top managers to reflect'.

At the centre of the knowledge contributions of my study is the insight on the importance of the key role of the top manager as a confidante that can be important for leadership research or general research in SMEs because it emphasises the outstanding importance of relationship quality in SMEs. Moreover, 
my thesis makes an important contribution to the topic of aging top managers in SMEs in relation to WHP because it provides an important insight which contributes to the understanding of older top managers when it comes to innovations like salutogenic WHP.

Summarised, my study developed a new WHP method with a special focus on mental health that can be geared to the needs of SMEs in EWL/Germany which can be replicated and implemented to other SMEs in EWL/Germany. The contributions of my thesis to knowledge fulfil the purpose of filling the research gap in salutogenic WHP in SMEs that I found through the SLR (see chapter 3).

\subsubsection{Implications for theory, research and practice}

My study has implications for the theory of salutogenesis because it has explored that it can be connected to current WHP models like the guideline within the study by Bruch to promote the health and well-being of employees in SMEs. My study can claim that salutogenesis is applicable in SMEs. In addition, my findings show potential to strengthen the $\mathrm{SoC}$ of employees as mentioned in chapter 4 . Viewed in a differentiated way, all study findings fulfil the SoC criterion 'meaningfulness', seven findings strengthen the SoC criterion 'comprehensibility' and four study findings strengthen the SoC criterion of 'manageability'. Therefore, my study recommends WHP initiatives which are meaningful for employees and top managers of SMEs. For further requirements research needs to concretely apply salutogenic criteria of Antonovsky's theory into SMEs practice. Experiences of practical implementation of the SoC theory in SMEs are necessary. In general, salutogenesis as a basis in WHP could take care that SMEs focus also on mental health promotion not only on the physical side of WHP. I recommend long-term studies in SMEs to confirm my assumption.

Eight out of 11 study findings confirm the current state of WHP research which means that three study findings complement the current WHP research that I named in two phenomena: 'relationship' and 'capacity to reflect'. Both have 
potential for further research as mentioned before (see chapter 6). In general, it is advisable to explore the salutogenic WHP initiatives of my study which are based on my research findings and the findings of the literature review (see chapter 3 ) in order to answer a further question 'Can salutogenic WHP initiatives in SMEs in EWL/Germany promote the health and well-being of employees sustainably?'

The review findings of my study also have a connection to the guideline within the study by Bruch because eight of ten review studies show similarities with the nine factors of the study by Bruch in their themes. My study could confirm that the guideline by Bruch includes current WHP initiatives which have scientific foundation and could also confirm that Bruch's factors are mostly suitable for SMEs (as the review studies also mostly focus on small and medium-sized organisations). Nevertheless, by exploring Bruch's factors for applicability in SMEs in EWL/Germany, my study findings complement and modify the guideline by Bruch to be able to apply it to SMEs. My study provides new insights for future WHP research and supports WHP research by highlighting the needs and resources of SMBs. Moreover, my thesis supplies knowledge for future research in promoting workforce health, especially mental health or well-being, using the theory of salutogenesis in WHP. Thus, my thesis can be regarded as an important starting point for a salutogenic WHP method that can be modified to all businesses-sizes.

My study gives practical recommendations for SMEs in EWL/Germany regarding the health and well-being promotion of their workforces that emerged from the focus-group and reflection group discussions relevant to value-based information.

The gathered salutogenic factors of my study supporting the promotion of health and well-being of workforces are closely linked to the role of the top manager. Of course, the leadership style of the top manager also plays a role here, but not only. The study includes factors that go beyond leadership. The three research questions produced the following findings that contribute to practice: 
1. Representation of salutogenic factors which promote workforce health and well-being generated by top management in EWL/Germany. Since the salutogenic factors promoting workforce health and well-being can be found mainly in the quality of the relationship of top manager to his employees, my study could help SMEs to deal with the quality of top manager-to-employee relationships.

2. Display of concrete measures to develop the awareness of top managers to keep their workforce healthy by strengthening the capacity of top managers to reflect and informing and sensitising the top managers about the importance of promoting workforce health and well-being.

3. Explanation of how SMEs in EWL/Germany can integrate health promoting and well-being factors in which they develop their top management into leaders who value a good relationship with their employees and providing them with support. The study also contributes to practice while giving them initiatives how to know and understand the needs of their employees to create structures that meet the needs of employees.

Because of the small sample-size it must be acknowledge that a generalisation of my study findings and contribution to practice for SMEs could not be claimed. This is a new area of research and my research findings are a contribution to knowledge and understanding of an individual's perception and views about salutogenic WHP. The value of the study lies in the qualitative research approach and listening to the views of employers and employees.

In conclusion, regarding the implications for practice, theory and research, I learned that successful salutogenic WHP in SMEs needs competent top managers who reflect their values, attitudes etc. in their leadership role and maintaining good relationships with their employees characterised by trust, appreciation, empathy and authenticity. 


\subsection{Recommendations for further research and practice}

In addition to this study, an adaptation of in-depth workshops with the SMEs which participated is conceivable to identify, develop, and to test the use of salutogenic WHP initiatives. A long-term study to develop further insights would be beneficial. An additional option could integrate the study of salutogenic factors with a comparison between big companies and SME businesses.

Because a key result of this study is the attitude and role of the top manager to adapt salutogenic WHP to their business, further research about values and standards of top managers in SMEs is appropriate. Also 'change models' could be another layer to discuss in the context of salutogenesis. As already discussed, the aspect of generation types and their specific needs and experiences could be an interesting aspect for future research regarding salutogenic WHP. An interesting consideration for future research in SMEs can be summed up in the following question: Do the SMEs with their older top managers need a specific approach for the need for WHP and a specific explanation and support of the creation of health in the first step?

For SMEs in EWL/Germany I recommend integrate the WHP action plan based on answers of the third research question of my study. I recommend including the health insurance companies. It is also helpful to use networks that provide assistance and opportunities regarding $\mathrm{CHM}$ for sharing with other companies. On the one hand, these may be regional networks, but on the other hand also superregional networks such as the 'Proactive SMEs' (called 'Offensive Mittelstand' in Germany). Top managers of SMEs, business consultants of SMEs and $\mathrm{CHM}$ experts meet here to exchange views on the theme CHM for SMEs. The new consulting and information portal 'www.bgf-koordierungsstelle.de' also bundles the nationwide counselling and support offers for CHM, where SMEs can obtain information. In the first step, business consultants and coaches for SMEs in EWL/Germany could be helpful to integrate my salutogenic WHP action plan. 
Even though my study findings are not generalisable, they may still contain useful WHP initiatives that are relevant to SMEs in general.

\subsection{Final remarks}

In conclusion, my thesis makes clear that salutogenic factors promoting health and well-being of workforces generated by top management in EWL/Germany are characterised by the capacity of top managers to reflect and their attitude and values regarding health and well-being of employees.

My findings are to be considered in the context of SMEs in a specific region of Germany, where there are many well-known companies of this size. So my study can help empower SMEs to meet their challenges by creating business conditions to keep their employees healthy. My thesis considered the resources of SMEs and developed a WHP action plan that is easy to implement and noticed also the cultural change by considering the aging top management in SMEs. Thus, my study does justice to the needs of SMEs.

I have found that SMEs have not really, or only occasionally, taken care of the health and well-being promotion of employees. Background knowledge about WHP and the knowledge of necessity of health and well-being promotion are lacking in many places. My study also shows that SMEs are prepared to take a closer look at the topic of salutogenic WHP and to focus more on the well-being or mental health of employees instead of only on the physical side of WHP in the future. I recommend SMEs to implement WHP based on salutogenesis. As my study demonstrates, it is important for SMEs to know how health is produced, what counts for maintaining health and how it can be conserved. In other words, it has been confirmed by my study how important it is for SMEs to deal with the emergence of their employees' health instead of managing a system of illness. My study therefore recommends that SMEs strengthen their employees' SoC.

When I remember the start of my thesis, I suspect that the top manager plays a 
role when it comes to WHP in SMEs. Therefore, I formulate the second study research question as follows: How can SMEs develop top management awareness to keep their staff healthy? These assumptions have been confirmed, but the role of the top manager in WHP is more significant than I suspected. With the knowledge gained from my study today, I would focus even more on the top manager and explore his competencies in relation to WHP.

Finally, the aim of my study is to explore salutogenic factors which SMEs in EWL /Germany can use in order to meet their challenges by creating business conditions to keep their employees healthy. It also explored how SMEs can integrate WHP. The theory of salutogenesis is used to give my WHP research a strength-oriented approach, while at the same time placing mental health or wellbeing of employees in the focus. Leaving the pathogenesis behind and asking the question, 'What makes employees healthy?'. SMEs can manage to keep their most important resource, the employee, healthy. That in turn enables SMEs to compete with large companies in innovative WHP and become more attractive employers in their search for professionals and executives. My study achieved fully the aim as it has found an ideal method with the focus group method to get important insights of SMEs regarding health and well-being promotion of workforces. A special focus should be placed on the new salutogenic WHP factors importance of the quality of the relationship between top managers and employees', and the 'importance of the capacity of older top managers to reflect' which my thesis provides as new insights in WHP.

I could come to the conclusion that the salutogenesis is fully applicable in businesses, especially to SMEs in EWL/Germany. The theory of salutogenesis provides SMEs with a theoretical framework to promote workforce health with a focus on well-being. Salutogenesis may lead to a rethinking of SMEs by no longer looking at the symptoms of employees' illnesses, but by focusing on the salutogenic factors that keep their employees healthy. 
This thesis starts and ends with a citation of Selma Langloef as it sums up my journey resulting in this thesis: "The miracle I have been seeking for so long, it is actually myself." 


\section{References}

Advisory Conciliation and Arbitration Service (Acas). (2017). Addressing mental health in the workplace by creating a culture of openeness. Retrieved from https://www.acas.org.uk/media/6083/Case-study---Addressing-mental-health-inthe-workplace-by-creating-a-culture-ofopenness/pdf/Case study Addressing mental health in the workplace by creat ing a culture of openness.pdf:

Ammel, S., Beerheide, E., Georg, A., Lück, P., Müller, M., Schauerte, B., . . . Weimar, A. (2015). KLEIN - GESUND - WETTBEWERBSFÄHIG: Betriebliche Gesundheitsförderung in Kleinbetrieben stärken. Retrieved from http://www.dnbgf.de/fileadmin/downloads/materialien/dateien/DNGF Positionspapi er KMU.pdf

Antonovsky, A. (1979). Health, stress, and coping: San Francisco: Jossey-Bass.

Antonovsky, A. (1987). Unraveling the mystery of health: How people manage stress and stay well: Jossey-bass.

Antonovsky, A. (1996). The salutogenic model as a theory to guide health promotion (Vol. 11). Great Britain: Oxford University Press.

Antonovsky, A. (1997). Zur Entmystifizierung der Gesundheit.

Archer, M. S. (1995). Realist social theory: The morphogenetic approach: Cambridge university press.

Auvinen, A.-M., Kohtamäki, K., \& Ilvesmäki, M., Antti. (2012). Workplace health promotion and stakeholder positions: a Finnish case study. Archives of environmental \& occupational health, 67(3), 177-184.

Badura, B. (2014). The impact of social capital on the health and performance of organizations. In G. F. Bauer \& O. Hämmig (Eds.), Bridging occupational, organizational and public health: A transdisciplinary approach. (pp. 91-100). New York, NY, US: Springer Science + Business Media.

Badura, B., Ducki, A., Schröder, H., Klose, J., \& Meyer, M. (2017). Fehlzeiten-Report 2017: Krise und Gesundheit-Ursachen, Prävention, Bewältigung: Springer-Verlag.

Badura, B., \& Hehlmann, T. (2003). Betriebliche Gesundheitspolitik: Der Weg zur gesunden Organisation: Springer.

Badura, B., Ritter, W., \& Scherf, M. (1999). Betriebliches Gesundheitsmanagement ein Leitfaden für die Praxis.

Banwell, C., Sargent, G., Dixon, J., \& Strazdins, L. (2019). A cultural economy approach to workplace health promotion in Australian small and medium sized workplaces: a critical qualitative study. Critical Public Health, 29(1), 100-109.

Bauer, G., \& Jenny, G. J. (2007). Development, implementation and dissemination of occupational health management (OHM): Putting salutogenesis into practice Occupational health psychology (Vol. 2, pp. 219-250): Ismai.

Bauer, G. F., \& Jenny, G. J. (2012). Moving towards positive organisational health: Challenges and a proposal for a research model of organisational health development. Contemporary occupational health psychology, 2, 126-145.

Berg, B., \& Lune, H. (2004). Qualitative Research Methods for the Social Sciences Pearson Boston.

Bernard, H. R. (1996). Qualitative data, quantitative analysis. CAM Journal, 8(1), 9-11.

Bertelsmann Stiftung. (2011). Zukunftsfähige betriebliche Gesundheitspolitik: Vorschläge der Expertenkommission.

Bertelsmann Stiftung und Hans-Böckler-Stiftung. (2004). Zukunftsfähige betriebliche Gesundheitspolitik. Retrieved from https://www.bertelsmannstiftung.de/de/publikationen/publikation/did/zukunftsfaehige-betrieblichegesundheitspolitik-1/:

Bhaskar, R. (1975). Forms of realism.

Bhaskar, R. (1979). Philosophy and the human sciences: Harvester Press.

Bhaskar, R. (1989). Reclaiming reality: London: Verso. 
Bhaskar, R. (1998). Philosophy and scientific realism. Critical realism: Essential readings, 16-47.

Biallas, B., Schäfer, D., Dejonghe, L., Franz, L., Petrowski, K., Froböse, I., \& Wilke, C. (2018). Präventionsreife in kleinen und mittleren Unternehmen. Prävention und Gesundheitsförderung, 1-5.

Black, C. M. (2008). Working for a healthier tomorrow: Dame Carol Black's review of the health of Britain's working age population: The Stationery Office.

Blättner, B. (2015, 20.06.2019). Salutogenese“ - Leitorientierung für die Entwicklung präventiver Dienstleistungen. https://www.zig-owl.de/wpcontent/uploads/2012/07/entwicklung neuer dienstleistungen fuer praevention $\mathrm{p}$ raesentation 011.pdf.

Bley, A., Vogt, G., Holstein, M., \& Niegsch, C. (2018). Mittelstand im MittelpunktHerbst $2018 . \quad$ Retrieved from https://www.dzbank.de/content/dam/dzbank de/de/home/produkte services/Firme nkunden/PDF-Dokumente/Mittelstandsstudie/Mittelstand-im-Mittelpunkt-Herbst2018.pdf

Blickhan, D. (2015). Positive Psychologie. Ein Handbuch für die Praxis. Paderborn: Junfermann Verlag.

Boatin, J. (2008). Generation als Erzählung. Neue Perspektiven auf ein kulturelles Deutungsmuster. Paper presented at the Seminar für Deutsche Philologie, GeorgAugust-Universität Göttingen.

Booth, A., Clarke, M., Ghersi, D., Moher, D., Petticrew, M., \& Stewart, L. (2011). An international registry of systematic-review protocols. The lancet, 377(9760), 108109.

Brand, S. L., Coon, J. T., Fleming, L. E., Carroll, L., Bethel, A., \& Wyatt, K. (2017). Wholesystem approaches to improving the health and wellbeing of healthcare workers: A systematic review. PloS one, 12(12), e0188418.

Braun, V., \& Clarke, V. (2006). Using Thematic Analysis in Psychology Qualitative Research in Psychology, 3, 77-101. ISI.

Briner, R. B., \& Walshe, N. D. (2015). An evidence - based approach to improving the quality of resource-oriented well-being interventions at work. Journal of occupational and organizational psychology, 88(3), 563-586.

Briner, R.B., Denyer,D., \& Rousseau, D. M. (2009). Evidence-based management: concept cleanup time?. Academy of Management Perspectives, 23(4), 19-32.

Bringsén, Å., Andersson, H. I., Ejlertsson, G., \& Troein, M. (2012). Exploring workplace related health resources from a salutogenic perspective: Results from a focus group study among healthcare workers in Sweden. Work, 42(3), 403-414.

Brinkmann, H.-D. (2015). Woran das BGM oft scheitert.

Brosius, H.-B., Koschel, F., \& Haas, A. (2001). Methoden der empirischen Sozialforschung. Eine Einführung. Wiesbaden.

Bruch, H., \& Kowalevski, S. (2013). Gesunde Führung. Wie Unternehmen eine gesunde Performancekultur entwickeln. Überlingen.

Bruder, R., Luczak, H., \& Schlick, C. (2010). Arbeitswissenschaft: Springer-Verlag, Berlin, Heidelberg.

Bruecker, G., Sochert, R., Jürgens-Scholz, B., Mißler, M., \& Prüßmann, J.-F. (2012). Kein Stress mit dem Stress. Retrieved from https://www.inqa.de/DE/Angebote/Publikationen/psyga-kein-stress-mit-dem-stresshandlungshilfe-beschaeftigte.html:

Bryman, \& Bell. (2007). Business research methods.

Bryman, \& Bell. (2011). Business Research Methods: Oxford University Press Inc., New York.

Buchanan, D., \& Bryman, A. (2009). The Sage handbook of organizational research methods: Sage Publications Ltd.

Bund, K. (2014). Glück schlägt Geld: Generation Y: Was wir wirklich wollen: Murmann Publishers $\mathrm{GmbH}$.

Bundesministerium für Arbeit und Soziales (BMAS). (2014). Sicherheit und Gesundheit bei der Arbeit $2014 . \quad$ Retrieved from 
https://www.baua.de/DE/Angebote/Publikationen/Berichte/Suga2014.pdf? blob=publicationFile\&v=19:

Campbell, R., Pound, P., Pope, C., Britten, N., Pill, R., Morgan, M., \& Donovan, J. (2003). Evaluating meta-ethnography: a synthesis of qualitative research on lay experiences of diabetes and diabetes care. Social science \& medicine, 56(4), 671684.

Cattrell, A., Harris, E. C., Palmer, K. T., Kim, M., Aylward, M., \& Coggon, D. (2011). Regional trends in awards of incapacity benefit by cause. Occupational medicine, 61(3), 148-151.

Center for Evidence Based Management (CEBMa). (2019). Acquiring Evidene From The Scientific Literature https://www.cebma.org/wp-content/uploads/CEBMa-AcquiringEvidene-From-The-Scientific-Literature.pdf: Center for Evidence Based Management (CEBMa).

Chartered Institute of Personnel and Development (CIPD). (2016 ). Growing the health and well-being agendaJanuary 2016. Retrieved from

Chartered Institute of Personnel and Development (CIPD). (2019). HEALTH AND WELLBEING AT WORK. Retrieved from https://www.cipd.co.uk/lmages/health-and-wellbeing-at-work-2019.v1 tcm18-55881.pdf:

Cook, D. J., Mulrow, C. D., \& Haynes, R. B. (1997). Systematic reviews: synthesis of best evidence for clinical decisions. Annals of internal medicine, 126(5), 376-380.

Corbin, J., \& Strauss, A. (2014). Basics of qualitative research: Techniques and procedures for developing grounded theory: Sage publications.

Counsell, C. (1997). Formulating questions and locating primary studies for inclusion in systematic reviews. Annals of internal medicine, 127(5), 380-387.

Cronin, P., Ryan, F., \& Coughlan, M. (2008). Undertaking a literature review: a step-bystep approach. British journal of nursing, 17(1), 38-43.

DAK. (2018). Betriebliches Gesundheitsmanagement.

Daly, J., Kellehear, A., \& Gliksman, M. (1997). The public health researcher: A methodological approach: Melbourne, Australia: Oxford University Press.

Dantzker, M. L., Hunter, R. D., \& Quinn, S. T. (2016). Research methods for criminology and criminal justice: Jones \& Bartlett Learning.

Denyer, D., \& Tranfield, D. (2009). Producing a systematic review. The Sage handbook of organizational research methods, 671-689.

Denzin, N. K., \& Lincoln, Y. S. (2011). The Sage handbook of qualitative research: Sage.

Die Initiative Neue Qualität der Arbeit (INQA). (2017). Psychische Gesundheit in der Arbeitswelt. Kein Stress mit dem Stress. Retrieved from https://www.inqa.de/SharedDocs/PDFs/DE/Projekte/psychische-gesundheitarbeitsweltfaltblatt.pdf;jsessionid=5ABD8A1773F2298402B13B4BFEDFFD4D?_blob=public ationFile \& $v=2$ :

Dixon-Woods, M., \& Fitzpatrick, R. (2001). Qualitative research in systematic reviews: has established a place for itself: British Medical Journal Publishing Group.

Easterby-Smith, M., Thorpe, R., \& Jackson, P. (2012). Management research. London: Sage.

Ejlertsson, L., Heijbel, B., Ejlertsson, G., \& Andersson, I. (2018). Recovery, work-life balance and work experiences important to self-rated health: A questionnaire study on salutogenic work factors among Swedish primary health care employees. Work, 59(1), 155-163.

Elder-Vass, D. (2010). The causal power of social structures: Emergence, structure and agency: Cambridge University Press.

Engeström, Y. (1999). Activity theory and individual and social transformation. Perspectives on activity theory, 19(38).

Eriksson, P., \& Kovalainen, A. (2015). Qualitative methods in business research: A practical guide to social research: Sage.

Esslinger,A.S.,Emmert, M., \& Schöffski, O. (2010). Betriebliches Gesundheitsmanagement: mit gesunden Mitarbeitern zu unternehmerischem Erfolg: Springer-Verlag. 
European Union (EU). (2010). Mental Health and Well-being in Older People - Making it Happen. Paper presented at the EU Thematic conference, Madrid. http://ec.europa.eu/health/mental health/docs/older salutogenesis.pdf, 14.09.2016.

Faltermaier, T. (2012). Salutogenese-Resilienz. Theoretische Grundlagen einer psychosozialen Gesundheitsförderung. Paper presented at the Kerbe. Forum für Psychiatrie.

Federal Association of German Industry e.V. (BDI). (2019). Wo das Herz der Deutschen Wirtschaft schlägt.

Feldt, T., Kinnunen, U., \& Mauno, S. (2000). A mediational model of sense of coherence in the work context: a one - year follow - up study. Journal of Organizational Behavior, 21(4), 461-476.

Fink, A. (2019). Conducting research literature reviews: from the Internet to paper: Sage Publications.

Flick, U., \& Kardorff, E. v. (2012). Handbuch Qualitative Sozialforschung: Grundlagen, Konzepte, Methoden Und Anwendungen. 3., neu ausgestattete Aufl. Ort: Verlag.

Flowers, P. (2009). Research Philosophies - Importance and Relevance https://blogs.warwick.ac.uk/files/cesphd/flowers 2009.pdf.

Franke, F., \& Felfe, J. (2011). Diagnose gesundheitsförderlicher Führung-Das Instrument „Health-oriented Leadership “ Fehlzeiten-Report 2011 (pp. 3-13): Springer.

Franzkowiak, P., Homfeldt, H. G., \& Mühlum, A. (2011). Lehrbuch Gesundheit: Beltz Juventa.

Freeman, S., \& Wilshaw, S. (2007). A focused solution to therapy. Primary Health Care (through 2013), 17(7), 32.

Friczewski, F. (2006). Ein neues Präventionsleitbild psychischer Belastungen am Arbeitsplatz Weißbuch Prävention 2005/2006 (pp. 168-173): Springer.

Ghauri, P. N. (2005). Research methods in business studies: A practical guide: Pearson Education.

Given, L. M. (2008). The Sage encyclopedia of qualitative research methods: Sage Publications.

Glaser, B. G., \& Strauss, A. L. (1998). Grounded theory. Strategien qualitativer Forschung. Bern, 53-84.

Gläser, J., \& Laudel, G. (2009). Wenn zwei das Gleiche sagen... Qualitätsunterschiede zwischen Experten. Experteninterviews. Theorien, Methoden, Anwendungsfelder, 3, 137-158.

Gohs, B. M. (2005). Kooperationen und Netzwerke in der Gesundheitswirtschaft der Region Ostwestfalen-Lippe. Dissertation zur Erlangung des Grades Dr. rer. pol. an der Wirtschaftswissenschaftlichen Fakultät der Universität Paderborn.

Gordon, R. L. (1975). Interviewing: Strategies, Techniques and Tactics (Vol. III). Chicago.

Gorski, P. S. (2008). The ECPRES Model: A Critical Realist Approach to Causal Mechanisms in the Social Sciences. The Frontiers of Sociology, 147-194.

Grabert, A. (2011). Salutogenese und Bewältigung psychischer Erkrankung: Einsatz des Kohärenzgefühls in der sozialen Arbeit: Jacobs-Verlag.

Grawitch, M. J., Trares, S., \& Kohler, J. M. (2007). Healthy workplace practices and employee outcomes. International Journal of Stress Management, 14(3), 275.

Greasley, K., Edwards, P., Baker-McClearn, D., \& Dale, J. (2012). Why do organisations engage in HR initiatives? A test case of a health and wellbeing intervention. Employee Relations, 34(4), 443-462.

Gregersen, S., Vincent-Höper, S., \& Nienhaus, A. (2014). Health-relevant leadership behaviour: A comparison of leadership constructs. German Journal of Human Resource Management, 28(1-2), 117-138.

Grix, J. (2002). Introducing students to the generic terminology of social research. Politics, 22(3), 175-186.

Gröben, F. D. (2008). Betriebliche Gesundheitsförderung in mittelständischen Unternehmen. Prävention und Gesundheitsförderung, 3(4), 227-234. doi:10.1007/s11553-008-0137-x 
Guba, E. G., \& Lincoln, Y. S. (1994). Competing paradigms in qualitative research Handbook of qualitative research (Vol. 105, pp. 163-194).

Guest, G., MacQueen, K. M., \& Namey, E. E. (2012). Applied thematic analysis: Sage.

Gurt, J., Schwennen, C., \& Elke, G. (2011). Health-specific leadership: is there an association between leader consideration for the health of employees and their strain and well-being? Work \& Stress, 25(2), 108-127.

Haddaway, N., Woodcock, P., Macura, B., \& Collins, A. (2015). Making literature reviews more reliable through application of lessons from systematic reviews (Vol. 29).

Hart, C. (2001). Doing a literature search: a comprehensive guide for the social sciences: Sage.

Harvey, B. S., Joyce, S., Tan, L., Johnson, A., Nguyen, H., Modini, M., \& Groth, M. (2014). Developing a mentally healthy workplace: $A$ review of the literature.

Harzer, C., \& Möller, H. (2017). Positive Psychologie und ihre Bedeutung in der Beratung. Organisationsberatung, Supervision, Coaching, 24(3), 247-252. doi:10.1007/s11613-017-0511-7

Hauser, F., \& Pleuger, F. (2010). Great Place to Work®: Ein Arbeitsplatz, an dem man sich wohl fühlt Fehlzeiten-Report 2009 (pp. 197-204): Springer.

Henning, R. A., Reeves, D. W., \& Team, C.-N. R. (2013). An integrated health protection/promotion program supporting participatory ergonomics and salutogenic approaches in the design of workplace interventions Salutogenic organizations and change (pp. 307-325): Springer.

Heyse, V., Erpenbeck, J., \& Max, H. (2004). Kompetenzen erkennen, bilanzieren und entwickeln: Waxmann Verlag.

Higgins, J. P., \& Green, S. (2011). Cochrane handbook for systematic reviews of interventions (Vol. 4): John Wiley \& Sons.

Holt, M., \& Powell, S. (2015). Health and well-being in small and medium-sized enterprises (SMEs). What public health support do SMEs really need? Perspectives in public health, 135(1), 49-55.

Homfeldt, H. G., \& Sting, S. (1997). Soziale Arbeit und Gesundheit: Luchterhand.

Hopkins, T., \& Rippon, S. (2015). Head, hands and heart: asset-based approaches in health care.2016. Retrieved from http://www.colabdudley.net/wpcontent/uploads/2015/11/headhandsandheartassetbasedapproachesinhealthcare.p df

Hutt, R. W. (1979). The focus group interview: A technique for counseling small business clients. Journal of Small Business Management (pre-1986), 17(000001), 15.

Initiative neue Qualität der Arbeit (INQA). (2014). Führungskultur im Wandel. Retrieved from

Initiative New Quality of Work (INQA). (2019). INQA Unternehmenscheck "Guter Mittelstand". https://www.ingaunternehmenscheck.de/check/daten/mittelstand/index.htm.

Institute for SME Research (IFM). (2019, 25.01.2019). Informationen zur mittelständischen Wirtschaft aus erster Hand

James, J. B., Swanberg, J. E., \& McKechnie, S. P. (2007). Generational differences in perceptions of older workers' capabilities: Center on Aging \& Work/Workplace Flexibility at Boston College.

Janis, I. L. (1972). Victims of groupthink: a psychological study of foreign-policy decisions and fiascoes.

Jankowicz, A. D. (2005). Business research projects: Cengage Learning EMEA.

Jensen, L. A., \& Allen, M. N. (1996). Meta-synthesis of qualitative findings. Qualitative health research, 6(4), 553-560.

Jonassen, D. H. (1991). Objectivism versus constructivism: Do we need a new philosophical paradigm? Educational technology research and development, 39(3), 5-14.

Joseph, S., \& Sagy, S. (2017). Positive psychology in the context of salutogenesis The handbook of salutogenesis (pp. 83-88): Springer, Cham.

Kane, S. (2018). Workplace: How Their Generational Traits and Characteristics Affect the Workplace. 
Khan, K. S., Ter Riet, G., Glanville, J., Sowden, A. J., \& Kleijnen, J. (2001). Undertaking systematic reviews of research on effectiveness: CRD's guidance for carrying out or commissioning reviews: NHS Centre for Reviews and Dissemination.

Kiesche, E. (2013). Betriebliches Gesundheitsmanagement: Bund-Verlag.

Kitzinger, J. (1995). Qualitative research: introducing focus groups. Bmj, 311(7000), 299302.

Krueger, R. A., \& Casey, M. A. (2014). Focus groups: A practical guide for applied research: Sage publications.

Lavrakas, P. J. (2008). Encyclopedia of survey research methods: Sage Publications.

Lawson, T. (1997). Economics and reality.

Leung, L. (2015). Validity, reliability, and generalizability in qualitative research. Journal of family medicine and primary care, 4(3), 324.

Lewis, R., Donaldson-Feilder, E., Jones, B., \& Johal, M. (2014). Developing managers to manage sustainable employee engagement, health and well-being: phase 1.

Lindström, B., \& Eriksson, M. (2005). Salutogenesis. Journal of Epidemiology and community health, 59(6), 440-442.

Lüerßen, H., Stickling, E., Gundermann, N., Toska, M., Coppik, R., Denker, P., . . . Timmerhoff, C. (2015). BGM im Mittelstand 2015. Retrieved from https://www.iasgruppe.de/fileadmin/user upload/Presse und Publikationen/Presseberichte und Studien/PW Studie 102015 web.pdf:

Lyssenko, L., Franzkowiak, P., \& Bengel, J. (2011). Resilienz und Schutzfaktoren. Leitbegriffe der Gesundheitsförderung und Prävention. Glossar zu Konzepten, Strategien und Methoden. Neuausgabe, 476-478.

MacQueen, K. M., \& Milstein, B. (1999). A systems approach to qualitative data management and analysis. Field Methods, 11(1), 27-39.

Madill, A., Jordan, A., \& Shirley, C. (2000). Objectivity and reliability in qualitative analysis: Realist, contextualist and radical constructionist epistemologies. British journal of psychology, 91(1), 1-20.

Mannheim, K. (1970). The problem of generations. Psychoanalytic review, 57(3), 378-404.

Marczak, M., \& Sewell, M. (1991). Using focus groups for evaluation. Cybernet Evaluation. Tuscon, AZ: The University of Arizona.

Marshall, C., \& Rossman, G. B. (2014). Designing qualitative research: Sage publications.

Martin, A., Sanderson, K., Scott, J., \& Brough, P. (2009). Promoting mental health in smallmedium enterprises: An evaluation of the" Business in Mind" program. BMC Public Health, 9(1), 239.

Matyssek, A. K. (2012). Führung und Gesundheit: ein praktischer Ratgeber zur Förderung der psychosozialen Gesundheit im Betrieb: BoD-Books on Demand.

May-Strobl, E., \& Welter, F. (2015). Das Zukunftspanel Mittelstand

IfM - Materialien Nr. 239.

McDermott, E., Graham, H., \& Hamilton, V. (2004). Experience of being a Teenage Mother in the UK: A Report of systematic review of qualitative studies.

Merton, R. K. (1967). On theoretical sociology: Free Press New York.

Mette, J., \& Harth, V. (2017). Das Kohärenzgefühl im Arbeitskontext. Zentralblatt für Arbeitsmedizin, Arbeitsschutz und Ergonomie, 67(4), 240-244. doi:10.1007/s40664-017-0168-2

Meyer, J. A. (2008). Gesundheit in KMU. Retrieved from http://www.gesundheitsbewussterbetrieb.de/pdf/Gesundheit in KMU.pdf:

Miles, M., \& Huberman, A. (1991). Designing qualitative research. Mac Graw Hill Company. New York.

Miles, M. B., \& Huberman, A. M. (1994). Qualitative data analysis: An expanded sourcebook: Sage.

Mittelmark, M., Sagy, S., Eriksson, M., Bauer, G. F., M., P. J., Lindström, B., \& Espnes, G. A. (2016). The Handbook of Salutogenesis. Heidelberg: Springer.

Mohagheghi, P., \& Conradi, R. (2004). Vote-counting for combining quantitative evidence from empirical studies-an example. Paper presented at the Proceedings of the International Symposium on Empirical Software Engineering (ISESE'04). 
Moore, A., Parahoo, K., \& Fleming, P. (2010). Workplace health promotion within small and medium-sized enterprises. Health Education, 110(1), 61-76.

Moore, A., Parahoo, K., \& Fleming, P. (2011). Managers' understanding of workplace health promotion within small and medium-sized enterprises: A phenomenological study. Health Education Journal, 70(1), 92-101.

Morgan. (1988). Focus groups as qualitative research. University Paper Series on Qualitative Research Methods: Newbury Park CA.: Sage.

Morgan. (1996). Focus Groups. Annual Review of Sociology, 22, pp. 129-152.

Murray, C. J., Vos, T., Lozano, R., Naghavi, M., Flaxman, A. D., Michaud, C., . . Abdalla, S. (2012). Disability-adjusted life years (DALYs) for 291 diseases and injuries in 21 regions, 1990-2010: a systematic analysis for the Global Burden of Disease Study 2010. The lancet, 380(9859), 2197-2223.

Myers, M. D. (2013). Qualitative research in business and management: Sage.

Nilsson, P. (2010). Enhance your workplace!: a dialogue tool for workplace health promotion with salutogenic approach. Institution of Clinical Science, Malmö, Lund University.

Nilsson, P., Andersson, I. H., Ejlertsson, G., \& Troein, M. (2012). Workplace health resources based on sense of coherence theory. International Journal of Workplace Health Management, 5(3), 156-167.

Nkwi, P. N., Nyamongo, I. K., \& Ryan, G. W. (2001). Field research into socio-cultural issues: Methodological guidelines: International Center for Applied Social Sciences, Research, and Training.

Noblit, G. W., \& Hare, R. D. (1988). Meta-ethnography: Synthesizing qualitative studies (Vol. 11): sage.

Nyberg, A. (2009). The impact of managerial leadership on stress and health among employees: Institutionen för folkhälsovetenskap/Department of Public Health Sciences.

Office for National Statistics (2015). Statistisches Jahrbuch Deutschland und Internationales.

Oliver, S., Harden, A., Rees, R., Shepherd, J., Brunton, G., Garcia, J., \& Oakley, A. (2005). An emerging framework for including different types of evidence in systematic reviews for public policy. Evaluation, 11(4), 428-446.

Orb, A., Eisenhauer, L., \& Wynaden, D. (2001). Ethics in qualitative research. Journal of nursing scholarship, 33(1), 93-96.

Ormston, R., Spencer, L., Barnard, M., \& Snape, D. (2014). The foundations of qualitative research. Qualitative research practice: A guide for social science students and researchers, 2.

Pawson, R. (2006). Evidence-based policy: a realist perspective: Sage.

Pawson, R., \& Tilley, N. (2004). Realist Evaluation.

Petticrew, M., \& Roberts, H. (2008). Systematic reviews in the social sciences: A practical guide: John Wiley \& Sons.

Polonsky, M. J., \& Waller, D. S. (2010). Designing and managing a research project: $A$ business student's guide: Sage.

Popay, J., Roberts, H., Sowden, A., Petticrew, M., Arai, L., Rodgers, M., . . . Duffy, S. (2006). Guidance on the conduct of narrative synthesis in systematic reviews. $A$ product from the ESRC methods programme Version, 1, b92.

Porpora, D. V. (2015). Reconstructing sociology: The critical realist approach: Cambridge University Press.

PricewaterhouseCoopers. (2014). Creating a mentally healthy workplace Return on investment analysis. Retrieved from https://www.headsup.org.au/docs/defaultsource/resources/beyondblue workplaceroi finalreport may-2014.pdf:

Pundt, J., \& Scherenberg, V. (2016). Erfolgsfaktor Gesundheit in Unternehmen: Zwischen Kulturwandel und Profitkultur (Vol. 2): BoD-Books on Demand.

Ramirez, R., Mukherjee, M., Vezzoli, S., \& Kramer, A. M. (2015). Scenarios as a scholarly methodology to produce "interesting research". Futures, 71, 70-87. doi:http://dx.doi.org/10.1016/j.futures.2015.06.006

Rau, R. (2015). Risikobereiche für psychische Belastungen. . Retrieved from 
Reed, M. I. (2009). Critical Realism: Philosophy, Method, or Philosophy in Search of a Method? In S. Handbook (Ed.), Organizational Research Methods (pp. 430-448): SAGE.

Ridley, D. (2012). The literature review: A step-by-step guide for students: Sage.

Riess, B. (2013). Zukunft unternehmen: Bertelsmann Stiftung

Röhl, K.-H. (2017). Europäische Mittelstandspolitik. (Institut der deutschen Wirtschaft Köln).

Ryan, G. W., \& Bernard, H. R. (2010). Analyzing qualitative data: Systematic approaches: Thousand Oaks: Sage.

Saksvik, P. Ø., Olaniyan, O. S., Lysklett, K., Lien, M., \& Bjerke, L. (2015). A process evaluation of a salutogenic intervention. Scandinavian Psychologist, 2.

Sandelowski, M., Docherty, S., \& Emden, C. (1997). Focus on qualitative methods Qualitative metasynthesis: issues and techniques. Research in nursing and health, 20, 365-372.

Sattes, I., Brodbeck, H., \& Lang, H. (1998). Erfolg in kleinen und mittleren Unter-nehmen (Success in small and medium-sized companies). Domeisen, $H$ (Publisher), second edition, Zurich.

Saunders. (2011). Research methods for business students, 5/e: Pearson Education India.

Saunders, Lewis, \& Thornhill. (2011). Research Methods For Business Students, 5/e: Pearson Education India.

Sayer, A. (2010). Method in social science: revised 2nd edition: Routledge.

Schwartz, M., \& Gerstenberger, J. (2018). KfW-Mittelstandsatlas 2018. Retrieved from https://www.kfw.de/PDF/Download-Center/Konzernthemen/Research/PDFDokumente-KfW-Mittelstandsatlas/Mittelstandsatlas-2018/KfWMittelstandsatlas 2018.pdf

Seligman, M. (2017). Opening Keynote auf der 5. Weltkonferenz zur Positiven Psychologie in Montreal, 2017.

Shepherd, J., Harden, A., Rees, R., Brunton, G., Garcia, J., Oliver, S., \& Oakley, A. (2006). Young people and healthy eating: a systematic review of research on barriers and facilitators. Health Education Research, 21(2), 239-257.

Shiels, C., Gabbay, M. B., \& Ford, F. M. (2004). Patient factors associated with duration of certified sickness absence and transition to long-term incapacity. $\mathrm{Br} J$ Gen Pract, 54(499), 86-91.

Silverman, D. (2013). Doing qualitative research: A practical handbook: SAGE Publications Limited.

Singer, S., \& Brähler, E. (2007). Die» Sense of Coherence Scale «: Testhandbuch zur deutschen Version: Vandenhoeck \& Ruprecht.

Slater, P. (1958). Contrasting correlates of group size. Sociometry, 21, 129-139.

Stewart, D. W., Shamdasani, P. N., \& Rook, D. W. (1990). Focus groups: Theory and practice. Applied social research methods series. Focus groups: theory and practice applied social research methods series.

Stickley, T., \& Hoare, M. (2015). A review of the concept of Salutogenesis and its significance for promoting mental health recovery through participatory arts. Journal of Applied Arts \& Health, 6(1), 63-75.

Tang , K. C., \& Davis, A. (1995). Critical factors in the determination of focus group size. Familiy Practice, 12, Nr. 4.

The Federal Institute for Occupational Safety and Health (BAuA). (2018). Gefährdungsbeurteilung psychischer Belastung. Forschung und Entwicklung für die betriebliche Praxis. Retrieved from https://www.baua.de/DE/Themen/Arbeitsgestaltung-im-Betrieb/PsychischeBelastung/Gefaehrdungsbeurteilung/Gefaehrdungsbeurteilung node.html

The National Association of Statutory Health Insurance Funds. (2017). Betriebliche Gesundheitsförderung.

Tödtmann, C. (2011). So führt der Mittelstand. https://www.wiwo.de/unternehmen/managementstudie-so-fuehrt-dermittelstand/5289686-all.html. 
Tranfield, D., Denyer, D., \& Smart, P. (2003). Towards a methodology for developing evidence - informed management knowledge by means of systematic review. British journal of management, 14(3), 207-222.

University of Gloucestershire. (2008). Reserach Ethics: A Handbook of Principles and Procedures: University of Gloucestershire.

Vincent, S. (2011). Gesundheits-und entwicklungsförderliches Führungsverhalten: ein Analyseinstrument Fehlzeiten-Report 2011 (pp. 49-60): Springer.

Vinje, H. F., \& Ausland, L. H. (2013). Salutogenic presence supports a healthpromoting work life. Socialmedicinsk tidskrift, 90(6), 890-901.

Voermans, S. (2016). Entspann dich, Deutschland - TK-Stressstudie 2016. Retrieved from https://www.tk.de/resource/blob/2026630/9154e4c71766c410dc859916aa798217/t k-stressstudie-2016-data.pdf:

Vogt, K., Hakanen, J. J., Jenny, G. J., \& Bauer, G. F. (2016). Sense of coherence and the motivational process of the job-demands-resources model. Journal of Occupational Health Psychology, 21(2), 194.

Vogt, K., Jenny, G. J., \& Bauer, G. F. (2013). Comprehensibility, manageability and meaningfulness at work: Construct validity of a scale measuring work-related sense of coherence. SA Journal of Industrial Psychology, 39(1), 1-8.

Watts $M$, Ebbutt D. More than the sum of the parts: research methods in group interviewing. British Educational Research Journal 1987;13:25-34.

Wertsch, J. V. (1986). Culture, communication, and cognition: Vygotskian perspectives: CUP Archive.

Whetten, D. A. (1989). What constitutes a theoretical contribution? Academy of management review, 14(4), 490-495.

Wiedmer, T. (2015). Generations do differ: Best practices in leading traditionalists, boomers, and generations X, Y, and Z. Delta Kappa Gamma Bulletin, 82(1), 51.

Williams, B. (2017). Qualitative Data Analysis Retrieved from http://www.bobwilliams.co.nz/ewExternalFiles/analysis2.pdf

Williams, S. J., \& Snow, D. M. (2012). Promoting health in small and medium-sized enterprises. Journal of Small Business and Enterprise Development, 19(4), 729744.

Wolff, A. C., \& Ratner, P. A. (1999). Stress, social support, and sense of coherence. Western Journal of Nursing Research, 21(2), 182-197.

Wollesen, B., Menzel, J., Drögemüller, R., Hartwig, C., \& Mattes, K. (2017). The effects of a workplace health promotion program in small and middle-sized companies: a pre-post analysis. Journal of public health, 25(1), 37-47.

Wong, M., Gardiner, E., Lang, W., \& Coulon, L. (2008). Generational differences in personality and motivation: do they exist and what are the implications for the workplace? Journal of Managerial Psychology, 23(8), 878-890.

World Health Organization (WHO). (1986). Ottawa charter for health promotion.

World Health Organization (WHO). (2005). Global health promotion scaling up for 2015 - A brief review of major impacts and developments over the past 20 years and challenges for 2015. (WHO Secretariat Background Document for the 6th Global Conference on Health Promotion in Bangkok, Thailand 7-11 August 2005.).

World Health Organization (WHO) Regional Committee for Europe. (2013). Fact sheet Mental health. 63rd session,

http://www.euro.who.int/ data/assets/pdf file/0004/215275/RC215263-Factsheet-MNH-Eng.pdf?ua=215271.

Wydler, H., Kolip, P., \& Abel, T. (2010). Salutogenese und Kohärenzgefühl, Grundlagen, Empirie und Praxis eines gesundheitswissenschaftlichen Konzepts, 4. Auflage, 2010: Weinheim und München: Juventa Verlag.

Zimber, A. (2011). Wie Führungskräfte zur Mitarbeitergesundheit beitragen können: Eine Pilotstudie in ausgewählten BGW-Mitgliedsbetrieben.

Zimber, A., \& Gregersen, S. (2011). Gesundheitsfördend führen Fehlzeiten-Report 2011: Springer. 


\section{Appendix - table of content}

9.1 Nine recommendations within the study by Bruch et al. (2013) 200

9.2 Orginal of the guidline within the study by Bruch et al. (2013) 204 (short version in German language)

9.3 Studies which met the quality assessment criteria

9.4 Final studies with information after the first synthesis step

9.5 Final studies with information after the second synthesis step

9.6 Example of code table

9.7 Grading system assessing the robustness of the synthesis

9.8 Example of 'Participant Information Sheet' (PIS) 219

9.9 Codebook

9.10 Partial of focus-group transcripts

9.10.1 Focus-group discussion top managers

9.10.2 Focus-group discussion employees

9.10.3 Reflection focus-group discussion

9.11 Slides of the focus-group discussions 


\subsection{Nine recommendations for health promotion within the study by Bruch et al.}

\section{(2013) (translated by a translation agency).}

\section{Top management as pioneers of a healthy performance culture}

A company's top management is central to a healthy performance culture. Managing directors are internal trendsetters, who can use their role to initiate, promote and consolidate a healthy performance culture. Conversely, top managers who do not take the issue of health seriously and set a bad example can undo all of other people's efforts, since employees feel that the measures are insincere or just a case of a company covering itself. By taking care of their own health, management signals to executives and employees that health is an important issue in the company. Healthy leadership at the top leadership level spreads throughout the company, which means that healthy leadership becomes sustainably positive at all levels.

TeamBank AG (financial services provider, 1,000 employees, based in Nuremberg)

However, top executives should pay attention not only to their own health, but also develop a sense of responsibility for the health of their employees, e.g. by recognising and responding to warning signals at an early stage.

\section{Healthy self-management}

Executives have a classic dual role, especially when it comes to health. However, the majority of top managers, do not see "self-care" as a key issue. Instead of a "I take care of myself", top managers are more likely to say "there is more asked of me than of my co-workers". Top managers adopting this attitude, and the behaviour to which it leads, is not only harmful to their own health, but is also harmful to the health of the employees. Employees take note of the fact that management are being inconsistent, if they suggest to employees that the latter stay at home until they are better, but the former come to work ill themselves. For the employees, this behaviour makes it seem as if the executives have an implicit and unspoken expectation that they will ignore their health problems and personal sensitivities and still come to work. The credibility of leadership is greatly affected by this. Therefore, top managers should first of all manage themselves in a healthy manner. In this way, they inspire confidence and show consistency when they also manage their employees in a healthy manner.

\section{Holistic health management}

In many companies, workplace health management is still in its infancy. The various measures, which are brought together in companies under the heading of "occupational health management", are more effective at promoting health when they are interlinked and tailored specifically to the needs of the company and its employees. However, holistic health management still boils down to two major steps.

Firstly, the development of management is pivotal to effective holistic company health management. Training and leadership simulations can be used as a means to learn and practice good leadership in general and healthy leadership in particular. The transfer of what is learned in leadership training into everyday life will, however, only be successful if good guidance is initiated and practised at senior levels. A management team which performs well has a positive influence on the behaviour of the top managers who report into them and this in turn influences the management style of those working in the next level down.

Secondly, holistic company health management encompasses preventive and interventional measures at both individual and organisational levels. An example of such preventive and intervention measures at an organisational level could be measures to avoid and overcome acceleration traps. Working to create a rhythm between phases of high performance and phases of regeneration, the establishment of time-outs and moments for reflection as part of company culture and/or systematic project selection are essential components of holistic company health management.

B.A.D. Gesundheitsvorsorge und SicherheitstechnikGmbH (prevention services in the field of health, 1,900 
employees, based in Bonn)

Organisational changes (e.g. the standardisation of procedures, an organisational telephone service, optimised scheduling, definition of responsibilities, improvement of the archiving of files, etc.)

Structural changes (e.g. providing relief to overloaded assistants in service centres with back- and front office activities by the spatial separation of work processes, optimisation of the function rooms, expansion of the premises/space requirement)

Personnel changes (e.g. recruitment of additional staff, adjustment of capacities, checking of resources, optimisation of tasks, etc.)

Changes in equipment (e.g. upgrading/improving hardware, acquisition of height-adjustable desks/consoles, procurement of equipment for transport, acquisition of new medical equipment, acquisition of navigational equipment, improvement of telephone systems etc.)

Changes in communication and information flow (e.g. improvement of regular team meetings and internal communication tools)

Promotion of team spirit (e.g. organisation of company excursions, coaching team, organisation of team training evening, seminars in conflict management and time management)

As a secondary effect of their internal development of measures for the diagnosis of psychological stresses, B.A.D. Gesundheitsvorsorge und Sicherheitstechnik $\mathrm{GmbH}$ created an analytical tool for the diagnosis of psychological stress, which they put on the market. In this case, the focus on mental health paid twice over.

\section{Freedom with a safety net}

The delegation and acceptance of responsibilities are both crucial for management and employees to meet increased requirements. The transfer of responsibility means management letting go of a responsibility and the employee taking it over, so the management needs to be able to trust them and have confidence in them. Working independently in a trusting environment has a positive effect on the health of the employees and also has a strong ripple effect in different areas of life. In this way, employees who are given a great deal of room for manoeuvre in the working context are better able to deal with their resources and their health better. At the same time, a leader should provide a safety net for their employees if they need support. Responsible transfer also means saying "no" to a superior or to the customer when it is necessary for the health and efficiency of the employee. Abat AG (IT development and consulting, 191 employees, based in Bremen)

\section{Meaningful work}

It is important that employees know the importance of their contribution to the achievement of company goals. Particularly in highly specialised companies, employees can find little to no connection between their tasks and the end product. This will raise major questions about the meaningfulness of one's own work. A person's work will increasingly be perceived as unimportant, boring and interchangeable - an impression which, over time, will come to influence the person and result in the employee feeling detached from their own tasks and from the company as a whole, which can end in a crisis of personal identity and significance. This leads to a greater likelihood of exhaustion or depression. Executives can counteract this by clearly communicating the vision of the company to their employees and making clear how they contribute to achieving these goals. SwagelokMunchen | B.E.S.T. Fluidsysteme GmbH Munich (plant construction, 25 employees, based in Garching)

\section{Value}

According to the WHO (World Health Organization), "health is a state of complete physical, mental and social well-being and not just the absence of disease or infirmity". Recognition and appreciation of one's own person are important for spiritual and social well-being and this goes beyond praise for very good achievements. The feeling of being worth something and of having achieved something of value has a 
positive effect on well-being and creates a deep sense of satisfaction with oneself, self-confidence in one's abilities and ultimately has a beneficial effect on health.

KiKxxl GmbH (communication service provider, 1,200 employees, based in Osnabrück)

In the summer of 2012, for example, KiKxxI made changes to the names of all functional levels: "Call centre agent" became "customer consultant" to show people internally and externally that the employees were appreciated.

In addition, praise cards were printed and distributed and the executives were encouraged to give these to their employees if they had done something remarkable.

Furthermore, a shadowing model was initiated, the bonus system was revised and made more attractive, a detailed employee-development programme was introduced and various other comprehensive measures were initiated. As an emotional incentive, for example, a shoe flat rate, a cinema flat rate or a music flat rate were awarded to the customer consultants for one year each.

Moreover, all conference rooms, which until that point had only been numbered, were renamed to the five corporate values: respect, creativity, responsibility, quality and teamwork. That is why we have been meeting in the "Quality Room" or the "Creativity Room" since last year.

KiKxxl classed all these measures under the heading: "Turning employees into fans". As a result of this initiative, in $2012 \mathrm{KiKxxl}$ became the only call centre to be certified by TÜV Süd in the field of employee orientation

\section{Challenging and supporting}

The roots of occupational health management lie in occupational health and safety and occupational safety.

The realisation that working conditions are important to the health of employees and companies have a duty to work in the field of occupational safety has been an important milestone in the development of occupational health management. For the mental health of employees, the work content is an important part. Demanding and diversified activities, for which the employees have the appropriate skills, are personal and health promoting. Employees can then develop themselves and gain self-confidence in their own abilities. The level of skills and abilities are very different from person to person. Executives should therefore pay attention to distributing tasks so that the resources of the employees are in harmony with the level of skills and abilities. The perceived development perspectives play a key role here. In employee surveys, it is often the case that employees want more development opportunities. Here, perspective is a decisive factor for performance, intrinsic motivation and health.

\section{8. "Health consumption"}

Management, HR and executives have a duty to provide care for the employees, in particular for their wellbeing and health. However, every employee is responsible for his or her own health and must not be relieved of this responsibility due to misunderstood understanding of consumption in the sense of "I'm waiting to see what my management offers me."

Employees tend to see the promotion of their health the sole responsibility of their leadership, and thus often have unrealistic expectations. Employees should have primary responsibility for their own health. For example, employees can actively point out potential problems and give feedback to their management, which is good for them, and thus identify, together with their top manager, possible early warning signals, which should be taken seriously.

\section{Psychological "immune system"}

Executives should shape the work environment of their employees so that requirements are in proportion to resources. Employees can, however, do a lot to improve their personal resilience and immunity to stress triggers. Exercises which build and promote resilience, which is to say resistance, are particularly helpful here. This includes completing the day on a positive note by praising an employee in front of the workplace 
for two or three things which they did well on that day. Many employees set up to-do lists per day or week and find themselves frustrated after the end of the day or week that they only managed to complete a fraction of what they had intended to do. It is therefore healthy to keep an actual list, in which employees note what they have been able to do and have checked off. This strengthens self-confidence and motivates them to undertake the new tasks which have not yet been completed. SwagelokMunchen | B.E.S.T. Fluidsysteme GmbH Munich (Plant construction, 25 employees, based in Garching) 


\section{Neun Maßnahmen für eine gesunde Performancekultur}

\section{Top-Management als Vorreiter \\ 1. einer gesunden Performancekultur}

Geschäftsführungsmitglieder sind interne Trendsetter, die in ihrer Rolle eine gesunde Performancekultur anstoßen, fördem und festigen können.

\section{Gesunde Selbstführung}

Führungskräfte sollten sich zunächst selbst gesund führen. Damit schaffen sie Vertrauen und sind authentisch in ihrer gesunden Führung der Mitarbeiter.

\section{Ganzheitliches Betriebliches}

\section{Gesundheitsmanagement}

Die verschiedenen Maßnahmen entfalten einen stărkeren Effekt, wenn sie miteinander verzahnt und spezifisch auf das Unternehmen und seine Mitarbeiter zuges chnitten sind.

\section{Freiheit mit Auffangnetz}

Mitarbeiter, denen ein großer Handlungsspielraum gewährt wird, schaffen es besser, mit ihren Ressourcen und ihrer Gesundheit umzugehen, als andere. Gleichzeitig sollten Führungskräfte ein Auffangnetz anbieten, wenn Unterstützung gefragt ist.

\section{Sinn in der Arbeit}

Gerade in hochspezialisierten Unternehmen können Mitarbeiter ihre Aufgaben nur wenig mit den Endprodukten in Verbindung bringen. Die Motivation sinkt. Führungs kräfte sollten deshalb die Vision des Unternehmens klar kommunizieren und den Mitarbeitern ihren Beitrag dazu verdeut lichen.

\section{- Wertschätzung}

Für das geistige und soziale Wohlergehen sind Anerkennung und Wertschätzung über das Loben für sehr gute Leistungen hinaus bedeutsam. Eine Kultur der Wertschătzung hat deshalb einen gesundheitsförderlichen Effekt.

\section{Fordern und Fördern}

Führungskräfte sollten darauf achten, Aufgaben so zu verteilen, dass die Ressourcen der Mitarbeiter mit dem Anspruchsniveau und ihren Fähigkeiten in Einklang stehen. Eine Schlüsselrolle spielen hierbei die wahrgenommenen Entwicklungsperspektiven.

\section{- "Gesundheits-Konsumhaltung“}

Manche Mitarbeiter neigen dazu, die Förderung ihrer Gesundheit zur alleinigen Aufgabe ihrer Führungskraft zu machen. Stattdessen sollten sie die Verantwortung für die eigene Gesundheit selbst in die Hand nehmen.

Psychisches „Immunsystem“ Führungskräfte sollten das Arbeitsumfeld ihrer Mitarbeiter so gestalten, dass die Anforderungen nicht die Ressourcen übersteigen. Mitarbeiter können jedoch eine ganze Menge selbst dafür tun, sich widerstandsfăhig und immun gegen Stressoren zu machen. 


\subsection{Studies which met the quality assessment criteria.}

\begin{tabular}{|c|c|c|c|c|c|c|}
\hline No. & Authors & Place, time & Title & Rate & $\begin{array}{l}\text { Samples' } \\
\text { environment }\end{array}$ & Methodology \\
\hline 1 & $\begin{array}{l}\text { Williams, S. J. } \\
\text { Snow, D. M. }\end{array}$ & UK, 2012 & $\begin{array}{l}\text { Promoting health in small } \\
\text { and medium-sized } \\
\text { enterprises }\end{array}$ & ABCE & University & $\begin{array}{l}\text { Qualitative } \\
\text { approach, } \\
\text { interviews, } \\
\text { thematic content } \\
\text { analysis }\end{array}$ \\
\hline 2 & $\begin{array}{l}\text { Moore, A. } \\
\text { Parahoo, K. } \\
\text { Fleming, P. }\end{array}$ & UK, 2010 & $\begin{array}{l}\text { Workplace health } \\
\text { promotion within small } \\
\text { and medium-sized } \\
\text { enterprises }\end{array}$ & ABCE & University & $\begin{array}{l}\text { Qualitative } \\
\text { approach, } \\
\text { interviews, } \\
\text { Benner's strategy } \\
\text { for data analysis }\end{array}$ \\
\hline 3 & $\begin{array}{l}\text { Banwell, C. } \\
\text { Sargent, G. } \\
\text { Dixon, J. } \\
\text { Strazdins, L. }\end{array}$ & $\begin{array}{l}\text { Australia, } \\
2019\end{array}$ & $\begin{array}{l}\text { A cultural economy } \\
\text { approach to workplace } \\
\text { health promotion in } \\
\text { Australian small and } \\
\text { medium- sized } \\
\text { workplaces: a critical } \\
\text { qualitative study }\end{array}$ & ABCE & University & $\begin{array}{l}\text { Qualitative } \\
\text { approach, } \\
\text { interviews, } \\
\text { thematic content } \\
\text { analysis }\end{array}$ \\
\hline 4 & $\begin{array}{l}\text { Moore, A. } \\
\text { Parahoo, K. } \\
\text { Fleming, P. }\end{array}$ & UK, 2011 & $\begin{array}{l}\text { Managers' understanding } \\
\text { of workplace health } \\
\text { promotion within small } \\
\text { and medium-sized } \\
\text { enterprises: A } \\
\text { phenomenological study. }\end{array}$ & ABCE & University & $\begin{array}{l}\text { Qualitative } \\
\text { approach, } \\
\text { interviews, } \\
\text { Benner's strategy } \\
\text { for data analysis }\end{array}$ \\
\hline 5 & $\begin{array}{l}\text { Holt, M. } \\
\text { Powell, S. }\end{array}$ & UK, 2015 & $\begin{array}{l}\text { Health and well-being in } \\
\text { small and medium-sized } \\
\text { enterprises (SMEs). What } \\
\text { public health support do } \\
\text { SMEs really need? }\end{array}$ & ABCE & University & $\begin{array}{l}\text { Qualitative } \\
\text { approach, } \\
\text { interviews, } \\
\text { thematic content } \\
\text { analysis }\end{array}$ \\
\hline 6 & $\begin{array}{l}\text { Auvinen, A.-M. } \\
\text { Kohtamäki, K. } \\
\text { Ilvesmäki, A. }\end{array}$ & $\begin{array}{l}\text { Finland, } \\
2012\end{array}$ & $\begin{array}{l}\text { Workplace Health } \\
\text { Promotion and } \\
\text { Stakeholder Positions: A } \\
\text { Finnish Case Study }\end{array}$ & ABCE & University & $\begin{array}{l}\text { Qualitative } \\
\text { approach, } \\
\text { Interviews, } \\
\text { thematic content } \\
\text { analysis }\end{array}$ \\
\hline 7 & $\begin{array}{l}\text { Nilsson, P. } \\
\text { Andersson, I.H. } \\
\text { Ejlertsson, G. } \\
\text { Troein, M. }\end{array}$ & $\begin{array}{l}\text { 2012, } \\
\text { Sweden }\end{array}$ & $\begin{array}{l}\text { Workplace health } \\
\text { resources based on sense } \\
\text { of coherence theory }\end{array}$ & ABCDE & University & $\begin{array}{l}\text { Salutogenic } \\
\text { approach, } \\
\text { focus group } \\
\text { interviews, } \\
\text { deductive analysis }\end{array}$ \\
\hline
\end{tabular}




\begin{tabular}{|c|c|c|c|c|c|c|}
\hline 8 & $\begin{array}{l}\text { Bauer, G.F. } \\
\text { Jenny, G. J. }\end{array}$ & $\begin{array}{l}\text { 2007, } \\
\text { Switzerland }\end{array}$ & $\begin{array}{l}\text { Development, } \\
\text { Implementation and } \\
\text { Dissemination of } \\
\text { Occupational Health } \\
\text { Management (OHM): } \\
\text { Putting salutogenesis into } \\
\text { practice }\end{array}$ & ABCDE & University & $\begin{array}{l}\text { preliminary } \\
\text { dissemination } \\
\text { study, the } \\
\text { longitudinal } \\
\text { reflection-group } \\
\text { design, quantitative } \\
\text { approach, } \\
\text { questionnaire }\end{array}$ \\
\hline 9 & $\begin{array}{l}\text { Grawitch, M. J. } \\
\text { Trares, S. } \\
\text { Kohler, J. M. }\end{array}$ & 2007, USA & $\begin{array}{l}\text { Healthy Workplace } \\
\text { Practices and Employee } \\
\text { Outcomes }\end{array}$ & $A B C E$ & University & $\begin{array}{l}\text { Exploratory } \\
\text { assessment, survey, } \\
\text { quantitative } \\
\text { approach and } \\
\text { analysis }\end{array}$ \\
\hline 10 & $\begin{array}{l}\text { Gregersen, S. } \\
\text { Vincent-Höper, S. } \\
\text { Nienhaus, A. }\end{array}$ & $\begin{array}{l}2014, \\
\text { Germany }\end{array}$ & $\begin{array}{l}\text { A comparison of } \\
\text { leadership constructs }\end{array}$ & $A B C E$ & University & $\begin{array}{l}\text { Quantitative } \\
\text { approach, } \\
\text { confirmatory factor } \\
\text { analysis approach, } \\
\text { descriptive } \\
\text { statistics and } \\
\text { correlations of } \\
\text { study variables }\end{array}$ \\
\hline 11 & $\begin{array}{l}\text { Martin, A. } \\
\text { Sanderson, K. } \\
\text { Scott, J. } \\
\text { Brough, P. }\end{array}$ & $\begin{array}{l}2009, \\
\text { Australia }\end{array}$ & $\begin{array}{l}\text { Promoting mental health } \\
\text { in small-medium } \\
\text { enterprises: An evaluation } \\
\text { of the "Business in Mind" } \\
\text { programme }\end{array}$ & $A B C E$ & University & $\begin{array}{l}\text { Quantitative } \\
\text { approach, cross- } \\
\text { sectional self-report } \\
\text { surveys, a three- } \\
\text { arm randomised } \\
\text { controlled trial }\end{array}$ \\
\hline 12 & Black, D. C. & UK, 2008 & $\begin{array}{l}\text { Working for a healthier } \\
\text { tomorrow }\end{array}$ & $\mathrm{BCE}$ & Institution & SLR \\
\hline 13 & $\begin{array}{l}\text { Briner, R. B. } \\
\text { Walshe, N. D. }\end{array}$ & USA, 2015, & $\begin{array}{l}\text { An evidence-based } \\
\text { approach to improving the } \\
\text { quality of resource- } \\
\text { oriented well-being } \\
\text { interventions at work }\end{array}$ & ABCE & University & $\begin{array}{l}\text { Evidence approach, } \\
\text { quantitative } \\
\text { approach, } \\
\text { questionnaires }\end{array}$ \\
\hline 14 & $\begin{array}{l}\text { Lewis, R. } \\
\text { Donaldson- } \\
\text { Feilder, E. } \\
\text { Jones, B. } \\
\text { Johal, M. }\end{array}$ & UK, 2014 & $\begin{array}{l}\text { Developing managers to } \\
\text { manage sustainable } \\
\text { employee engagement, } \\
\text { health and well-being }\end{array}$ & $A B C E$ & Institution & $\begin{array}{l}\text { Evidence based } \\
\text { practice approach, } \\
\text { interviews and } \\
\text { focus groups, } \\
\text { statistical analysis }\end{array}$ \\
\hline 15 & $\begin{array}{l}\text { Feldt, T. } \\
\text { Kinnunen, U. } \\
\text { Mauno, S. }\end{array}$ & $\begin{array}{l}\text { Finland, } \\
2000\end{array}$ & $\begin{array}{l}\text { A mediational model of } \\
\text { sense of coherence in the } \\
\text { work context: a one-year } \\
\text { follow-up study }\end{array}$ & ABCDE & University & $\begin{array}{l}\text { One-year follow-up } \\
\text { study, quantitative } \\
\text { approach, } \\
\text { questionnaires, } \\
\text { statistical analysis }\end{array}$ \\
\hline
\end{tabular}




\begin{tabular}{|c|c|c|c|c|c|c|}
\hline 16 & $\begin{array}{l}\text { Bringsén, A. } \\
\text { Andersson, H. I. } \\
\text { Ejlertsson, G. } \\
\text { Troein, M. }\end{array}$ & $\begin{array}{l}\text { Sweden, } \\
2012\end{array}$ & $\begin{array}{l}\text { Exploring workplace } \\
\text { related health resources } \\
\text { from a salutogenic } \\
\text { perspective: Results from } \\
\text { a focus group study } \\
\text { among healthcare } \\
\text { workers in Sweden }\end{array}$ & ABCDE & University & $\begin{array}{l}\text { Qualitative } \\
\text { approach, focus } \\
\text { group interviews, } \\
\text { qualitative content } \\
\text { analysis }\end{array}$ \\
\hline 17 & $\begin{array}{l}\text { Bauer, G.F. } \\
\text { Jenny, G. J. }\end{array}$ & $\begin{array}{l}\text { Switzerland, } \\
2012\end{array}$ & $\begin{array}{l}\text { Moving towards positive } \\
\text { organisational health: } \\
\text { challenges and a proposal } \\
\text { for a research model of } \\
\text { organisational health } \\
\text { development }\end{array}$ & $\mathrm{BCE}$ & University & SLR \\
\hline 18 & $\begin{array}{l}\text { Vogt, K. } \\
\text { Hakanen, J. J. } \\
\text { Jenny, G. J. } \\
\text { Bauer, G.F. }\end{array}$ & $\begin{array}{l}\text { Finland } \\
\text { Switzerland, } \\
2016,\end{array}$ & $\begin{array}{l}\text { Sense of Coherence and } \\
\text { the Motivational Process } \\
\text { of the Job-Demands- } \\
\text { Resources Model }\end{array}$ & ABCDE & University & $\begin{array}{l}\text { Longitudinal study, } \\
\text { quantitative } \\
\text { approach, } \\
\text { questionnaire, } \\
\text { statistical analysis }\end{array}$ \\
\hline 19 & Nilsson, $\mathrm{P}$. & $\begin{array}{l}\text { Sweden, } \\
2010\end{array}$ & $\begin{array}{l}\text { Enhance your workplace!: } \\
\text { a dialogue tool for } \\
\text { workplace health } \\
\text { promotion with } \\
\text { salutogenic approach }\end{array}$ & ABCDE & University & $\begin{array}{l}\text { Quantitative and } \\
\text { qualitative } \\
\text { approach, } \\
\text { questionnaires }\end{array}$ \\
\hline 20 & $\begin{array}{l}\text { Vinje, H. F. } \\
\text { Ausland, L.H. }\end{array}$ & $\begin{array}{l}\text { Sweden, } \\
2013\end{array}$ & $\begin{array}{l}\text { Salutogenic presence } \\
\text { supports a health- } \\
\text { promoting } \\
\text { work life }\end{array}$ & ABCDE & University & $\begin{array}{l}\text { Qualitative } \\
\text { approach, } \\
\text { explorative } \\
\text { design, interviews, } \\
\text { thematic analysis }\end{array}$ \\
\hline 21 & $\begin{array}{l}\text { Saksvik, P. } \varnothing . \\
\text { Olaniyan, O. S. } \\
\text { Lysklett, K. } \\
\text { Lien, M. } \\
\text { Bjerke, L. }\end{array}$ & $\begin{array}{l}\text { Norway, } \\
2015\end{array}$ & $\begin{array}{l}\text { A process evaluation of a } \\
\text { salutogenic intervention }\end{array}$ & ABCDE & University & $\begin{array}{l}\text { Quantitative and } \\
\text { qualitative } \\
\text { approach, } \\
\text { questionnaires, } \\
\text { interviews, } \\
\text { thematic analysis }\end{array}$ \\
\hline 22 & $\begin{array}{l}\text { Hauser, F. } \\
\text { Pleuger, F. } \\
\text { (in Fehlzeiten- } \\
\text { Report 2009) }\end{array}$ & $\begin{array}{l}\text { Germany, } \\
2010\end{array}$ & $\begin{array}{l}\text { Great Place to } \text { Work }^{\circledR} \text { : Ein } \\
\text { Arbeitsplatz, an dem man } \\
\text { sich wohl fühlt }\end{array}$ & ABCE & Institution & $\begin{array}{l}\text { Quantitative } \\
\text { approach, } \\
\text { questionnaires, } \\
\text { statistical analysis }\end{array}$ \\
\hline 23 & Friczewski, F. & $\begin{array}{l}\text { Germany, } \\
2006\end{array}$ & $\begin{array}{l}\text { Ein neues } \\
\text { Präventionsleitbild } \\
\text { psychischer Belastungen } \\
\text { am Arbeitsplatz }\end{array}$ & BCE & University & Not clear \\
\hline 24 & $\begin{array}{l}\text { Meyer, J. A. } \\
\text { (Study of }\end{array}$ & $\begin{array}{l}\text { Germany, } \\
2008\end{array}$ & Gesundheit in KMU & ABCE & Institution & $\begin{array}{l}\text { Quantitative } \\
\text { approach, }\end{array}$ \\
\hline
\end{tabular}




\begin{tabular}{|c|c|c|c|c|c|c|}
\hline & $\begin{array}{l}\text { Techniker } \\
\text { Krankenkasse } \\
(\mathrm{TK}))\end{array}$ & & & & & $\begin{array}{l}\text { questionnaires, } \\
\text { statistical analysis }\end{array}$ \\
\hline 25 & $\begin{array}{l}\text { Biallas, B. } \\
\text { Schäfe, D. } \\
\text { Dejonghe, L. } \\
\text { Franz, L. } \\
\text { Petrowski, K. } \\
\text { Froböse, I. } \\
\text { Wilk, C. }\end{array}$ & $\begin{array}{l}\text { Germany, } \\
2018\end{array}$ & $\begin{array}{l}\text { Präventionsreife in kleinen } \\
\text { und mittleren } \\
\text { Unternehmen }\end{array}$ & $A B C E$ & University & $\begin{array}{l}\text { Quantitative } \\
\text { approach, } \\
\text { questionnaires, } \\
\text { statistical analysis }\end{array}$ \\
\hline 26 & $\begin{array}{l}\text { Franke, F. } \\
\text { Felfe, J. }\end{array}$ & $\begin{array}{l}\text { Germany, } \\
2011\end{array}$ & $\begin{array}{l}\text { Diagnose gesundheits- } \\
\text { förderlicher Führung-Das } \\
\text { Instrument „Health- } \\
\text { oriented Leadership “ } \\
\text { (Fehlzeiten-Report 2011) }\end{array}$ & $\mathrm{ABCE}$ & University & $\begin{array}{l}\text { Qualitative } \\
\text { approach, } \\
\text { questionnaires }\end{array}$ \\
\hline 27 & Graeser, S. & $\begin{array}{l}\text { Germany, } \\
2011\end{array}$ & $\begin{array}{l}\text { Salutogenic factors for } \\
\text { mental health promotion } \\
\text { in work settings and } \\
\text { organizations }\end{array}$ & $A B C D$ & University & $\begin{array}{l}\text { Quantitative } \\
\text { approach, } \\
\text { questionnaires, } \\
\text { statistical analysis }\end{array}$ \\
\hline 28 & $\begin{array}{l}\text { Greasley, K. } \\
\text { Edwards, P. } \\
\text { Baker-McClearn, } \\
\text { D. } \\
\text { Dale, J. }\end{array}$ & UK, 2012 & $\begin{array}{l}\text { Why do organisations } \\
\text { engage in HR initiatives? A } \\
\text { test case of a health and } \\
\text { well-being intervention }\end{array}$ & $A B D$ & University & $\begin{array}{l}\text { Case study, } \\
\text { interviews and } \\
\text { observation }\end{array}$ \\
\hline 29 & $\begin{array}{l}\text { Gurt, J. } \\
\text { Schwennen, C. } \\
\text { Elke, G. }\end{array}$ & $\begin{array}{l}\text { Germany, } \\
2011\end{array}$ & $\begin{array}{l}\text { Health-specific leadership: } \\
\text { Is there an association } \\
\text { between leader } \\
\text { consideration for the } \\
\text { health of employees and } \\
\text { their strain and well- } \\
\text { being? }\end{array}$ & ABCE & University & $\begin{array}{l}\text { Quantitative } \\
\text { approach, } \\
\text { questionnaires, } \\
\text { statistical analysis }\end{array}$ \\
\hline 30 & $\begin{array}{l}\text { Henning, R. A. } \\
\text { Reeves, D. W. }\end{array}$ & USA, 2013 & $\begin{array}{l}\text { An integrated health } \\
\text { protection/promotion } \\
\text { programme supporting } \\
\text { participatory ergonomics } \\
\text { and salutogenic } \\
\text { approaches in the design } \\
\text { of workplace } \\
\text { interventions }\end{array}$ & ABCE & University & $\begin{array}{l}\text { Quantitative } \\
\text { approach, } \\
\text { questionnaires, } \\
\text { statistical analysis }\end{array}$ \\
\hline 31 & Nyberg, A. & $\begin{array}{l}\text { Sveden, } \\
2009\end{array}$ & $\begin{array}{l}\text { The impact of managerial } \\
\text { leadership on stress and } \\
\text { health among employees }\end{array}$ & $\mathrm{ABE}$ & University & $\begin{array}{l}\text { Quantitative } \\
\text { approach, } \\
\text { questionnaires, } \\
\text { statistical analysis }\end{array}$ \\
\hline
\end{tabular}




\begin{tabular}{|c|c|c|c|c|c|c|}
\hline 32 & $\begin{array}{l}\text { Vincent, S. } \\
\text { (in Fehlzeiten- } \\
\text { Report 2011) }\end{array}$ & $\begin{array}{l}\text { Germany, } \\
2011\end{array}$ & $\begin{array}{l}\text { Gesundheits-und } \\
\text { entwicklungsförderliches } \\
\text { Führungsverhalten: ein } \\
\text { Analyseinstrument }\end{array}$ & ABCE & University & $\begin{array}{l}\text { Quantitative } \\
\text { approach, } \\
\text { questionnaires, } \\
\text { statistical analysis }\end{array}$ \\
\hline 33 & $\begin{array}{l}\text { Vogt, K. } \\
\text { Jenny, G. J. } \\
\text { Bauer, G. F. }\end{array}$ & $\begin{array}{l}\text { Switzerland, } \\
2013\end{array}$ & $\begin{array}{l}\text { Comprehensibility, } \\
\text { manageability and } \\
\text { meaningfulness at work: } \\
\text { Construct validity of a } \\
\text { scale measuring work- } \\
\text { related sense of } \\
\text { coherence }\end{array}$ & ABCDE & University & $\begin{array}{l}\text { Quantitative } \\
\text { approach, } \\
\text { questionnaires, } \\
\text { statistical analysis }\end{array}$ \\
\hline 34 & $\begin{array}{l}\text { Ejlertsson, G. } \\
\text { Heijbel, B. } \\
\text { Andersson, H. I. }\end{array}$ & $\begin{array}{l}\text { Sweden, } \\
2018\end{array}$ & $\begin{array}{l}\text { Recovery, work-life } \\
\text { balance and work } \\
\text { experiences important to } \\
\text { self-rated health: A } \\
\text { questionnaire study on } \\
\text { salutogenic work factors } \\
\text { among Swedish primary } \\
\text { health care employees }\end{array}$ & $A B C D E$ & University & $\begin{array}{l}\text { Qualitative } \\
\text { approach, } \\
\text { questionnaires }\end{array}$ \\
\hline 35 & $\begin{array}{l}\text { Wollesen, B. } \\
\text { Menzel, J. } \\
\text { Drögemüller, R. } \\
\text { Hartwig, C. } \\
\text { Mattes, K. }\end{array}$ & $\begin{array}{l}\text { Germany, } \\
2016\end{array}$ & $\begin{array}{l}\text { The effects of a workplace } \\
\text { health promotion } \\
\text { program in small and } \\
\text { middle-sized companies: a } \\
\text { pre-post analysis }\end{array}$ & $A B C E$ & University & $\begin{array}{l}\text { Mixed method, } \\
\text { questionnaires and } \\
\text { qualitative } \\
\text { interviews, } \\
\text { statistical pre-post } \\
\text { analysis }\end{array}$ \\
\hline 36 & $\begin{array}{l}\text { Acas } \\
\text { (Advisory, } \\
\text { Conciliation and } \\
\text { Arbitration } \\
\text { Service) }\end{array}$ & UK, 2017 & $\begin{array}{l}\text { Addressing mental health } \\
\text { in the workplace by } \\
\text { creating a } \\
\text { culture of openness }\end{array}$ & $A B C E$ & Institution & $\begin{array}{l}\text { Qualitative } \\
\text { approach, } \\
\text { interviews }\end{array}$ \\
\hline 37 & $\begin{array}{l}\text { Acas } \\
\text { (Advisory, } \\
\text { Conciliation and } \\
\text { Arbitration } \\
\text { Service) }\end{array}$ & UK, 2017 & $\begin{array}{l}\text { Promoting positive mental } \\
\text { health at work by creating } \\
\text { a sense of shared } \\
\text { responsibility }\end{array}$ & ABCE & Institution & $\begin{array}{l}\text { Qualitative } \\
\text { approach, } \\
\text { interviews }\end{array}$ \\
\hline 38 & $\begin{array}{l}\text { CIPD } \\
\text { (Chartered } \\
\text { Institute of } \\
\text { Personnel and } \\
\text { Development) }\end{array}$ & UK, 2019 & $\begin{array}{l}\text { Health and well-being at } \\
\text { work }\end{array}$ & $A B C E$ & Institution & $\begin{array}{l}\text { Quantitative } \\
\text { approach, } \\
\text { questionnaires, } \\
\text { statistical analysis }\end{array}$ \\
\hline 39 & $\begin{array}{l}\text { INQA } \\
\text { (Die Initiative } \\
\text { Neue Qualität der } \\
\text { Arbeit) }\end{array}$ & $\begin{array}{l}\text { Germany, } \\
2017\end{array}$ & & ABCE & Institution & $\begin{array}{l}\text { Quantitative } \\
\text { approach, } \\
\text { questionnaires, } \\
\text { statistical analysis }\end{array}$ \\
\hline
\end{tabular}


An exploration of salutogenic factors promoting workforce health and well-being in small and medium-sized businesses in East-Westphalia Lippe/Germany

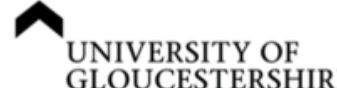

\begin{tabular}{|l|l|l|l|l|l|l|}
\hline 40 & INQA & Germany, & Führungskultur im Wandel & ABCE & Institution & Quantitative \\
(Die Initiative & 2014 & & & approach, \\
Neue Qualität der & & & & & questionnaires, \\
Arbeit) & & & & & statistical analysis \\
\hline
\end{tabular}




\subsection{Final studies with information after the first synthesis step}

\begin{tabular}{|c|c|c|c|c|}
\hline Author & $\begin{array}{l}\text { Place \& } \\
\text { time }\end{array}$ & Study & $\begin{array}{l}\text { Do the findings } \\
\text { show benefit of } \\
\text { WHP in terms of } \\
\text { salutogenesis? }\end{array}$ & Methodology \\
\hline $\begin{array}{l}\text { Nilsson, P. } \\
\text { Andersson, I.H. } \\
\text { Ejlertsson, G. } \\
\text { Troein, M. }\end{array}$ & $\begin{array}{l}\text { 2012, } \\
\text { Sweden }\end{array}$ & $\begin{array}{l}\text { Workplace health } \\
\text { resources based on } \\
\text { sense of coherence } \\
\text { theory }\end{array}$ & Yes & $\begin{array}{l}\text { Salutogenic approach, } \\
\text { focus group interviews, } \\
\text { deductive analysis }\end{array}$ \\
\hline $\begin{array}{l}\text { Bauer, G. } \\
\text { Jenny, G. }\end{array}$ & $\begin{array}{l}\text { 2007, } \\
\text { Switzerland }\end{array}$ & $\begin{array}{l}\text { Development, } \\
\text { Implementation and } \\
\text { Dissemination of } \\
\text { Occupational Health } \\
\text { Management } \\
\text { (OHM): } \\
\text { Putting } \\
\text { salutogenesis into } \\
\text { practice }\end{array}$ & Yes & $\begin{array}{l}\text { preliminary } \\
\text { dissemination study, the } \\
\text { longitudinal reflection- } \\
\text { group design, } \\
\text { quantitative approach, } \\
\text { questionnaire }\end{array}$ \\
\hline $\begin{array}{l}\text { Feldt, T. } \\
\text { Kinnunen, U. } \\
\text { Mauno, S. }\end{array}$ & $\begin{array}{l}\text { Finland, } \\
2000\end{array}$ & $\begin{array}{l}\text { A mediational } \\
\text { model of sense of } \\
\text { coherence in the } \\
\text { work context: a } \\
\text { one-year follow-up } \\
\text { study }\end{array}$ & Yes & $\begin{array}{l}\text { One-year follow-up } \\
\text { study, quantitative } \\
\text { approach, } \\
\text { questionnaires, } \\
\text { statistical analysis }\end{array}$ \\
\hline $\begin{array}{l}\text { Bringsén, A. } \\
\text { Andersson, H. I. } \\
\text { Ejlertsson, G. } \\
\text { Troein, M. }\end{array}$ & $\begin{array}{l}\text { Sweden, } \\
2012\end{array}$ & $\begin{array}{l}\text { Exploring workplace } \\
\text { related health } \\
\text { resources from a } \\
\text { salutogenic } \\
\text { perspective: Results } \\
\text { from a focus group } \\
\text { study among } \\
\text { healthcare } \\
\text { workers in Sweden }\end{array}$ & yes & $\begin{array}{l}\text { Qualitative approach, } \\
\text { focus group interviews, } \\
\text { qualitative content } \\
\text { analysis }\end{array}$ \\
\hline $\begin{array}{l}\text { Vogt, K. } \\
\text { Hakanen, J. J. } \\
\text { Jenny, G. J. } \\
\text { Bauer, G.F. }\end{array}$ & $\begin{array}{l}\text { Finland } \\
\text { Switzerland } \\
, 2016\end{array}$ & $\begin{array}{l}\text { Sense of Coherence } \\
\text { and the } \\
\text { Motivational } \\
\text { Process of the Job- } \\
\text { Demands- } \\
\text { Resources Model }\end{array}$ & Yes & $\begin{array}{l}\text { Longitudinal study, } \\
\text { quantitative approach, } \\
\text { questionnaire, statistical } \\
\text { analysis }\end{array}$ \\
\hline Nilsson, $\mathrm{P}$. & $\begin{array}{l}\text { Sweden, } \\
2010\end{array}$ & $\begin{array}{l}\text { Enhance your } \\
\text { workplace!: a } \\
\text { dialogue tool for } \\
\text { workplace health }\end{array}$ & Yes & $\begin{array}{l}\text { Quantitative and } \\
\text { qualitative approach, } \\
\text { questionnaires }\end{array}$ \\
\hline
\end{tabular}


An exploration of salutogenic factors promoting workforce health and well-being in small and medium-sized businesses in East-Westphalia Lippe/Germany

\begin{tabular}{|c|c|c|c|c|}
\hline & & $\begin{array}{l}\text { promotion with } \\
\text { salutogenic } \\
\text { approach }\end{array}$ & & \\
\hline $\begin{array}{l}\text { Vinje, H. F. } \\
\text { Ausland, L.H. }\end{array}$ & $\begin{array}{l}\text { Sweden, } \\
2013\end{array}$ & $\begin{array}{l}\text { Salutogenic } \\
\text { presence supports a } \\
\text { health promoting } \\
\text { work life }\end{array}$ & & $\begin{array}{l}\text { Qualitative approach, } \\
\text { explorative } \\
\text { design, interviews, } \\
\text { thematic analysis }\end{array}$ \\
\hline $\begin{array}{l}\text { Saksvik, P. Ø. } \\
\text { Olaniyan, O. S. } \\
\text { Lysklett, K. } \\
\text { Lien, M. } \\
\text { Bjerke, L. }\end{array}$ & $\begin{array}{l}\text { Norway, } \\
2015\end{array}$ & $\begin{array}{l}\text { A process } \\
\text { evaluation of a } \\
\text { salutogenic } \\
\text { intervention }\end{array}$ & Yes & $\begin{array}{l}\text { Quantitative and } \\
\text { qualitative approach, } \\
\text { questionnaires, } \\
\text { interviews, thematic } \\
\text { analysis }\end{array}$ \\
\hline $\begin{array}{l}\text { Vogt, K. } \\
\text { Jenny, G. J. } \\
\text { Bauer, G. F. }\end{array}$ & $\begin{array}{l}\text { Switzerland } \\
, 2013\end{array}$ & $\begin{array}{l}\text { Comprehensibility, } \\
\text { manageability and } \\
\text { meaningfulness at } \\
\text { work: Construct } \\
\text { validity of a scale } \\
\text { measuring work- } \\
\text { related sense of } \\
\text { coherence }\end{array}$ & Yes & $\begin{array}{l}\text { Quantitative approach, } \\
\text { questionnaires, } \\
\text { statistical analysis }\end{array}$ \\
\hline $\begin{array}{l}\text { Ejlertsson, G. } \\
\text { Heijbel, B. } \\
\text { Andersson, H. I. }\end{array}$ & $\begin{array}{l}\text { Sweden, } \\
2018\end{array}$ & $\begin{array}{l}\text { Recovery, work-life } \\
\text { balance and work } \\
\text { experiences } \\
\text { important to self- } \\
\text { rated health: A } \\
\text { questionnaire study } \\
\text { on salutogenic work } \\
\text { factors among } \\
\text { Swedish primary } \\
\text { health care } \\
\text { employees }\end{array}$ & Yes & $\begin{array}{l}\text { Qualitative approach, } \\
\text { questionnaires }\end{array}$ \\
\hline
\end{tabular}




\subsection{Final studies with information after the second synthesis step}

\begin{tabular}{|c|c|c|c|c|c|}
\hline Author & Study & $\begin{array}{l}\text { Do the } \\
\text { findings } \\
\text { show...? }\end{array}$ & $\begin{array}{l}\text { Organisation } \\
\& \\
\text { participants }\end{array}$ & Intervention & $\begin{array}{l}\text { Significant factors } \\
\text { related to } \\
\text { implementation }\end{array}$ \\
\hline $\begin{array}{l}\text { Nilsson, P. } \\
\text { Andersson, } \\
\text { I.H. } \\
\text { Ejlertsson, } \\
\text { G. } \\
\text { Troein, M. }\end{array}$ & $\begin{array}{l}\text { Workplace } \\
\text { health } \\
\text { resources based } \\
\text { on sense of } \\
\text { coherence } \\
\text { theory }\end{array}$ & Yes & $\begin{array}{l}1 \text { Hospital, } \\
\text { employees }\end{array}$ & $\begin{array}{l}\text { A communication tool } \\
\text { called SER may serve as } \\
\text { initial examples in } \\
\text { workplace discussions } \\
\text { about work-related } \\
\text { resources contributing } \\
\text { to a sense of coherence } \\
\text { related to } \\
\text { implementation }\end{array}$ & $\begin{array}{l}\text { Opportunity of SER to } \\
\text { explore, understand and } \\
\text { foster workplace } \\
\text { resources }\end{array}$ \\
\hline $\begin{array}{l}\text { Bauer, G. } \\
\text { Jenny, G. }\end{array}$ & $\begin{array}{l}\text { Development, } \\
\text { Implementation } \\
\text { and } \\
\text { Dissemination } \\
\text { of Occupational } \\
\text { Health } \\
\text { Management } \\
\text { (OHM): } \\
\text { Putting } \\
\text { salutogenesis } \\
\text { into practice }\end{array}$ & Yes & $\begin{array}{l}10 \text { SMEs, } \\
\text { employees } \\
\text { (Human } \\
\text { resource } \\
\text { manager) }\end{array}$ & $\begin{array}{l}\text { A systemic-salutogenic } \\
\text { model of OHM which } \\
\text { emphasises the } \\
\text { salutogenic perspective: } \\
\text { concentrating on } \\
\text { salutogenic factors and } \\
\text { practices and how to } \\
\text { optimise them } \\
\text { sustainably }\end{array}$ & $\begin{array}{l}\text { Study propose to focus } \\
\text { on three phases of OHM } \\
\text { development in a } \\
\text { company: a pre- } \\
\text { intentional, } \\
\text { contemplation phase, a } \\
\text { preactional, preparation } \\
\text { phase, and an actional } \\
\text { phase. }\end{array}$ \\
\hline $\begin{array}{l}\text { Feldt, T. } \\
\text { Kinnunen, } \\
\text { U. } \\
\text { Mauno, S. }\end{array}$ & $\begin{array}{l}\text { A mediational } \\
\text { model of sense } \\
\text { of coherence in } \\
\text { the work } \\
\text { context: a one- } \\
\text { year follow-up } \\
\text { study }\end{array}$ & Yes & $\begin{array}{l}4 \\
\text { Organisations } \\
219 \\
\text { employees }\end{array}$ & $\begin{array}{l}\text { Testing of a mediational } \\
\text { model appropriate for } \\
\text { explaining the effects of } \\
\text { psychosocial work } \\
\text { characteristics on well- } \\
\text { being }\end{array}$ & $\begin{array}{l}\text { major role of a good } \\
\text { organisational climate } \\
\text { as a factor enhancing } \\
\text { SOC, and consequently } \\
\text { well-being. }\end{array}$ \\
\hline $\begin{array}{l}\text { Bringsén, } \\
\text { A. } \\
\text { Andersson, } \\
\text { H. I. } \\
\text { Ejlertsson, } \\
\text { G. } \\
\text { Troein, M. }\end{array}$ & $\begin{array}{l}\text { Exploring } \\
\text { workplace } \\
\text { related health } \\
\text { resources from } \\
\text { a salutogenic } \\
\text { perspective: } \\
\text { Results from a } \\
\text { focus group } \\
\text { study among } \\
\text { healthcare } \\
\text { workers in } \\
\text { Sweden }\end{array}$ & yes & $\begin{array}{l}1 \text { Hospital, } \\
35 \text { nurses/ } \\
\text { assistant } \\
\text { nurses }\end{array}$ & $\begin{array}{l}\text { Identify patterns in the } \\
\text { expressed opinions of } \\
\text { the participants } \\
\text { regarding health } \\
\text { resources }\end{array}$ & $\begin{array}{l}\text { two divergent patterns; } \\
\text { some of the participants } \\
\text { associated positive } \\
\text { health with stability } \\
\text { while others referred to } \\
\text { flexibility. The identified } \\
\text { pattern of diversity } \\
\text { highlights the } \\
\text { importance of always } \\
\text { exploring the needs of } \\
\text { the particular workers } \\
\text { at hand, in order to }\end{array}$ \\
\hline
\end{tabular}




\begin{tabular}{|c|c|c|c|c|c|}
\hline & & & & & $\begin{array}{l}\text { facilitate positive effects } \\
\text { from WHP initiatives. }\end{array}$ \\
\hline $\begin{array}{l}\text { Vogt, K. } \\
\text { Hakanen, } \\
\text { J. J. } \\
\text { Jenny, G. J. } \\
\text { Bauer, G.F. }\end{array}$ & $\begin{array}{l}\text { Sense of } \\
\text { Coherence } \\
\text { and the } \\
\text { Motivational } \\
\text { Process of the } \\
\text { Job-Demands- } \\
\text { Resources } \\
\text { Model }\end{array}$ & Yes & $\begin{array}{l}\text { Broad range } \\
\text { of } \\
\text { enterprises, } \\
950 \\
\text { employees }\end{array}$ & $\begin{array}{l}\text { Examination of the } \\
\text { various roles played by } \\
\text { the personal resource } \\
\text { SoC in the motivational } \\
\text { process described by the } \\
\text { job-demands-resources } \\
\text { model }\end{array}$ & $\begin{array}{l}\text { The findings confirm } \\
\text { that providing a } \\
\text { resourceful working } \\
\text { environment can help } \\
\text { to build employee SoC } \\
\text { and the creation of } \\
\text { additional job } \\
\text { resources, leading to } \\
\text { positive outcomes such } \\
\text { as work } \\
\text { engagement. }\end{array}$ \\
\hline Nilsson, P. & $\begin{array}{l}\text { Enhance your } \\
\text { workplace! a } \\
\text { dialogue tool } \\
\text { for workplace } \\
\text { health } \\
\text { promotion with } \\
\text { salutogenic } \\
\text { approach }\end{array}$ & Yes & $\begin{array}{l}\text { Hospitals, } \\
220 \\
\text { employees }\end{array}$ & $\begin{array}{l}\text { Identifying detailed } \\
\text { salutogenic workplace } \\
\text { related factors and } \\
\text { processes among } \\
\text { hospital staff with the } \\
\text { SOC theory as a } \\
\text { framework via WEMS } \\
\text { (The Work } \\
\text { Experience } \\
\text { Measurement Scale) }\end{array}$ & $\begin{array}{l}\text { The WEMS } \\
\text { questionnaire is a } \\
\text { contribution to research } \\
\text { about workplace- } \\
\text { related experiences } \\
\text { from a salutogenic } \\
\text { perspective. In addition, } \\
\text { it is useful for } \\
\text { investigating work- } \\
\text { related experiences by } \\
\text { people of different } \\
\text { professions and ages. }\end{array}$ \\
\hline $\begin{array}{l}\text { Vinje, H. F., } \\
\text { Ausland, } \\
\text { L.H. }\end{array}$ & $\begin{array}{l}\text { Salutogenic } \\
\text { presence } \\
\text { supports a } \\
\text { health } \\
\text { promoting } \\
\text { work life }\end{array}$ & Yes & $\begin{array}{l}1 \\
\text { Organisation } \\
\text { in municipal } \\
\text { health and } \\
\text { care sector, } \\
\text { human } \\
\text { resources } \\
\text { manager and } \\
\text { chief } \\
\text { municipal } \\
\text { health and } \\
\text { social welfare } \\
\text { Officer }\end{array}$ & $\begin{array}{l}\text { Salutogenic presence as } \\
\text { experienced by senior } \\
\text { leaders and co-workers } \\
\text { in the municipal health } \\
\text { and care sector. }\end{array}$ & $\begin{array}{l}\text { Workplace itself can } \\
\text { contribute to building } \\
\text { health-promoting } \\
\text { working life by devoting } \\
\text { attention to salutogenic } \\
\text { presence. That can be } \\
\text { done by seeking out and } \\
\text { paving the way for } \\
\text { opportunities to explore } \\
\text { the qualities of } \\
\text { presence and the } \\
\text { dynamics of health in } \\
\text { the workplace, both } \\
\text { individually and in } \\
\text { groups. }\end{array}$ \\
\hline $\begin{array}{l}\text { Saksvik, P. } \\
\varnothing . \\
\text { Olaniyan, }\end{array}$ & $\begin{array}{l}\text { A process } \\
\text { evaluation of a } \\
\text { salutogenic }\end{array}$ & Yes & $\begin{array}{l}1 \text { Consultant } \\
\text { company, } \\
\text { employees }\end{array}$ & $\begin{array}{l}\text { Evaluation of an } \\
\text { intervention labelled } \\
\text { 'the employeeship- }\end{array}$ & $\begin{array}{l}\text { More attention should } \\
\text { be centred on } \\
\text { developing and }\end{array}$ \\
\hline
\end{tabular}




\begin{tabular}{|c|c|c|c|c|c|}
\hline $\begin{array}{l}\text { O. S. } \\
\text { Lysklett, K. } \\
\text { Lien, M. } \\
\text { Bjerke, L. }\end{array}$ & intervention & & & $\begin{array}{l}\text { programme' } \\
\text { Strengthening the } \\
\text { psychosocial work } \\
\text { environment by raising } \\
\text { the employees' } \\
\text { awareness of and } \\
\text { competence in } \\
\text { interpersonal } \\
\text { relationships }\end{array}$ & $\begin{array}{l}\text { increasing positive } \\
\text { psychosocial } \\
\text { experiences, while } \\
\text { simultaneously } \\
\text { indirectly reducing the } \\
\text { negative. This has now } \\
\text { become a new trend in } \\
\text { the traditional approach } \\
\text { as well; to reduce stress } \\
\text { and improve the mental } \\
\text { and physical health of } \\
\text { employees by } \\
\text { introducing positive } \\
\text { change initiatives }\end{array}$ \\
\hline $\begin{array}{l}\text { Vogt, K. } \\
\text { Jenny, G. J. } \\
\text { Bauer, G. F. }\end{array}$ & $\begin{array}{l}\text { Comprehensibili } \\
\text { ty, } \\
\text { manageability } \\
\text { and } \\
\text { meaningfulness } \\
\text { at work: } \\
\text { Construct } \\
\text { validity of a } \\
\text { scale measuring } \\
\text { work-related } \\
\text { sense of } \\
\text { coherence }\end{array}$ & Yes & $\begin{array}{l}8 \text { enterprises } \\
\text { (medium to } \\
\text { large), } \\
\text { diverse } \\
\text { economic } \\
\text { sectors (four } \\
\text { industrial- } \\
\text { production } \\
\text { companies, } \\
\text { one food- } \\
\text { processing } \\
\text { company, } \\
\text { one public- } \\
\text { administratio } \\
\text { n service and } \\
\text { two } \\
\text { hospitals). } \\
\text { employees }\end{array}$ & $\begin{array}{l}\text { Investigate the factorial } \\
\text { invariance and the } \\
\text { construct validity of a } \\
\text { scale that measures } \\
\text { Work-SoC. }\end{array}$ & $\begin{array}{l}\text { Work-SoC might serve } \\
\text { as a practical screening } \\
\text { instrument for assessing } \\
\text { an employee's } \\
\text { perception of the } \\
\text { potential health- } \\
\text { promoting qualities of } \\
\text { his or her current work } \\
\text { situation. In line with a } \\
\text { call for positive health, } \\
\text { the construct of Work- } \\
\text { SoC emphasises a } \\
\text { resource-oriented view } \\
\text { on workplace health } \\
\text { promotion. The study } \\
\text { advances both the } \\
\text { salutogenic theory and } \\
\text { the field of positive } \\
\text { occupational health } \\
\text { psychology by } \\
\text { redefining sense of } \\
\text { coherence as an } \\
\text { interactional and } \\
\text { context-specific } \\
\text { fonstruct that is useful } \\
\text { resvention }\end{array}$ \\
\hline $\begin{array}{l}\text { Ejlertsson, } \\
\text { G. }\end{array}$ & $\begin{array}{l}\text { Recovery, work- } \\
\text { life balance and }\end{array}$ & Yes & $\begin{array}{l}26 \text { primary } \\
\text { health care }\end{array}$ & $\begin{array}{l}\text { A questionnaire study } \\
\text { on salutogenic work }\end{array}$ & $\begin{array}{l}3 \text { areas of special } \\
\text { importance: recovery, }\end{array}$ \\
\hline
\end{tabular}


An exploration of salutogenic factors promoting workforce health and well-being in small and medium-sized businesses in East-Westphalia Lippe/Germany

UNIVERSITY OF GLOUCESTERSHIRE

\begin{tabular}{|c|c|c|c|c|}
\hline $\begin{array}{l}\text { Heijbel, B. } \\
\text { Andersson, } \\
\text { H. I. }\end{array}$ & $\begin{array}{l}\text { work } \\
\text { experiences } \\
\text { important to } \\
\text { self-rated } \\
\text { health: A } \\
\text { questionnaire } \\
\text { study on } \\
\text { salutogenic } \\
\text { work factors } \\
\text { among Swedish } \\
\text { primary health } \\
\text { care employees }\end{array}$ & $\begin{array}{l}\text { centres, } 599 \\
\text { health care } \\
\text { employees }\end{array}$ & $\begin{array}{l}\text { factors among Swedish } \\
\text { primary health care } \\
\text { employees }\end{array}$ & $\begin{array}{l}\text { work-life balance and } \\
\text { work experiences. } \\
\text { Recovery during work } \\
\text { was shown to be of } \\
\text { great importance. }\end{array}$ \\
\hline
\end{tabular}




\subsection{Example of code table}

\begin{tabular}{|c|c|c|}
\hline \multirow{10}{*}{ 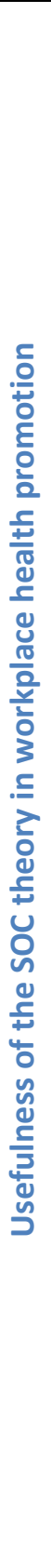 } & $\begin{array}{l}\text { Nilsson, P. } \\
\text { Andersson, I.H. } \\
\text { Ejlertsson, G. } \\
\text { Troein, M. }\end{array}$ & $\begin{array}{l}\text { Exploration of the usability of the sense of coherence (SOC) theory to identify } \\
\text { considerable and positively perceived work-related factors and processes }\end{array}$ \\
\hline & $\begin{array}{l}\text { Bauer, G. } \\
\text { Jenny, G. }\end{array}$ & Advocate salutogenesis as the theoretical basis for this OHM approach \\
\hline & $\begin{array}{l}\text { Feldt, T. } \\
\text { Kinnunen, U. } \\
\text { Mauno, S. }\end{array}$ & $\begin{array}{l}\text { Testing a mediational model appropriate for explaining the aspects of psychosocial work } \\
\text { characteristics on general well-being, and on occupational well-being via sense of } \\
\text { coherence (SOC) }\end{array}$ \\
\hline & $\begin{array}{l}\text { Bringsén, A. } \\
\text { Andersson, H. I. } \\
\text { Ejlertsson, G. } \\
\text { Troein, M. }\end{array}$ & $\begin{array}{l}\text { Exploring workplace related health resources from a salutogenic perspective. The results } \\
\text { from this study have contributed to the body of knowledge regarding salutogenic health } \\
\text { indicators in the field of work }\end{array}$ \\
\hline & $\begin{array}{l}\text { Vogt, K. } \\
\text { Hakanen, J. J. } \\
\text { Jenny, G. J. } \\
\text { Bauer, G.F. }\end{array}$ & $\begin{array}{l}\text { Examination of various roles played by the personal resource "sense of coherence" (SoC) in } \\
\text { the motivational process described by the job-demands-resources model }\end{array}$ \\
\hline & Nilsson, $\mathrm{P}$. & $\begin{array}{l}\text { Developing a tool, including a questionnaire and a dialogue process, which can be used for } \\
\text { workplace health promotion from a salutogenic point of view }\end{array}$ \\
\hline & $\begin{array}{l}\text { Vinje, H. F., } \\
\text { Ausland, L.H. }\end{array}$ & Experiencing and exploring the characteristics of presence with a salutogenic view \\
\hline & $\begin{array}{l}\text { Saksvik, P. } \varnothing . \\
\text { Olaniyan, O. S. } \\
\text { Lysklett, K. } \\
\text { Lien, M. } \\
\text { Bjerke, L. }\end{array}$ & $\begin{array}{l}\text { Evaluation of a mandatory salutogenic intervention to learn more about the contributions of } \\
\text { these kinds of interventions to health-related outcomes and of the implementation of the } \\
\text { intervention }\end{array}$ \\
\hline & $\begin{array}{l}\text { Vogt, K. } \\
\text { Jenny, G. J. } \\
\text { Bauer, G. F. }\end{array}$ & $\begin{array}{l}\text { Investigation of factorial invariance and the construct validity of a scale that measures } \\
\text { Work-SoC }\end{array}$ \\
\hline & $\begin{array}{l}\text { Ejlertsson, G. } \\
\text { Heijbel, B. } \\
\text { Andersson, H. I. }\end{array}$ & Exploration of salutogenic work-related factors among primary health care employees \\
\hline
\end{tabular}




\subsection{Grading system assessing the robustness of the synthesis.}

\begin{tabular}{|c|c|c|c|c|}
\hline Final studies & $\begin{array}{l}\text { Relevance regarding } \\
\text { review research question }\end{array}$ & $\begin{array}{l}\text { Relevance regarding } \\
\text { study research questions }\end{array}$ & $\begin{array}{l}\text { Validity shown by } \\
\text { methodological } \\
\text { soundness }\end{array}$ & $\begin{array}{l}\text { Appropriateness of } \\
\text { study design } \\
\text { (relevance regarding } \\
\text { research objectives) }\end{array}$ \\
\hline $\begin{array}{l}\text { Nilsson, P. } \\
\text { Andersson, I.H. } \\
\text { Ejlertsson, G. } \\
\text { Troein, M. }\end{array}$ & $\checkmark$ & $\checkmark$ & $\checkmark$ & $\checkmark$ \\
\hline $\begin{array}{l}\text { Bauer, G.F } \\
\text { Jenny, G. J. }\end{array}$ & $\checkmark$ & $\checkmark$ & $\checkmark$ & $X$ \\
\hline $\begin{array}{l}\text { Feldt, T. } \\
\text { Kinnunen, U. } \\
\text { Mauno, S. }\end{array}$ & $\checkmark$ & $\checkmark$ & $\checkmark$ & $\checkmark$ \\
\hline $\begin{array}{l}\text { Bringsén, A. } \\
\text { Andersson, H. I. } \\
\text { Ejlertsson, G. } \\
\text { Troein, M. }\end{array}$ & $\checkmark$ & $\checkmark$ & $\checkmark$ & $\checkmark$ \\
\hline $\begin{array}{l}\text { Vogt, K. } \\
\text { Hakanen, J. J. } \\
\text { Jenny, G. J. } \\
\text { Bauer, G.F. }\end{array}$ & $\checkmark$ & $\checkmark$ & $\checkmark$ & $\checkmark$ \\
\hline Nilsson, $\mathrm{P}$. & $\checkmark$ & $\checkmark$ & $\checkmark$ & $\checkmark$ \\
\hline $\begin{array}{l}\text { Vinje, H. F., } \\
\text { Ausland, L.H. }\end{array}$ & $\checkmark$ & $\checkmark$ & $\checkmark$ & $\checkmark$ \\
\hline $\begin{array}{l}\text { Saksvik, P. Ø. } \\
\text { Olaniyan, O. S. } \\
\text { Lysklett, K. } \\
\text { Lien, M. } \\
\text { Bjerke, L. }\end{array}$ & $\checkmark$ & $\checkmark$ & $\checkmark$ & $\checkmark$ \\
\hline $\begin{array}{l}\text { Vogt, K. } \\
\text { Jenny, G. J. } \\
\text { Bauer, G. F. }\end{array}$ & $\checkmark$ & $\checkmark$ & $\checkmark$ & $X$ \\
\hline $\begin{array}{l}\text { Ejlertsson, G. } \\
\text { Heijbel, B. } \\
\text { Andersson, H. I. }\end{array}$ & $\checkmark$ & $\checkmark$ & $\checkmark$ & $\checkmark$ \\
\hline
\end{tabular}




\section{Einverständniserklärung Gruppendiskussion}

Bei der Gruppendiskussion, an der Sie teilnehmen werden, werden Sie unter anderem nach Ihrer persönlichen Meinung zu Maßnahmen der Gesundheitsförderung von Mitarbeitern/innen befragt und dazu aufgefordert, mit anderen Teilnehmern/innen darüber zu diskutieren. Die Gruppendiskussion dient zur Erkenntnisgewinnung, welche Maßnahmen/Faktoren die Gesundheit und das Wohlbefinden von Mitarbeitern fördern und durch Unternehmer in Ostwestfalen Lippe erzeugt werden.

\section{Ablauf der Gruppendiskussion}

Als Teilnehmer der Gruppendiskussion bitten wir Sie darum,

- offen gegenüber Neuerungen der Gesundheitsförderung zu sein

- mit anderen Teilnehmern darüber zu diskutieren (Vorteile/Nachteile).

\section{Hinweis}

Sie dürfen die Gruppendiskussion zu jedem beliebigen Zeitpunkt verlassen.

\section{Weiterverwendung der Daten aus der Gruppendiskussion}

Der Verlauf wird digital aufgezeichnet (Audio). Alle Daten werden anonymisiert eingesehen und ausgewertet.

\section{Verschwiegenheitserklärung der Teilnehmer/innen}

Hiermit weise ich auf die Vertraulichkeit der Gruppendiskussion und ihre Verschwiegenheitspflicht hin. Mit Ihrer Unterschrift verpflichten Sie sich, keine Details aus der Gruppendiskussion an Dritte weiterzugeben.

Ich habe die obigc Einverständniserklärung zur Gruppendiskussion, zur Verwendung der Daten und die Verschwiegenheitserklärung gelesen und erklăre mich mit allen Punkten einverstanden.

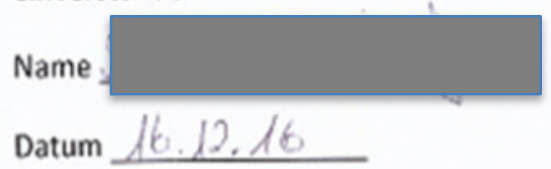

Unternehmen

Unterschrift 


\subsection{Codebook}

Code: 1 Boss Pion Ini

Brief Definition: Boss as pioneer and initiator

Definition \& representation of the scenario (opinion of Mr. Müller): I am trendsetter in the matter of health promotion. I pay attention to my own health. I would like to influence others to promote leading also healthy lives. I recognize early warning signals from my employees and react to them. Question within focus-group guideline: Q1. Mr. Müller is a pioneer as a boss: How is that with you in your enterprise? How can this be implemented? What are your experiences?

RQ 1 \& 3: Contributes to define salutogenic factors that can be generated by top management to support the promotion of health and well-being of workforce and contributes to understand how SMEs in EWL/Germany integrate health promoting and well-being factors.

Example: „So assuming a certain role model in the subject of health is certainly advantageous in my opinion [...]."

\section{Code: 2 Bre}

Brief Definition: Break

Definition \& representation of the scenario (opinion of Mr. Müller): I take care of my health. I don't go to work when I am sick. I take a nap in my lunch break. There are times when I don't make appointments. I rarely work overtime, so I have time for my family.

Question within focus-group guideline: Q2. Mr. Müller takes care of his own health: How is that with you in your enterprise? How can this be implemented? What are your experiences?

RQ 1 \& 3: Contributes to define salutogenic factors that can be generated by top management to support the promotion of health and well-beingof workforce and contributes to understand how SMEs in EWL/Germany integrate health promoting and well-being factors.

Example: „Of course everyone can use his lunch break as he likes. The one smokes, the other sleeps. And when you have to provide beds, a dormitory, because the demand is there, I think we would not mind."

\section{Code: 3 Appre praise}

Brief Definition: Appreciation by praise

Definition \& representation of the scenario (opinion of Mr. Müller): I value my employees' worth: As I and my managers praise the employees when they have done a good job. By constantly adapting our bonuses to current trends (wellness and spa flat rate for half a year etc.). By 
distributing praise cards when employees have done something remarkable. By enabling the employees to attend a department of their choice one week a year.

Question within focus-group guideline: Q5. Mr. Müller values his employees' worth: How is that with you in your enterprise? How can this be implemented? What are your experiences?

RQ 1 \& 3: Contributes to define salutogenic factors that can be generated by top management to support the promotion of health and well-being of workforce and contributes to understand how SMEs in EWL/Germany integrate health promoting and well-being factors.

Example: „So you don't need a star in the class book [...], but just a normal thanks. That would be nice."

\section{Code: 4 Pos areas cont}

Brief Definition: Possibility to learn about other areas and to place the own work into the bigger context

Definition \& representation of the scenario (opinion of Mr. Müller): I create for my employees a meaning in the work: As everyone knows what he is doing to achieve company goals. By explaining what the work of the individual has to do with the final product. By communicating my vision of my enterprise and explaining to the employees their contribution to it. By explaining the contribution of the product to the larger whole.

Question within focus-group guideline: Q4. Mr. Müller creates a meaning in the work for his employees: How is that with you in your enterprise? How can this be implemented? What are your experiences?

RQ 1 \& 3: Contributes to define salutogenic factors that can be generated by top management to support the promotion of health and well-being of workforce and contributes to understand how SMEs in EWL/Germany integrate health promoting and well-being factors.

Example: „When I started with the X company, I decided to get to know the company, and then I was given the opportunity to be in the company for a week, because it was very important for me to get to know all the processes , which of course helps me a lot in my daily work."

\section{Code: 5 Self resp empl}

Brief Definition: Self-responsibility of employees

Definition \& representation of the scenario (opinion of Mr. Müller): I promote the independence of my employees, but I am also a safety -net at the same time, if they need me. I am prepared to let go of important issues and delegate them. I leave my employees a great deal of selfdetermination. I assure them of my support if they do not move on their own. I say "no" to customers, if it is necessary for the health/performance of my employees. 
Question within focus-group guideline: Q3. Mr. Müller promotes the independence of his employees and he is also a safety -net at the same time: How is that with you in your enterprise? How can this be implemented? What are your experiences?

RQ 2 \& 3: Contributes to develop top management awareness and contributes to understand how SMEs in EWL/Germany integrate health promoting and well-being factors.

Example: „Self-responsibility, there are two things in the concept (word) which begins with self."

Code: 6 Coop cult

Brief Definition: Corporate culture

Definition \& representation of the scenario (opinion of Mr. Müller): I encourage my employees: By making sure that the work content matches the skills of the employees. By distributing tasks so that the resources of the employees are in harmony with the level of skill and abilities. By distributing tasks so that the resources of the employees are in harmony with the level of skill and abilities. By making sure that the employees' development opportunities become clear.

Question within focus-group guideline: Q6. Mr. Müller encourages his employees: How is that with you in your enterprise? How can this be implemented? What are your experiences?

RQ 1 \& 3: Contributes to define salutogenic factors that can be generated by top management to support the promotion of health and well-being of workforce and contributes to understand how SMEs in EWL/Germany integrate health promoting and well-being factors.

Example: „Company culture is always strongly influenced by the top manager, through them personally, through their top manager family, but as a corporate culture, I tell all employees, we are all parts of a building block, with influence, so this DNA is naturally also belonging to the top manager, the top manager family, I must already say. The subject of praise or feedback I would say is a very, very important issue."

\section{Code: 7 Sen together}

Brief Definition: SMEs strengthen the sense of togetherness

Definition \& representation of the scenario (opinion of Mr. Müller): I would like to continually expand and improve the health promotion of my employees. Are there any other ideas? Question within focus-group guideline: Q10. Mr. Müller would like to continually expand and improve the health promotion of his employees. Are there any other ideas?

RQ 1 \& 3: Contributes to define salutogenic factors that can be generated by top management to support the promotion of health and well-being of workforce and contributes to understand how SMEs in EWL/Germany integrate health promoting and well-being factors.

Example: „But what we have found is that we have a better athmosphere in the company when 
we do what we do on a regular basis. [...]. And then we're on the theme 'movement', because if we do something, we are doing something active, we go cycling, sailing or canoeing or something."

\section{Code: 8 Measure indiv}

Brief Definition: Measures more individual

Definition \& representation of the scenario (opinion of Mr. Müller): I would like to continually expand and improve the health promotion of my employees. Are there any other ideas? Question within focus-group guideline: Q11. Mr. Müller would like to continually expand and improve the health promotion of his employees. Are there any other ideas?

RQ $1 \& 2$ \& 3: Contributes to define salutogenic factors that can be generated by top management to support the promotion of health and well-being of workforce, contributes to develop top management awareness and also contributes to understand how SMEs in EWL/Germany integrate health promoting and well-being factors.

Example: „[...] it must be easy to fit into the company, so it must be an individual solution that is tailored to the company and the teams in the company, that everyone can participate in it, and that there may be measures that everyone can make, where everyone has something from it [...].“ 


\subsection{Partial of focus-group transcripts}

The two focus groups and the reflection group were transcribed and partial listed below.

\subsubsection{Focus-group discussion top managers}

Partial transcript which shows some of the codes:

\section{Legende der Sprechenden:}

W1: Frau Julia Pönighaus, Moderatorin

W2: Frau K., Assistentin

W3: Frau H.

M1: Herr D.

M2: Herr S.

M3: Herr W.

M4: Herr R.

M4:

[...] also wir müssen doch ein Vorbild sein, natürlich haben wir ein Privatleben, aber das darf nicht ins Geschäftliche - finde ich persönlich, vielleicht bin ich auch altmodisch, aber das darf man nicht ins Geschäftliche rein tragen, dass ich meine jetzt muss ich meine Frau besuchen und die Kinder.

M1: $\quad[. .$.$] natürlich kann jeder seine Mittagspause nutzen wie er will.$ Der eine raucht, der andere schläft. Und wann man dafür Betten, einen Schlafsaal zur Verfügung stellen müsste, weil da die Nachfrage ist, ich glaube da hätten wir alle nichts dagegen.

M2:

Ja, also 100\%ig, aber nur eine kleine Einschränkung: Sie sagen „Von oben“: Unternehmenskultur wird immer ganz stark geprägt durch - wir sind hier ja Mittelstand - durch den Unternehmer, durch Sie persönlich, durch Sie als Unternehmerfamilie, aber Unternehmenskultur, sage ich allen Mitarbeitern und Mitarbeiterinnen, das sind wir alle, d.h. also jeder ist Baustein, aber Prägung, so diese DNA, wird natürlich 
auch durch den Unternehmer, die Unternehmerfamilie gelebt, das muss ich schon sehen.

M2:

Selbstverantwortung, da sind zwei Sachen in dem Begriff drinnen, das fängt mit selbst erst mal an.

M3:

Ein Punkt, der uns aufgefallen ist, das hat aber mit der Größe des Unternehmens zu tun. Was sicherlich immer schwierig ist, ist Mitarbeitern von oben herunter, das haben wir schon gehabt, so "Chef schlägt vor" oder "Chef schreibt gar vor: Mach mal das und das", oder "Lasst uns mal das und das machen." Was wir festgestellt haben, was bei uns - das geht so ein bisschen Richtung feel good, aber eben dann auch Richtung Bewegung und das Überbringen von verschiedenen Möglichkeiten gibt es auch noch, was zu tun ist: Gemeinsame Veranstaltungen zu machen. Ich bin eigentlich kein Freund davon, Arbeit und Privates zu vermischen. Was wir aber festgestellt haben ist, dass wir bessere Stimmung im Unternehmen haben, wenn wir regelmäßig gemeinsam was tun. Das lässt sich in großen Unternehmen nur teilweise machen, weiß ich. Ich habe es früher bei der Firma XX mit meinen 20 Leuten gemacht, und mache es heute mit der eigenen Firma mit 20 Leuten. Das tun wir zu wenig. Wenn wir es aber machen, stellt sich raus, dass die Leute das sehr positiv wahrnehmen, Richtung feel good, und gleichzeitig man ins Diskutieren kommt, "Mensch, das könnte ich privat auch mal machen." Und dann sind wir bei diesem Thema Bewegung, weil wenn wir was machen, dann machen wir Bewegungssachen, wir machen Fahrrad-, Sagway- oder Kanu fahren oder so was. Wir machen das viel zu selten. Die Ideen sind alle da, aber das ist eine gemeinsame Unternehmung, die Richtung Gesundheitsdenken geht oder zumindest eine Empfindung stärkt, die es bei vielen dieser Punkte würde es von oben herab, glaube ich, noch gar nicht so gibt, also von oben herab? Die Leute erreiche ich besser damit, glaube ich. 


\subsubsection{Focus-group discussion employees}

Partial transcript which shows some of the codes:

Legende der Sprechenden:

W1: Frau Julia Pönighaus, Moderatorin

W2: Frau K., Assitentin

W3: Frau O.

W4: Frau W.

M1: Herr S.

M2: Herr St.

M3: Herr H.

M4: Herr S.

W3:

Als ich damals bei der Firma $X$ angefangen hatte, habe ich darauf gedrängt, den Betrieb auch kennenzulernen, und dann wurde mir auch die Möglichkeit gegeben, dass ich eine Woche im Betrieb sein durfte, weil mir das ganz wichtig war, alle Abläufe kennenzulernen, ja, was mir dann natürlich hinterher in meiner täglichen Arbeit sehr viel weiter hilft.

W4:

Also man braucht ja kein Sternchen im Klassenbuch oder was da jetzt gerade steht, aber einfach ein ganz normales Danke, das finde ich einfach schon das Netteste. Und es ist ja auch blöd, wenn du dich durch Lob veräppelt fühlt, so, „Wie meint er das jetzt, hat der das vielleicht ironisch gemeint?", wirklich, es heißt ja auch immer, jeder ist ersetzbar und das wird auch, kann auch knallhart durchgezogen werden. Es suchen ja genug Leute Arbeit, und wenn einer nicht wirklich mitspielen möchte, dann wird er ausgetauscht. Ist halt so.

M4:

Also die Punkte waren, die Ideen und die Punkte, die der Herr Müller umgesetzt hat, waren alle gut, es muss einfach passen zum Unternehmen, also es muss eine individuelle Lösung sein, die auf das Unternehmen und auf die Teams auch im Unternehmen irgendwie zugeschnitten ist, dass auch jeder daran teilnehmen kann, und dass es vielleicht Maßnahmen 
gibt, die jeder machen kann, wo auch jeder was von hat, dass erst mal gemeinsam alle was davon haben, um dann vielleicht auch noch mal zu gucken, welche Abteilung, welche Arbeitsgruppen kann ich dann wieder bilden, die dann gezielte Förderung für irgendwas kriegen. Man muss gucken, wo habe ich eher körperliche Belastung, zum Beispiel in der Spedition, im Lager, im Versand; und die Leute aus dem Büro dann Rückentraining und so, solche Geschichten, dass es einfach passt und so gestaltet ist, dass man es nutzen kann, Spaß hat, das irgendwie zu nutzen. Ja, wenn der Vorgesetzte, also am besten der direkte Vorgesetzte das vorlebt, umso einfacher, ja. Es ist sehr schwierig, einen speziellen Punkt rauszufiltern. Also der Vorgesetzte als Vorreiter, klar, so eine Funktion jetzt nicht. Wenn ich da so ein Konzept habe und mein Vorgesetzter hält da gar nichts von und sagt, „Oh, jetzt machst du wieder eine halbe Stunde während der Arbeitszeit dein Training", dann funktioniert das am Ende nicht. Vielleicht sogar einer mit der wichtigsten Punkte. 


\subsubsection{Reflection focus-group discussion}

Partial transcript which shows some of the statements linked to the findings:

Legende der Sprechenden:

$\mathrm{F} 1=\mathrm{Mo}$

$\mathrm{F} 2=\mathrm{Me}$

$\mathrm{F} 3=\mathrm{Ma}$

F3:

Also Führen durch Vorbild finde ich auf jeden Fall, das ist immer noch aktuell; in kleineren Betrieben wird das der Chef sein oder in größeren dann eben diese untergeordnete Führungsebene, aber das ist für etwas, was überall aktuell ist; und die Mitarbeiter werden sehr wohl wahrnehmen, ob der Chef mit dem Auto kommt oder mit öffentlichen Verkehrsmitteln oder mit dem Fahrrad. Also ich finde nonverbal ist das ein gutes Zeichen.

F2:

Oder anders herum, wenn der Chef einen guten Kontakt zu dir hat, ins Gespräch geht, und dich natürlich auch kennenlernt mit deinen Stärken und Schwächen.

F3:

Also ich glaube auch, das ist das $\mathrm{A}$ und $\mathrm{O}$, dass man die Menschen kennt und nur so geht das, dass man die Schwächen und die Fehler oder die guten Seiten unterstützen kann.

F1:

Ja, in jedem Fall, in jedem Fall. Und ich glaube das ist auch den Mitarbeitern ja ganz wichtig, dass, der Austausch mit der Chefetage ist denen so wichtig, weil sie wissen, nur der kann etwas ändern. Also ich denke auch für die meisten hier, man braucht halt einen direkten Kontakt zum Chef, um was zu ändern. Ich glaube da schieße ich mich an, das ist das $A$ und $O$. Und wenn es ein Chef ist, der natürlich nachvollziehen kann, dass ich jetzt gerade nicht das mache, dass mache ich vielleicht ja, in Ordnung, aber andere Sachen kann ich viel besser und effizienter erledigen. Also das geht glaube ich nur durch den persönlichen Kontakt.

F1: Also was mich etwas überrascht hat oder sehr überrascht hat, dass einer sagt, „Einen ganz tollen Auszug habe ich gesehen, 
,Ich habe den Trump gewählt, weil ich gerne wieder möchte, dass alles wieder so wird, wie es früher mal war'“. Und das ist eigentlich die Antwort: Die Leute sehen sich nach Früher. Also da kann man sehen, welche Unsicherheit auch da ist und welche Ängste da sind, die man vielleicht gar nicht befriedigen kann. Also das war für mich schon eine Aussage, die mich sehr gewundert hat.

F2:

Antiquiertes Führungsverständnis, also mehr so der „früher war noch vom Patriarchen die Rede", "Ich bin der Unternehmer, ich führe das, und hier läuft's wie ich mir das vorstelle, ich gebe regeln vor".

F3:

Und so jemand verkörpert eine Sicherheit - ob die trügerisch ist, ist eine andere Sache. Aber sicher zu sein, dass man da, wo man mal angefangen hat zu arbeiten, bis 65 bleibt, das erträumen sich viele, aber das wird es nicht mehr geben.

F3:

Ich glaube das ist den Führungskräften total aus dem Blickfeld gekommen, dass Mitarbeiter Zuwendung, Wertschätzung brauchen, weil der finanzielle Druck so groß ist und der Zeitdruck, der hat sich so enorm gesteigert durch den Computer, wovon ich ja mal gedacht habe, da hätte man mehr Zeit, nein, im Gegenteil. Die Menschen sind ja so gehetzt und ich glaube, das war vor 40 Jahren noch anders. Da kannte der Chef seine Mitarbeiter, die Firmen waren nicht so riesig groß und global, sondern da hat man in der Holzfabrik, derjenige, der da das erste Stück Holz gesägt hat, der hat nachher auch den Stuhl gesehen, der da produziert wurde. Also ich glaube so eine ein bisschen überschaubarere Bereiche, die haben das früher einfach einfacher gemacht, dass der Chef seine Mitarbeiter kannte, und der wusste auch, wenn die Frau gestorben war oder ein Kind krank war oder so und konnte darauf eingehen, das war früher eben einfacher zu bewerkstelligen und ist heute schwieriger in diesen großen Firmen, und dieses Bewusstsein, das müsste wieder mehr geweckt werden. 


\subsection{Slides of the focus-group discussions}
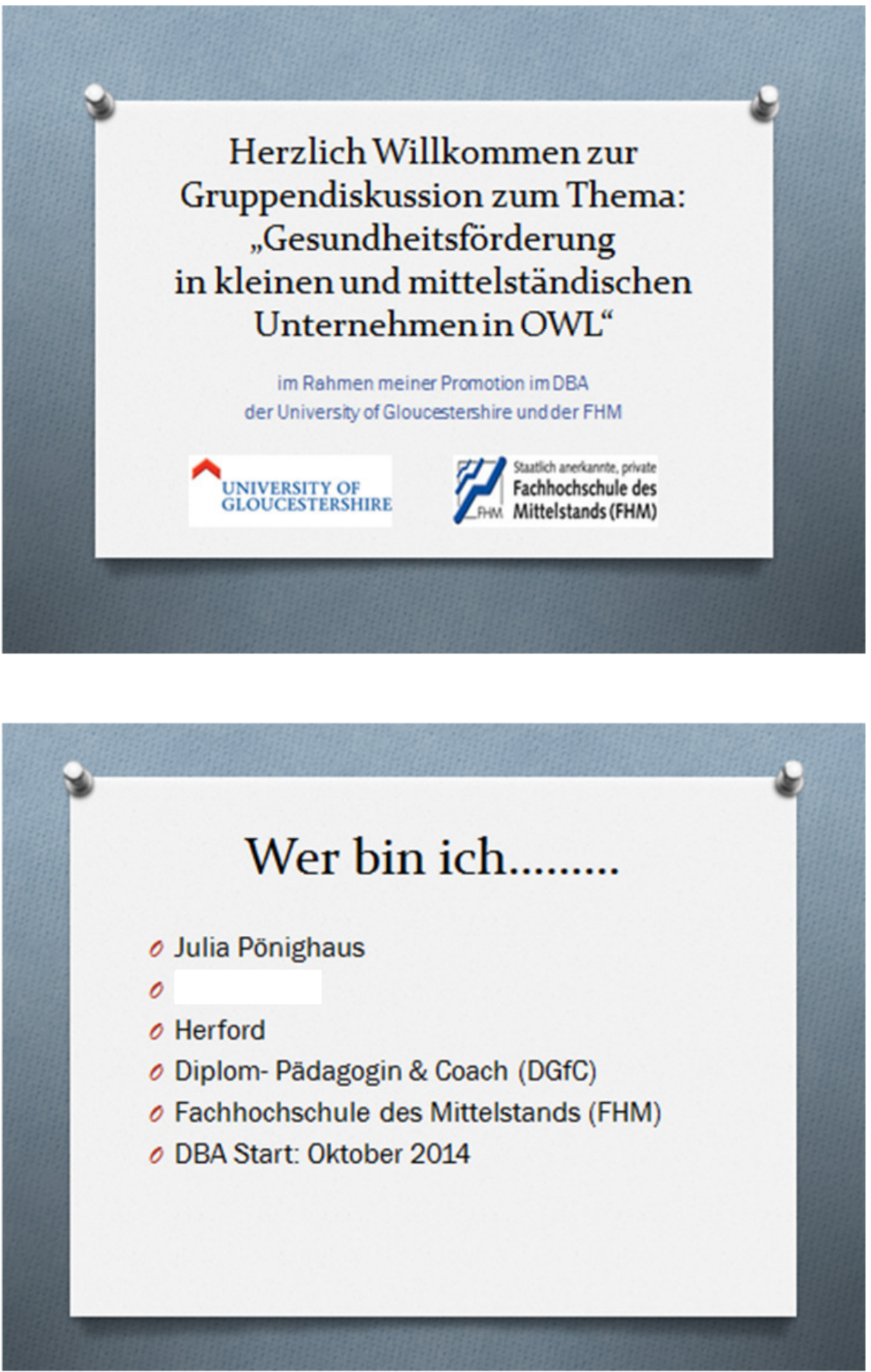

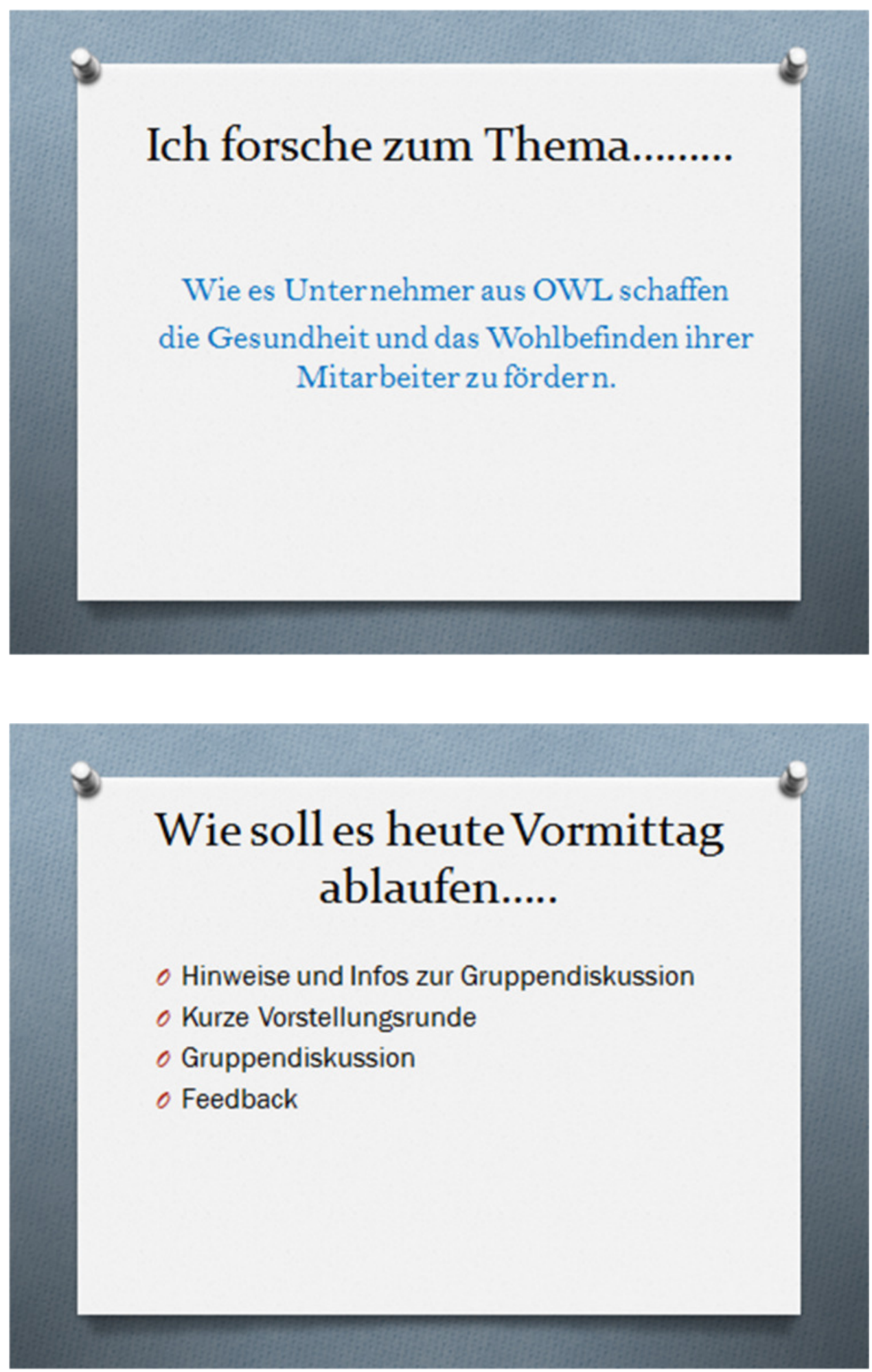


\section{Lassen Sie uns folgendes vorstellen......}

o Wir befinden uns im Jahr 2046

0 Vieles hat sich verändert, auch in der Wirtschaft

o Auswirkungen der Demografie, des Fachkräftemangels, der Globalisierung und der Standortqualităt sowie die Digitalisierung und der technologische Wandel zeigen sich deutlich

o Daher hat ein Umdenken in allen Bereichen stattgefunden

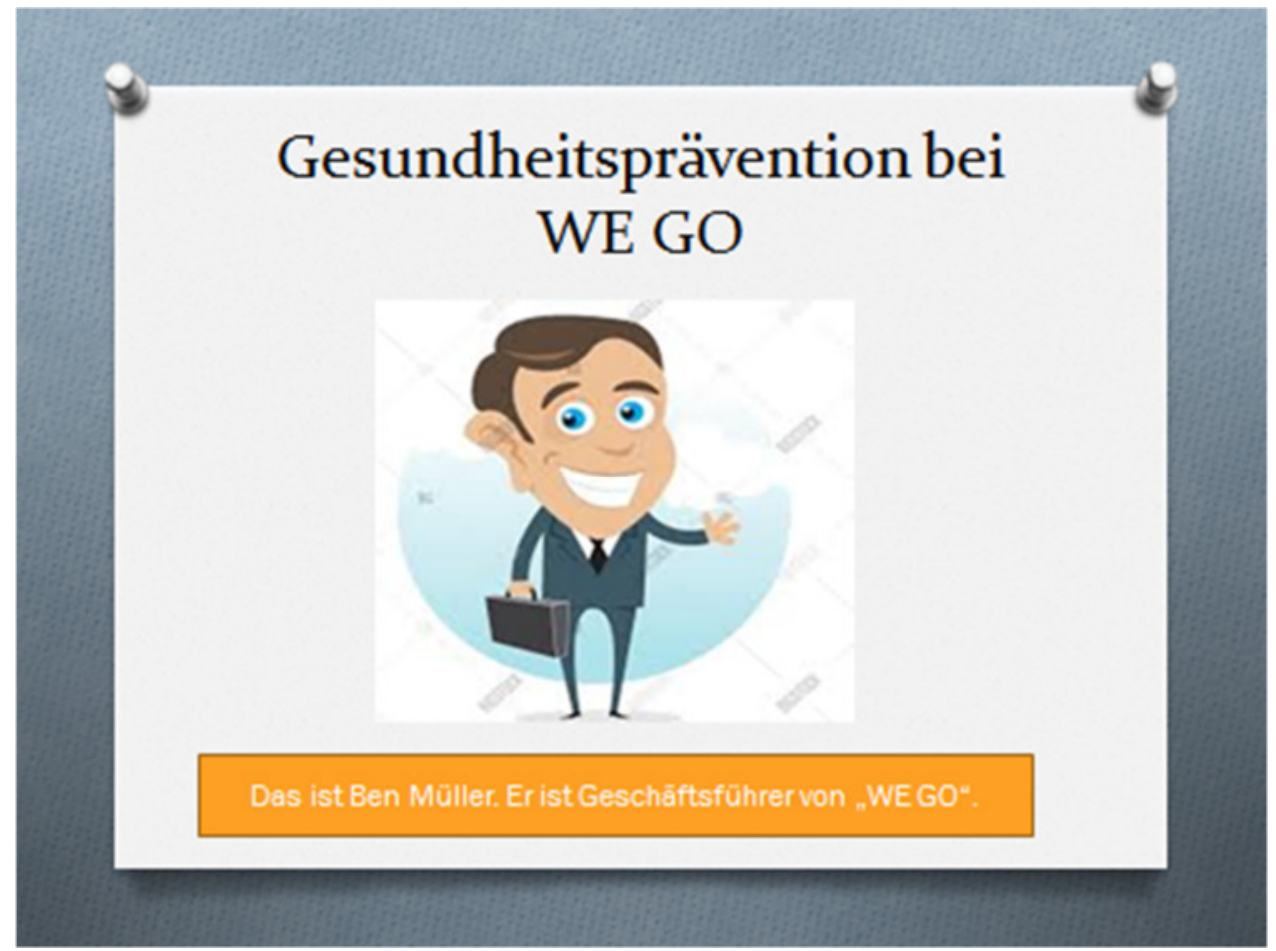



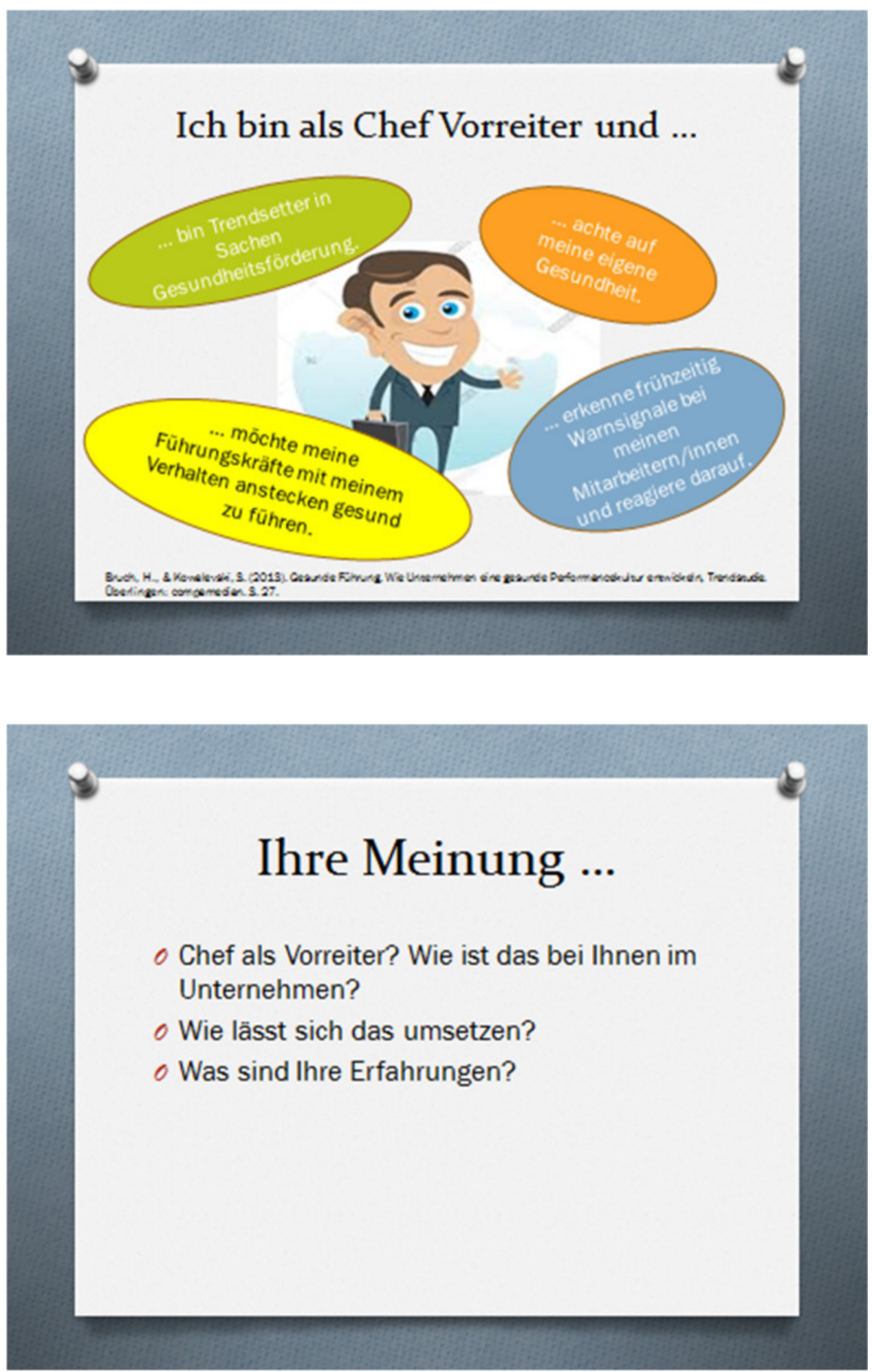

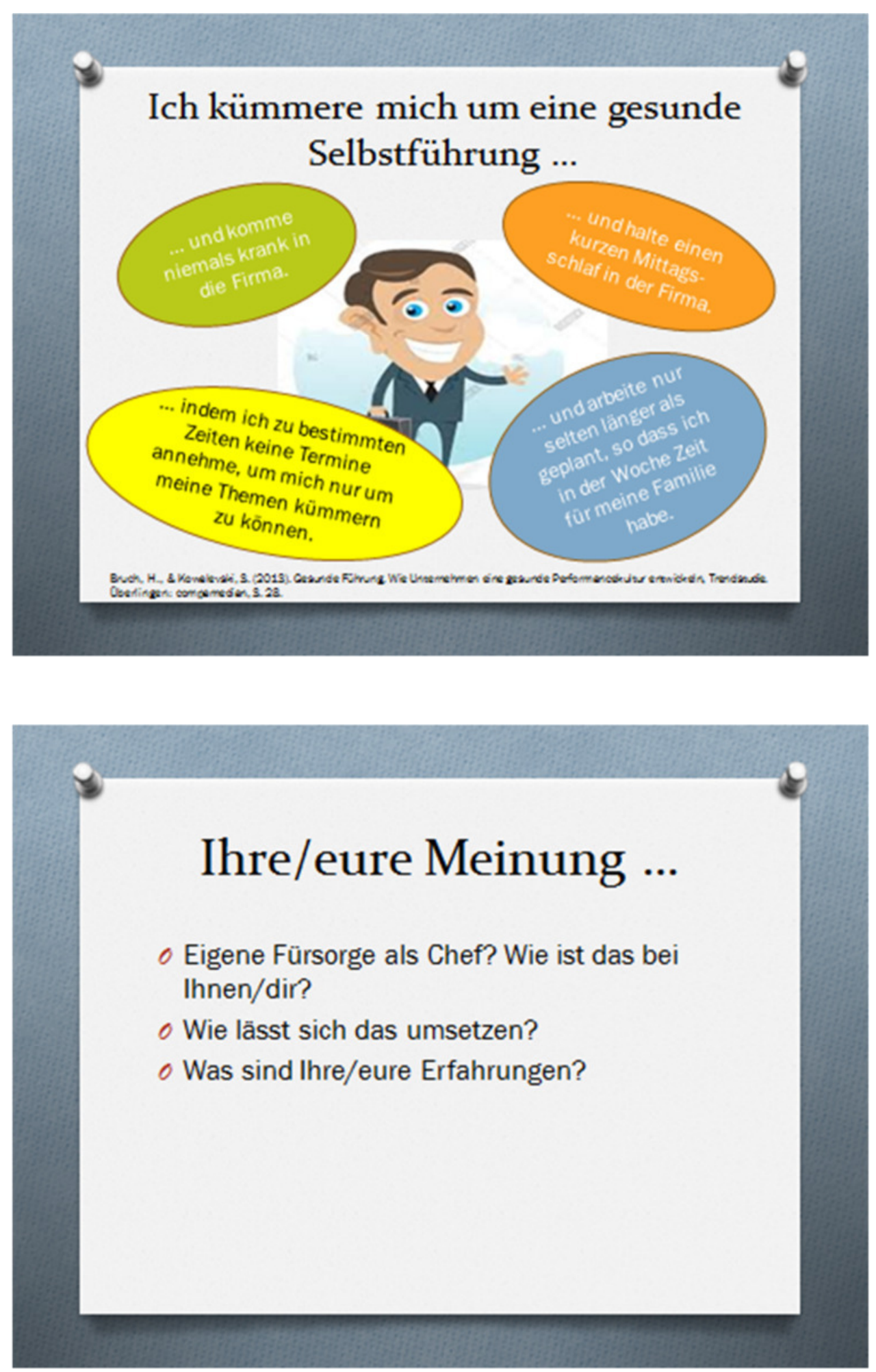

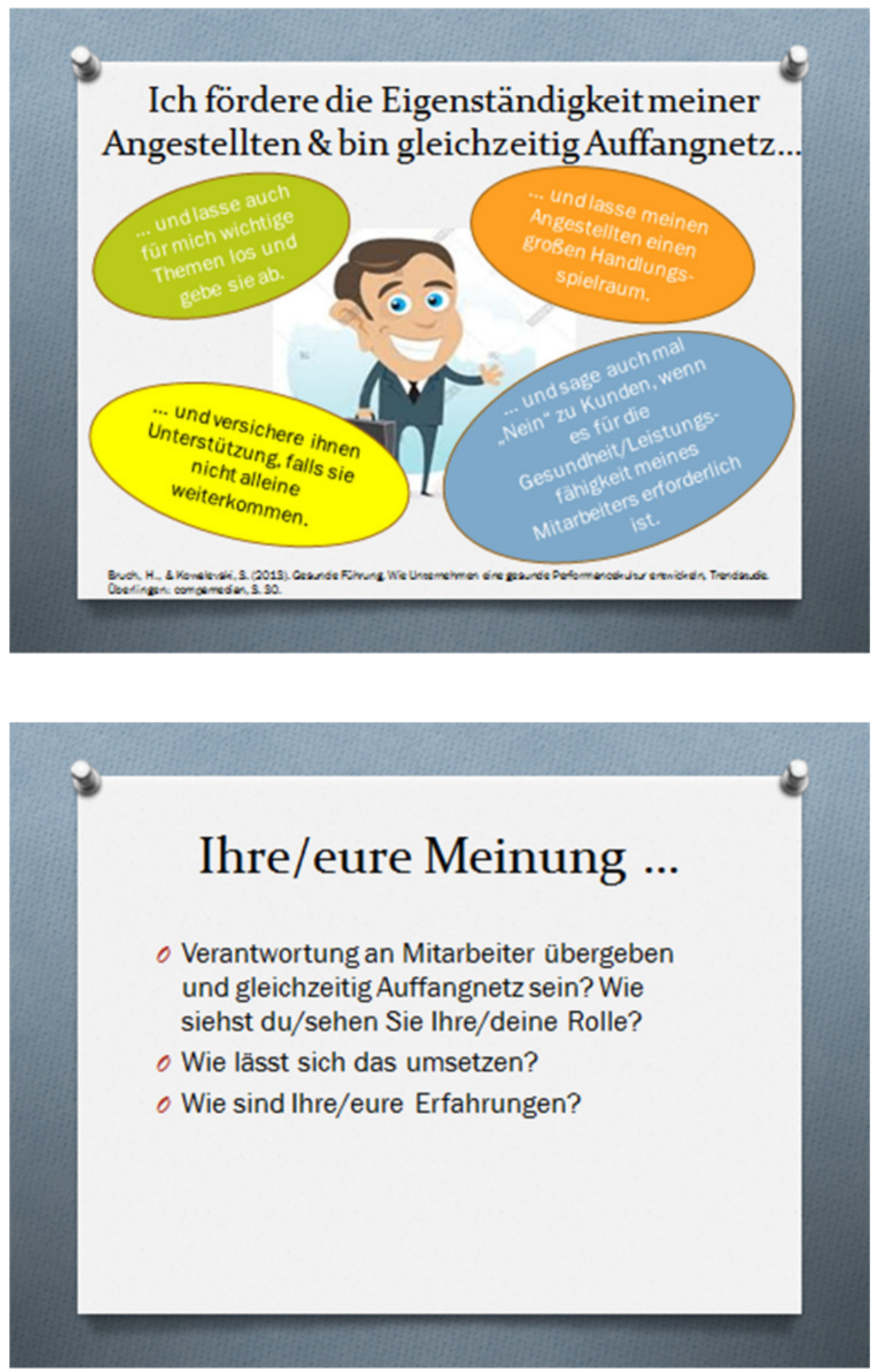

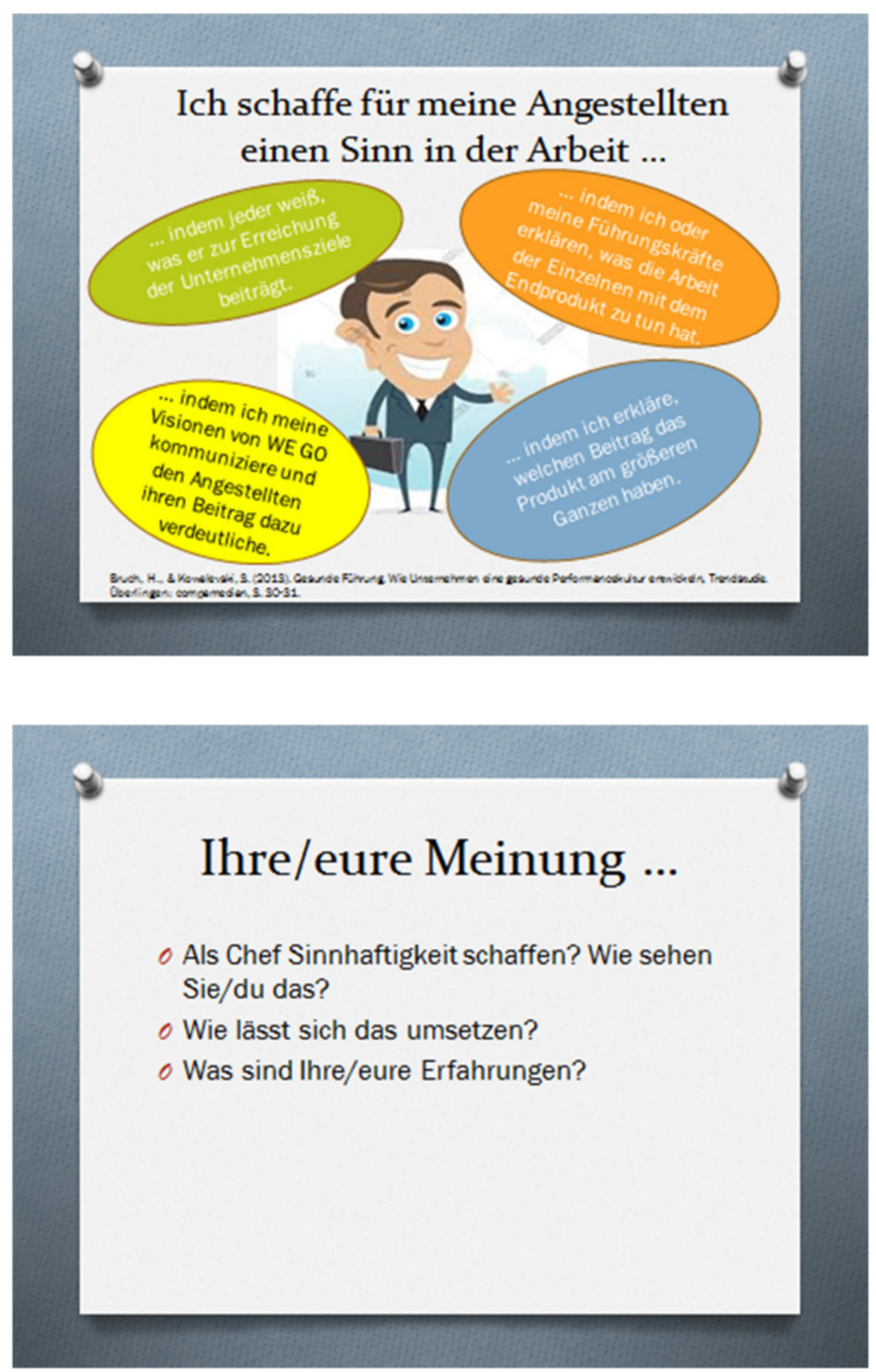

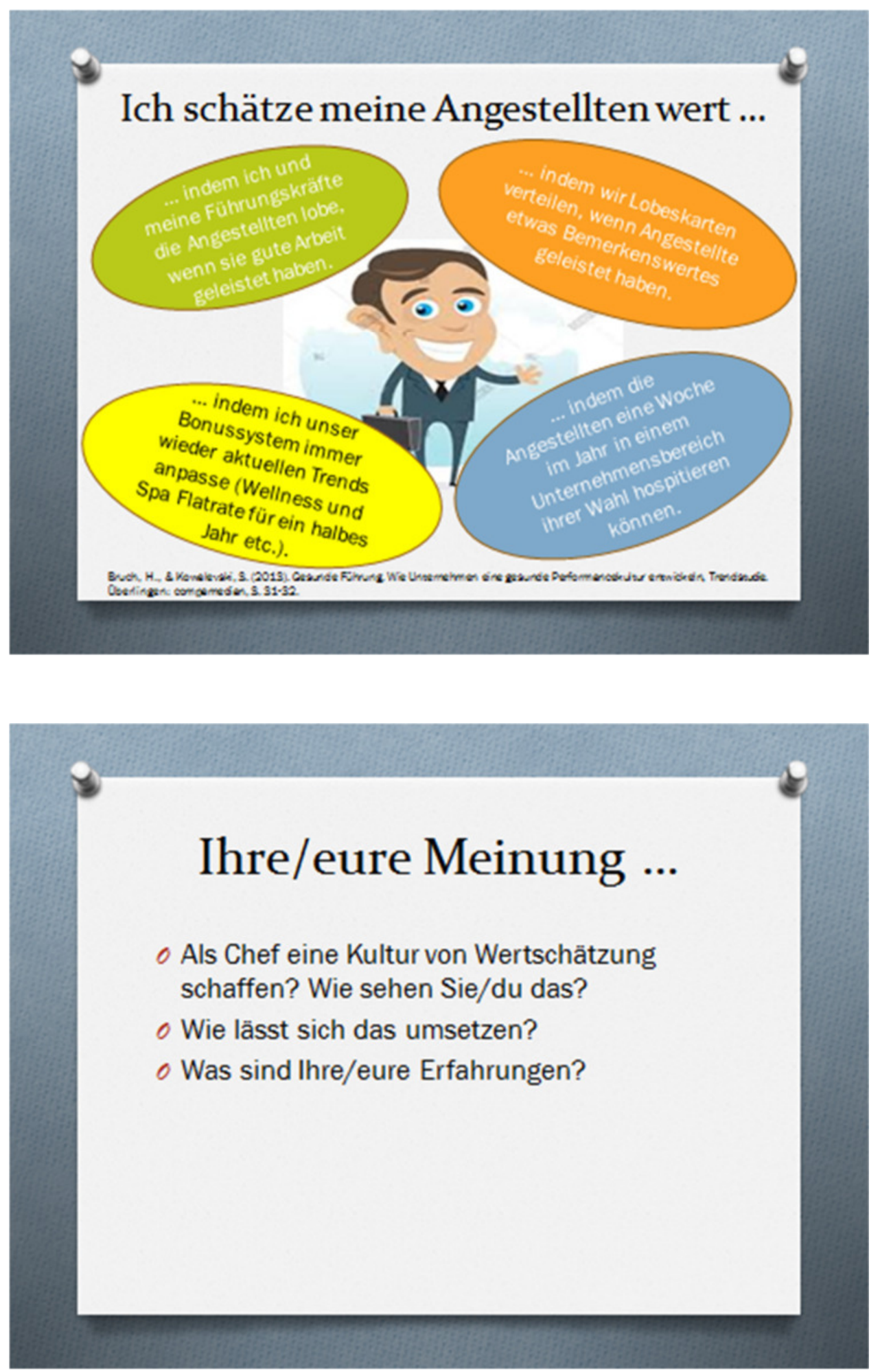


\section{Ich fordere und fördere meine Angestellten .}

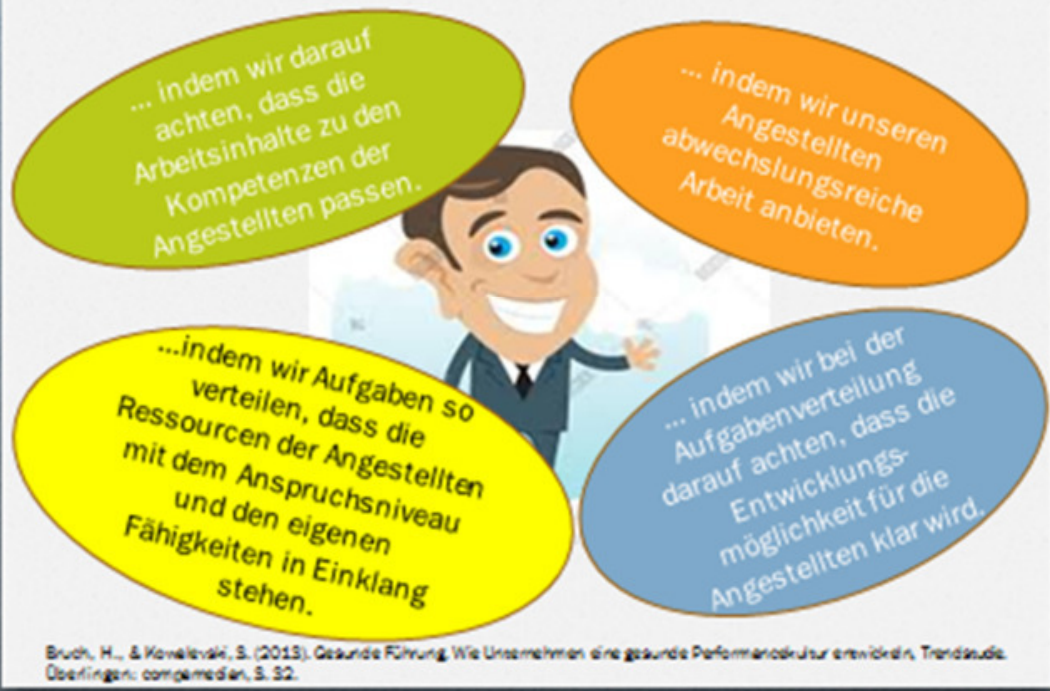

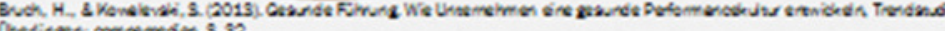

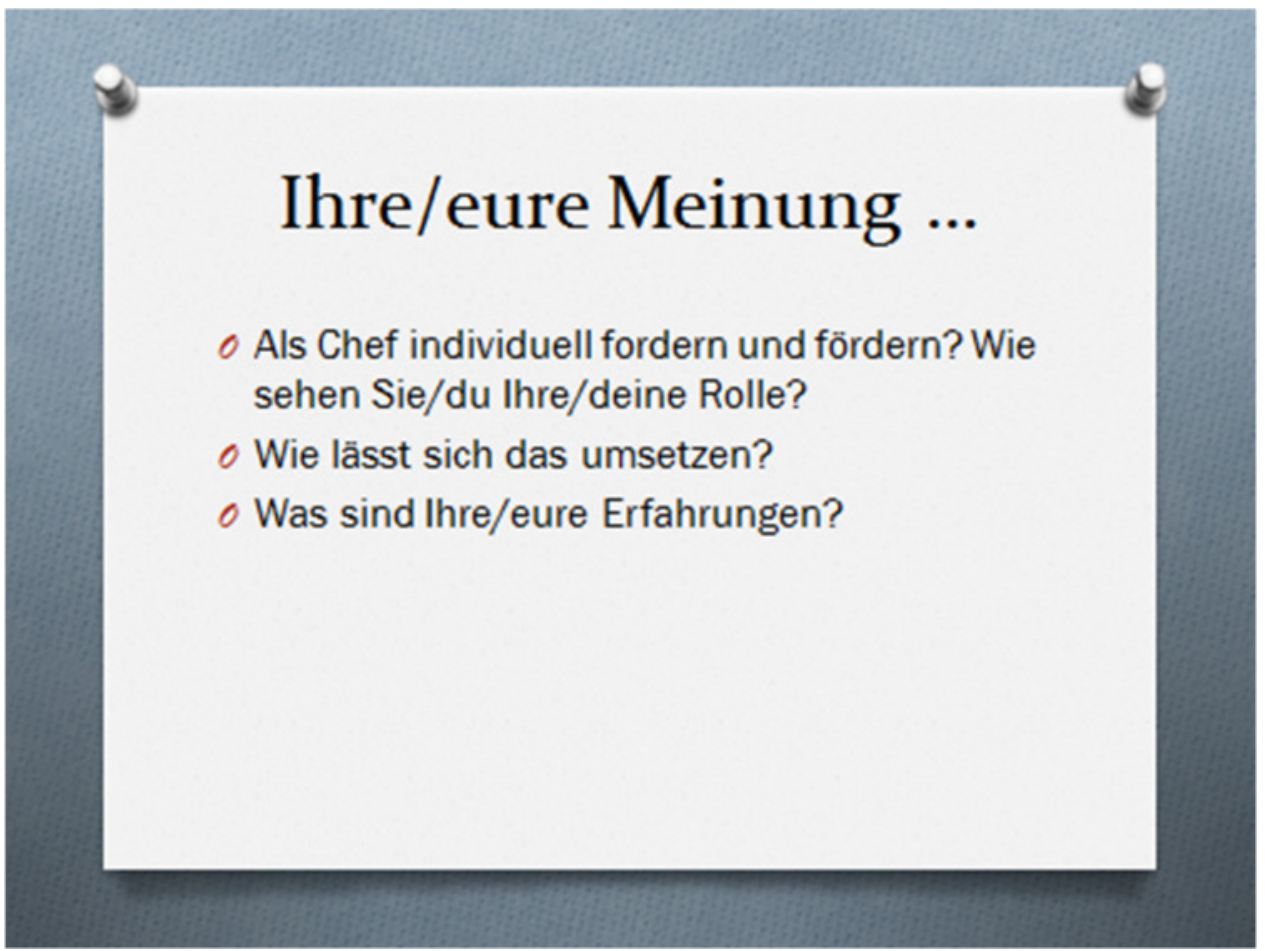



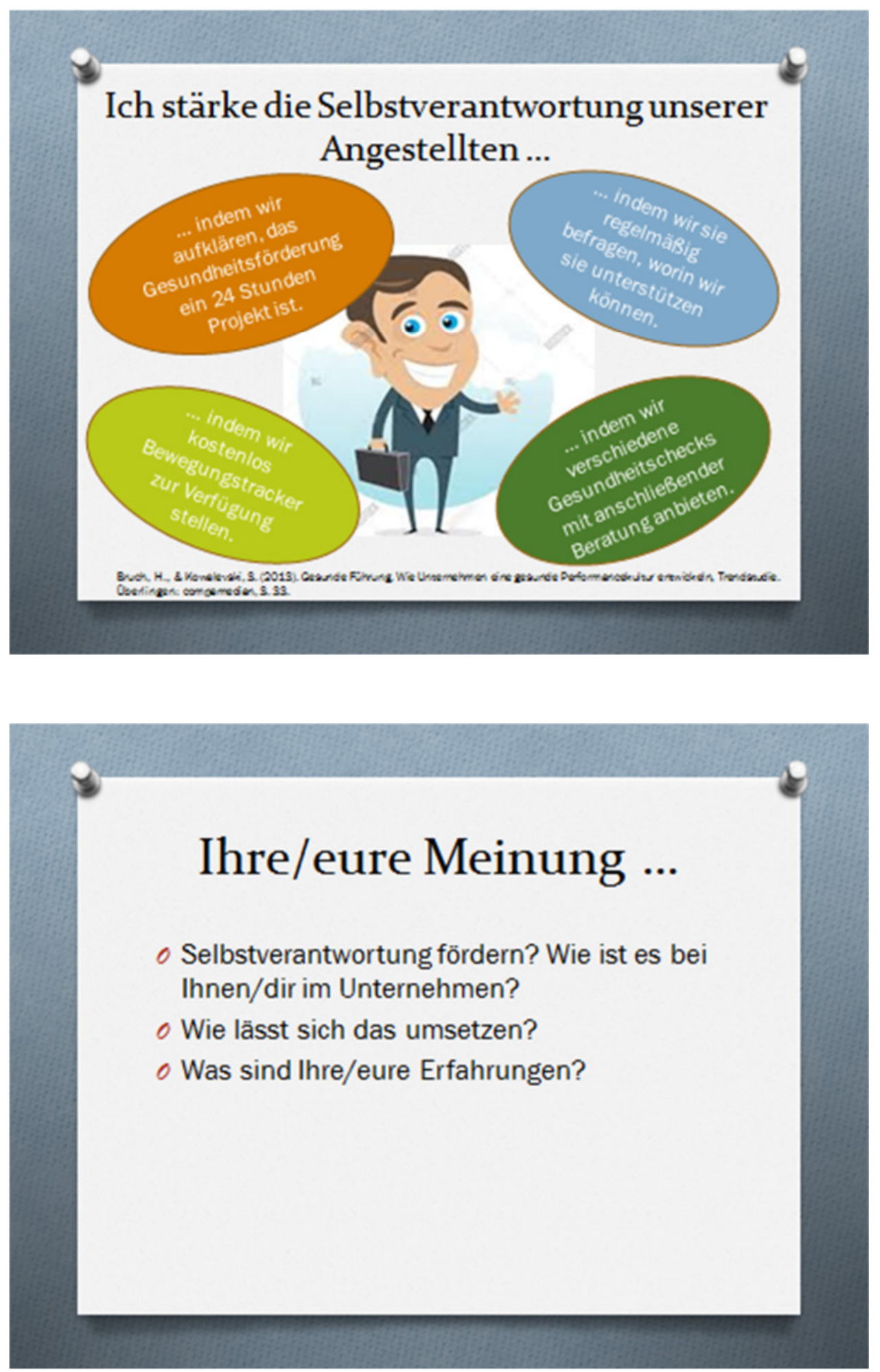

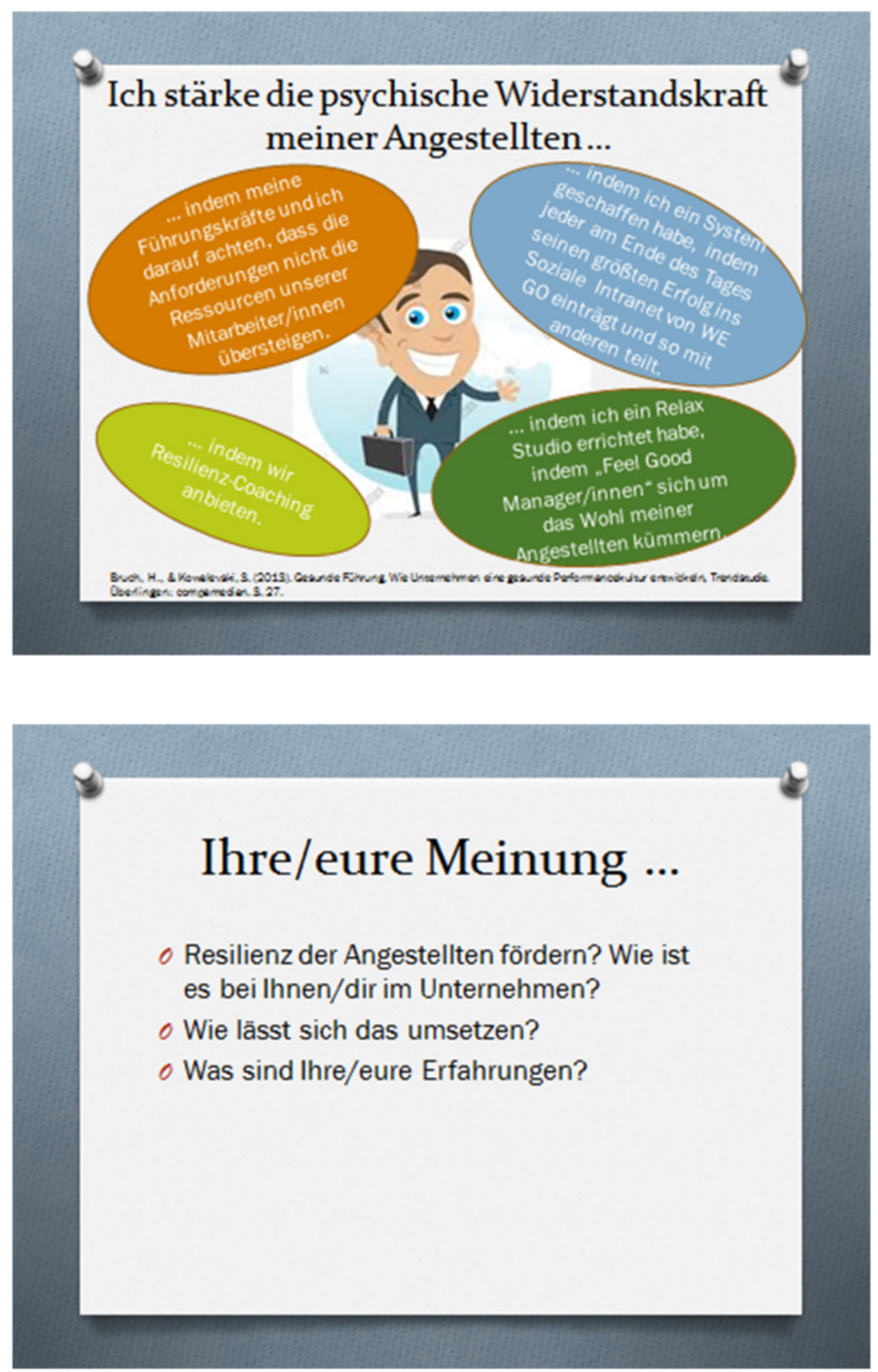

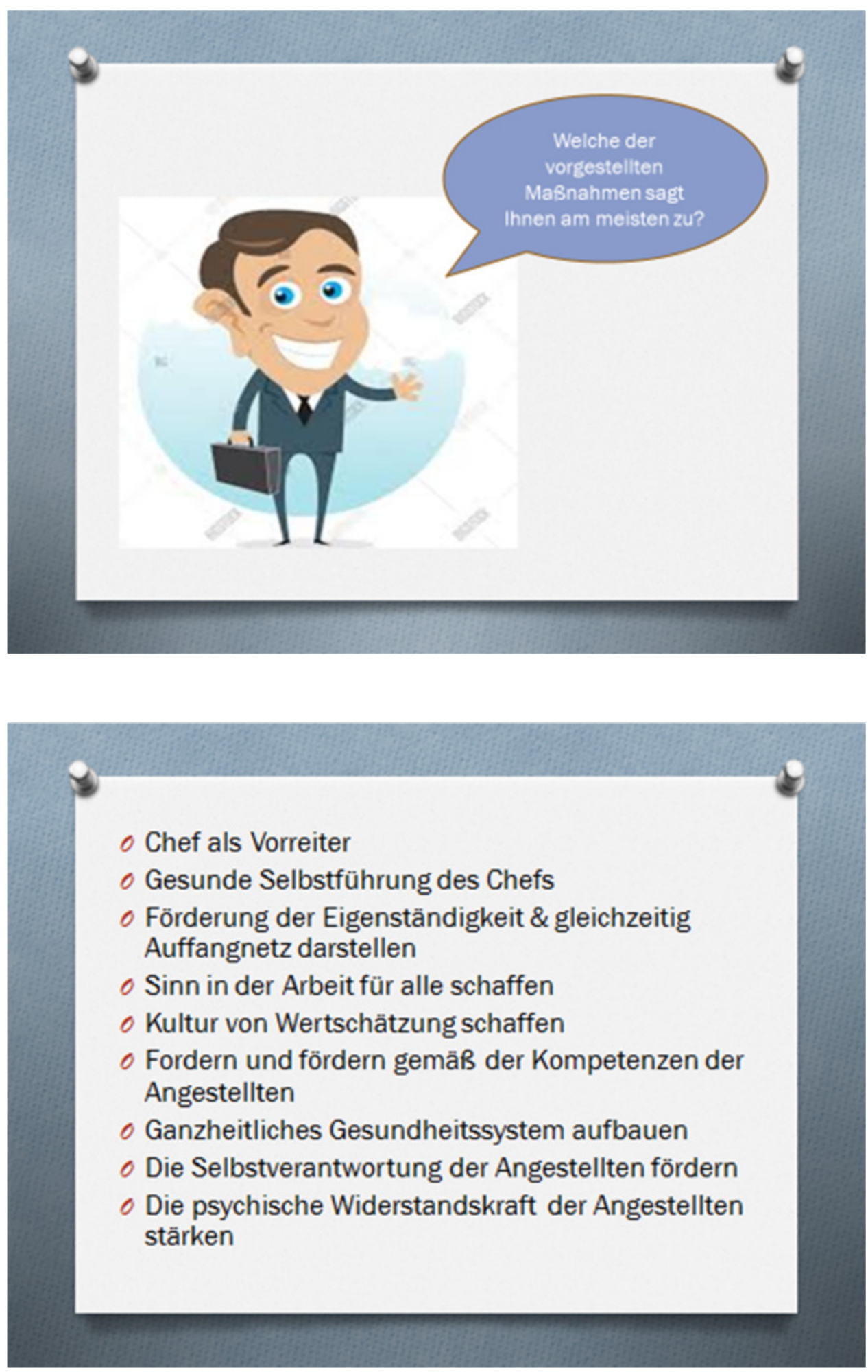

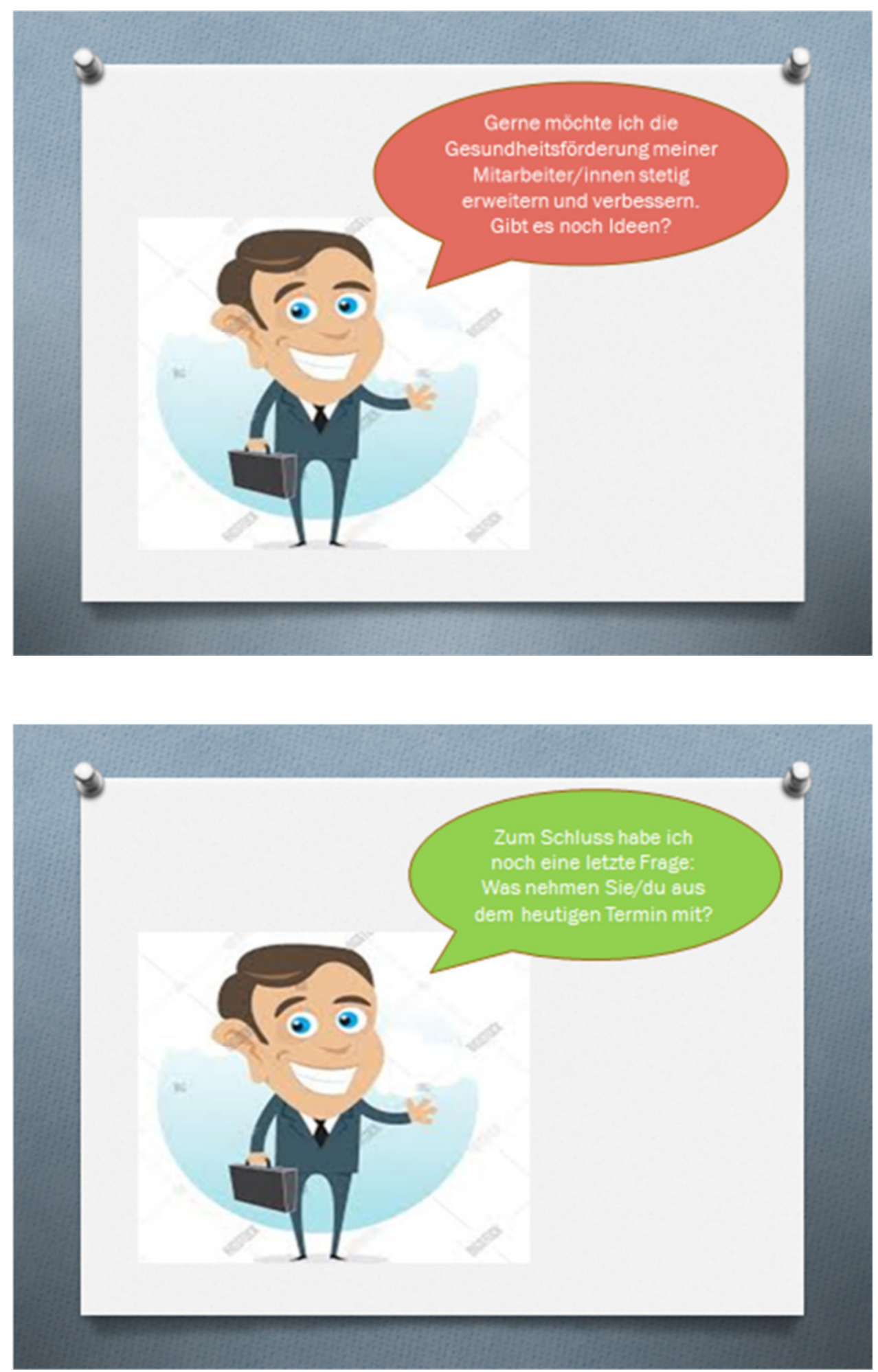


\section{Herzlichen Dank für Ihre/eure Unterstützung und das Vertrauen!}




\subsection{Summary of focus-group discussion data for the reflection group}

\section{Slide}

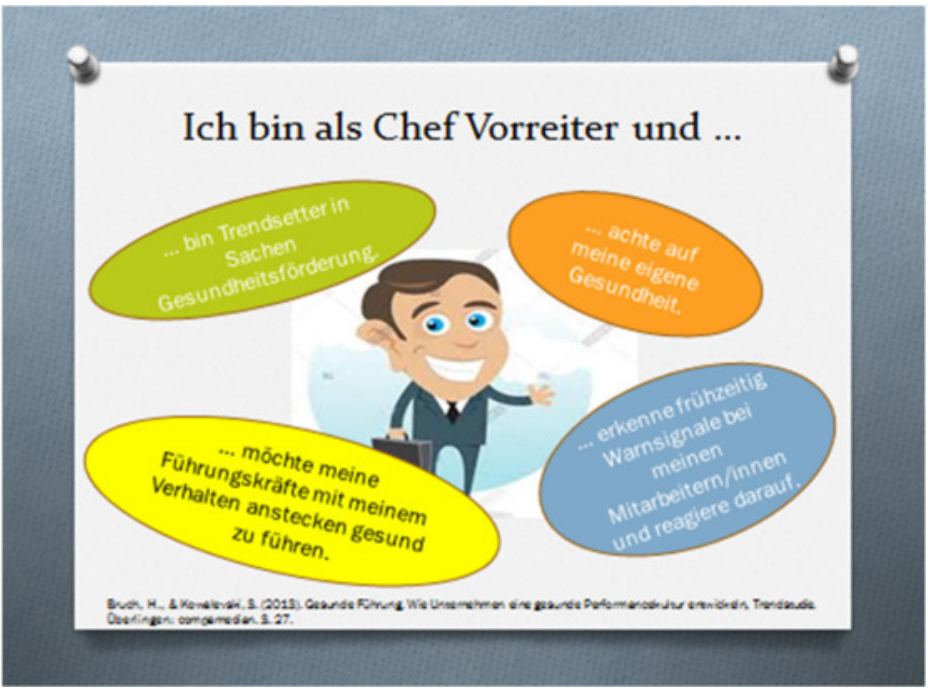

\section{Top Management:}

.....Man muss Vorreiter sein, indem man dann irgendwie - Zwang ist jetzt falsch, aber was macht, wo sie mitmachen müssen, wenn man denn meint, dass das gut für sie ist. Freiwillig kommt da nichts

....es gibt eine Grenze, und die Grenze heißt, jeder ist am Ende des Tages auch erst mal selbst verantwortlich für sich, sich gesund zu erhalten,.....

...Ich finde es gut, wenn der Geschäftsführer oder der Vorgesetzte, egal welche Strecke, auf dem Fahrrad kommt, während der Mitarbeiter im tiefer gelegten 86er Audi seine anderthalb Kilometer mit dem Auto kommt.

... wir sitzen quasi uns gegenseitig auf dem Schoß in so einem kleinen Unternehmen, da ist die Vorreiterrolle des Chefs, die würde extrem wahrgenommen werden, wenn ich mit dem Fahrrad käme, das würden alle jeden Tag sehen und auch jeden Tag wahrnehmen. Wenn ich jeden Nachmittag um vier sagen würde, ich muss jetzt - nicht jeden, aber einmal die Woche, jede Woche um vier sagen würde, ich muss gehen, ich habe Rückenschule, ich habe Sport oder ich gehe morgens schwimmen vor der Arbeit, wie ich das als Student immer jeden Morgen gemacht habe, das würde definitiv wahrgenommen werden und hätte dann eine Vorbildrolle, überhaupt keine Frage. Der Chef tut was, das wird den Mitarbeitern, unterstelle ich jetzt mal, bewusst sein, „der Chef tut was, ich für mich nicht". Unterschied ist definitiv, wir haben keine Anspruchshaltung.

... Also bei wenigen Mitarbeitern ist die Nähe viel größer, aber ich glaube, was uns gemeinsam ist, ist das Thema Vorbildfunktion, das ist in beiden Bereichen, ob groß oder klein - die Nähe macht die Wahrnehmung glaube ich noch mal anders. 
... aber quasi jetzt so ein Thema wie "Work-Life-Balance“ so aus dem Gleichgewicht zu nehmen, dass ich sage ich bin jetzt total in der Balance und bin total entspannt, das ist denke ich der falsche Weg und ist auch ein falsches Vorbildfunktion.

... eine Frage war "Führen durch Vorbild“, das ist ja eigentlich so ein bisschen abgeleitet dadurch, dass wir sagen, ich habe so eine Kaskade, nicht nur der Inhaber führt durch Vorbild, sondern er braucht ja auch Multiplikatoren. Das steckt ja hinter diesem Thema Führungskräfteentwicklung, wie das quasi bis in alle Ecken des Unternehmens getragen wird.

... also der Chef ganz oben müsste für mich jetzt nicht die Vorreiterrolle haben, sondern das müsste auf die Führungskräfte runtergebrochen werden.

\section{Mitarbeiter:}

... Also eine gewisse Vorbildfunktion zu übernehmen beim Thema Gesundheit zu übernehmen ist mit Sicherheit von Vorteil meiner Ansicht nach, nur die Krux liegt darin, die Mitarbeiter mitzureißen bei diesem Thema

... Ich glaube so im Bezug auf sich selber nimmt man das gar nicht so wahr, also bei uns ist es zum Beispiel so, unsere beiden Chefs kommen die größte Zeit mit dem Fahrrad zur Arbeit, aber es sind halt die Chefs, die kommen mit dem Fahrrad zur Arbeit, aber das auf sich selber zu übertragen, das findet glaube ich nicht so automatisch statt.

... Ich glaube auch nicht, dass viele Mitarbeiter das wahrnehmen würden, was der Chef macht, weil sie davon viel zu weit weg sind - ob der mit dem Fahrrad kommt, oder ob der mittags Salat ist. Weil wahrscheinlich die räumliche Abgrenzung zu groß ist.

... Also bei uns ist es auch so, dass der Chef eigentlich rund um die Uhr einsatzbereit, einsatzfähig ist, und ich finde, dass sich das schon mehr auf die Mitarbeite überträgt als das Thema „ich gehe die Treppe" oder "ich komme mit dem Fahrrad zur Arbeit", weil das auch ein bisschen Druck natürlich auch ausübt, dass man selber auch sagt, „Mensch, der Chef, der kommt krank zur Arbeit, der macht dies, der macht das, und man selber gibt sich dann doch Mühe, da auch irgendwo ein bisschen mithalten zu können manchmal, denke ich, was auch nicht ganz positiv dann vielleicht ist....

Wenn ich da so ein Konzept habe und mein Vorgesetzter hält da gar nichts von und sagt, "Oh, jetzt machst du wieder eine halbe Stunde während der Arbeitszeit dein Training“, dann funktioniert das am Ende nicht.

... Ich denke nur der Chef alleine wird nicht ausreichen. Da muss man glaube ich ein größeres Spektrum finden, Abteilungsleiter erst mal mitziehen, also nur dass der Chef was vorgibt glaube ich wird nicht ausreichen, wenn man in einem Unternehmen von der Größenordnung ist, wie wir es haben.

... Aber als Chef hat man natürlich auch die Möglichkeit, Kleinigkeiten zu bewerkstelligen wie Obstkörbe vielleicht aufstellen und Sachen zu fördern oder Unterstützung zu geben

... Man müsste den Mitarbeitern irgendwie eine Anregung geben oder so, dass sie sich einfach mal damit beschäftigen, auch während der Arbeitszeit. 
... Auf der anderen Seite sollte eine Bereitschaft oder auch eine, ja, vom Chef auch irgendetwas kommen, also dass der Druck nicht größer wird wie zum Beispiel psychische Schäden, die man - was ja auch immer schlimmer wird, wie eben schon gesagt, Burn-out oder sowas, dass man da auf die Mitarbeiter achtet, dass die nicht sich selber so unter Druck gesetzt fühlen und dann lieber krank zur Arbeit kommen, weil sie Angst haben, gekündigt zu werden, dass in der Hinsicht der Mensch ein bisschen mehr zählt und - aber ob ich jetzt ins Fitness-Studio gehe oder nicht, das muss jeder ja für sich selber entscheiden.

... Also ich finde auch, dass man ja auch das trennt: privat und Arbeit. Und vielleicht die Arbeitssachen gar nicht so viel Einfluss auf das private Leben haben. Bei uns weiß auch jeder, der Chef geht fünf Etagen jeden Morgen die Treppe hoch. Trotzdem fahren alle anderen mit dem Fahrstuhl. Wenn es vielleicht im privaten Umfeld was gibt, ich glaube da wird man leichter beeinflusst für sich selber, persönlich, als bei der Arbeit.

\section{Slide}

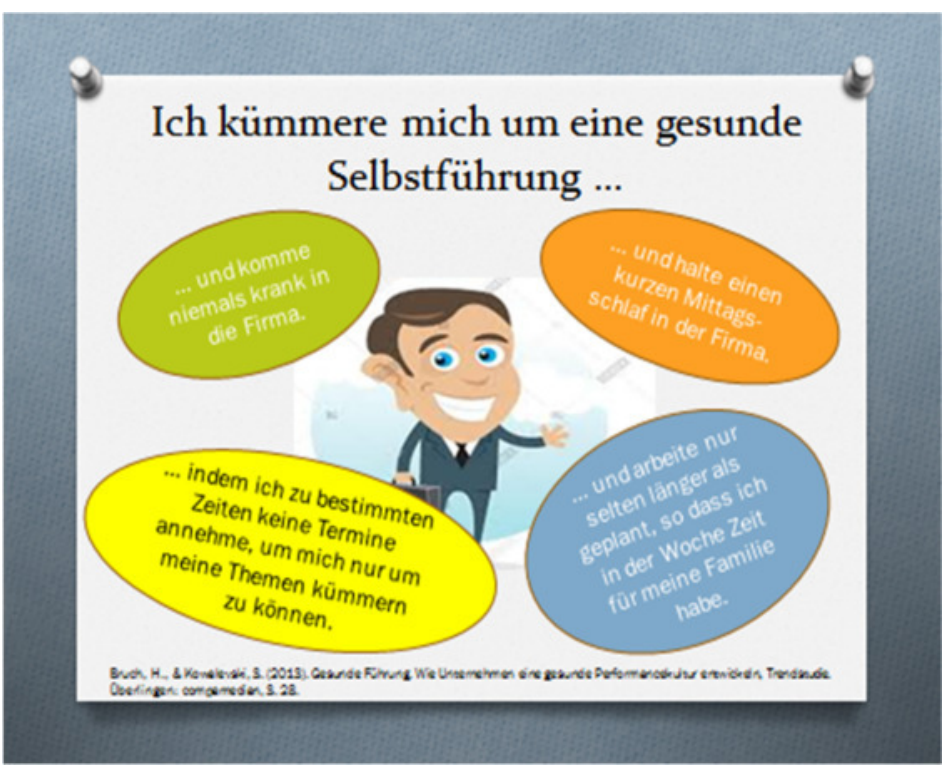

\section{Top Management:}

... Dieses Thema „ich komme niemals krank in die Firma“ heißt ja erst mal, man soll sich bemühen, auch als Inhaber, als Geschäftsführer nicht krank zu sein, so, das ist so okay.

...Wenn ich Diabetes habe, bin ich krank, aber nicht immer arbeitsunfähig. Das ist denke ich auch, wo man immer wieder in Selbstverantwortung über sich selber nachdenken sollte.

...Gerade Burnout ist sicherlich eine Erkrankung, die heute sehr massiv vorkommt, aber es wird auch schnell genannt......... Also es sind alles so Sachen, wo man denke ich auch ein bisschen vorsichtig sein muss, um nicht was zu suggerieren, was vielleicht gar nicht in der Welt da ist, auch dass die Leute es nicht so wollen. 
...Arbeitsverdichtung durch Arbeitsverkürzung ist ein Thema, muss man ganz klar sagen. Also heute muss in 35 Stunden das Gleiche geschafft werden, was früher in 40 oder in 45 Stunden gemacht wurde, und das hat zur Konsequenz, dass die Arbeit einfach verdichtet ist und dann diese Zyklen da kleiner sind, das muss man einfach wissen. Und damit ist ein Teil auch dieser Gesundheitserhaltung eben ins Private gerutscht, wird aber heute nicht mehr so wahrgenommen. Also interessant ist, es gibt ja immer diese Wellenbewegungen, vielleicht haben Sie recht: Wir haben gerade so eine Wellenbewegung, wo die Welle wieder rückwärts ist, back to the sixties, wo ein paar Aspekte aus den 60ern wieder hervorgekramt werden und dann geguckt wird, wie kann ich das jetzt in diese moderne Arbeitswelt integrieren.

... als Kind habe immer die Milchflaschen zurückgebracht.... das hat es alles gegeben, als die Welt noch in Ordnung war. Und dann ist sie so geworden, wie sie jetzt ist, was gar nicht unbedingt schlimm ist, aber es hat dann eben zu den Nachteilen geführt, die Pausen wurden verkürzt, weil die Leute gerne früher nach Hause wollten. Früher gab es ja nicht so viel Privatleben wie heute, da war die Firma, da war die Arbeit die Gemeinschaft...Das hat sich mehr verlagert ins Private. Und die Arbeitszeit ist ja auch kürzer geworden, und das hat dann zu diesen Veränderungen geführt. Erst mal ist ja alles ungesünder, weil es hektischer geworden ist, die Ruhe zu Mittag ist nicht mehr und was sonst damit zusammenhing. Also das gab es früher alles. Da waren vielleicht die Arbeitsbedingungen ungesünder, weil es nicht mal schnell Unterstützung gab, aber ich glaube wir müssen ja auch unterscheiden ein bisschen zwischen Handwerk und Industrie.

...ein Inhaber von einem befreundeten Elektrounternehmen, macht jeden Mittag eine halbe Stunde - und der pennt auch

...Das machen doch die Mitarbeiter dann erst recht und in Potenz, wenn einer sagt, "Privat geht vor geschäftlich“, das muss man den Mitarbeitern gar nicht erst beibringen, das haben manche sowieso schon verinnerlicht. Also wir müssen doch ein Vorbild sein, natürlich haben wir ein Privatleben, aber das darf nicht ins Geschäftliche - finde ich persönlich, vielleicht bin ich auch altmodisch,

...natürlich kann jeder seine Mittagspause nutzen wie er will. Der eine raucht, der andere schläft. Und wann man dafür Betten, einen Schlafsaal zur Verfügung stellen müsste, weil da die Nachfrage ist, ich glaube da hätten wir alle nichts dagegen.

...Und wenn ich mir meine Kollegen von den ganzen Internetfirmen angucke, die haben zeitlich noch ein ganz anderes Pensum als unsere Leute, die eine 35-Stunden-Woche haben, weil die kloppen da in der Tat dann 60 Stunden oder wie auch immer und nehmen das nicht als Arbeit wahr, weil dann setzen sie sich mal zusammen, diskutieren eine Stunde, essen schön, und dann geht es aber weiter.

...das komische ist ja, wenn wir an die 60er Jahre zurückdenken, was ich auch nicht kann, ich kann es wohl gerade noch, aber da waren bei uns anderthalb Stunden Mittagspause in der Firma, und die Leute gingen nach Hause und aßen mit der Familie und nahmen vielleicht noch einen halbe Stunde Mittag und dann kamen sie wieder.

... wir müssen glaube ich so ein bisschen wegkommen von dem Thema "das eine ist Gesundheit und Freizeit und das andere ist Arbeit." Wir alle arbeiten die meiste Zeit 
unseres Unternehmens im Unternehmen, und wenn ich mich da nicht wohl und gesund fühle und Spaß habe, dann mache ich was falsch. So, und ich darf dann jetzt nicht quasi die Zeit zu Hause, wo ich dann meinen Mittagsschlaf halte, dazu sagen, „Das ist jetzt meine Gesund-Zeit", sondern wir arbeiten heute einfach anders. So ist das Punkt.

\section{Mitarbeiter:}

...Ständige Erreichbarkeit.....Hohe Flexibilität, wird es immer schön genannt. Die Aspekte kommen dann wahrscheinlich eher durch als wie Sie schon sagten das positive andere Empfinden, dass er sich vielleicht um seine Gesundheit kümmert.

.. Man hat selber ein positiveres Gefühl, wenn man eigentlich Negatives tut, indem man ständig mehr arbeitet und auch mal mit der Grippe oder mit einem Husten zur Arbeit kommt und wenn es einem nicht so gut geht. Da fühlt man sich eigentlich besser, wenn man es macht als wenn man es nicht machen würde.

... da sieht man ja, wo der Trend hingeht, dass man sich da so unter Druck setzen lässt und dann „Jetzt bin ich schon wieder krank, jetzt kann ich nicht zu Hause bleiben, weil ich habe vielleicht Angst um meinen Job oder dass es vielleicht mal andere Maßnahmen hat."

... Wir arbeiten in der Firma in relativ kleinen Teams, und wenn bei vier oder fünf Leuten einer fehlt, dann sind das gleich 20\%/25\% Manpower, die dann weg sind und man hat was heißt ein schlechtes Gewissen, aber man macht sich dann seine Gedanken, und dann geht man doch lieber zur Arbeit.

\section{Slide}

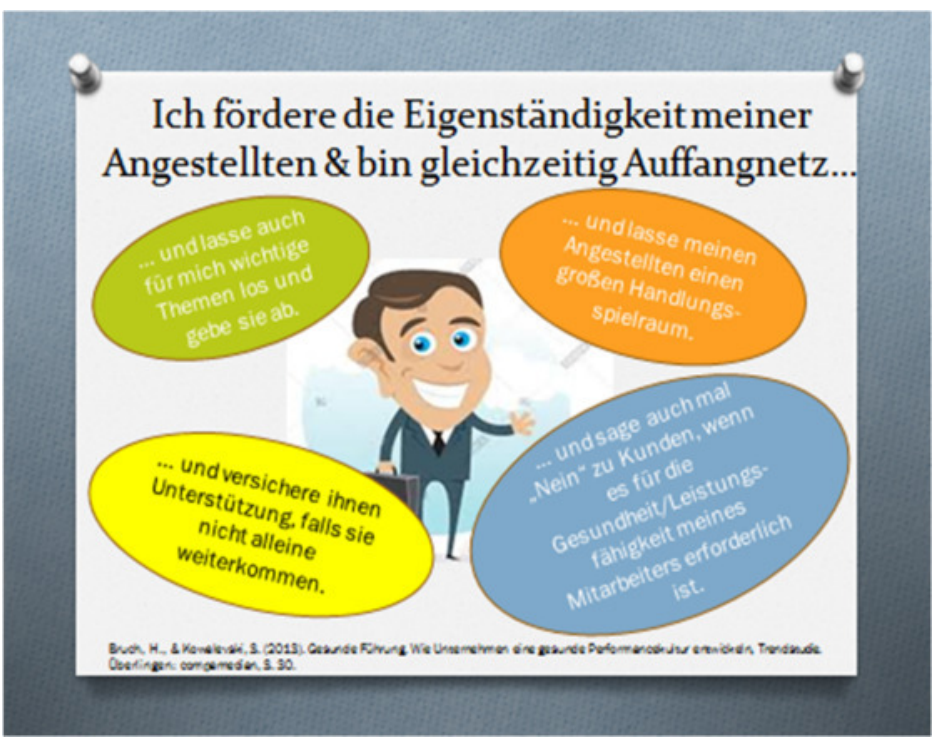

\section{Top Management:}

....die Leute schulen muss, dass sie sich nicht wehtun, dass sie mit dem Rücken gerade stehen, oder wenn sie was hochheben oder was auch immer, worauf man achten muss, 
dass sie sich richtig anziehen, wenn sie rausgehen und nicht gleich Schnupfen kriegen, weil sie ja vielen anderen Belastungen ausgesetzt sind.

... Die Verantwortung des Unternehmens für die Gesundheit, ich sehe die äußerst begrenzt.

...wir haben das Thema, das Unternehmen zahlt das, „Moment mal, brauchen wir doch gar nicht, eigentlich zahlt es ja die Krankenkasse." Rückenschule wird von der Krankenkasse übernommen. Da muss schon viel dazu kommen, wenn es nicht so ist, aber deswegen: Eigenverantwortlichkeit der Mitarbeiter.

... wir können nicht alle Bausteine, Rückenschule und Entspannungsmassage usw. im Unternehmen anbieten. Was Sie machen, ist ja genau richtig. Sie machen ja eine Art Feedback-Kultur: Sie sagen, „Achtung, du isst ein ungesundes Frühstück“, oder, „Ich finde es toll, dass du was machst." Also dieses Thema Gesundheitskultur findet eigentlich maßgeblich $\mathrm{zu} 50 \%$ an und wird abgedeckt durch eine Art Feed-back-Kultur und Ansprache. Es hat was ein bisschen mit Vorbild zu tun, aber auch, dass man Dinge anspricht.

...unsere Mitarbeiter haben freien Handlungsspielraum und ich gucke natürlich nicht wie die die Sachen auf dem Dach tragen, sondern hoffe, dass die das richtig machen in Bezug auf die Gesundheit.

..Alle anderen Sachen würde ich hundertprozentig unterschreiben, weil das genau eigentlich die typische Unternehmensphilosophie des Mittelstandes ist, was früher der Patriarch war, ist heute derjenige, der führt, mit Handlungsspielraum, mitnehmen, Partizipation an Entscheidungen und Delegation von Verantwortung.

...Aber informieren kann man trotzdem, Mitarbeiter, Kollegen, auch unter Kollegen sich austauschen, was fördert die Krankenkasse, was kann man durch so ein Bonusheft beispielsweise erschlagen? Das ist einfach wichtig. Das kann natürlich vom Betrieb ein bisschen gefördert oder ein bisschen aufgezeigt werden, „Die und die Möglichkeiten gibt es."

...dieses Thema Gesundheit, das muss anders gelöst werden, aber nicht dadurch, dass man sagt, wir sind übrigens jetzt drei Wochen geschlossen, wir haben jetzt mal so ein Sabbatical in der Firma mal eingeführt...

...Durcharbeiten ist ja auch nicht gesundheitsgefährdend, wenn es einmal am Wochenende ist oder so. Das ist ja nicht damit gemeint, das Beispiel, aber bei diesem Geschäftsführer, den wir 2047 ganz bestimmt im Unternehmen haben werden, weil der die falschen Einstellungen hat, der müsste doch nicht das sagen, sondern, der muss das doch lösen, das Problem, dass er ohne die Gesundheit seiner Mitarbeiter zu gefährden, dem Kunden seinen Wunsch erfüllt, das ist ja die Aufgabe des Managements.

\section{Mitarbeiter:}

.....wir arbeiten ja viel draußen, im Staub usw., sind viel auf den Dächern, d.h. Gerüsten usw. Wir arbeiten viel ohne, weil einfach kein Gerüst gestellt wird. Das ist ein großes Problem bei uns.......Ist auch schon oft diskutiert worden bei uns, aber dann heißt es immer, „Dann kriegen wir keine Angebote mehr." 
....ich denke mal, dass man schon alle Aufträge auch annehmen will um ein gewisses Umsatzziel zu erreichen, und dabei spielt es dann glaube ich nicht unbedingt die Rolle, ob ich da bei irgendjemandem was aufs Spiel setze.

... Nein" zu einem Kundenauftrag habe ich eigentlich selten erlebt. Nein, da möchte man einfach alles mitnehmen und keine Kunden an die Konkurrenz verlieren.

...Da kommt ja auch wieder das Argument, „Ja, wenn wir mehr Aufträge haben, haben wir ja auch mehr Geld, und dann haben ja unsere Mitarbeiter da auch was von, die Firma existiert ja länger." Ja, die Prioritäten sind da irgendwie anders gesetzt

\section{Slide}

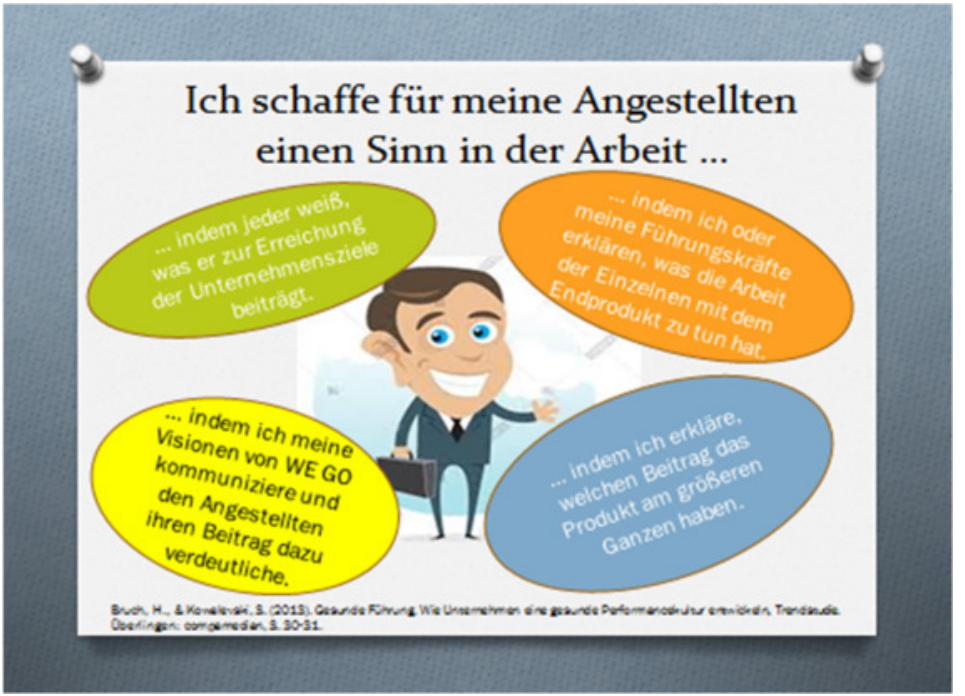

\section{Top Management:}

...Also dieses Thema Wahrnehmung, welchen Beitrag leiste ich, ist ein ganz relevantes. Und die Arbeitsteilung geht ja immer noch weiter. Das war vor 100 Jahren noch ganz anders. Da hat der Mitarbeiter ja quasi sein Endprodukt - gut bei Ihnen gilt das immer noch, aber bei uns hat er vor 100 Jahren ein Endprodukt noch gesehen. Und heute ist er nur ein Rädchen im Getriebe, und insofern muss man den auch da mitnehmen.

....Also Kommunikation, ja, wir sind uns total einig, aber gestern hatten wir auch den Fall mit einem Mitarbeiter aus der EDV, ich erzähle dem was, Reaktion: „Man hört aber auch immer alles als letzter, und nie informiert ihr mich." Er war der erste, der es erzählt kriegte, nun hat er aber auch den Eindruck irgendwie, weil, weiß ich auch nicht, weil die Entscheidung vorbereitet war und er da nicht auch noch dran mitwirken durfte, dass er nicht eingebunden war. Also manchmal ist es auch, man macht es nach dem Lehrbuch und es ist trotzdem nicht richtig. Aber das sind Menschen, so sind wir, jeder von uns hat ja seine Macken.

...Ich kenne keine Firma, wo das Thema Kommunikation nicht irgendwann wieder Thema ist. Es wird schlecht kommuniziert. Wenn man sich fragt, was ist schlechte 
Kommunikation? Ist es eine Bring- oder eine Holschuld? Also da bin ich auch sehr differenziert.

....Dieser mündige Mitarbeiter, der da immer postuliert wird, den gibt es, aber es gibt auch eine ganze Menge Leute, die wollen das gar nicht sein, die wollen gesagt kriegen, was sie tun sollen, die wollen eingebettet sein in ihre Routine. Die sind gar nicht glücklich über so viel Eigenständigkeit.

...Aber wir sind ja 2046 und ich bin ganz überzeugt, dieser Anspruch, der steigt. Also wirklich, natürlich haben Sie Leute, das darf man auch nicht unterschätzen, und es gibt auch ganz monotone Arbeiten, die Leuten kommen hin, wollen ihr Geld abholen und dann - in Anführungsstrichen - „ihre Ruhe haben.“ Auch das gibt es.

\section{Mitarbeiter:}

....Bei interessierten Mitarbeitern, da sage ich, die haben einen feineren Job. Wenn ich einen habe, der täglich nur Null-acht-fünfzehn macht, das bringt mir nichts. Der kommt morgens rein, geht nachmittags nach Hause, der identifiziert sich praktisch nicht mit dem, was man macht.

...Wenn die Kommunikation zwischen dem Chef und uns Mitarbeitern besser wäre, wird sich wahrscheinlich auch am Arbeitsverhalten einiges ändern.

...Man müsste vielleicht mehr eingebunden werden an den Baustellen. Dass man den Tag vorher Sachen noch nicht hat, bei Großbaustellen, da kriegt man morgens, da haben Sie eine halbe Stunde Zeit sich da reinzudenken und dann geht es sofort los. Und was ist dann mit "Wie mache ich es am besten“? Da hat man gar keine Chance selber vielleicht noch mitzudenken und Ideen mit einzubringen, was man besser machen könnte, das ist viel zu kurzfristig.

...im Bekanntenkreis kenne ich jemanden, der arbeitet zum Beispiel in der mobilen Pflege für alte Leute, da sehe ich zum Beispiel persönlich viel mehr Sinn drin, er macht viel mehr für Menschen als jetzt hier im Büro. Da scheffelt man ja in die Taschen vom Chef quasi. Solchen Sinn und solchen Sinn gibt es, aber es stärkt ja auch das Wir-Gefühl zu sagen, wenn wir alle hier gut zusammenarbeiten, dann erreichen wir am meisten und dafür muss dann das auch passen, der Zusammenhalt, und man muss wissen, wofür man es dann tut, klar.

...Das finde ich schon gut, dass ich das Wissen auf jeden Fall mitgenommen habe, wie alles funktioniert. Wenn neue Mitarbeiter kommen, die kennen ja die Abteilungen nicht, die kennen ja nur ihren Arbeitsplatz und wissen noch viel weniger........also das ist wichtig, auch mal andere Abteilungen zu sehen, einfach um das große Ganze besser zu verstehen.

...Als ich damals bei XXX angefangen hatte, habe ich darauf gedrängt, den Betrieb auch kennenzulernen, und dann wurde mir auch die Möglichkeit gegeben, dass ich eine Woche im Betrieb sein durfte, weil mir das ganz wichtig war, alle Abläufe kennenzulernen, ja, was mir dann natürlich hinterher in meiner täglichen Arbeit sehr viel weiter hilft.

....nur wenn er da drüben ist und sieht die Probleme, die die damit haben, dann hat der auch eine ganz andere Wahrnehmung für diese Geschichte. Und wir haben das glaube ich vor zehn Jahren schon einmal angeboten, da haben wir den Mitarbeiten angeboten, mal 
einen Kunden zu besuchen, dann haben wir zwei Autos vollgepackt, dann konnten die die Zigarettenproduktion angucken....... Die sagen, „Mensch, ich mache das Produkt und ich möchte ganz gerne mal sehen, wie jetzt die Schachte, die ich produziere, weiterverarbeitet wird. Die können sich da gar nichts drunter vorstellen, mit was für Kontakten das da weiterverarbeitet wird, wie das da alles ineinander spielt.

...Muss öfter mal ein Feedback zurückkommen vom Chef, dass man mal was hört.

....Grundsätzlich ist es ja so, das Feedback, was man meistens bekommt, ist ja erst mal ein Negatives, wenn man irgendwo ein Problem gehabt hat, was zu Kundenreklamationen geführt hat oder sonst irgendwas. Der Mitarbeiter braucht aber ein positives Feedback. Und wenn der dann nur Negatives hört, dann geht die Laune auch in den Keller. Und wenn der überhaupt kein Feedback hört, dann kommt er sich eh überflüssig vor. Also diese Feedback-Geschichte ist doch schon wichtig.

....Das schafft auch eine gute Kommunikationsbasis, wenn man sich nur einmal im Jahr wirklich zusammensetzt - umso öfter man miteinander spricht, umso einfacher fällt es einem vielleicht auch Themen anzusprechen, die man in diesem Gespräch, was einmal im Jahr stattfindet, sich nicht trauen würde vielleicht auch anzusprechen.

...Also wenn man keinen Sinn in seiner Arbeit sieht, das wäre eigentlich das Schlechteste, der schlechteste Ausgangspunkt. Und wenn der Chef oder der Vorgesetzte auch kommuniziert, was man selber für das Unternehmen leistet, wohin der Beitrag, den ich leiste, wo der eingeordnet wird, dann wäre das auf jeden Fall eine gute Sache.

...genau, beim Jahresgespräch, aber es müsste häufiger stattfinden.

\section{Slide}

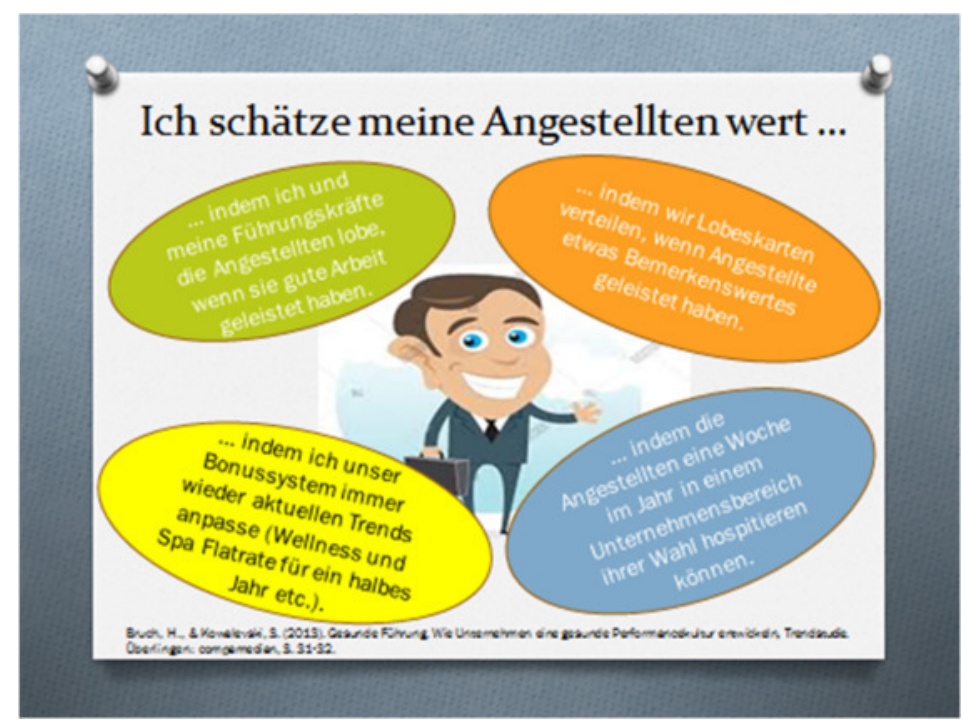




\section{Top Management:}

...Wellness- und Spa-Flatrate können wir uns nicht leisten, egal wie gut das für die Gesundheit der Mitarbeiter ist.

... das halte ich für sehr kostspielig.

... die Anspruchshaltung ist da dann auch sehr schnell groß. Die muss man dann erfüllen.

...Ich glaube aber auch, dass solche Sachen, so regelmäßige Bonussysteme oder so, die verpuffen dann auch irgendwann. .......aber ich glaube so regelmäßige Sachen, die schleifen sich dann auch relativ schnell ab. Oder man müsste an verschiedenen - also in der Kantine mal das machen, oder Hautschutz oder irgendwie in der Richtung.

...Wenn ich zwei Vorsorgeuntersuchungen gemacht habe, kann ich mir dafür den eigentlichen Eigenanteil auch wiederholen dann bei meiner Kasse, zum Beispiel. Und hake ich dann bei meiner Krankenkasse vier Punkte ab, kriege ich dann vielleicht 100 Euro am Jahresende wieder..........aber man kann, je nachdem, was man so macht, wie die Vorbildfunktion des Unternehmers ist.

... warum ist es denn eigentlich, dass in Amerika employee of the month funktioniert und bei uns nicht. Eine ganz andere Kultur, die Amis sind da anders. Amerikanische Mitarbeiter fühlen sich im täglichen Wettbewerb mit allem und jedem. .......ch habe eine Zeitlang da drüben sein dürfen, das ist der Hammer, wie die immer überlegen, wie kann ich jetzt noch einen Schritt besser werden. Ob für mich oder für das Unternehmen ist egal, aber immer im Wettbewerb. Immer mit einer gewissen Anspannung. Und gerade in Ostwestfalen halte ich das für schwierig.

......Einer der größten Punkte, die von den Mitarbeitern sehr früh erwähnt wurden, was sie wünschen würden, was künftig anders ist, dass man auch mal ein Feed-back bekommt oder ein Lob, „Das hast du gut gemacht“.........Das ist wichtig für die Leute. Das ist mir in dem Moment sehr bewusst geworden.

...Lobeskarten, ja, weiß ich nicht, employee of the month haben wir nicht. Ich hätte auch immer Angst davor, dass alle anderen sich dadurch zurückgestellt fühlen, weil wenn einer dreimal hintereinander „Angestellter des Monats“ ist oder er das Glück gehabt hat, dass er das gut gemacht hat - ich finde das schwierig den anderen gegenüber, weil wir dann auch sehr ähnliche Tätigkeiten haben.

.... Wir sind ja in Ostwestfalen, das ist ja ähnlich wie in Süddeutschland, „Nicht geschimpft ist genug gelobt", das hört sich so ein bisschen so an - aber Feed-back heißt für mich Rückmeldung im Guten wie im Schlechten.......da hat sich in den letzten Jahren ganz dramatisch was geändert in den letzten zwei Jahrzehnten. Und das erwarten die Mitarbeiter.

.... es mir persönlich manchmal schwer, den zu loben, wo er mich gerade geärgert hat. Und dann ärgert er mich zweimal und dann lobe ich ihn schon gar nicht mehr. 
....Ich meine ja. Man kann sich die Leute ja nicht schnitzen und jeder hat seine Fähigkeiten und Stärken und auch seine Schwächen. Und unsere Aufgabe als Unternehmer ist ja immer, einmal das zu beurteilen, zu gucken: Ist die Stärke so wichtig und gut für das Unternehmen, dass er genug PS auf dem Unternehmen einen Wert zu geben. So. Nur dann funktioniert das. Und wenn die Schwäche, sprich die Beule im Auto, Überhand nimmt, dann muss man eine andere Maßnahme finden. Aber es gibt Mitarbeiter, die sind in einzelnen Bereichen sehr, sehr stark, also unser Versuch ist immer Stärken stärken und Schwächen schwächen. Das ist die alte Regel, aber das kriegen sich auch nicht immer hin. .... Wir haben die Betriebsverfassungen, wir haben Kündigungsschutz, diese ganzen rechtlichen Rahmenbedingungen, das sind ja ganz andere Dinge. Die Mitarbeiter sind ja sehr geschützt. Das ist auf der einen Seite gut, manchmal natürlich furchtbar hinderlich für das Unternehmen, weil Mitarbeiter sehr genau ihre Rechte kennen, manchmal besser als ihre Pflichten, und gerade bei Langzeitkranken ist es halt so, da kennt man recht schnell Gesetzeslücken und wie man sich von der einen Kasse in die nächste rettet, also das kriegt man relativ schnell spitz.......

\section{Mitarbeiter:}

....das ist ja auch das, wie man sowas rüber bringt, so ein Feedback. Ich hatte mal einen Mitarbeiter dabei, der hat mir nachher gesagt, „Ich verstehe, was du sagst, aber ich weiß nicht, was du meinst", weil ich das dann wahrscheinlich zu sehr verpackt habe. Ich habe das dann in ganz schmale Worte verpackt, wo ich weiß, "Aha, die versteht er", auch mit einem ganz anderen Slang. Und dann war alles in Ordnung. Jetzt weiß ich es halt. Perfekt...auf das "Wie“ kommt es an.

.....Ich glaube schon, dass man wertgeschätzt wird, nur es kommt von oben nicht so rüber. Von den Kunden kommt da mehr......Wäre gut, wenn da von oben mal ein bisschen mehr kommt, wenn die Kundenfirma angerufen hat, wir Erfolg haben und es ist alles in Ordnung.

....Muss auch einfach sein zwischendurch, zu sagen, „Mensch, hast du gut gemacht", oder „Toll, freue ich mich.“ Es kommt nur manchmal einfach zu wenig......

..... Negatives Feedback kriegt man sofort. Und das Positive, na ja, das bleibt so ein bisschen auf der Strecke.

..... am Ende des Tages ist man immer nur eine Nummer in dem Unternehmen. Und ob dann immer mein Einsatz auch letztendlich in dem Sinne wertgeschätzt wird? .... ich motiviere mich immer sehr viel selber......

......Ich kann mich schon selber motivieren und ich fühle mich auch wertgeschätzt, aber ich habe auch gemerkt als Führungskraft, nicht jeder Mitarbeiter kann mit einer Wertschätzung umgehen.

....Es kommen dann auch schon mal Fragen so wie, „Das Lob, das du mir jetzt gegeben hast, meinst du das jetzt wirklich ernst oder willst du mich jetzt verarschen?" .... es wird wohl auch teilweise sehr selten gemacht......und wenn es dann nicht kommt, in dem Augenblick, wo ich dann auf einen Mitarbeiter zugehe, dann sagt der, „Moment mal, das 
habt ihr die letzten zehn Jahre nicht gemacht, warum fängt das jetzt auf einmal an?“ So, "Irgendwas willst du doch von mir."

...einfach ein ganz normales Danke, das finde ich einfach schon das Netteste. Und es ist ja auch blöd, wenn du dich durch Lob veräppelt fühlt, so, „Wie meint er das jetzt, hat der das vielleicht ironisch gemeint?", wirklich, es heißt ja auch immer, jeder ist ersetzbar und das wird auch, kann auch knallhart durchgezogen werden. Es suchen ja genug Leute Arbeit, und wenn einer nicht wirklich mitspielen möchte, dann wird er ausgetauscht. Ist halt so.

... ich finde Wertschätzung immer noch einer der wichtigsten Punkt ....ich würde für mich eher sagen eine vernünftige Kultur der Wertschätzung schaffen und um diese Kultur herum dann diese Geschichte mit dem ganzheitlichen Gesundheitssystem aufbauen. Der Rest kommt, wenn das vernünftig läuft. Und für die Arbeit fordern und fördern, das kommt meiner Ansicht nach alles so selbständig hinterher. Zufriedene Mitarbeiter und das Gesundheitssystem aufbauen und dann Sinn für die Arbeit und alles, was da steht, sind dann positive Nebeneffekte, würde ich sagen.

...Wertschätzung und Förderung, also wäre mir ....schon wichtig.

.... so einen Bonus fände ich auch klasse, wenn man sagt, „Wir beteiligen uns vielleicht für ein Abo in dem Fitness-Studio nebenan, oder in dem Wellness-Bereich, oder wir unterstützen das ein bisschen",

....Dieses Bonussystem, das haben wir ja schon im Hause, da muss der Mitarbeiter, wenn er 52-mal im Jahr ins Fitness-Studio geht, dann bekommt er einen gewissen Beitrag dabei...das wird von den Mitarbeitern ganz gut angenommen.

\section{Slide}

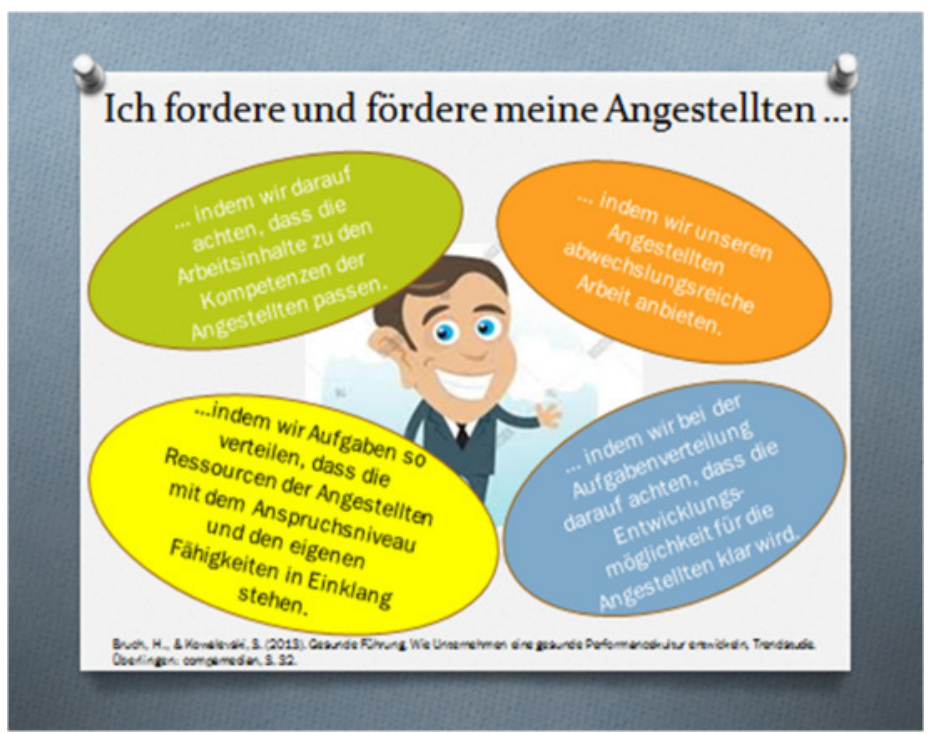




\section{Top Management:}

... Gesundheitsmanagement zu fokussieren nur auf das Thema Rückenschule oder nur auf E-Bike oder Flatrate im Sportcenter, das ist zu wenig, also ich würde das Thema in der Tat viel globaler sehen.

...das Thema fängt bei mir ... schon bei Unternehmenskultur an, einer wertschätzenden Feedbackkultur, positiv wie negativ, also dieser ganze Ring der Maßnahmen und was da rein spielt, und den Mitarbeiter nicht überfordern. Ihn fordern und fördern, aufpassen, dass das fordern nicht ins überfordern kommt; dann kommt eben auch Krankheit, Burn out, was auch immer.

.....ich würde mir wünschen, dass so ein Thema Gesundheitsmanagement, ein Baustein immer genannt oder ungenannt auch in so einer Unternehmensstruktur ist, dass man das implizit mit reinnimmt und sagt: „Unsere Unternehmensmission ist...."

....Unternehmenskultur wird immer ganz stark geprägt durch - wir sind hier ja Mittelstand - durch den Unternehmer, durch Sie persönlich, durch Sie als Unternehmerfamilie, aber Unternehmenskultur, sage ich allen Mitarbeitern und Mitarbeiterinnen, das sind wir alle, d.h. also jeder ist Baustein, aber Prägung, so diese DNA, wird natürlich auch durch den Unternehmer, die Unternehmerfamilie gelebt, das muss ich schon sehen.

...Wenn ich da eine Sache nur ergänzen darf....., dass man es nicht nur so im Mikrokosmos Gesundheit diskutiert: Wir machen seit Anfang dieses Jahres zusammen mit dem Inhaber XXXX jeden Montag mit acht Führungskreisrunden eine sogenannte „....Entwicklungs- und Koordinationsgespräche", das sind 3-Stunden-Gespräche. .....Wir müssen die Leute auch mitnehmen, und da ist auch dieses Thema Gesundheit einer der Punkte...... Und wenn man das dann quasi in so einem Instrument einfügt und da mit Gesundheit eine Rolle hat, dann hat man glaube ich den Automatismus, wo das dann auch so eine Art Baustein der Unternehmenskultur am Ende sein kann.

\section{Mitarbeiter:}

....Da würde ich sagen wissen die Chefs nicht so genau, wer im Team welche Aufgaben überhaupt übernimmt und welche Stärken oder Schwächen da vorhanden sind. Da geht es nur darum, dass das Gesamte am Ende stimmt und wer was davon leistet würde ich sagen, ist den Chefs nicht so bewusst.

...da muss man wirklich unsere Chefs auch loben, dass die sich schon für jeden Mitarbeiter interessieren, unser Chef kennt auch die Namen von jedem XXX, in der XXX, also da ist er wirklich sehr bemüht auch, den persönlichen Kontakt zu halten und mit jedem auch zu sprechen. Das ist nicht nur, "Ich sitze oben in meinem kleinen Schlösschen, und Hauptsache die Ergebnisse stimmen", so ist es auch nicht bei uns. Schon ein typisches Familienunternehmen.

...das weiß der Chef natürlich auch, und dann weiß er schon, wer macht gerne was und dann wird das auch so aufgeteilt, dass wir da Spaß an der Arbeit haben. 
...Man braucht halt einen direkten Kontakt zu Chef.

... Fördern und fordern. Ja, einfach auch einzeln auf Mitarbeiter eingehen, die wirklich kennen und deren Pros und Cons halt kennen und damit arbeiten, finde ich eigentlich eines der wichtigsten Sachen.

... Da müsste vielleicht vorher noch ein Feedback kommen, bevor man beim Kunden steht.

...Dass jeder Mitarbeiter das tut, was er auch kann; und wenn nicht, dann fährt er bei jemandem mit, der es gut kann, und dass er das mitkriegt, und das nicht alleine macht. Das geht bei uns gut.

....ich denke schon, dass das schon wichtig ist,....dass man eine Chance hat, sich weiterzuentwickeln. Viele wollen ja dann auch mal andere Jobs übernehmen. Also die Möglichkeit sollte grundsätzlich gegeben werden, vielleicht auch zusätzliche Schulungen besuchen.

... Ich weiß auch Mitarbeiter, die mit dem, was sie machen zufrieden sind und gar nicht was Neues lernen wollen. Also gibt es bei uns auch wieder. Also die wollen gar keine anderen oder neuen Aufgaben übernehmen und sind zufrieden, so wie es ist.

\section{Slide}

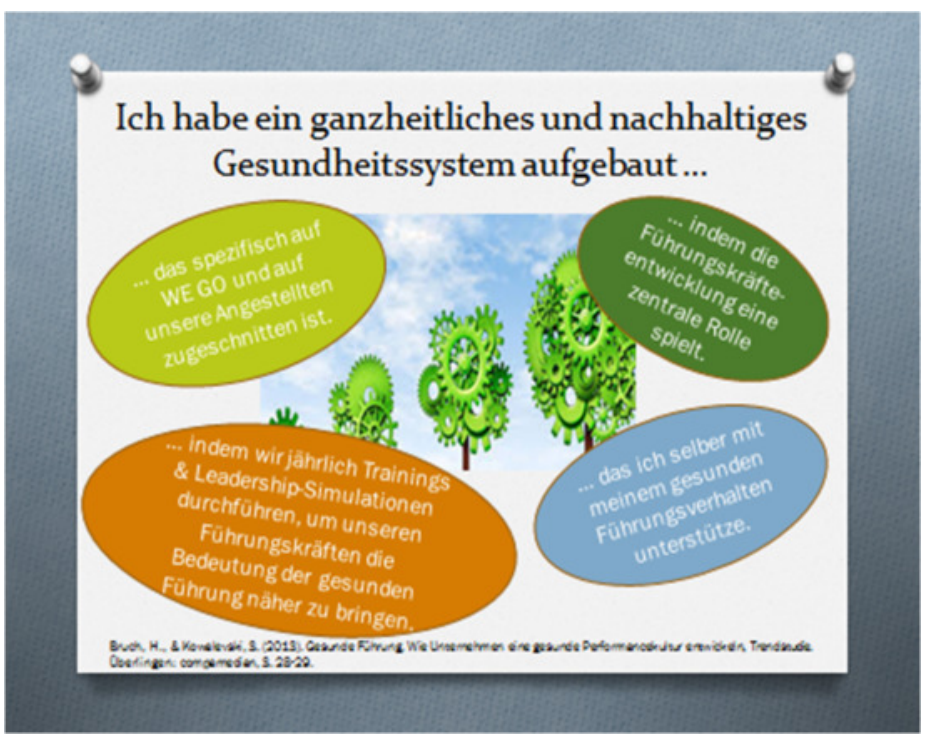

\section{Top Management:}

...nicht nur der Inhaber führt durch Vorbild, sondern er braucht ja auch Multiplikatoren. Das steckt ja hinter diesem Thema Führungskräfteentwicklung, wie das quasi bis in alle Ecken des Unternehmens getragen wird. 
.... also wo wir zum Beispiel gesagt haben, wir schulen unsere Schichtleiter mal in dem Thema Krankenrückkehrgespräche, wie führe ich so ein Thema wertschätzend, nicht nach dem Motto, "Na, bist du endlich wieder da? Warum warst du vier Wochen krank?", sondern eher das Thema, „Schön, dass du wieder da bist“, und „Alles wieder gut? Können wir dir was tun?“, usw. Das Thema wertschätzend - aber auch immer mit dem Hinweis, „Ja, aber es sind auch alle hier zum arbeiten da."

...ich denke das sind so Dinge, die künftig noch mehr eine Rolle spielen im Sinne einer Systematik, Sie haben es ganzheitlich genannt, also sprich, „Das darf nicht verpuffen als Einmalmaßnahme." Das war bei uns in der Vergangenheit auch so oft: Wir haben so eine Maßnahme gestartet, und dann drei Jahre ruht das, das ist nicht nachhaltig. Da muss man also immer dran bleiben.

...ich glaube was auch sehr, sehr typisch ist, dass die Unternehmer, die ich jetzt selber kennengelernt habe, sehr nah am Mitarbeiter sind. Also das sind Unternehmer zum anfassen, die kennt man, man kennt das Gesicht, sie sagen auch mal "Guten Tag“.....aber er kann natürlich nicht alle 400 Mitarbeiter tagtäglich sprechen, das funktioniert natürlich nicht.

....Was wir festgestellt haben, was bei uns - das geht so ein bisschen Richtung feel good, aber eben dann auch Richtung Bewegung und das Überbringen von verschiedenen Möglichkeiten gibt es auch noch, was zu tun ist: Gemeinsame Veranstaltungen zu machen. Ich bin eigentlich kein Freund davon, Arbeit und Privates zu vermischen. Was wir aber festgestellt haben ist, dass wir bessere Stimmung im Unternehmen haben, wenn wir regelmäßig gemeinsam was tun.....

...und ja, auch von meiner Rolle her vermehrt Vorstellungsgespräche auch vom Helferbereich jetzt geführt, um halt zu sagen, ja, das fängt schon beim Helfer an und nicht erst bei der Sachbearbeitung, wo halt Wertschätzung, Führungsrolle, Unternehmenskultur vermittelt wird.

\section{Mitarbeiter:}

.....Ich finde, dass es bisher eigentlich noch viel zu wenig Klick gemacht hat im Kopf der Führungskräfte, weil irgendeiner muss davon auch absolut überzeugt sein, dass das wichtig ist, in seine Mitarbeiter und deren Gesundheit zu investieren, und ich finde, dass das Thema einfach noch nicht wichtig genug ist.....

...das finde ich ganz wichtig, dass natürlich erst mal die Führungskräfte mitgezogen werden, weil wie wir vorhin auch schon gesagt haben, die einzelnen Angestellten sind ja nicht so nah direkt am Chef, sondern eher an ihren Abteilungsleitern, und wenn die das auch weiter kommunizieren oder für sich dann festgestellt haben, dann ist das für die näher als wenn der Chef das selber nur kommuniziert.

..wie schon gesagt, das muss auf jeden Angestellten selbst zugeschnitten sein. Wenn ich das bei uns sehe, so ein Drucker im 3-Schicht-Betrieb hat ganz andere Belange als ein kaufmännischer Angestellter..... 
... In erster Linie sehe ich ...., dass jeder für seine eigene Gesundheit zuständig ist. Auf der anderen Seite sollte eine Bereitschaft oder auch eine, ja, vom Chef auch irgendetwas kommen, also dass der Druck nicht größer wird wie zum Beispiel psychische Schäden, ...... dass man da auf die Mitarbeiter achtet, dass die nicht sich selber so unter Druck gesetzt fühlen und dann lieber krank zur Arbeit kommen, weil sie Angst haben, gekündigt zu werden, dass in der Hinsicht der Mensch ein bisschen mehr zählt .......

....man kann sich wahrscheinlich auch den Erfolg nicht vorstellen, bis man in nicht dann mal erlebt hat, das ist schwierig messbar...... und das hat man auch nicht nach sechs Monaten oder nach einem Jahr, das wird wahrscheinlich zwei oder drei Jahre dauern, bis man sehen kann, hier hat sich in der Belegschaft jetzt wirklich was positiv verändert.

...die Führungskräfte im Unternehmen zu schulen, was gesunde Führung bedeutet, das ist .....schon relativ wichtig.......

...hört sich in der Theorie auf jeden Fall sehr gut an.......ist halt die Frage, wie man das praktisch umsetzen kann.

....Ja, ist schwierig, jemanden zu überzeugen ein Programm wirklich anzufangen und durchzuziehen. Da muss schon eine große Bereitschaft vorhanden sein.

\section{Slide}

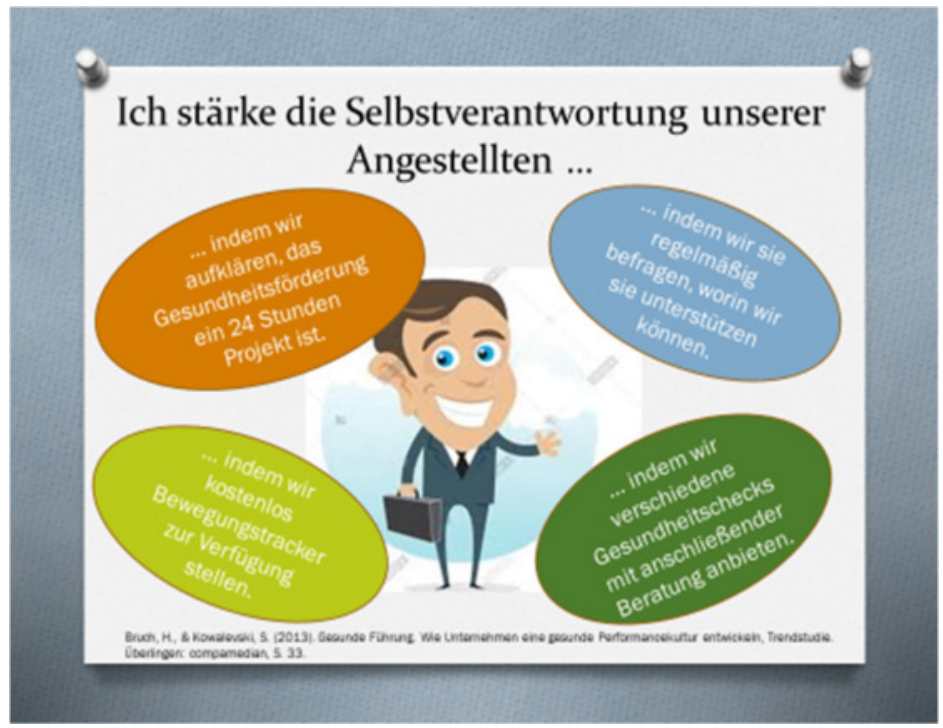

\section{Top Management:}

... das ganze Thema Datenströme und Gesundheitsbelt, ....., "Sehe ich kritisch", da sind noch eine Menge Fragen zu klären.

....dieses Thema "Intranet“ ist jetzt ja ganz toll gemacht. Ich glaube auch, dass die Sachen wachsen, aber auch wir bieten es an. Ich kann nur sagen, das ist eigentlich ein 
Datenfriedhof, wenn das nicht mit Inhalten gefüllt wird, was die Leute auch - wofür die brennen, dann nutzt das Thema Intranet niemand.

....Da betest du abends mit deinen Eltern und dann sagst du, was war denn schön am Tag und wen habe ich lieb. Und das war ja wunderbar. So, wer macht das von uns heute noch? Was haben wir als Kind alle gelernt? Der eine oder andere tut es vielleicht gelegentlich oder auch immer, aber die meisten tun es sicher nicht, ich jedenfalls nicht, und da sollen wir jeden Abend bevor wir nach Hause gehen ins Intranet gehen und schreiben, was ich noch nicht mal abends privat tue?

......also „Ich tue alles und ihr müsst nur konsumieren." Da fehlt mir irgendwie der Satz „Ich frage meine Mitarbeiter", nicht "Ich frage sie, worin ich sie unterstützen kann", sondern ich frage sie, was sie getan haben für ihre Gesundheit"

....Wir haben die Verantwortung der Mitarbeiter. Das ist das Entscheidende. Und alles, was an Gesundheitsförderung kommt als Angebot oder als Pflicht, wenn der Mitarbeiter selber das nicht für sich annimmt und sagt, ich bin für mich und meinen Körper verantwortlich, nicht nur mein Bauch gehört mir, sondern alles, solange können wir noch so viel anbieten, oder können wir noch so viel reden

.....Selbstverantwortung, da sind zwei Sachen in dem Begriff drinnen, das fängt mit selbst erst mal an.

.... wir können als Unternehmen einen begrenzten Beitrag, einen begrenzten Rahmen zum Thema Gesundheit beistellen, aber gefühlt der größere Teil ist das Thema Eigenverantwortung.....

....Wir hatten vor drei Jahren, ich glaube mit der DAK war es, einen Gesundheitstag der Mitarbeiter bei uns, ....... Von den 400 Mitarbeitern haben glaube ich 20 das wahrgenommen. .... und dann haben wir die Aktion noch mal gemacht und da war die Resonanz ähnlich. D.h. es wurde - da müssen wir uns selbst an die Brust fassen, warum nicht, aber es wurde nicht gewünscht, dieses Thema Gesundheitscheck. Also vielleicht ist es auch nicht Teil, da in dem Rahmen von der Unternehmenskultur gewesen.

.... ich denke es gibt verschiedene Bausteine, wo man an dem Thema Gesundheit bauen kann, aber da bin ich immer vorsichtig mit so Beispielen,...... Da gibt es andere, verschiedene, und da ist nichts richtig und nichts falsch darin.

.....Gesundheitscheck würde ich hinterfragen, den Bewegungstracker halte ich für Bullshit. Der bringt ja nichts.

....Wir werden ja überall beschützt. Und das lieben wir Deutschen. Also wenn uns einer eine Vorschrift macht, befolgen wir sie auch. Bei der Gesundheit werden keine Vorschriften gemacht, da wird ganz an Eigeninitiative appelliert. Vielleicht müsste da auch ein bisschen mehr Zwang rein. Egal, ob der nun von der Regierung kommt oder ob er von den Firmen kommt, oder gesellschaftlicher Druck ist auch schon mal was .....ob es vielleicht dann eben am Ende ein bisschen Regierungsaufgabe ist, irgendwas sich auszudenken, was sie sich in allen anderen Bereichen auch für uns ausdenken, was wirklich gut für uns ist. 
...... Ich glaube es ist wahrscheinlich ein politischer oder gesellschaftlicher Diskurs notwendig, um diese Erwartungshaltung auch noch mal in der Gesellschaft zu formulieren....

.....Selbstverantwortung, ..... und das ..... auch in so ein öffentliches Bewusstsein, auch politisch gerne zu stellen, das ist, denke ich, eine Aufgabe. Also die mischen sich überall rein -Aber bei solchen großen Sachen, da wird nicht mal was gesagt, da gibt es nicht mal eine öffentliche Meinung dazu. Die Presse diskutiert und haut drauf bei dem ganzen Thema Rauchen ...., aber gucken Sie mal, ob es einen Leitartikel in der FAZ oder so gibt zu diesem Thema Gesundheit und Selbstverantwortung. Das ist nicht sexy zurzeit.

......,Ich muss was für meine Gesundheit tun", das muss verankert werden, dafür müssen wir schulen, da müssen wir drüber reden, das tun ja auch die Krankenkassen, jeder redet darüber. Das dann vielleicht noch runterbrechen: Was heißt das für dich persönlich.

\section{Mitarbeiter:}

..... Mit den Gesundheitschecks, in der Firma, wo ich vorher war, da gab es einmal jährlich Gesundheitschecks. Da musste man hinkommen.

.....Wichtig ist ja nur, dass man dann auch gegensteuert, wenn man was feststellt, oder? Ja, und das nicht einfach so hinnimmt, sondern wenn man dann was feststellt, dass man dann auch sagt, „Ja, gut, dann müssen wir irgendwelche Arbeitsbedingungen ändern“, ich weiß nicht, bei uns irgendwas mit Farbe, keine Ahnung, ob man da was abstellen kann?

....Der Herr Müller wird als Kernkompetenz haben, seinen Krankenstand in der Firma so gering wie möglich zu halten. Das ist ja das Ziel, was er verfolgt.

...Wenn man es so von null auf hundert einführen würde, Gesundheitschecks usw. die Mitarbeiter vielleicht eher Angst hätten, dass sie irgendwie da kontrolliert werden, so, „Erst mal gucken, wen müssen wir denn hier aussortieren“.... das ist erst mal das erste, was ein Mitarbeiter im Kopf hat, wenn er zum Gesundheitscheck muss, „Oh, was wollen die jetzt von mir?."

....da würde man dem Arbeitgeber schon ein sehr großes Vertrauen entgegenbringen, denn wie will man die Ergebnisse von meinem Gesundheitscheck so ohne Einschränkung betrachten oder bewerten, das ist ja fast unmöglich.

...wenn der Chef einem sowas alles anbietet, dann kann ich mir schon vorstellen, dass das einen positiven Aspekt haben kann.

.... es hat ....auch sicherlich .... was mit Wertschätzung zu tun. Der eine sagt, „Ich habe einen Schnupfen und bleibe zu Hause, weil es interessiert hier ja sowieso keinen", und der andere sagt, selbst halbtot komme ich noch in die Firma.

.....dass man das mal ausprobiert. Nur die Zeit muss auch da sein. 


\section{Slide}

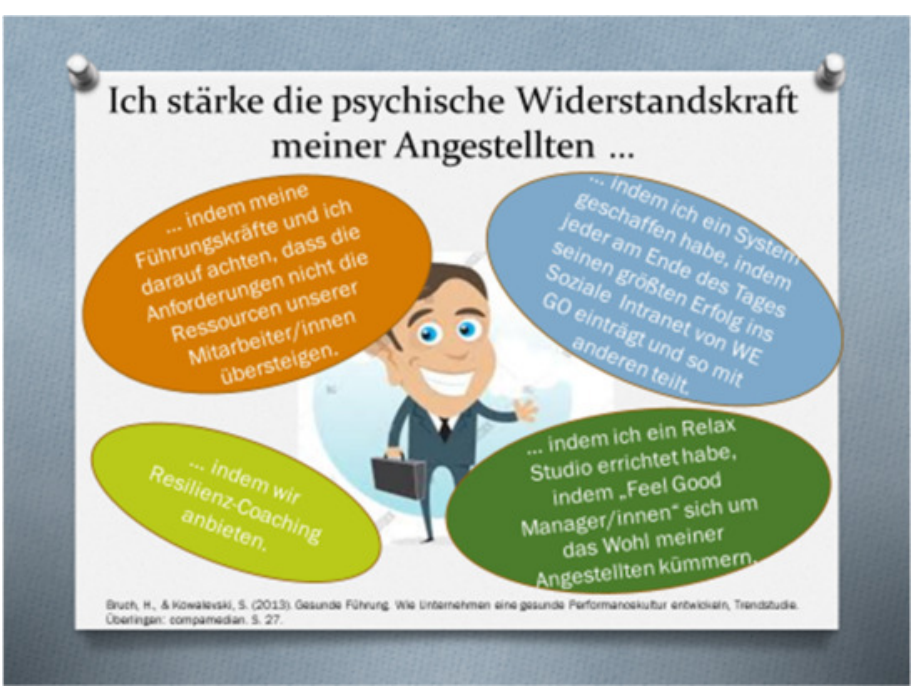

\section{Top Management:}

.....Wir kommen demnächst alle noch mal in einen Wettbewerb um Fachkräfte, gute Fachkräfte. Das ist erst mal ein Thema, mit dem müssen wir uns auseinandersetzen. So, und aus dem Überangebot kommen wir dann vielleicht in eine Situation und sagen, „Sie haben mich zum Vorstellungsangebot eingeladen, ich habe gar keine Zeit, ich gehe mal ganz woanders hin." Also das ist erst mal die Überschrift, die wahrscheinlich in 20, 30 Jahren relevant sein wird, und dann da wieder, gespiegelt mit der Anspruchshaltung, muss man sicherlich auf bestimmte Sachen achten.

....dass wir dahin kommen, den Menschen am Ende des Tages zu sehen. Da bin ich fest von überzeugt, wir als kleine, mittlere Unternehmen haben ja viel bessere Chancen als Großkonzerne, die wahrscheinlich das anbieten werden, ach die brauchen das auch, die werden es auch brauchen vermutlich. Und nicht effizienter, weil ich glaube, das können wir als Menschen nur noch besser darstellen.

...Kein normaler Mann macht das mit, zum Feel-good-Top manager zu gehen, das macht er eine Weile, und dann kommt die Rebellion à la Trump, und das ist noch viel schlimmer, als wenn wir den gar nicht erst dahin schicken würden.

.... dieses Thema „Darauf achten, dass die Anforderungen nicht die Ressourcen der Mitarbeiter übersteigen. "Ich sage mal: Ja, das ist natürlich eine ganz normale Aufgabe, es ist nur die Frage, wie kann ich das gewährleisten: Indem ich aufmerksam bin, wertschätzend bin, im Jahresgespräch das hinterfrage, wie auch immer.

....ich bin auch überzeugt, dass genau dieses alles Antworten sind, die wir so nicht erleben werden. Am Ende des Tages wollen die Leute das auch nicht, glaube ich jedenfalls nicht. ......weil vielleicht sind wir auch alle noch zu weit davon entfernt und in zehn Jahren würden wir anders darauf antworten...... 
...ich glaube so ein System suggeriert auch viel. Also da vermischt sich ja auch Arbeitszeit und private Welt irgendwo, und ich sage mal, wir stellen ja die Arbeitsplätze zur Verfügung, und das Private ist privat. Klar hat das auch Einfluss und man nimmt auch Rücksicht, wenn Schicksalsschläge oder irgendwas passiert, aber ich glaube so ein Feelgood-Top manager könnte auch was suggerieren, was vielleicht gar nicht da ist.

....Einen ganz tollen Auszug habe ich gesehen: .... „Ich habe den Trump gewählt, weil ich gerne wieder möchte, dass alles wieder so wird, wie es früher mal war." Und das ist eigentlich die Antwort. Die Leute sehnen sich nach früher. da sehnen sich die Leute nach einfachen, klaren Antworten, Strukturen. So, das wieder auf unser Thema jetzt übertragen, glaube ich, ja, die Leute wollen klare Rahmenbedingungen, aber es muss sich auch in so einem Korridor bewegen. Und wenn wir jetzt in dieses Thema ResilienzCoaching gehen oder Feel-good-Top manager, dann sind wir schon in diesem Thema "Gutmenschen-Überbehütung", das wollen die Menschen am Ende des Tages glaube ich nicht.....

\section{Mitarbeiter:}

.....Widerstandscoaching fände ich gut. Also dass man da so ein bisschen motiviert und auch gecoached wird.....

.... wenn man die Entscheidung hätte zwischen zwei Unternehmen und alle Parameter sind sehr ähnlich und ich hätte in dem einen Unternehmen so ein System und in dem anderen überhaupt nicht, dann würde glaube ich jeder sofort in die Firma gehen, die sowas schon anbietet, vielleicht sogar, wenn ein paar Parameter schlechter sind, weil das Wohlbefinden vielen Leuten auch wichtig ist. Also umso größer das Unternehmen, umso öfter gibt es das wahrscheinlich heute schon.

....Was ich gar nicht gut finde ist glaube ich so ein soziales Intranet. Das kann auch glaube ich nach hinten losgehen, dass es die Leute anstochert, "Oh, jetzt muss ich auch mal was posten", und "Ich mache das besser als du“......

...das hat mein Vater in seiner Firma, das sind 12 Mitarbeiter insgesamt, da steht ein Kicker im Pausenraum und da wird jeden Tag eine Runde gekickert. Und da kommen die Leute einfach zusammen, alle Fahrer, die den ganzen Tag eigentlich unterwegs sind, und die treffen sich da und die Chefs kommen auch dazu und kickern auch eine Runde mit.

.... ich kenne das auch von dem Kunden, den ich dann besuchen darf, und das ist halt ein großer Konzern und da gibt es dann halt auch Squash-Räume, oder auch im kleineren Rahmen, da steht dann halt eine Tischtennisplatte im Eingangsbereich und da sieht man die da in der Pause dann da man eben eine Runde Tischtennis spielen. Ich denke schon, dass das so ein bisschen die ganze Situation auflockert.

...Alleine, wenn man da mal kurz abschalten will, einfach mal ganz was anderes macht, braucht man ja manchmal. 
10. Slide

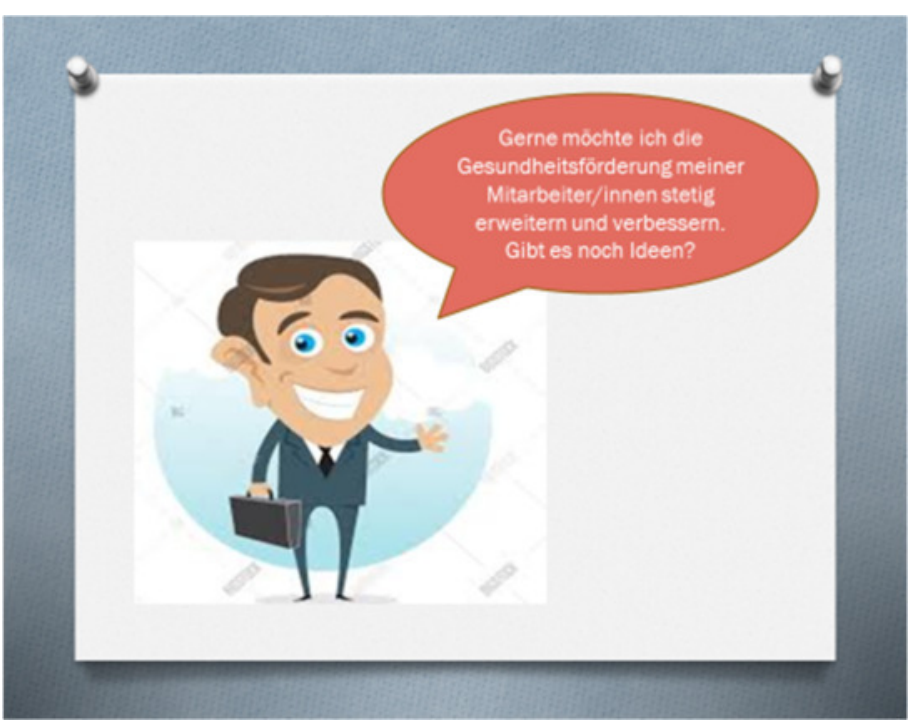

\section{Top Management:}

....Was sicherlich immer schwierig ist, ist Mitarbeitern von oben herunter, das haben wir schon gehabt, so "Chef schlägt vor" oder "Chef schreibt gar vor: Mach mal das und das", oder "Lasst uns mal das und das machen."

.....Gemeinsame Veranstaltungen zu machen. Ich bin eigentlich kein Freund davon, Arbeit und Privates zu vermischen. Was wir aber festgestellt haben ist, dass wir bessere Stimmung im Unternehmen haben, wenn wir regelmäßig gemeinsam was tun..... Die Ideen sind alle da, aber das ist eine gemeinsame Unternehmung, die Richtung Gesundheitsdenken geht oder zumindest eine Empfindung stärkt, die es bei vielen dieser Punkte würde es von oben herab, glaube ich, noch gar nicht so gibt, also von oben herab? Die Leute erreiche ich besser damit, glaube ich.

\section{Mitarbeiter:}

....ich würde sagen mit dem Fördern der Kompetenzen ist eines der wichtigsten Punkte, weil ohne Kompetenzen kann man nichts erreichen.

.....Wertschätzung und Förderung, also wäre mir dann schon wichtig.

.... die Ideen und die Punkte, die der Herr Müller umgesetzt hat, waren alle gut, es muss einfach passen zum Unternehmen, also es muss eine individuelle Lösung sein, die auf das Unternehmen und auf die Teams auch im Unternehmen irgendwie zugeschnitten ist, dass auch jeder daran teilnehmen kann, und dass es vielleicht Maßnahmen gibt, die jeder machen kann, wo auch jeder was von hat, dass erst mal gemeinsam alle was davon haben, um dann vielleicht auch noch mal zu gucken, welche Abteilung, welche Arbeitsgruppen kann ich dann wieder bilden, die dann gezielte Förderung für irgendwas kriegen. Man muss gucken, ....... dass man es nutzen kann, Spaß hat, das irgendwie zu nutzen.... wenn der Vorgesetzte, also am besten der direkte Vorgesetzte das vorlebt, umso einfacher.... 


\section{Slide}

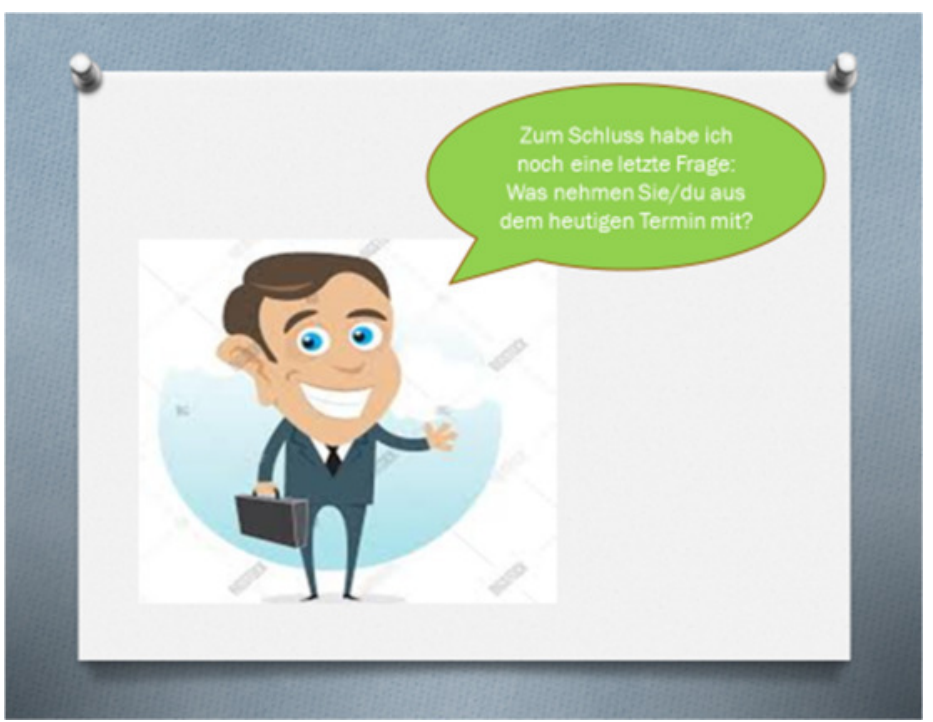

\section{Top Management:}

....Du (Julia) trägst einmal vor, was du da erforscht hast und dann Diskussion. Weil dann die Leute sich damit beschäftigen müssen geistig. Dieses Gesundheitsmanagement ist ja noch nicht in allen Köpfen drin, in meinem war es bis vor kurzem auch noch nicht so richtig drin.

das wäre mal ein super Thema (Ergebnisse Studie von Julia) so bei uns für so einen Führungskreis, wo wir sagen - um dann mal in die gleiche Diskussion zu kommen, weil das glaube ich eine viel größere Lernkurve ist, als wenn wir sagen, „Wir haben jetzt was erfunden.

Was ich auch toll fand, waren auch wirklich so die ganz unterschiedlichen Aspekte, wo man sich ja - erst mal denkt man ja, Gesundheitsmanagement, ach, es ist die Ernährung, das Fitnessstudio, der Gutschein, aber da kamen ja jetzt doch ganz viele Sachen noch hinzu, wo ich auch sagen muss, da habe ich mir noch nie so viele Gedanken darüber gemacht, dass die auf die Gesundheit natürlich auch Einfluss haben - also von daher war es sehr weit gestreut, und sehr, sehr gut.

\section{Mitarbeiter:}

... ich denke mal, dass viele Sachen davon richtig Sinn machen würden, aber viele lassen sich also bei uns jetzt auch nicht wirklich umsetzen.

....nur was das manchmal fehlt, das sind die Ressourcen finanzieller Art oder auch personeller Art, um dies vernünftig über die Runden zu bringen. Alles das, was der Herr Müller vorhat, kostet ja auch alles richtig Geld. So, und ob dieses Geld dann immer zur Verfügung gestellt wird? Ein Chef möchte das ja auch immer gerne gerechnet haben....und der will am Ende sehen, da kommt was bei raus. Und das muss man erst mal nachvollziehen können. 
....es muss ja immer erst was Negatives passieren, dass sich das Denken auch ändert, finde ich. Ja, da muss man guter Hoffnung bleiben, dass es irgendwann einfach mehr auf den Menschen ankommt als auf den Umsatz oder die Zahlen, dass halt die Waagschale so ein bisschen anders ist. .....Wie gesagt, es muss erst mal das Umdenken kommen, finde ich und dann sind vielleicht auch Chefs bereit ein bisschen mehr Budget dafür zu geben als es jetzt halt ist.

....im Kleinen oder im Mittelstand das umzusetzen, ist wahrscheinlich das Schwierigste Budget lockerzumachen und einzusehen, dass ich am Ende was davon habe, was ich halt nicht in Zahlen messen kann wirklich.

....einiges können wir mitnehmen, ....nun müssen wir gucken, was wir umsetzen können, mit der Wertschätzung des Mitarbeiters und so, und Einzelgespräche vielleicht mal führen und das intensiver auch noch.

.... Für mich ist es eine Traumvorstellung, dass es so ablaufen könnte in einem Unternehmen, und dass ich mir wünschen würde, dass es so eigentlich überall umgesetzt wird.

....Man hört immer mehr von solchen Szenarien in Unternehmen, und ja, es wäre schön, wenn das im Mittelstand irgendwann ankommen würde. 